UNITED STATES DEPARTMENT OF THE INTERIOR

GEOLOGICAL SURVEY

\title{
Geochemical analyses and summaries of shale from Kentucky
}

By

Jon J. Connor

Open-File Report 81-1097

This report is preliminary and has not been edited or reviewed for conformity with U.S. Geological Survey standards or nomenclature 
Introduction. $\ldots \ldots \ldots \ldots \ldots \ldots \ldots \ldots \ldots \ldots \ldots \ldots \ldots \ldots \ldots \ldots \ldots \ldots \ldots \ldots$

Stratigraphic setting...............................

Analytical methods.................................... 4

Sampling design..................................... 4

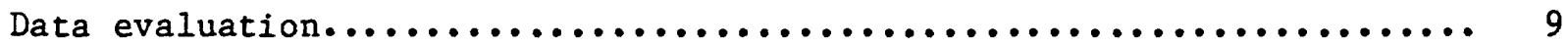

Mineralogy........................................... 10

Geochemical variability............................... 13

References cited.................................... 47

\section{Illustrations}

Figure 1. Regional stratigraphic relations of Paleozoic rocks in

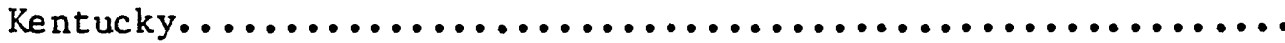

2. Location of $7-1 / 2^{\prime}$ quadrangles from which shale rocks of Paleozoic age were collected in Kentucky.............. 6

3. Comparison of theoretical, planned, and actual distances between samples and between sampling localities.............

4. Average distance (error) between planned and actual sampling sites........................................ 8

5. Plot of normative mineralogy in Paleozoic shale in Kentucky.. 11

6. Geochemical frequency distributions in Paleozoic shale from Kentucky.................................... 16 
Table 1. Chemical analyses of Paleozoic shale from Kentucky:

A. Chattanooga, New Albany, and Ohio shales.............. 49

B. Shale of Lower Mississippian age.................. 57

C. Shale of Upper Mississippian age................... 63

D. Shale of Pennsylvanian age...................... 72

2. Elements commonly looked for, but rarely or never detected, by direct-reader emission spectrographic analysis.......... 84

3. Average modes for Paleozoic shale from Kentucky............ 85

4. Sampling sites for Paleozoic shale from Kentucky:

A. Chattanooga, New Albany, and Ohio Shales.............. 86

B. Shale of Lower Mississippian age................... 89

C. Shale of Upper Mississippian age.................. 92

D. Shale of Pennsylvanian age....................... 96

5. Components of geochemical variance for Paleozoic shale from Kentucky:

A. Chattanooga, New Albany, and Ohio Shales............... 102

B. Shale of Lower Mississippian age................... 103

C. Shale of Upper Mississippian age................... 104

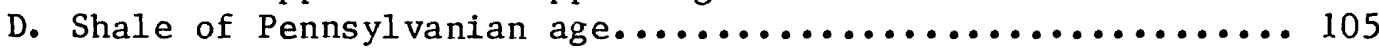

6. Summary geochemical statistics for Paleozoic shale from Kentucky:

A. Chattanooga, New Albany, and Ohio Shales.............. 106

B. Shale of Lower Mississippian age................... 108

C. Shale of Upper Mississippian age................... 110

D. Shale of Pennsylvanian age....................... 112 
This report summarizes results of a study of geochemical variability in shale of Paleozoic age in Kentucky. The study was undertaken as a field experiment in geochernical sampling and was based on an attempt to collect samples of shale from outcrop in an objective fashion. Kentucky is typical of many areas in the cratonic part of the eastern United States in that a combination of low relief and pervasive weathering has resulted in a general paucity of bedrock exposures. This study illustrates a few of the effects of such constraints on objective sampling. These difficulties notwithstanding, the data resulting from this study were used to identify 6 stratigraphic-geochemical subpopulations in the Paleozoic shale of Kentucky. An earlier report (Connor, 1981) described the results of a similar study of carbonate rocks.

Kentucky was chosen for these studies because of an ongoing U.S. Geological Survey-Kentucky Geological Survey cooperative mapping program at the time sampling was undertaken (1964-1965). Although only a third or so of the State was covered by mapping at that time, the available 7-l/2'-scale maps formed a reasonably solid stratigraphic base for relating the samples on a regional scale. Because a prime requirement for geochemical target definition is that it be unequivocally identifiable in the field, sampling was restricted to those areas covered by $7-1 / 2^{\prime}$ quadrangle naps at the time of sample selection.

Numerous U.S. Geological Survey mappers contributed time and effort in helping to locate outcrops for sampling. L. Artis, S. Botts, G. Chloe, Nancy Conklin, Paul Elmore, J. Glenn, R. G. Havens, W. W. James, Lorraine Lee, L. F. Rader, H. Smith, D. Taylor, and J. A. Thomas analyzed over 200 rock samples for some 50 elements for this work. Mel Johnson made thin sections of each sample.

\section{STRATIGRAPHIC SETTING}

Shale of Ordovician, Silurian, Devonian, liississippian, and Pennsylvanian age crop out in Kentucky (fig. l). No shale of Ordovician or Silurian age was sampled in this study because of limited outcrop (Siliurian) or paucity of mapping (Ordovician) at the time of collection. The most lithically distinct shale in the Paleozoic of Kentucky is the organic-rich deposits of the Chat tanooga, New Albany, and Ohio Shales (Upper Devonian to Lower Mississippian). This unit is generally less than $15 \mathrm{~m}$ thick over much of the State. The strata thicken northward to a maximum of $70 \mathrm{~m}$ in northeastern Kentucky, where the Ohio Shale is overlain by deltaic deposits of the Berea and Bedford Formations. These two formations are, in turn, overlain by another organic-rich shale--the Sunbury Shale. Conant and Swanson (196l, p. 50) concluded that these black shales were deposited on a stable interior platform ringed by areas of low relief. One sample in this work (DSH-Plll, in the Briensburg Quadrangle of western Kentucky) was white and contained less than $1 \%$ organic carbon. Cluff and Reinbold (1978) reported that similar interbeds in the Illinois Basin reflected deposition in an oxygenated interval of an oxygen-stratified basin. 


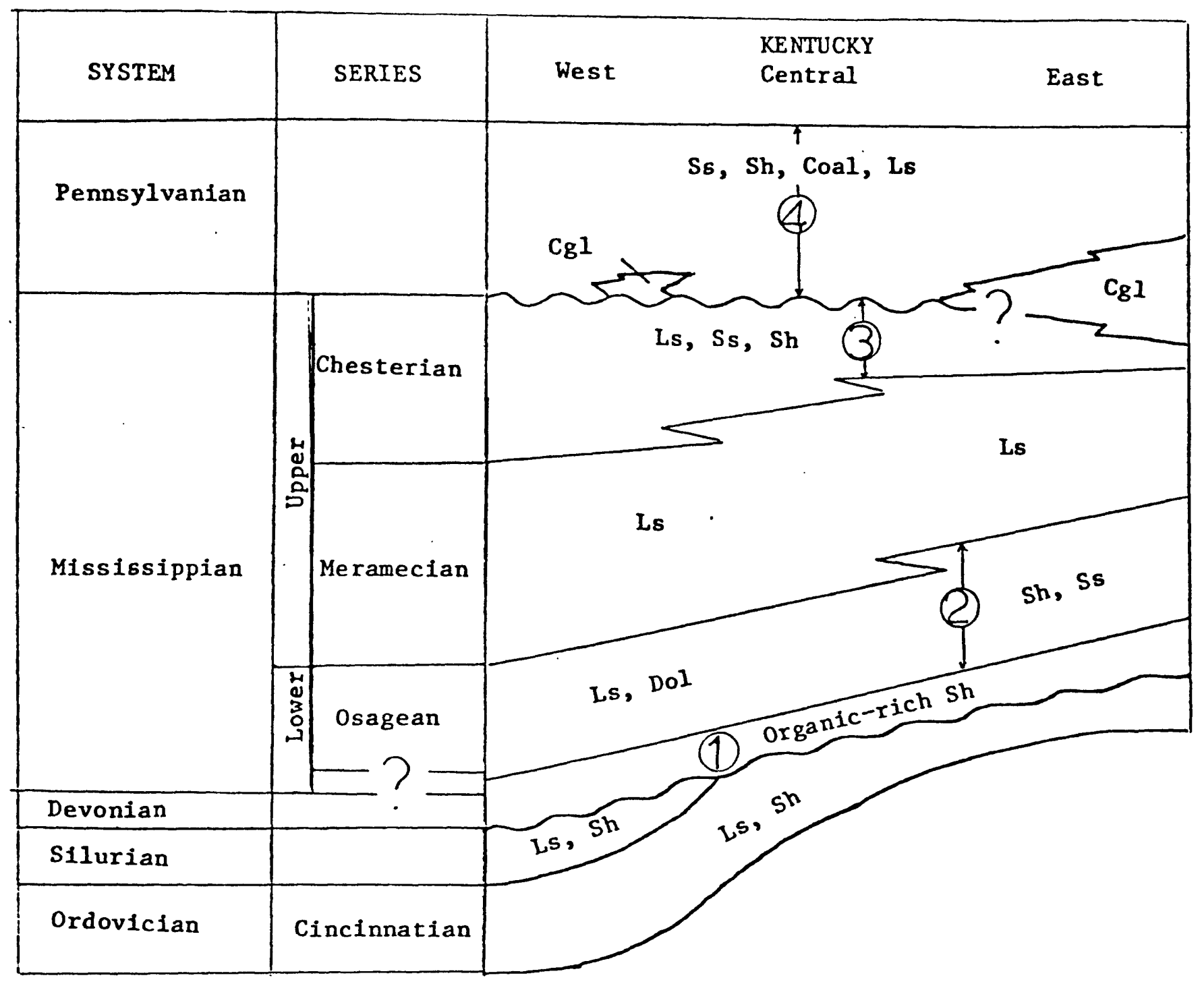

Figure 1.--Regional stratigraphic relations of Paleozoic rocks in Kentucky. Sampled shale units are numbered. 
Shale of Osagean age (Lower Mississippian) in Kentucky is represented largely by the Borden and Fort Payne Formations (Sable, 1979). These formations consist of a heterogeneous assortment of interbedded limestone, dolomite, sandstone, shale, siltstone and chert. Deltaic clastics are dominant to the northeast (Borden Foruation) whereas carbonates are dominant to the southwest (Fort Payne Formation). In northeastern Kentucky, the Borden attains a thickness of more than $200 \mathrm{~m}$ and consists of shale, siltstone, and fine-grained silty sandstone (Chaplin and Mason, 1978). In western Kentucky the Fort Payne ranges up to $200 \mathrm{~m}$ in thickness (Lambert and MacCary, 1964), and consists of dark, finely crystalline, cherty limestone or dolomitic limestone. In west-central and south-central Kentucky, the Borden-Fort Payne interval contains interbedded shale, sandstone and crinoidal limestone (Thaden and others, 1961, p. B88; Kepferle, 1966). Bedded chert is locally prominent in this same area in outcrop. The uppermost Borden strata of east-central Kentucky are apparent1y time equivalents of the lowermost strata in the Salem-Warsaw interval of west-central Kentucky (Sable and others, 1966).

The Borden-Fort Payne interval is overlain by a highly variable thickness of nearly pure limestone (mostly of Meramecian age), which in turn is overlain by an interbedded sequence of limestone, sandstone and shale (mostly Chesterian in age). Together, these rocks, which comprise nearly all of the Upper Mississippian Series in the State (Sable, 1979), exceed $600 \mathrm{~m}$ in thickness in western Kentucky where 18 formations are recognized. These strata thin to about $200 \mathrm{~m}$ over the Cincinnati Arch in south-central kentucky and are locally absent in northeastern Kentucky because of pre-Pennsylvanian erosion.

The Meranecian strata mark the peak of Mississippian transgression in Kentucky and consist of light- to dark-gray, finely to coarsely crystalline, cherty, fossiliferous limestone. The Chesterian rocks reflect the closing phases of Mississippian deposition in western Kentucky. In the Illinois Basin, this phase consisted of at least 70 reversals of shore-line movement (Swann, 1964, p. 654) before final withdrawal of the sea to the southwest (Siever, 1951, p. 575). In eastern Kentucky, the Chester is represented by the Pennington Formation, a sequence of interbedded limestone, sandstone and shale. Near the Tennessee line, the Chester includes the Hartsel and Bangor Formations.

The Pennsylvanian rocks comprise a heterogeneous assortment of interbedded, poorly sorted sandstone, shale, siltstone, claystone, coal, conglomerate and carbonate. These rocks are more than $900 \mathrm{~m}$ thick in the Illinois Basin and over $1500 \mathrm{~m}$ thick in the Appalachian Basin of southeastern Kentucky (McKee and Crosby, 1975, P1. 11). In many outcrops, the base of these rocks is marked by a conspicuous unconformity separating interlayered, well bedded, fine-grained carbonate and clastics below from interfingering conglomeratic sandstones and carbonaceous siltstone or shale above. In eastern Kentucky, the relation of the basal conglomerate in the Lee Formation to the underlying shale of the Mississippian Pennington Formation is in dispute. Wanless (1975a, p. 23) notes the presence of intertongues of Lee-type rocks in the Pennington in both northeastern and southeastern Kentucky, and Horne, Ferm and Swinchatt (1974) suggest that this contact may not even be disconformable.

The great bulk of the Pennsylvanian interval, however, contains no conglomerate but rather represents rapid accumulation of sandstone and shale with interbeds of coal and carbonaceous shale. These deposits are particularly 
thick in extreme southeastern Kentucky reflecting proximity to source (Wanless, 1975a). The deposits were laid down on a large piedmont alluvial fan built westward or northwestward from the Appalachian geosyncline (Siever, 1951, p. 578). Sources of the Pennsylvanian rocks of western Kentucky apparently lay to the northeast (Wanless, 1975b).

\section{ANALYT ICAL METHODS}

Each sample was trimmed of obvious weathering rinds, where possible, and about $200 \mathrm{~g}$ were crushed, ground to pass 100 mesh and split into two parts. Duplicates from each season's collection were placed in a randomized sequence prior to submission to the laboratory in order to circumvent analytical drift. All chemical analyses were performed in laboratories of the U.S. Geological Survey. The common rock-forming oxides were determined by rapid rock methods in Washington, D. C., as described in Shapiro and Brannock (1962). Most trace elements were determined in Denver, Colo., by a direct-reading emission spectrographic method described in Havens and Myers (1973). Mercury and silver in the organic-rich shales were determined by atomic absorption. Total carbon in these shales was determined by induction furnace combustion, carbonate carbon was determined gasometrically, and organic carbon was determined by difference.

The geochemical analyses are listed in tables 1A-1D. All samples (except LMSHE 211, LMSHE 212, LMSHE 221 and LMSHE222 in table 1 , and UMSHK 111 and UMSHK 112 in table 1C) were analyzed in duplicate. Thus in table $1 \mathrm{~A}$, the first two rows (DSH-E111) are replicate analyses of a single sample; the next two rows (DSH-E112) are replicate analyses of another sample; and so forth. Trace elements commonly looked for but rarely detected by emission spectrography are listed in table 2 along with their approximate lower limits of determination. Thin-section modes, based on 50 points per section, were counted on all samples. Average modes are listed in table 3.

\section{SAMPLING DESIGN}

Four Paleozoic shale units were sampled in this work (fig. 1); in ascending stratigraphic order, they are

1. Organic-rich shale of the Chattanooga, New Albany, and Ohio Shales (Upper Devonian and Lower Mississippian)

2. Shale of Lower Mississippian age (Borden and Fort Payne Formations)

3. Shale of Upper Mississippian age (probably all of Chesterian age)

4. Shale of Pennsylvanian age

The same sampling design was used for each stratigraphic unit. The design is a hierarchical one in which each level of the design includes paired sampling units separated by a given distance or a range of distances. The State was arbitrarily divided into one-degree areas. For each stratigraphic unit sampled, two mapped 7-1/2' quadrangles were selected randomly within each one-degree area, if possible. (For some one-degree areas and some stratigraphic units, only two quadrangles were available). In each $7-1 / 2^{\prime}$ quadrangle two sampling localities of a size 300 by $450 \mathrm{~m}$ were selected randomly from those parts of the quadrangle underlain by the unit. In each locality, two random samples of $4-8 \mathrm{~kg}$ were taken from outcrop. For the organic-rich shale (unit 1 ) and the Borden-Fort Payne shale (unit 2), two random samples were collected from an 
exposed stratigraphic section in each locality. (Units 3 and 4 were too thick to permit exposure of a complete section within a locality.) The quadrangles are listed in figure 2, and the sampling sites are described in tables 4A-4D. The quadrangle pairs in each one-degree area for each stratigraphic unit are identified in Part II of tables 6A-6D. Because of lack of suitable outcrop, one pair of 7-1/2' quadrangles for the organic-rich shale came from different one-degree areas (Briensburg and Eddyville quadrangles), as did a pair from the Upper Mississippian unit (Wrigley and Portsmouth quadrangles). For a similar reason, in one area of Borden rocks, three adjacent quadrangles (Head of Grassy, Wesleyville and Brushart) were used instead of two.

Histograms of the theoretical, planned and actual distributions of distance between samples in each locality and between localities in each 7-1/2' quadrangle are shown in figure 3A. The theoretical frequency distribution of a distance between two points in a rectangle was based on work of Ghosh (1951). Figure $3 \mathrm{~B}$ shows the distribution of samples through stratigraphic sections of units 1 (organic-rich shale) and 2 (Borden and Fort Payne Formations). In figure $3 \mathrm{~A}$, the planned frequency distributions are based on distances between sample pairs (or locality pairs) as picked in the office using randomization procedures. The actual frequency distributions are based on distances between sample (locality) pairs as actually occurred in the field. The planned distances are slightly less than the theoretical distances; the actual distributions of distance between samples in a locality, however, are strongly biased with respect to both the planned and theoretical distances. This bias, which results largely because of outcrop constraints, indicates the severity with which operational problems can influence sampling design. Theoretical and actual average distances associated with three levels of the design are compared below:

$\begin{array}{lcc}\begin{array}{c}\text { Level of sampling design } \\ \text { and rock unit }\end{array} & \begin{array}{c}\text { Theoretical } \\ \text { distance }(\mathrm{km})\end{array} & \begin{array}{c}\text { Actual } \\ \text { distance }\end{array} \\ \begin{array}{l}\text { Between samples: } \\ \text { Upper Mississippian }\end{array} & .27 & .091 \\ \text { Pennsylvanian } & .27 & .23 \\ \text { Between localities: } & & \\ \text { Organic-rich shale } & 6.4 & 5.2 \\ \text { Lower Mississippian } & 6.4 & 6.0 \\ \text { Upper Mississippian } & 6.4 & 4.0 \\ \text { Pennsylvanian } & 6.4 & 6.0 \\ \text { Between quadrangles: } & & \\ \text { Organic-rich shale } & 53 & 24 \\ \text { Lower Mississippian } & 53 & 30 \\ \text { Upper Mississippian } & 53 & 31 \\ \text { Pennsylvanian } & 53 & 33\end{array}$

Commonly, a randomly picked locality lacked outcrop. In such a case, samples were collected as close as possible to the randomly picked locality. The errors resulting from such problems in locating samples are diagrammed in figure 4. 


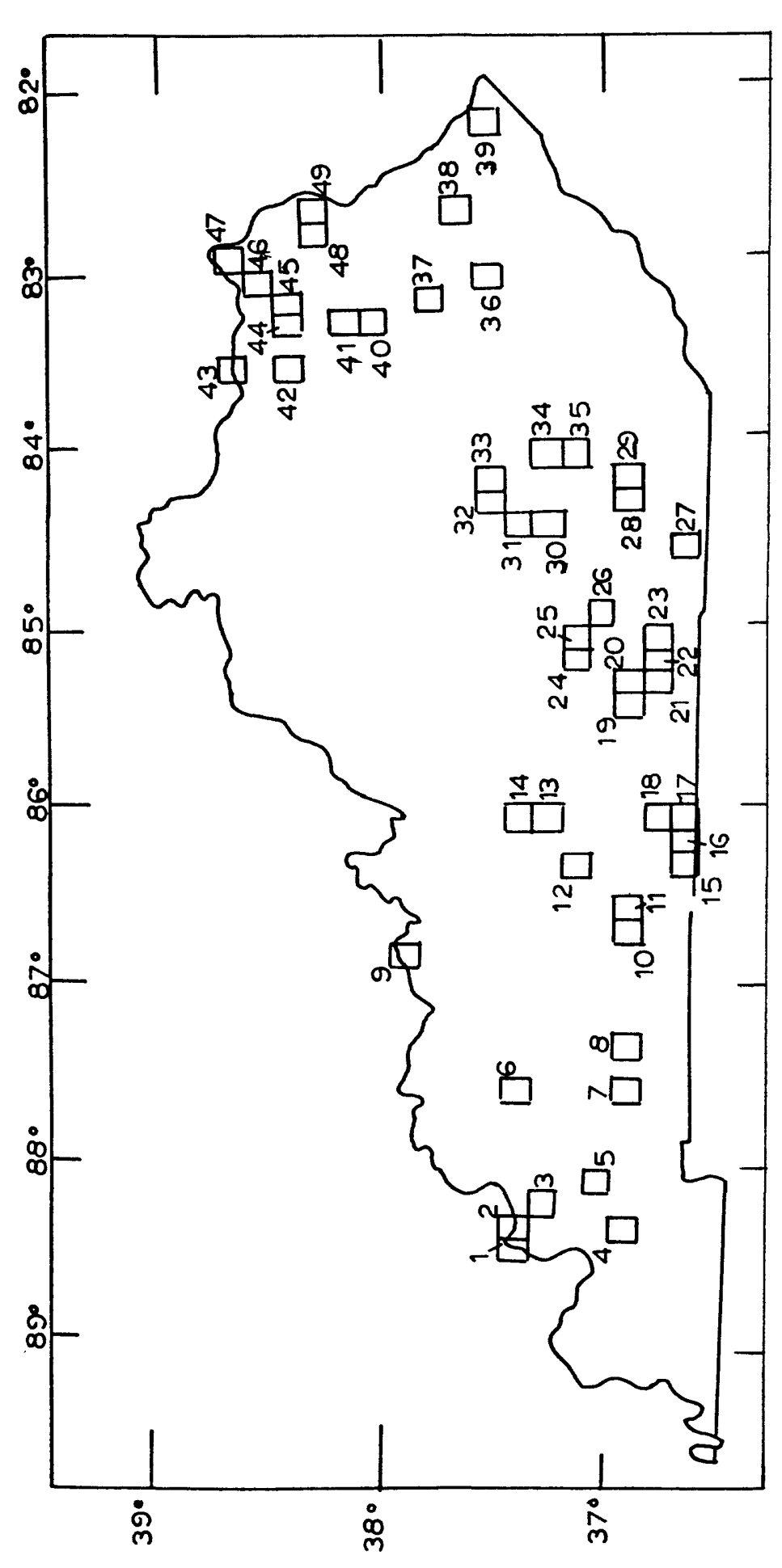

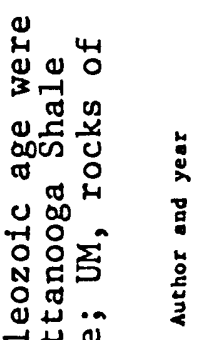

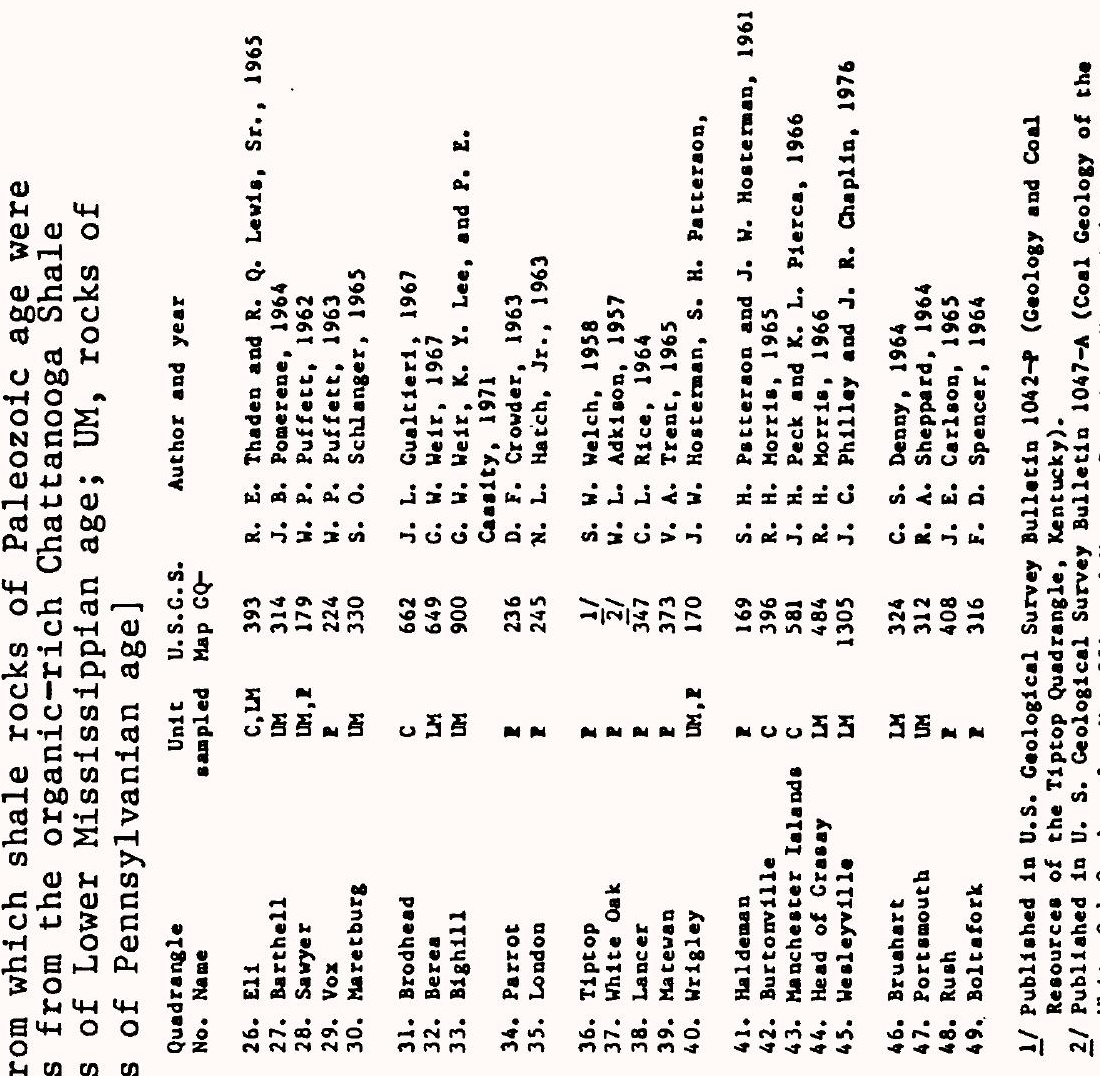
की

U

ब ${ }_{\pi}$ का

$\rightarrow \infty$ on

㫣䗆

का 出

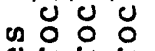

ه广⿻ 丨क

禹

㫣纪

तु.

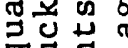

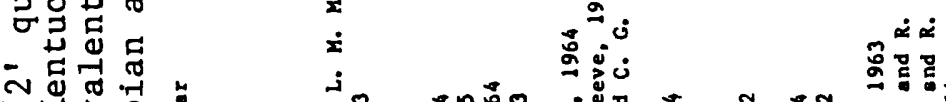

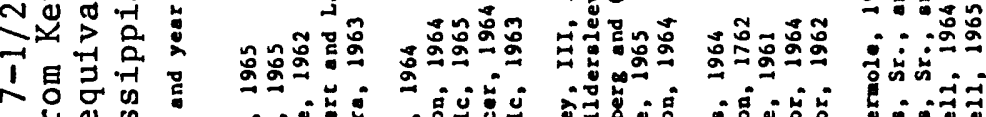

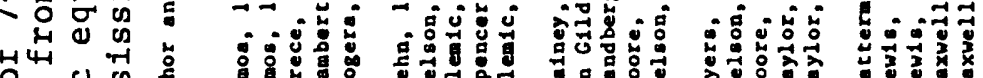

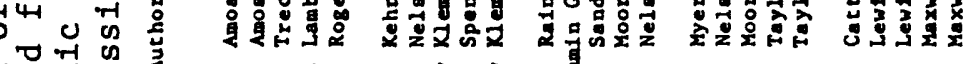

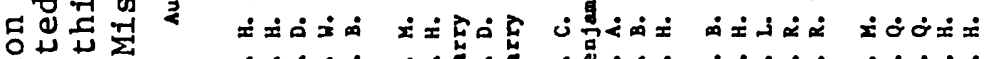

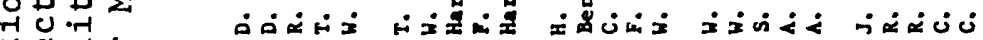

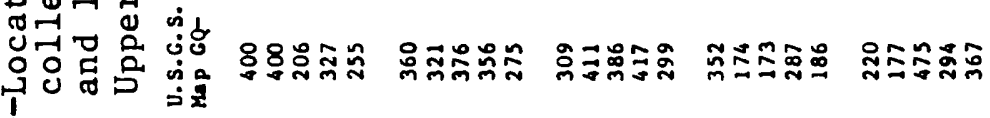

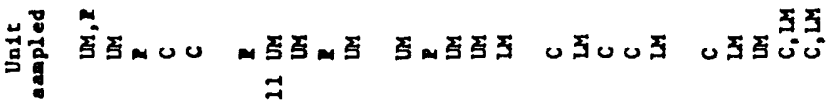

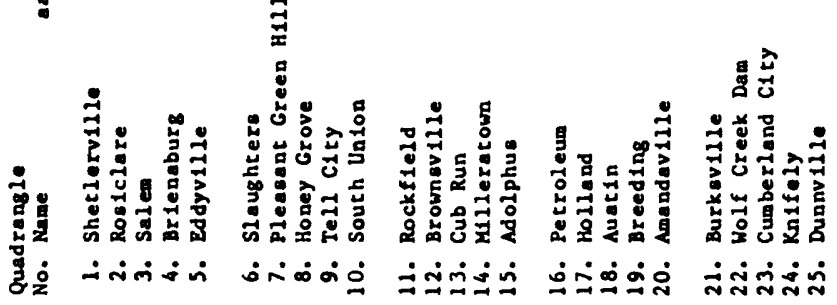



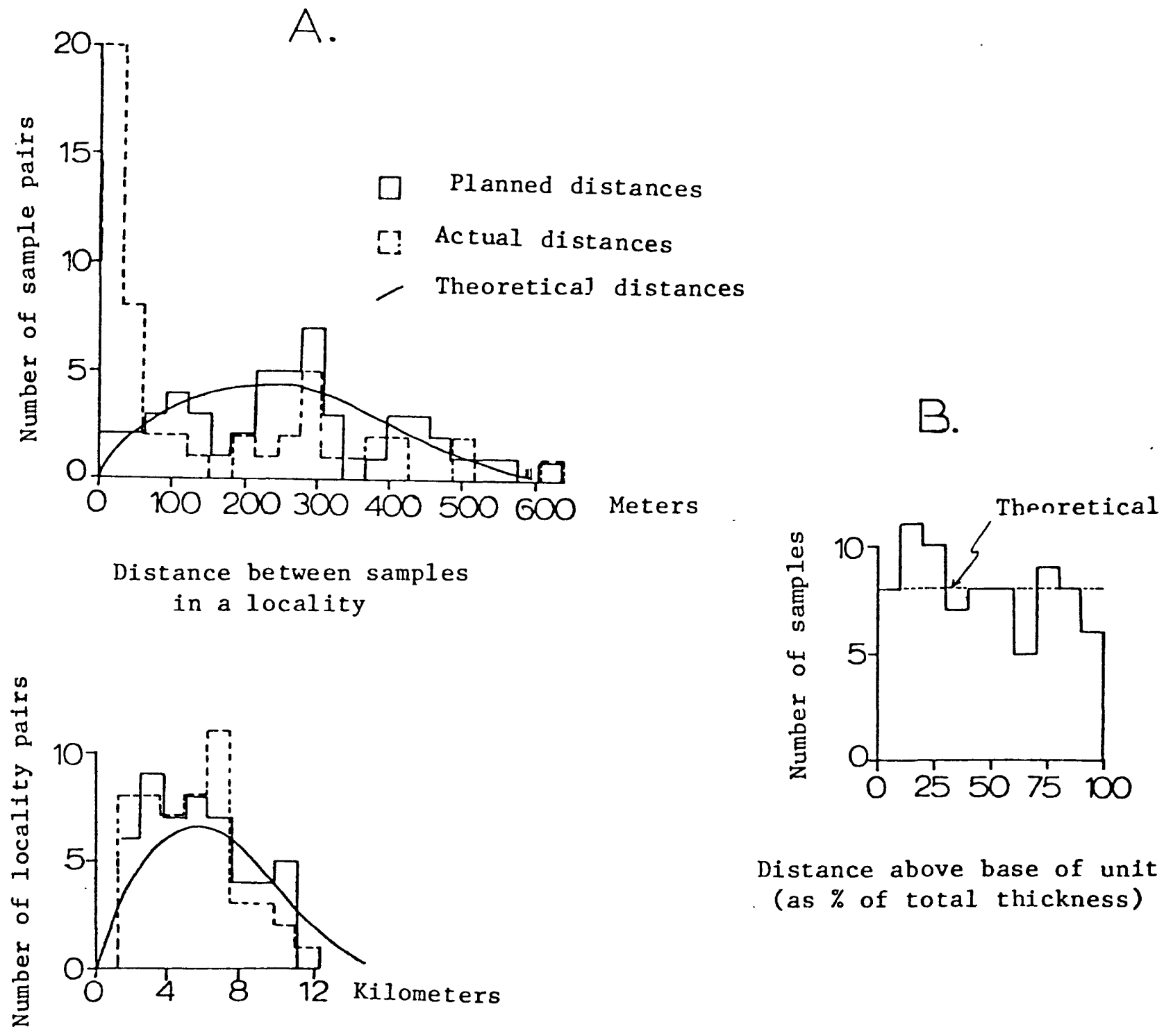

Distance between localities in a 7-1/2' quadrangle

Figure 3.--A. Comparison of theoretical, planned and actual distances between samples and between sampling localities in the hierachical sampling plan used in the study of the Upper Mississippian and Pennsylvanian shale units in Kentucky.

B. Distance above base of unit for samples from the Chattanooga (and equivalents) and Lower Mississippian shale units in Kentucky. Distance above base measured as a percentage of total thickness of each unit. 

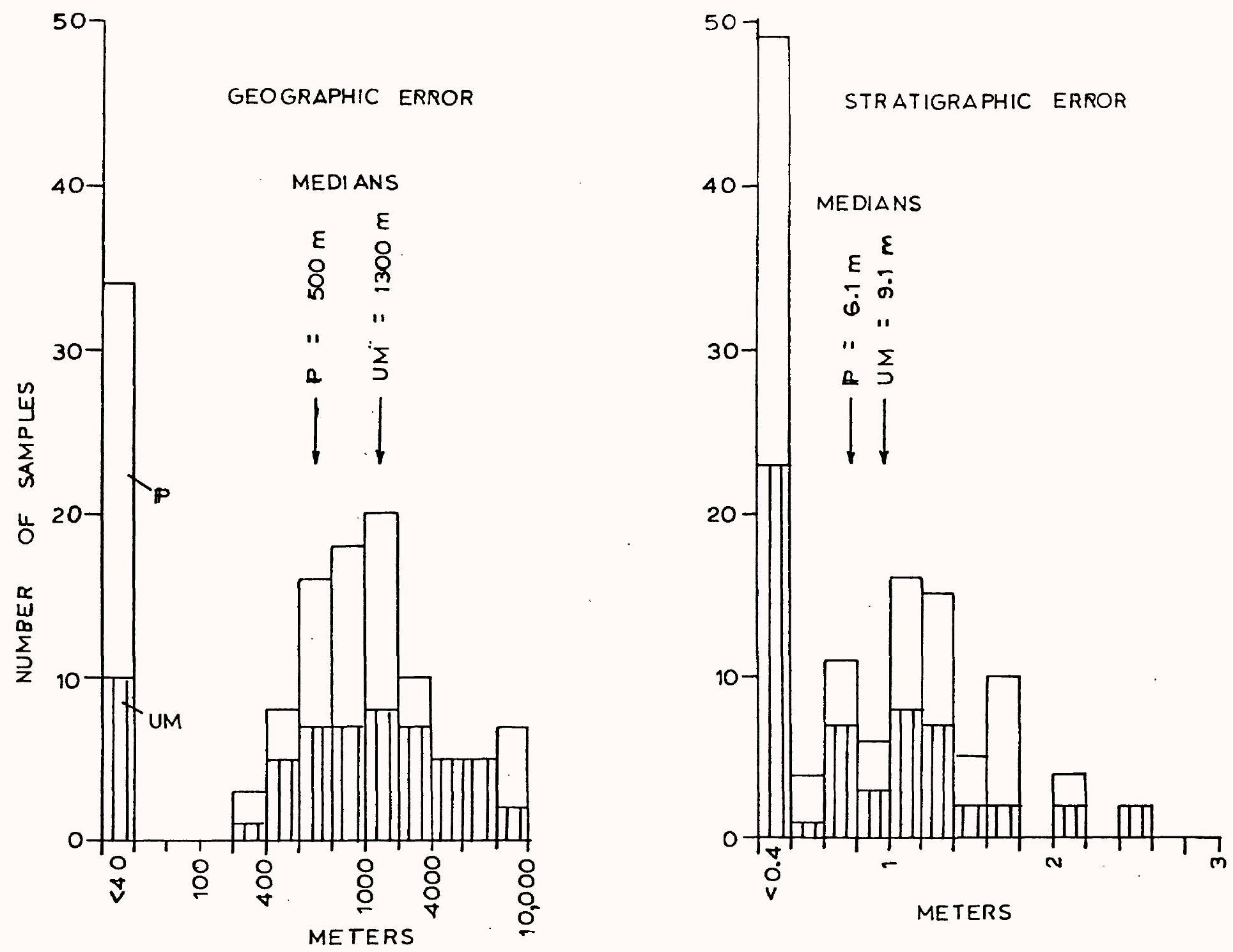

Figure 4.-
- Average distance (error) between planned and actual
sampling sites for samples of Upper Mississippian [UM]
and Pennsylvanian (P) shale in Kentucky. 
The sampling design is a special case of the analysis of variance. The statistical model is

$$
\log X(i j k l m)=M+A(i)+B(i j)+C(i j k)+D(i j k 1)+E(i j k l m)
$$

where $X(i j k l m)$ represents an analytical value reported by the analyst; $M$ represents the grand average (in logs) for that element in the stratigraphic unit (or sampling target) and the remaining terms are deviations, each reflecting a separate source of variation. The first term, A(i), represents the difference between $N$ and the average (in $\log s$ ) for the ith sampled one-degree area; the second, $B(i j)$, represents the difference between the average (in logs) of the jth 7-1/2' quadrangle and the ith one-degree area; the third, $C$ ( $i j k)$, represents the difference between the average (in $\operatorname{logs}$ ) of the $k t h$ sampling locality and the jth 7-1/2' quadrangle; the fourth, $D(i j k 1)$, represents the difference between the average. (in logs) of the 1 th sample and the kth locality, and the fifth, $E(i j k l \mathrm{~m})$, represents the difference (in logs) between the observed analytical result and the true (but unknown) concentration. Logarithms of concentration in trace element work are commonly employed to help meet some of the assumptions underlying the statistical procedures used in data analysis (Miesch, 1976, p. 7-12). The estimate of total logarithmic variance in each sampled unit is equal to the sum of the estimates of the five components defined in equation ( 1 ):

$$
V(\log X)=V(A)+V(B)+V(C)+V(D)+V(E)
$$

where $V(\log X)$ is the total logarithmic variance.

\section{DATA EVALUATION}

Summary statistics of most of the distributions in this study are based on logarithms of the data. Thus, average values are given either as geometric means (GM) or as medians (M). In general, medians were calculated for sample subsets containing less than 10 samples (or 20 analyses where each sample was analyzed twice), and geometric means were calculated for sample sets of 10 or more. The scatter about these averages is measured by the geometric deviation (GD), a factor useful in computing expected ranges in concentration. For example, if a distribution is lognormal, about 68 percent of the determinations in a randomly selected suite should fall within the limits GM/GD to GMxGD, and about 95 percent should fall within the range G1/GDS to GMxGDS, where GDS is the square of $\mathrm{GD}$.

A geochemical constituent is censored if a sample suite contains one or more samples in which the concentration is too low to be measured by the analytical method used. Where a constituent was censored, the mean and variance of the distribution, or their logarithmic counterparts, were adjusted in an unbiased manner (Miesch, 1976, 41-46). The analysis of variance, however, requires completely uncensored data, and the following arbitrary practice was used to circumvent problems of censoring. If a third or less of the frequency distribution was censored, a value equal to approximately seven-tenths of the lower limit of determination was used in place of the censored values. (If more than a third of the distribution was censored, the constituent was not subjected to the analysis of variance.) The justification for such a replacement is that substitution of any reasonable value below the analytical limit would not 
substantially alter geochemical conclusions drawn from the statistical analysis. The analysis of variance results are given in tables $5 \mathrm{~A}-5 \mathrm{D}$.

Where the analysis of variance indicated significant geochemical differences among areas (most areas in this work being represented by a pair of sampled 7-1/2' quadrangles), median values are listed for the areas (Part II, tables 6A-6D). A basic criterion for the sufficiency of differences among these medians is the conventional F-statistic, which is based on measures of variance between and within areas. If the F-statistic is found to be significantly different from zero, one can have a prescribed confidence that, at the least, one of the areas is different from some other.

A more stringent criterion, described by Miesch (1976, p. 101), requires that the mean variance ratio, $v(m)$, exceed a value of 1.0 if differences among area means are to be viewed as reproducible. This ratio for balanced designs based on equation ( 1 ) is defined as

$$
\mathrm{v}(\mathrm{m})=\mathrm{V}(\mathrm{A}) / \mathrm{D}
$$

where $\mathrm{D}$ is defined as

$$
D=V(B) / 2+V(C) / 4+V(D) / 8+V(E) / 16
$$

The denominators in equation (4) are products of the number of sampling units defined at each level of the hierarchical design, which in this work was set to 2.0. Non-response in sampling and recognition of geochemical subpopulations following collection, however, resulted in some of the sampling units being less than 2.0. Where this happened, the resulting bias in $v(m)$ is footnoted (tables $5 C, 5 \mathrm{D})$.

\section{MINERALOGY}

The normative mineralogy of the samples used in this study is shown in figure 5. The organic-rich shale (Chattanooga and lithic equivalents) was plotted on a quartz, illite, organic C triangle. Organic $C$ is less abundant in the other shales. Although many Pennsylvanian shales are gray, they rarely contained more than a percent organic C. All Al203 was assigned to illite of 26 percent AL203 composition, and excess SiO2 assigned to quartz. For normative calcite and dolomite, concentrations of $\mathrm{CaO}$ and $\mathrm{MgO}$ were reduced slightly prior to computation in order to account for minor $\mathrm{CaO}$ and $\mathrm{MgO}$ in clay. Initially, all $\mathrm{MgO}$ was assigned to dolomite and excess CaO assigned to calcite. If these two minerals summed to less than 40 percent (which was common), the original MgO and $\mathrm{CaO}$ concentrations were reduced by one percent and one-half percent, respectively. If, in addition, the ratio of normative illite to normative quartz exceeded 2.0 (also common), the original $\mathrm{MgO}$ and $\mathrm{CaO}$ concentrations were reduced by two percent and one percent, respectively, and the two carbonate minerals computed one more time. 


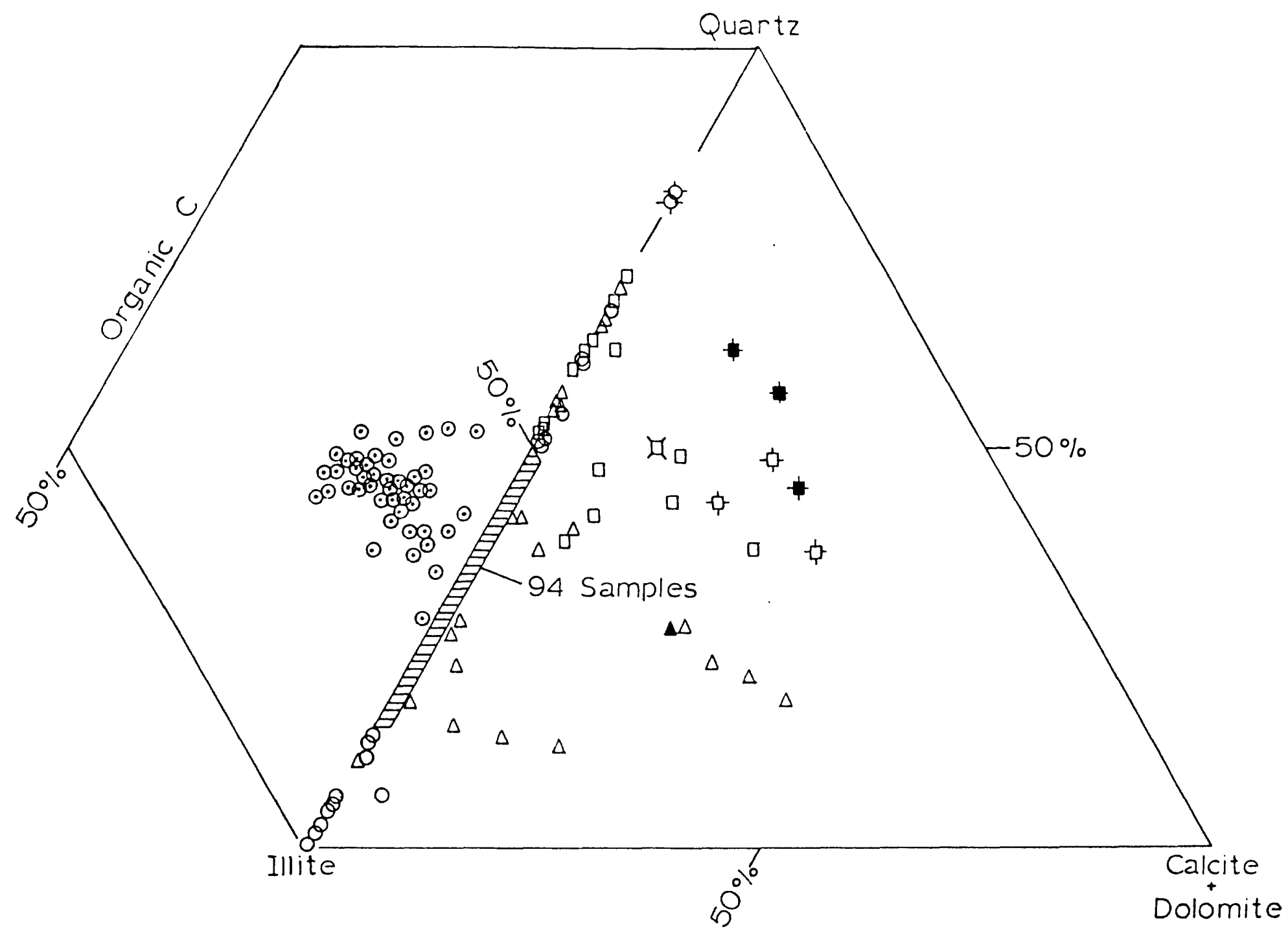

Figure 5.--Plot of normative mineralogy of and organic C in Paleozoic shale in Kentucky.

Stratigraphic unit

Pennsylvanian

Siliceous shale

Upper Mississippian

Lower Mississippian Sandy shale

Modal chert > 25\%

Chat tanooga and lithic equivalents
Shale Dolomite>Calcite

(where total

carbonate $>10 \%$ )
0

$\phi$

$\Delta$

号

$\odot$ 
In thin section, the organic-rich rocks are finely laminated and variably silty. The organic material imparts some of the layering to the rock and, where oxidized, the texture takes on a felty appearance. Quartz, feldspar, flattened rock fragments and Tasmanites are common framework grains. The rock fragments are mostly clay shale or micaceous siltstone although a few appear to be phyllitic. The Tasmanites are translucent orange, isotropic, flattened rings, some of which are broken, convoluted or corrugated. Muscovite is common; secondary dolomite (in rhombs) and gypsum and hematite (after pyrite) are present locally.

Both the silt and the dolomite locally form distinct lamellae. The silt is commonly mixed with fine sand and averages about $0.03 \mathrm{~mm}$ in maximum diameter. Sample DSH-Rlll (from the Petroleum quadrangle) contained a layer of angular sand grains as much as $0.2 \mathrm{~mm}$ in maximum diameter, that consisted of severely strained, polycrystalline quartz, glauconite, clay shale fragments and chert(?). The dolomite rhombs average about $0.1 \mathrm{~mm}$ in diameter and some show overgrowths. Pyrite is locally abundant, both as variably sized spheres (ranging from <.01-. 2 $\mathrm{mm}$ in diameter) and larger ragged or cubical masses. Sample DSH-S212 (from the Breeding quadrangle) contained a cruciform mass of pyrite cored with gypsum(?). The laminations commonly bend around the pyrite indicating early formation (pre-lithification) of the pyrite.

The shales of Lower Mississippian age range from nearly pure claystone to argillaceous siltstone comprised of widely varying amounts of poorly sorted, angular detritus. Many of the claystones were collected from the basal Nancy Member of the Borden Formation. A few samples from south-central Kentucky (Amandaville, Wolf Creek Dam and Knifely quadrangles) were very calcareous and contained sand-sized grains up to $0.1 \mathrm{~mm}$ in diameter. This sandy shale is probably genetically related to the coarse-grained, high-energy deposits of the Fort Payne Formation in this area (for example, the Knifely Sandstone Member of the Fort Payne of Kepferle and Lewis, 1974).

The framework grains are dominated by quartz (or, in the case of the sandy shale, by carbonate clasts), which is generally clear although some stretched and polycrystalline grains are present. Potassium feldspar, plagioclase, clay pellets, glauconite, detrital chert, pelitic (schistose) fragments, muscovite, altered biotite, numerous heavy minerals, opaques (including pyrite in samples LMSHL 121 and LMSHS212 from the Eli and Amandaville quadrangles, respectively), and phosphatic pellets were seen. Iron oxide stained much of the matrix and its intensity may reflect the degree of weathering imposed. Quartz overgrowths were rare.

The sandy shale contains abundant carbonate clasts and is commonly chertified. Two chert-bearing samples (LMSHRl12, from the Adolphus quadrangle, and LMSHK222 from the Dunnville quadrangle) were determined to be limestones and were excluded from the the analysis of shale geochemistry. The dolomite occurs as secondary rhombs.

Samples of Upper Mississippian shale commonly consisted of interlaminated clay, siltstone and sandstone. The silt and sand layers averaged about a millimeter in thickness. Sand layers were commonly well-sorted and relatively coarse (0.1-0.2 $\mathrm{mm}$ grain diameter). Some samples exhibited abundant floating sand grains. The sand grains, in general, were subangular to subrounded and 
consisted of both strained and unstrained quartz, feldspar (including microcline), clay pellets, rock fragments (including some metamorphic-metavolcanic ones), and minor muscovite, chlorite, altered biotite, glauconite, amphibole, opaque and non-opaque heavy minerals (including pyrite), chert, and fine-grained ribbons of organic (?) material. Rarely, a detrital grain of "tan" chert similar to that seen in the Upper Mississipian limestones (Connor, 1981) was present, and samples UMSHJ121 and UMSHJ122 (from the Millerstown quadrangle) contained chert cement. In eastern Kentucky, these rocks were more texturally varied than in western Kentucky. Most samples were silty or sandy claystone and some so poorly sorted as to be graywackes. In many samples, the detritus was mainly poorly sorted ranging in size from fine silt to very coarse sand (over half millimeter in diameter). The larger grains tended to be very well-rounded.

Many samples of Upper Mississippian shale were visibly weathered, and five were judged to be true soils and, hence, deleted from the geochemical analysis. These samples were UMSHQ122, from the Honey Grove quadrangle; UMSHR 212 , Rockfield quadrangle; UMSHJ212, Cub Run quadrangle; UMSHL2ll, Bighill quadrangle; UMSHE2ll, Wrigley quadrangle. Such samples exhibited broken or chaotic textures. Some contained abundant unsorted, largely angular quartz grains. Bedding in some was absent and in others it was present but in seemingly randomly oriented fragments with abundant authigenic clay "skins" on cross-cutting joints. Iron-oxide staining was common. Two additional samples, collected from bedded sequences, also exhibited some of these features suggesting that they may be paleosols. Sample UNSHTll2 (Barthell quadrangle) consisted of heavily iron-stained, plastic clay similar in color and texture to a B-horizon soil. Sample UMSHE122 (Portsmouth quadrangle) was brecciated and iron-stained.

Shale of Pennsylvanian age commonly consisted of interlaminated claystone and poorly sorted, argillaceous siltstone. Many contained floating, sand-sized quartz. Sample PSH-Hl2l (Salem quadrangle) was a fine-grained sandstone and PSH-H222 (Shetlerville quadrangle) was a texturally mature, silicified siltstone. They were excluded from the geochemical evaluation.

Framework grains included abundant monocrystalline quartz and, in some samples, clay pellets or pelitic rock fragments. Less common were plagioclase, rnicrocline, schist, and stretched metaquartzite. Authigenic quartz was locally present. Muscovite was the common mica but much bleached biotite was locally present. Chlorite was rare, as were glauconite, hornblende, chert, and zircon. Opaque minerals include much fine-grained, disseminated hematite, perhaps largely from weathering. Leucoxene and pyrite were rare.

Samples PSH-L21l and PSH-L222 (Parrot quadrangle) were so weathered as to constitute C-zone soils, and PSH-F2ll and PSH-F2l2 were, like UMSHTll2 And UMSHE 122 from the Upper llississippian, fragmented and iron-stained and might be paleosols. These last four samples were excluded from the geochemical evaluation.

\section{GEOCHEMICAL VARIABILITY}

The analysis of geochemical shale unit are given in tables variance and geochemical summaries for each $5 A-5 D$ and $6 A-6 D$, respectively. In the 
organic-rich shale (Chattanooga and lithic equivalents), a variety of elements exhibit statistically significant regional (between-areas) variance. Samples from westernmost Kentucky (the Briensburg and Eddyville quadrangles) are distinctly high in $\mathrm{B}$ and distinctly low in organic $\mathrm{C}$ and $\mathrm{Cu}$. $\mathrm{Pb}$ appears to be distinctly low in samples from northeasternmost Kentucky (the Burtonville and Manchester Islands quadrangles). P205, Ni, Mn, Co, and organic C tend to be high over the Cincinnati arch, whereas $B$ is relatively low over the arch. Organic $C$ constitutes about 5-13 percent by weight of the rock (table 6A, Part II). Conant and Swanson (1961, p. 13) found that the organic content ranges from 5-25 percent over the entire basin of deposition. Leventhal (1978) reported that the organic matter was derived from both marine algae and lignin-type terrestrial materials (vitrinite).

The analysis of variance of the shale and siltstone in the Lower Mississippian is given in table 5B. Eight of 23 constituents exhibit regionally significant components of variation. This variation is summarized in table $6 \mathrm{~B}$, Part II. If calcareous rocks from the Amandaville-Wolf Creek Dam area are excluded from the analysis, the regional differences in $\mathrm{CaO}, \mathrm{MgO}$ and $\mathrm{Sr}$ are greatly subdued; SiO2 and $\mathrm{Zr}$ are high and $\mathrm{K} 20, \mathrm{~B}$, and Co are low in samples from the northeast (head of Grassy, Wesleyville and Brushart quadrangles), reflecting the more continental aspect of these rocks.

Three samples from the Holland quadrangle in south-central Kentucky (table 6, Part I, footnote 5) are unusually high in $\mathrm{Ni}$ compared to an expected value of $49 \mathrm{ppm}$. Samples of highly chertified Lower Mississippian limestone from the same general area were also elevated in $\mathrm{Ni}$ (Connor, 1981, p. 9). Further, $\mathrm{Ni}$ in the underlying organic-rich shale also appears to elevated in this general part of the State (table 6A, Part II); that is, over the Cincinnati arch. The common sedimentary ore elements, $\mathrm{Cu}$ and $\mathrm{Pb}$, are not unusually high here and the origin of the high $\mathrm{Ni}$ rocks remains obscure.

The analysis of variance of shale of Upper Mississippian age is shown in table 5C. Five constituents exhibit statistically significant regional variation -- $\mathrm{FeO}, \mathrm{Na} 20, \mathrm{P} 205, \mathrm{Ba}$ and $\mathrm{Cr}$. The high concentration of $\mathrm{FeO}$ in the Cub Run-Millerstown and Barthel1-Sawyer areas (table 6C, Part II) may reflect a greater abundance of iron-bearing clay (chlorite?) in rocks from those quadrangles. Variation in $\mathrm{Na} 20$ probably reflects a greater content of plagioclase in samples from the Barthell quadrangle. P205 is also distinctly high in these samples and likely represents an increase in apatite. Ba increases systematically from southwest to northeast and conceivably reflects an increase in potassium-feldspar towards source.

The analysis of variance of Pennsylvanian shale is given in table 5D. Five constituents exhibit statistically significant regional variation -- $\mathrm{CaO}, \mathrm{Na} 20$, $\mathrm{B}$, Co and $\mathrm{Pb}$. $\mathrm{Ni}$ is significant at the $90 \%$ confidence level. Variation by area (table 6D, Part II) indicates that much of the significance in $\mathrm{CaO}$ and Na20 reflects the relative high concentrations in the slaughters quadrangle. The failure of $\mathrm{A} 1203$ and $\mathrm{K} 20$ to exhibit a similar variation weakly suggests that these samples contain relatively more plagioclase than other Pennsylvanian shales. The samples were taken from the upper part of the Pennsylvanian, near the middle of the Sturgis Formation. 
The geochemical nature of Pennsylvanian shale in Kentucky is demonstrated in figure 6. The frequency distributions in this figure are visual representations of the data in the summary tables. These summaries in turn were organized, in part, according to the statistical tests given in the analysis of variance tables (tables 5A-5D).

The distributions show that, in general, Paleozoic shale in Kentucky is rather geochemically uniform, much more so than the Paleozoic carbonate rocks (Connor, 1981). Whereas the point scatter in the carbonate histograms commonly equalled or exceeded 20-fold, most of the trace elements in the shale exhibit a point scatter at or below 10-fold. Exceptions for shale include such constituents as $\mathrm{MgO}$ and $\mathrm{CaO}$, which reflect a highly erratic distribution of carbonate cement, or constituents such as $\mathrm{Na} 20$ and P205, for which about half the scatter arises from laboratory procedures ( $V(E)$ of equation 2).

Variation not specifically identified in the distributions of figure 6 include variation described by the intermediate-scaled components of equation (2). Such variation commonly accounts for more than half of the total. Differences among samples within localities is uniformly high (V(D)), and differences among localities within quadrangles (V(C)) is high for shale of Upper Mississippian age, but differences among quadrangles within one-degree areas $(V(B))$ are generally low. Presumably, much of the variation at the two lower levels $(V(C)$ and $V(D))$ reflects variation in the clay-quartz ratio among samples and localities. On the whole, the fitted distributions look to be good, except for $\mathrm{CaO}, \mathrm{Na} 2 \mathrm{O}, \mathrm{Hg}$ and $\mathrm{Mn}$, and should prove useful in providing a "first approximation" to the definition of geochemical variability in Paleozoic shale of Kentucky. 


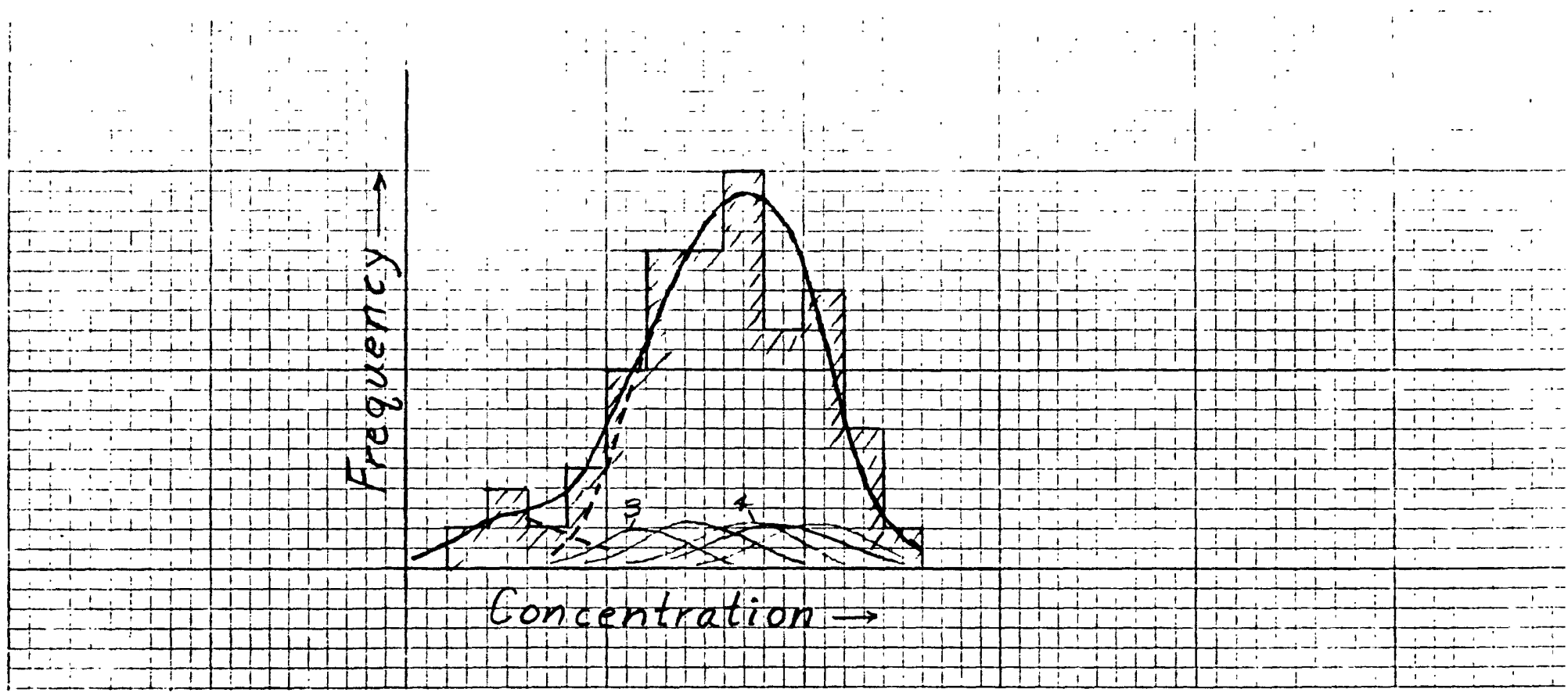

Figure 6.--Explanation of geochemical frequency distributions. The distributions shown on the following pages are meant to be visualizations of the summary data in tables $6 \mathrm{~A}-6 \mathrm{D}$. Histograms for each geochemical variable in each of the four major shale units are outlined by hachures. Each histogram is modeled by a heavy tlack solid line which, in the Lower Mississippian and Pennsylvanian units, is the "sum" of two lithically distinct subpopulations, shown by heavy, dashed, black lines. These subpopulations are listed in Part I of tables 6B (sandy shale--Sdy $\mathrm{Sh}$ in the figure) and 6D (siliceous shale-$\mathrm{Sil} \mathrm{Sh}$ in the figures). Subpopulations shown by the light solid lines are based on the area means of Part II, tables $6 \mathrm{~A}-6 \mathrm{D}$. A number attached to one of these indicates that that line represents more than one such subpopulation. All subpopulations are based on the lognormal model. If a geometric deviation (GD) was not computed for a subpopulation (tables 6A-6D), a GD of 1.5 was assumed. 


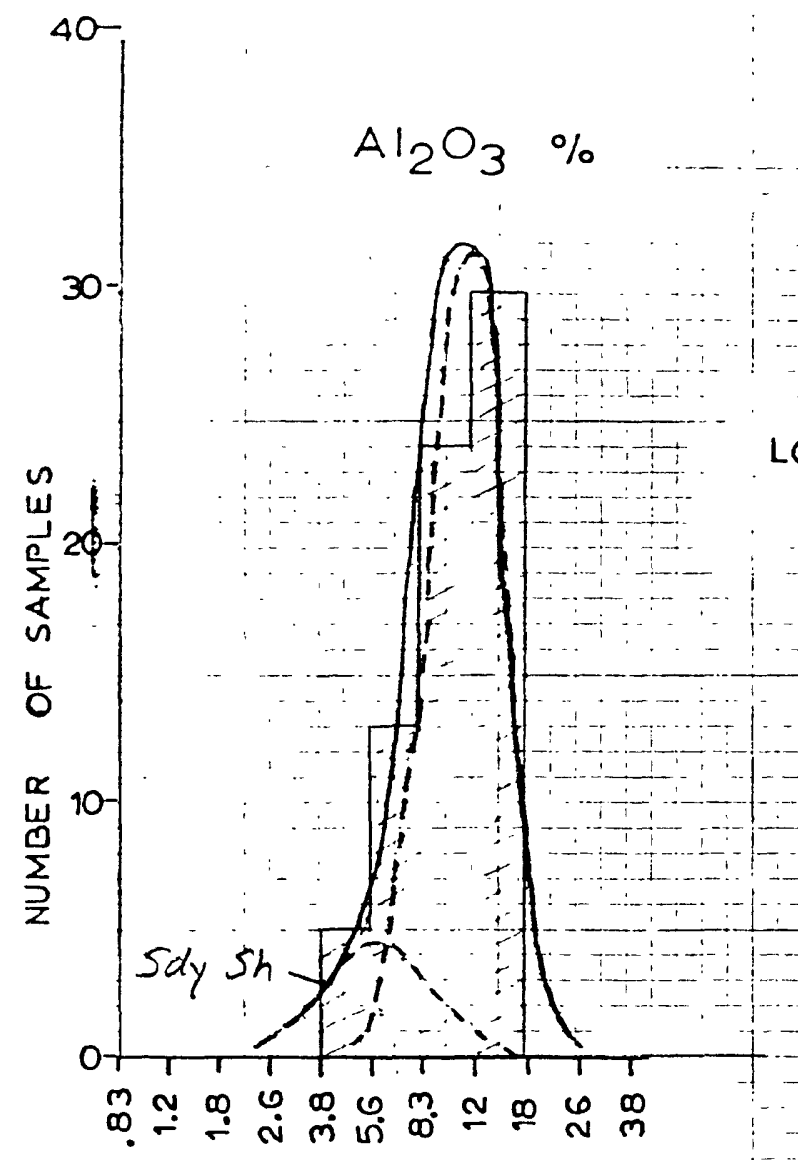

$\mathrm{Fe}_{2} \mathrm{O}_{3} \%$

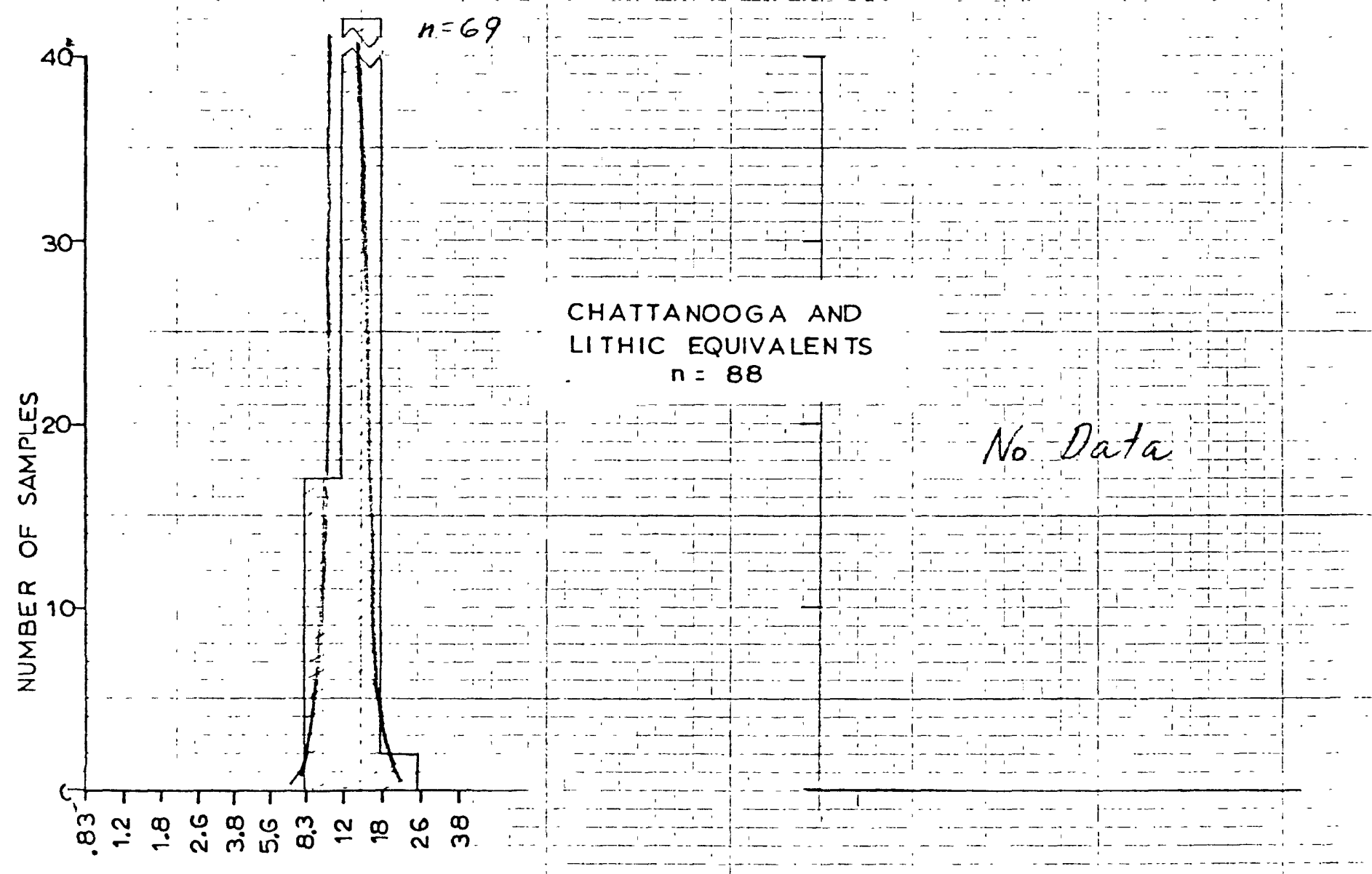



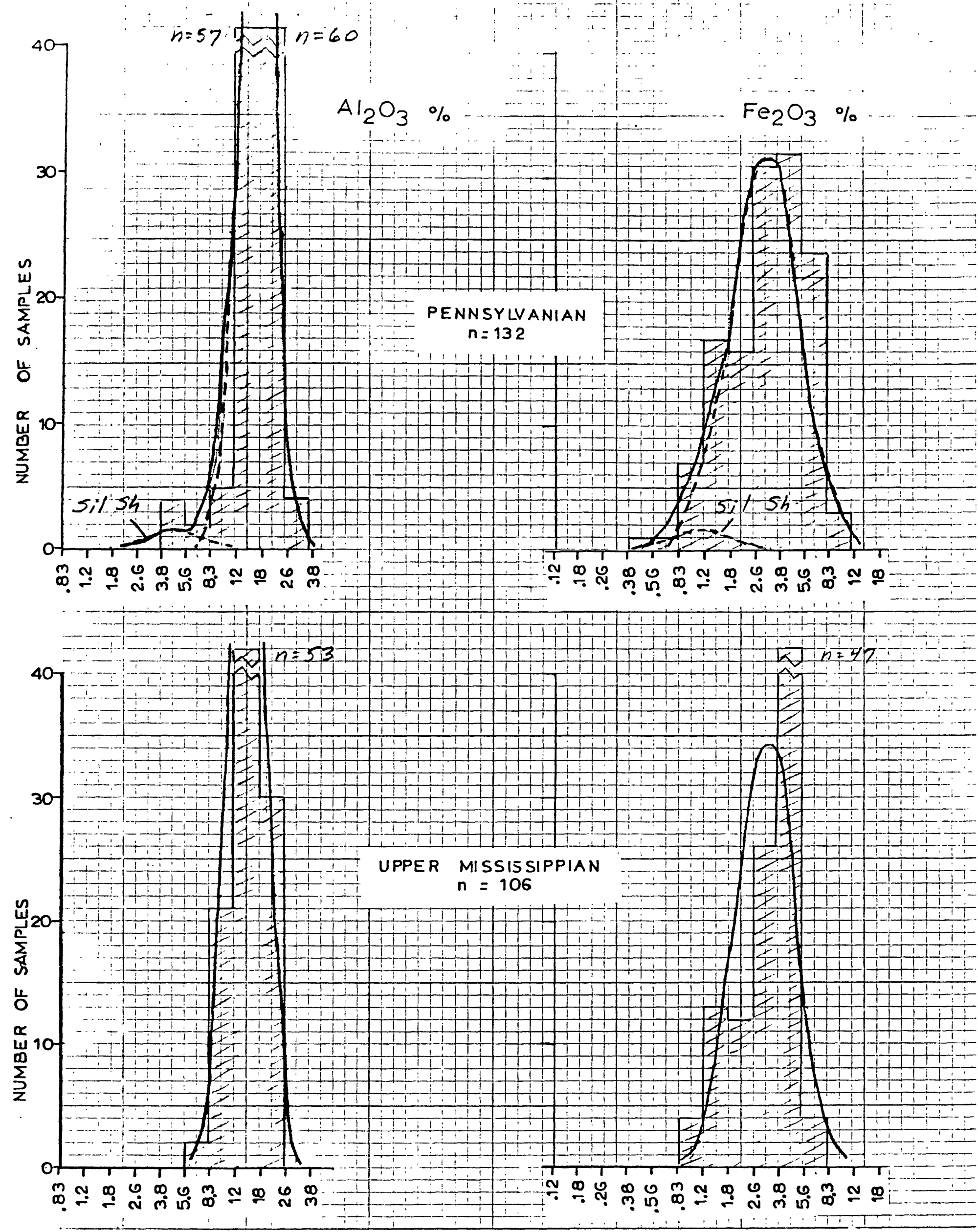

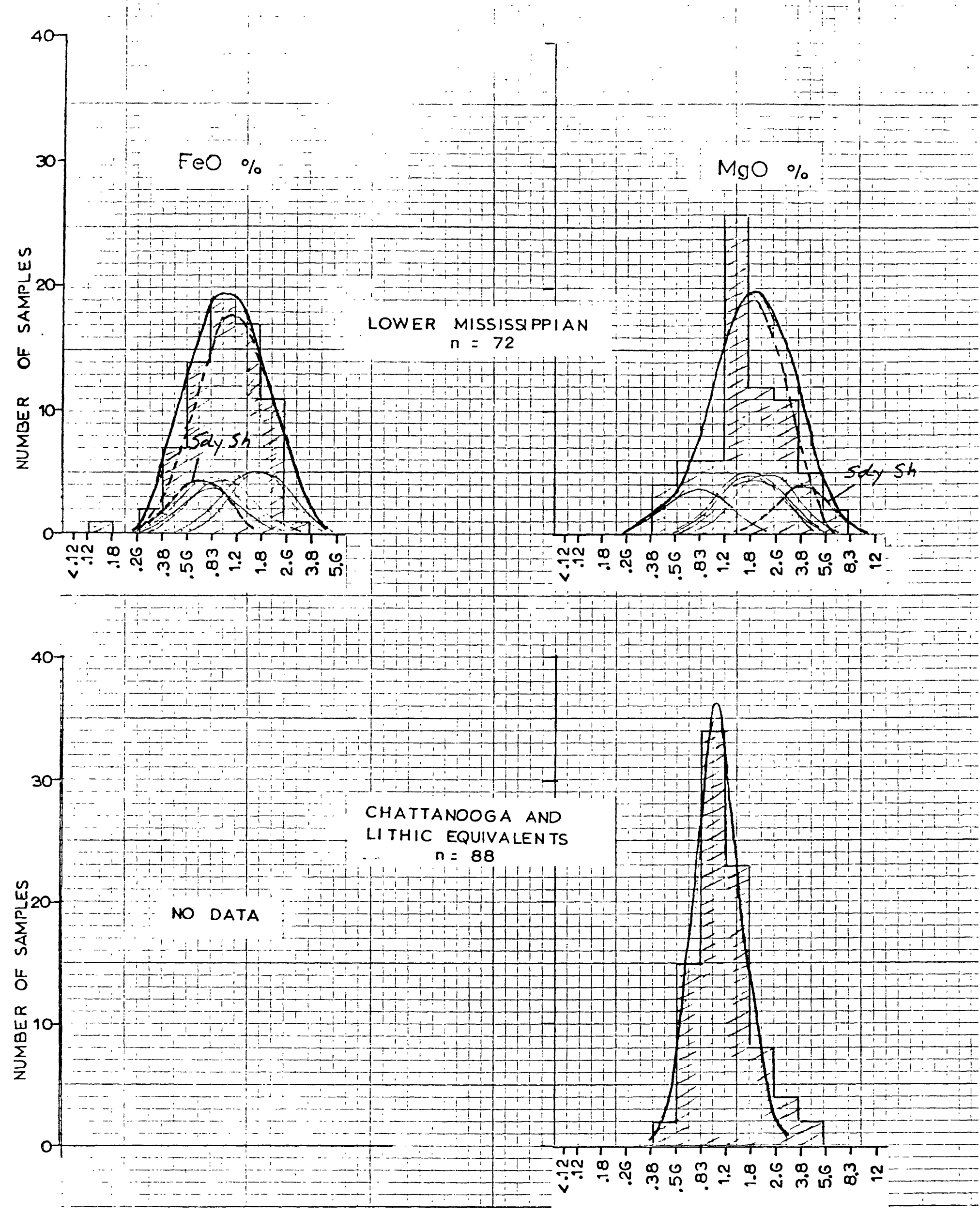

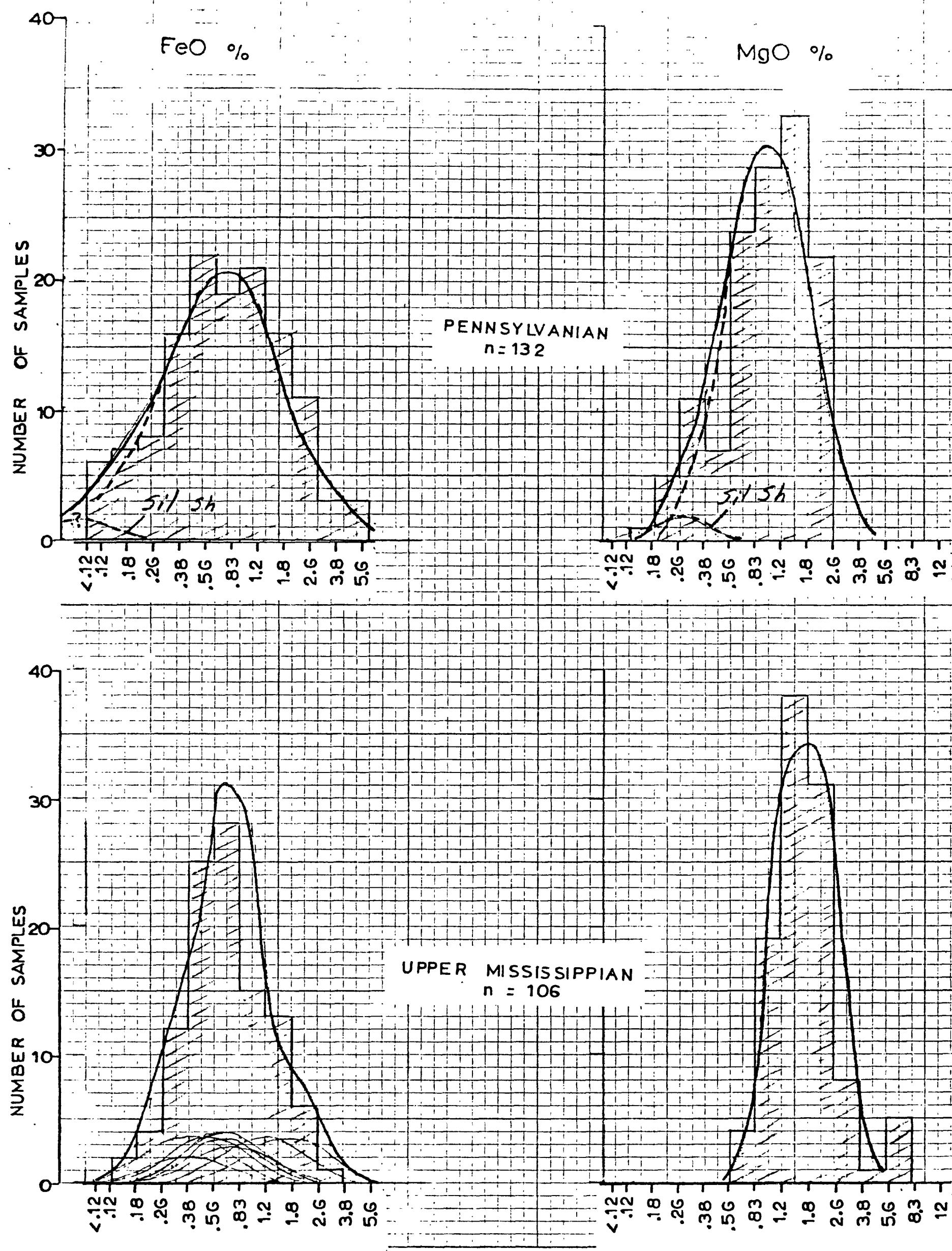






Figure 6.--Continued 

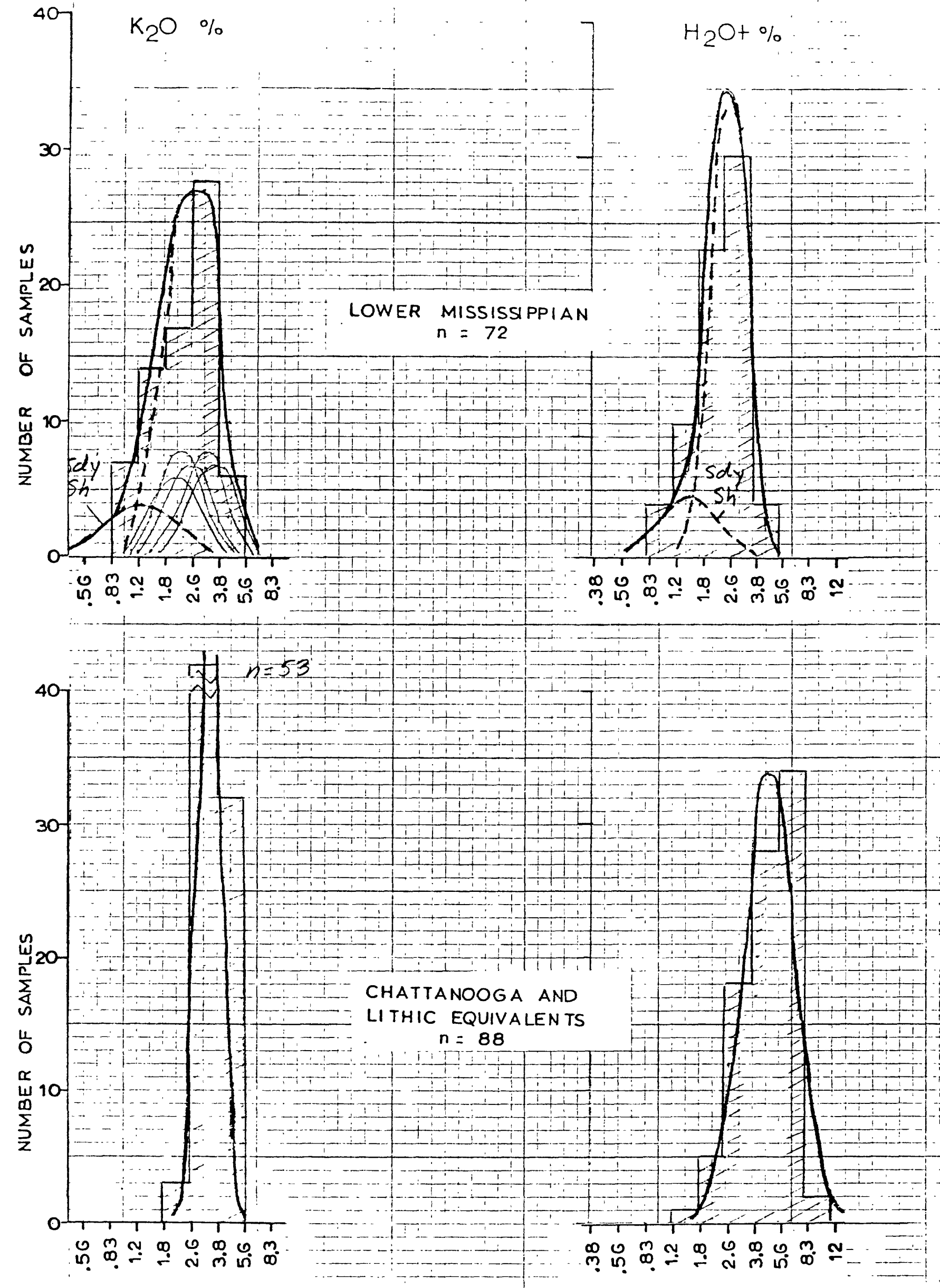

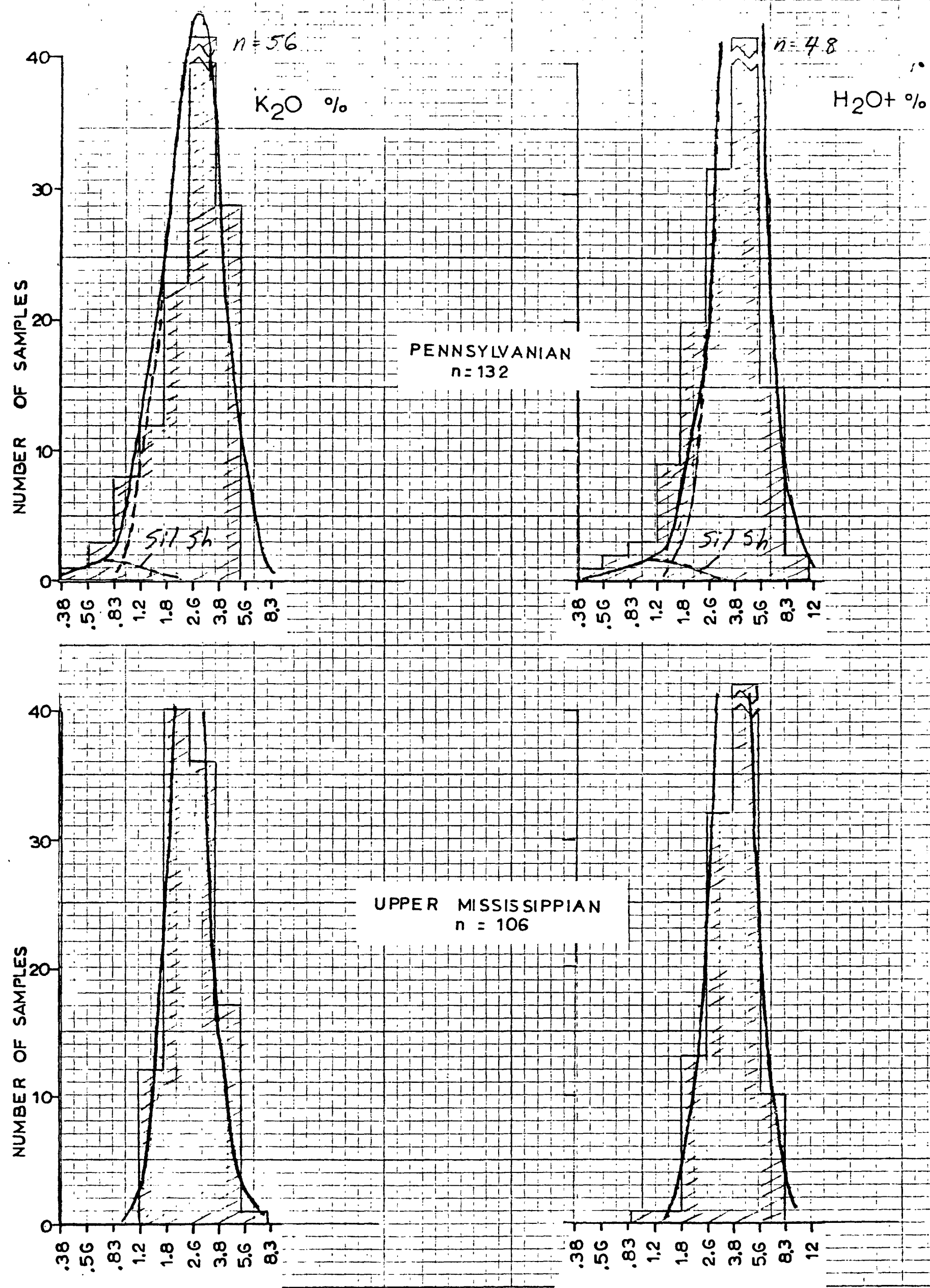



\section{CHATTANOOGA AND LITHIC EQUIVALENTS $n=88$}
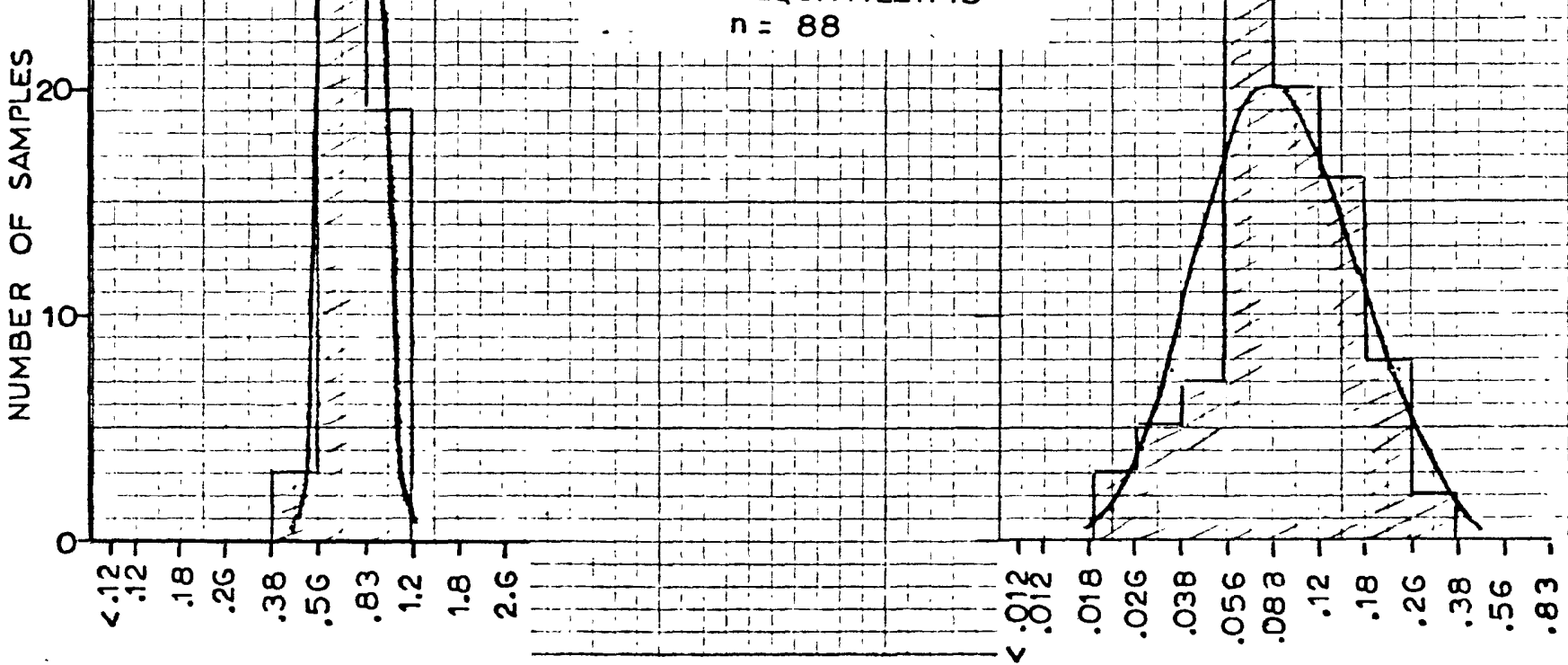


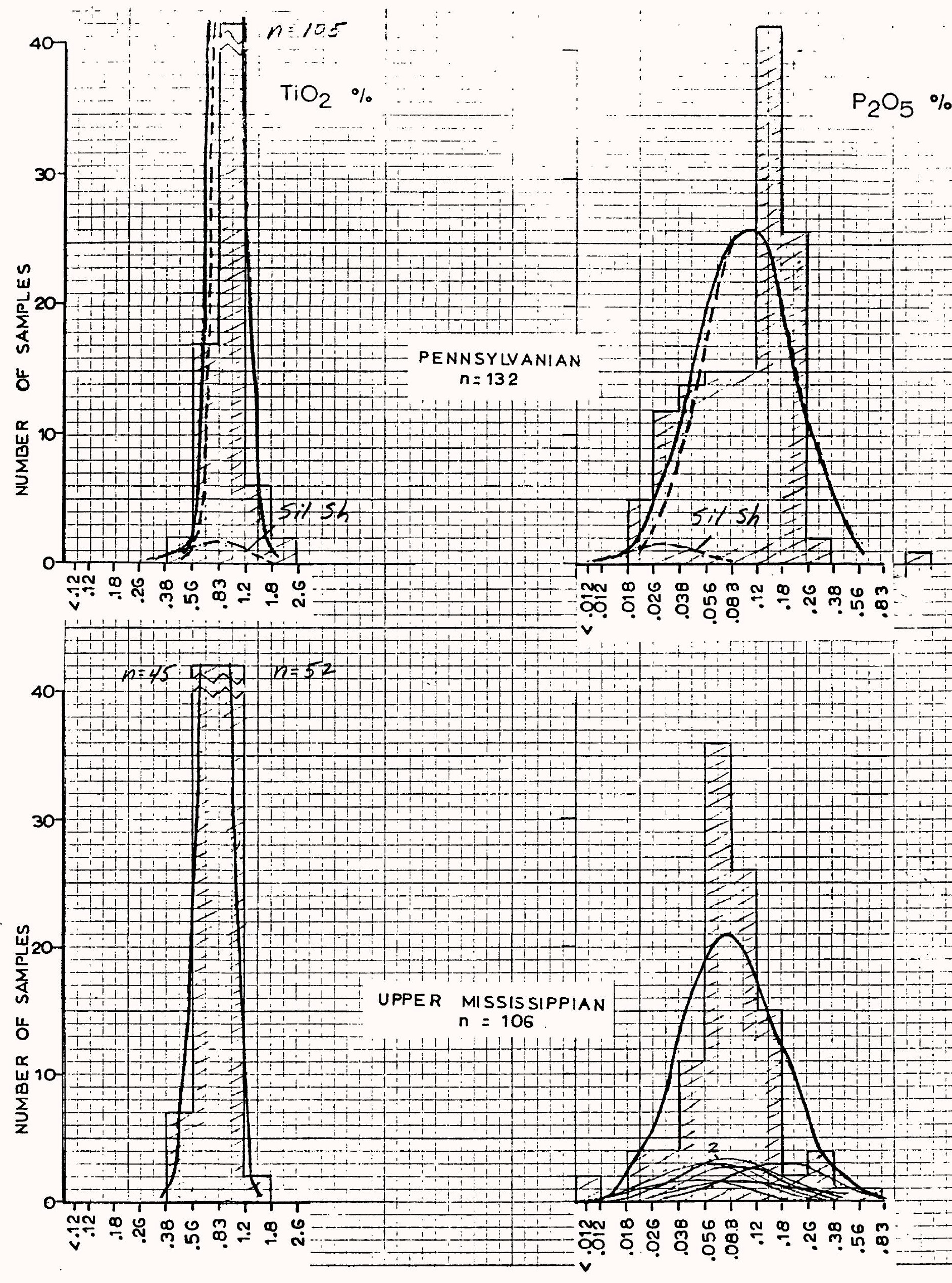




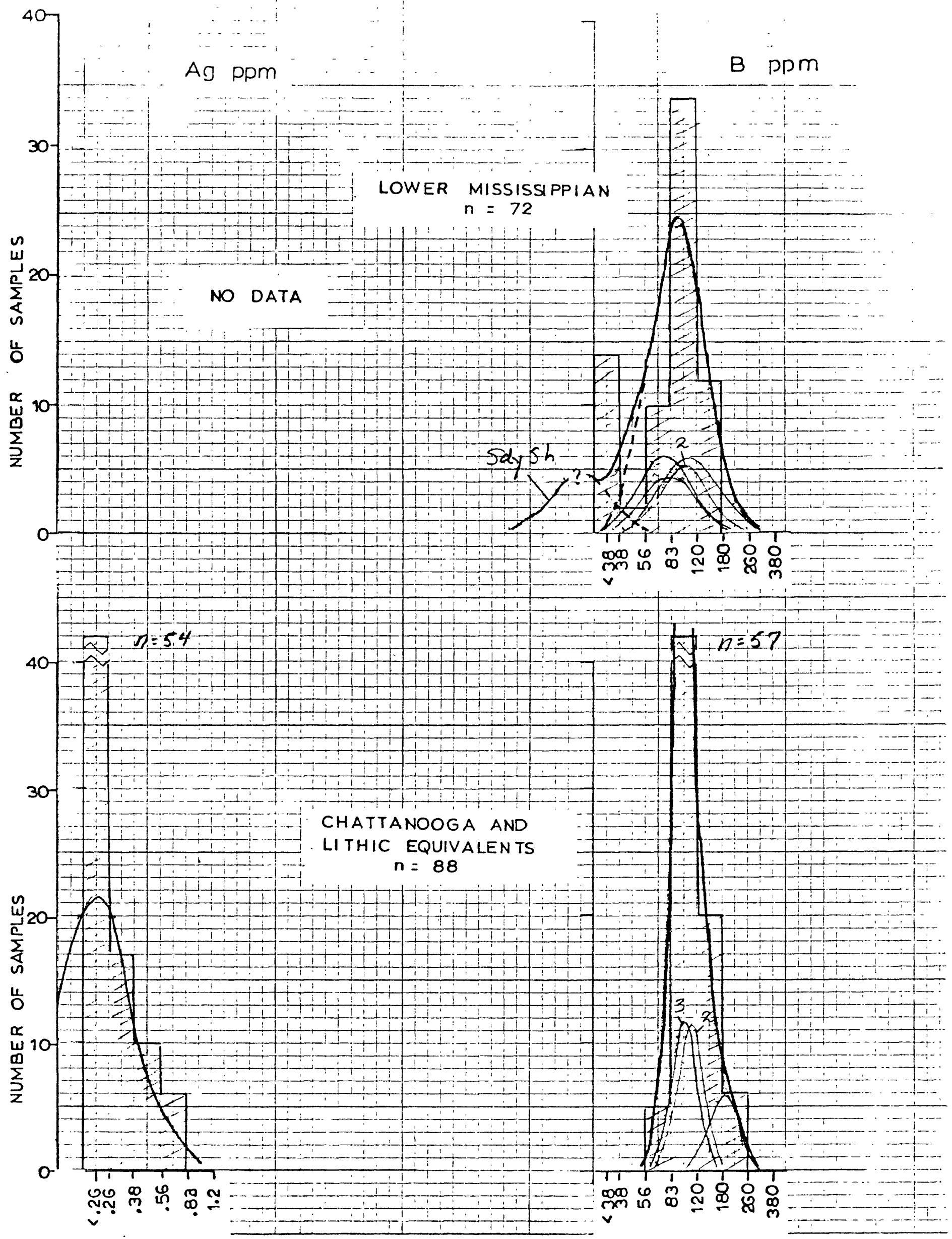

Pigure 6.--Continued 


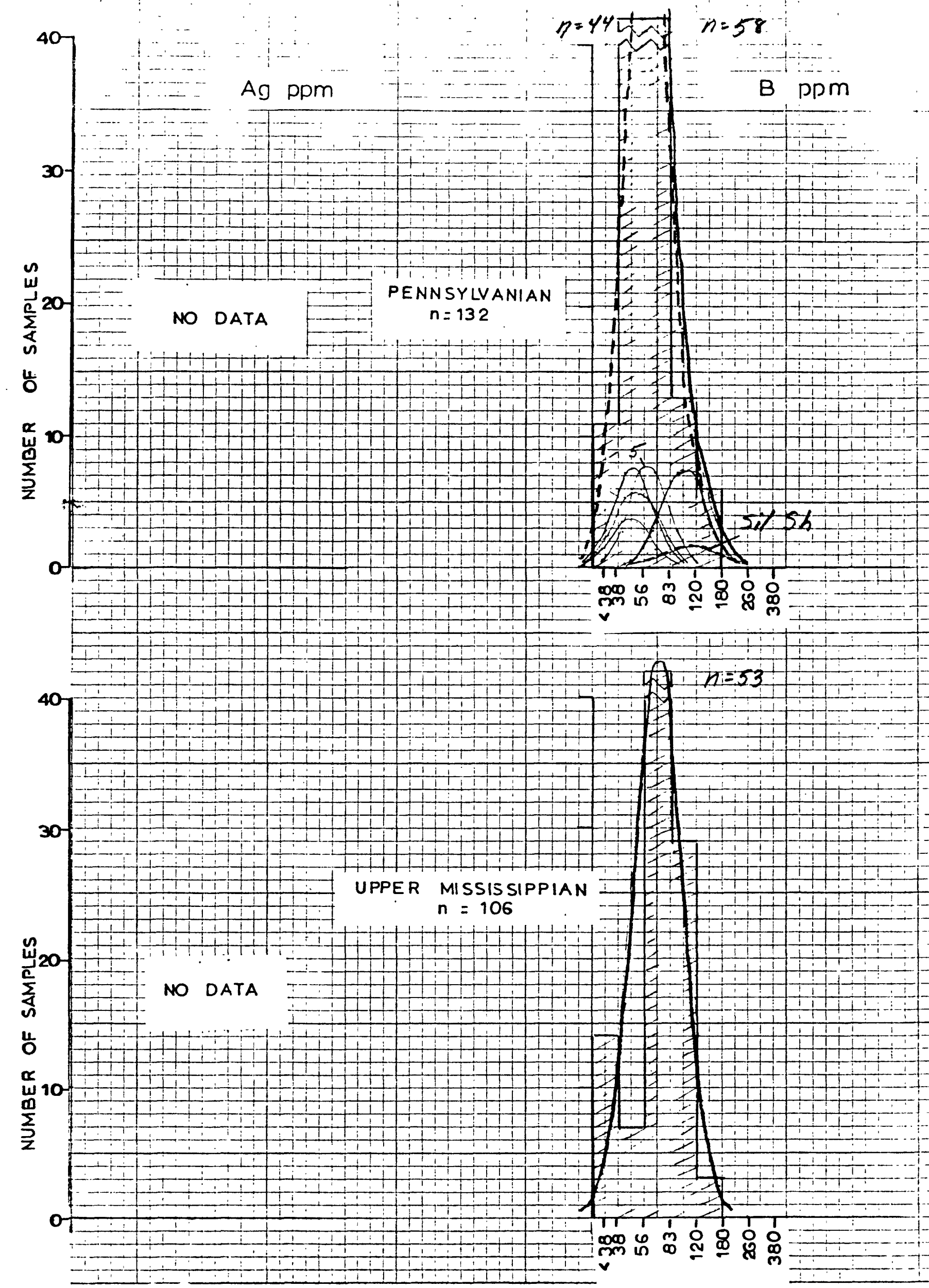




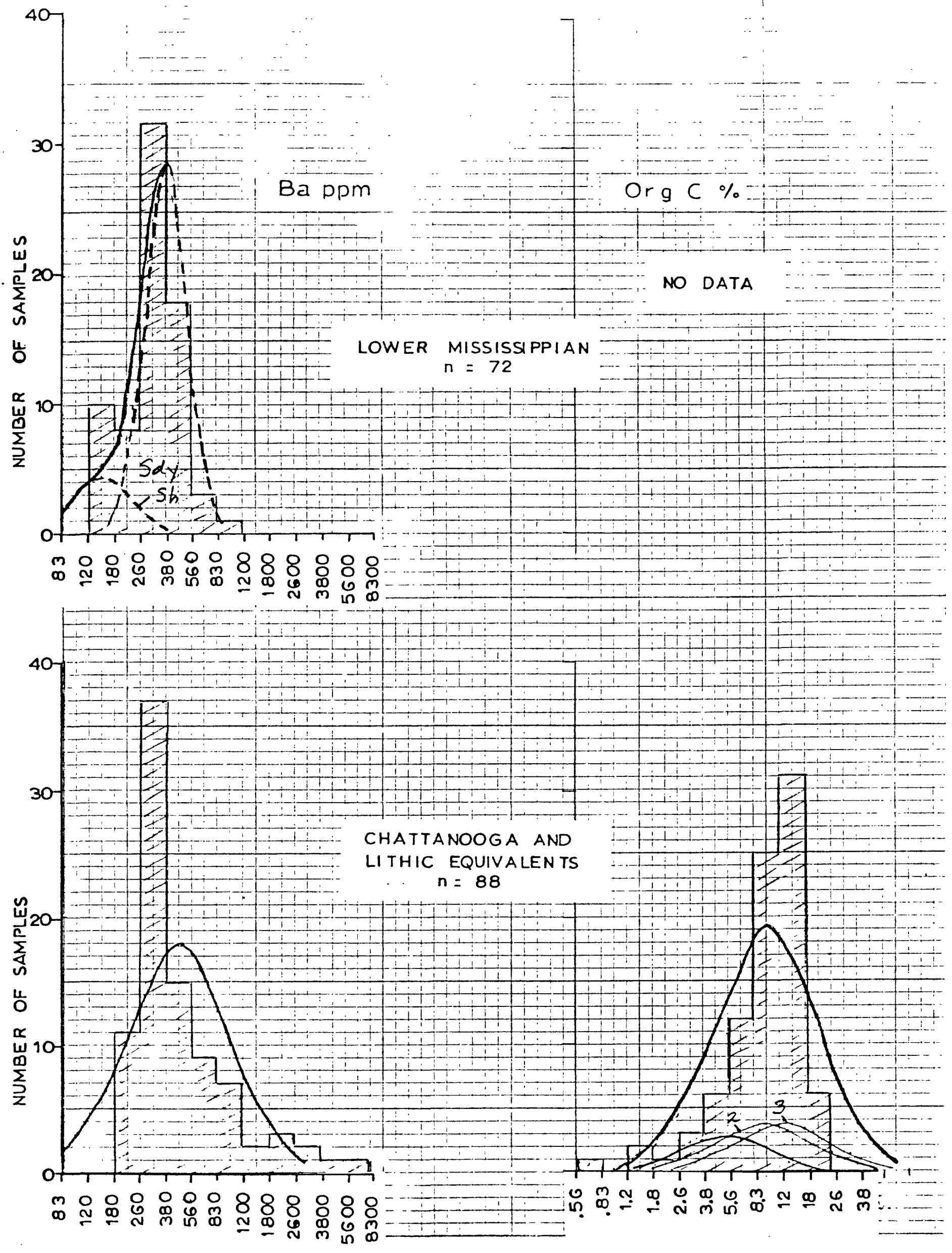

Figur $€$ 6.--Continued 


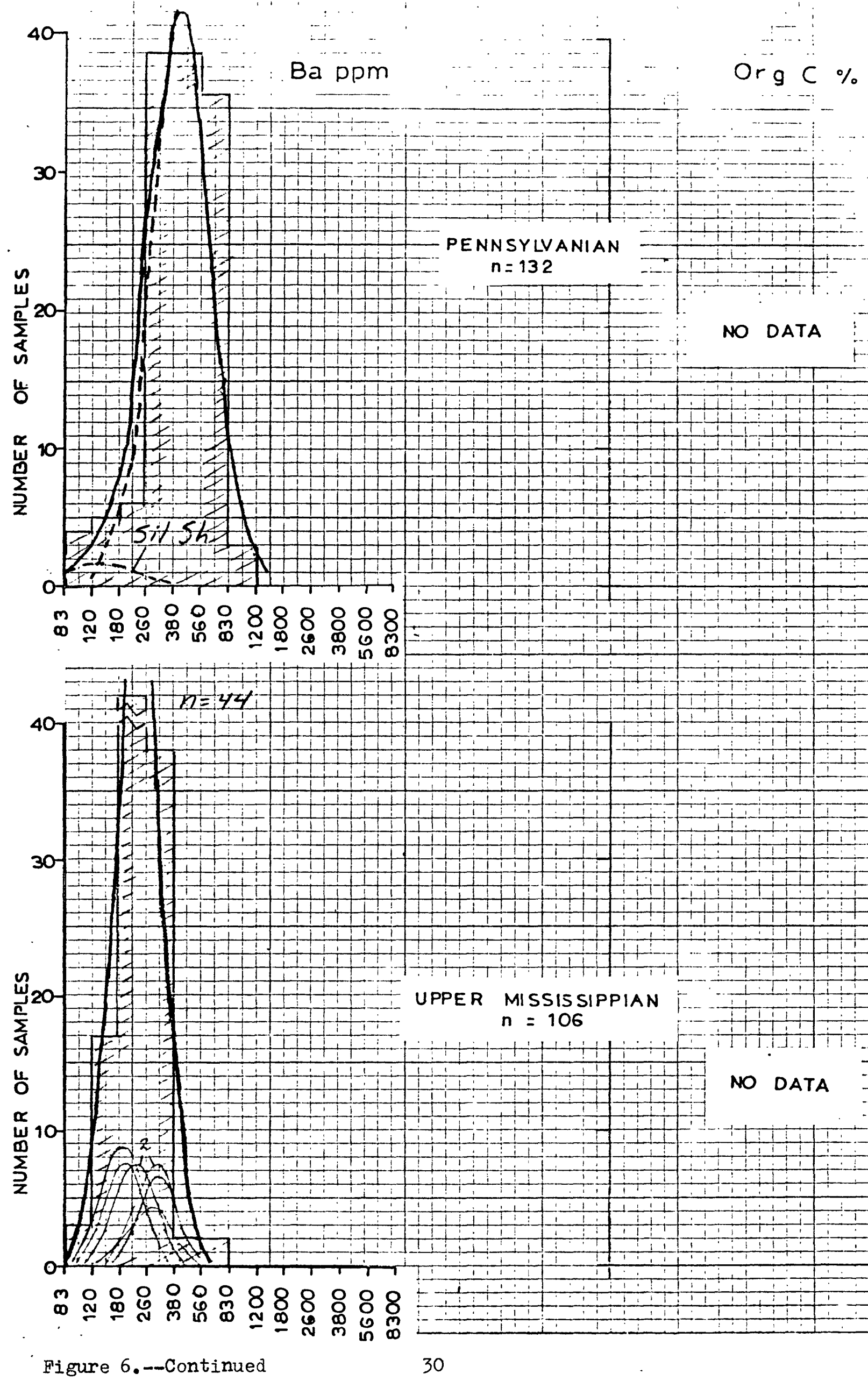



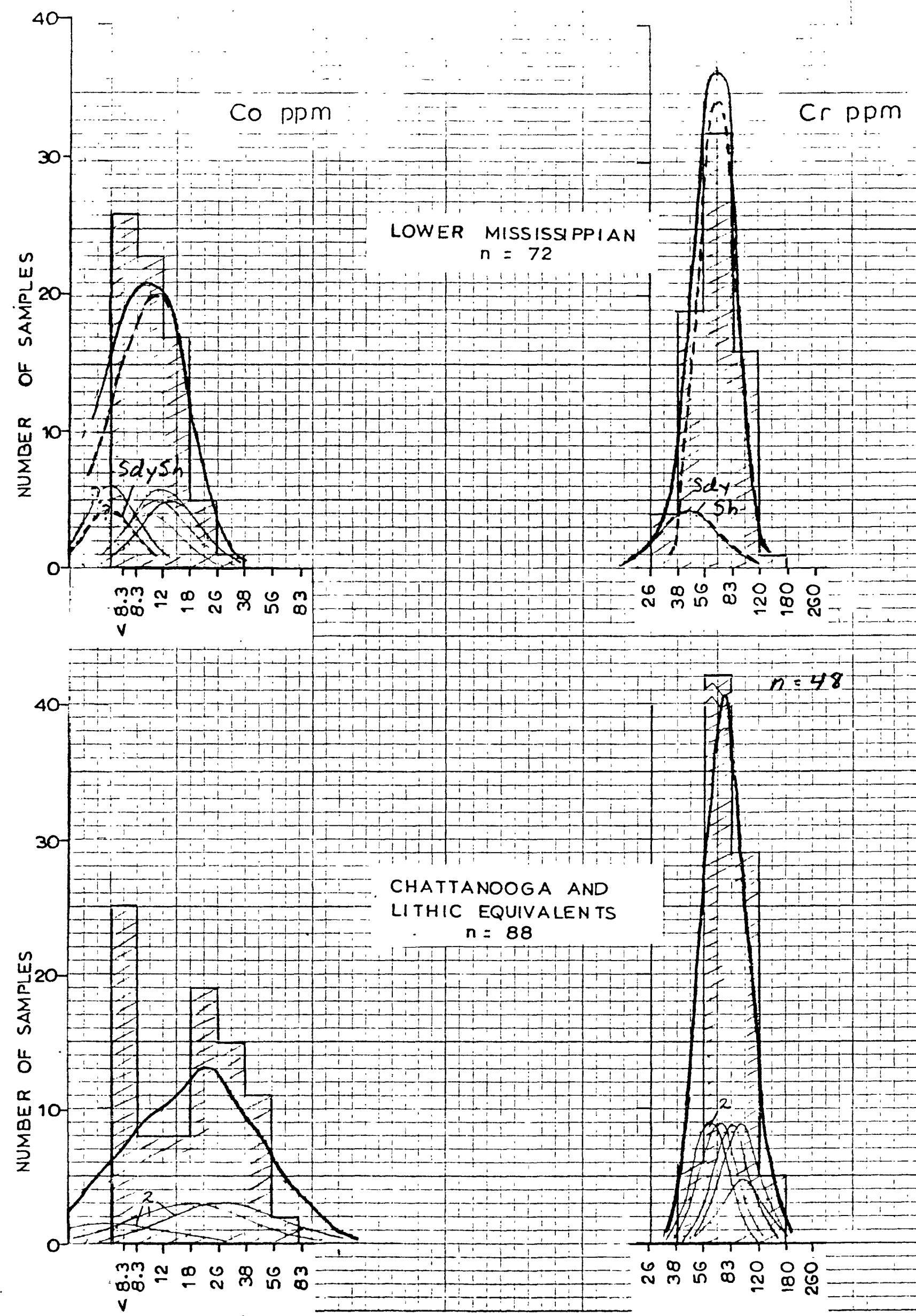

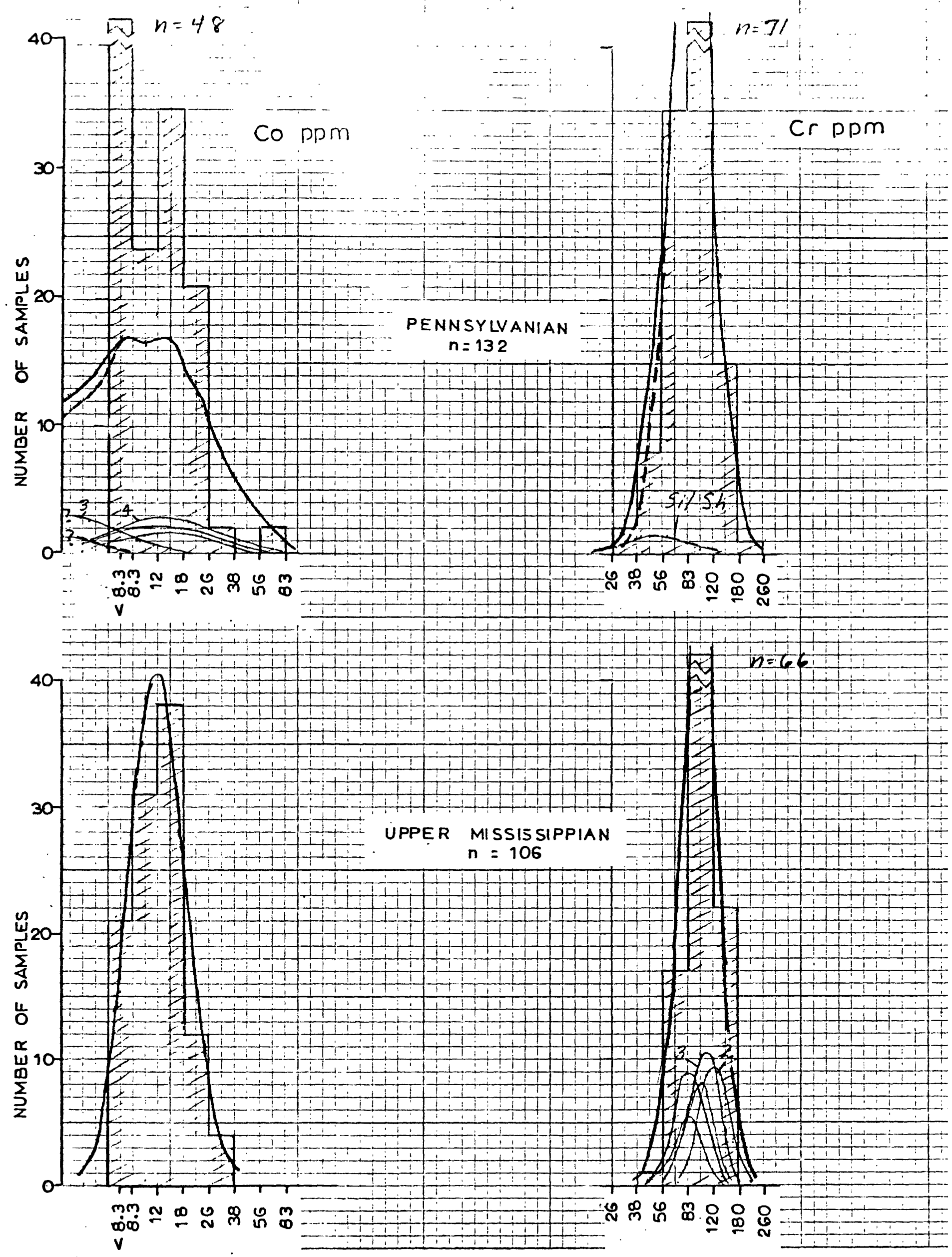

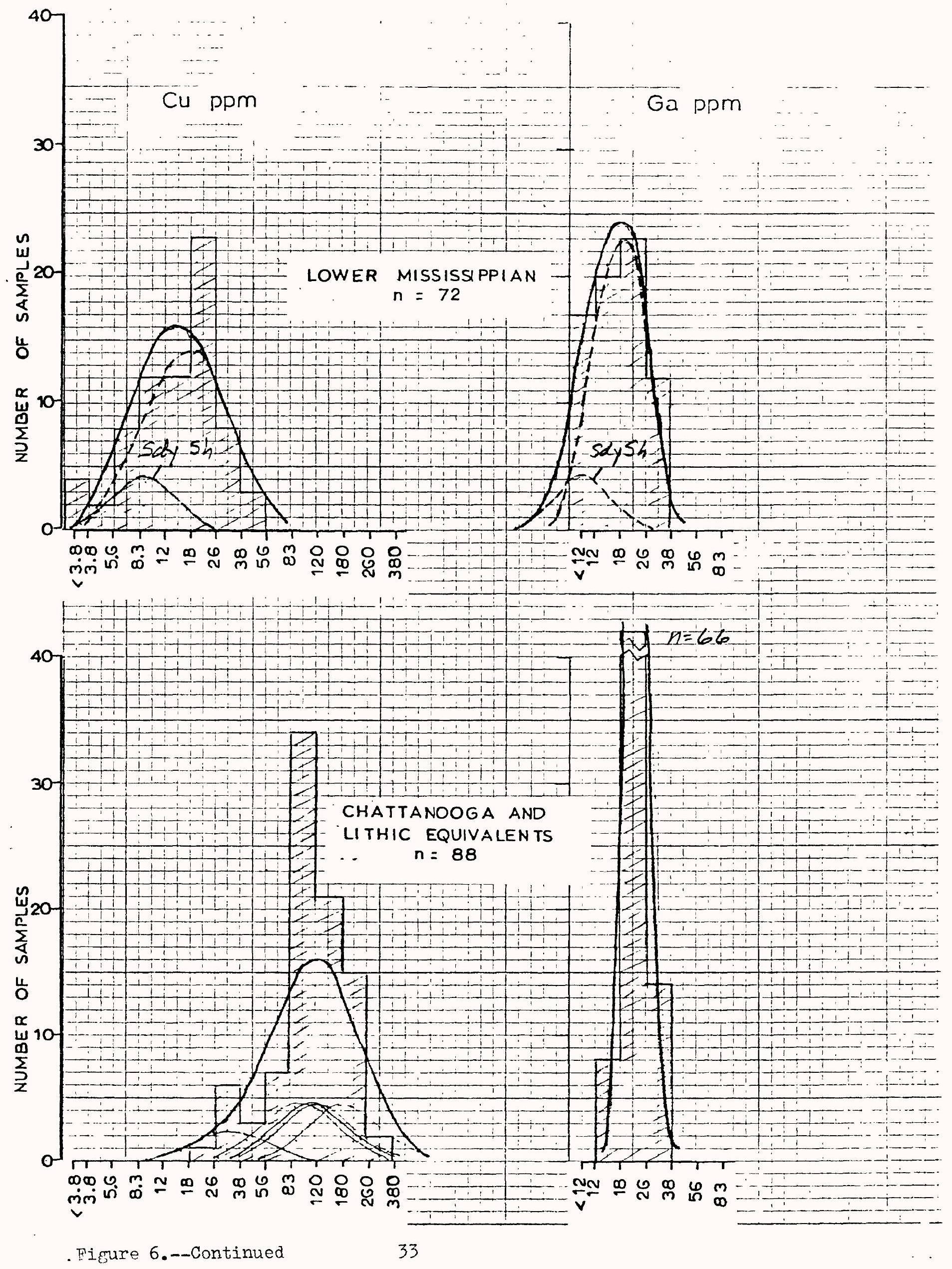

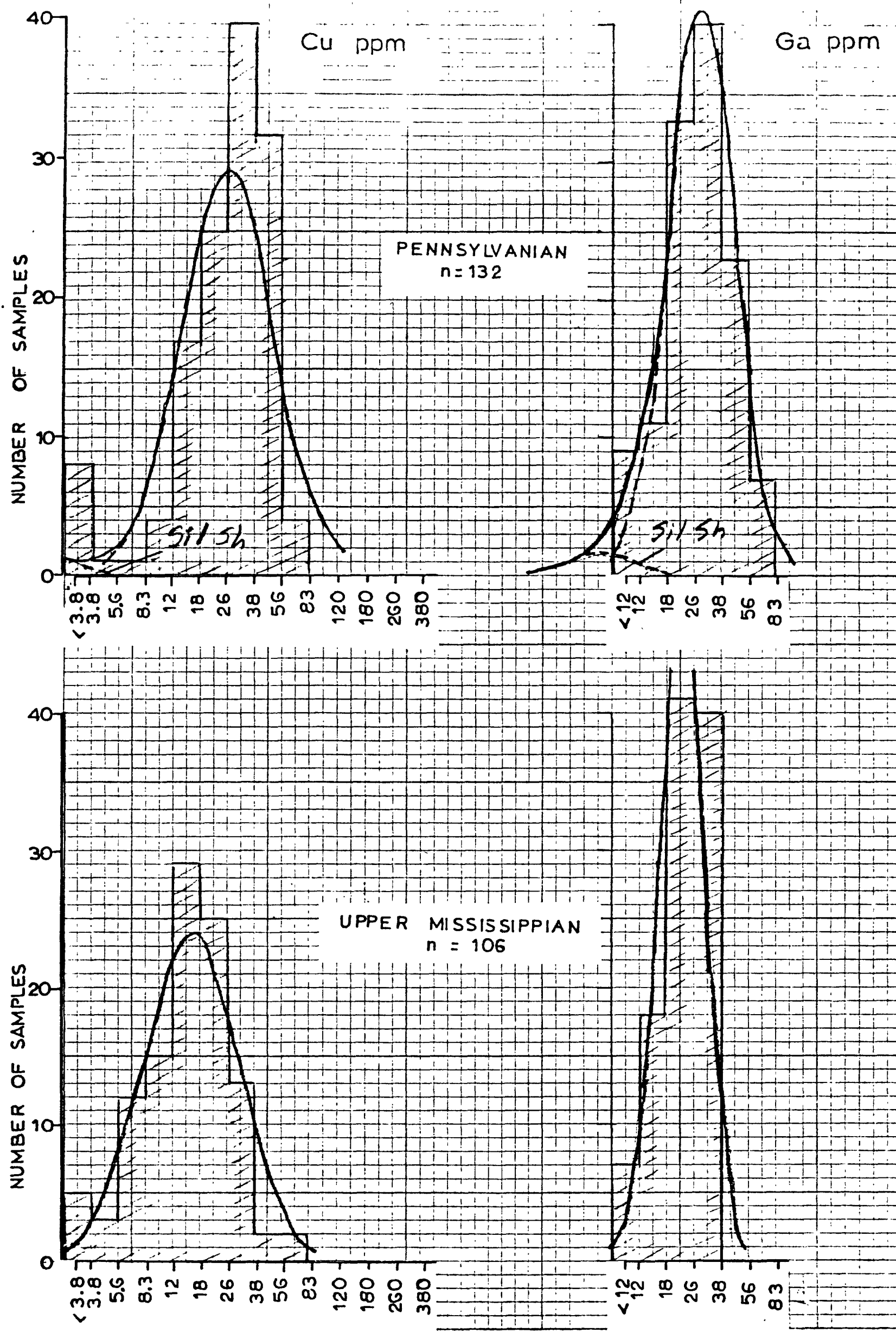

Figure 6.--Continued 


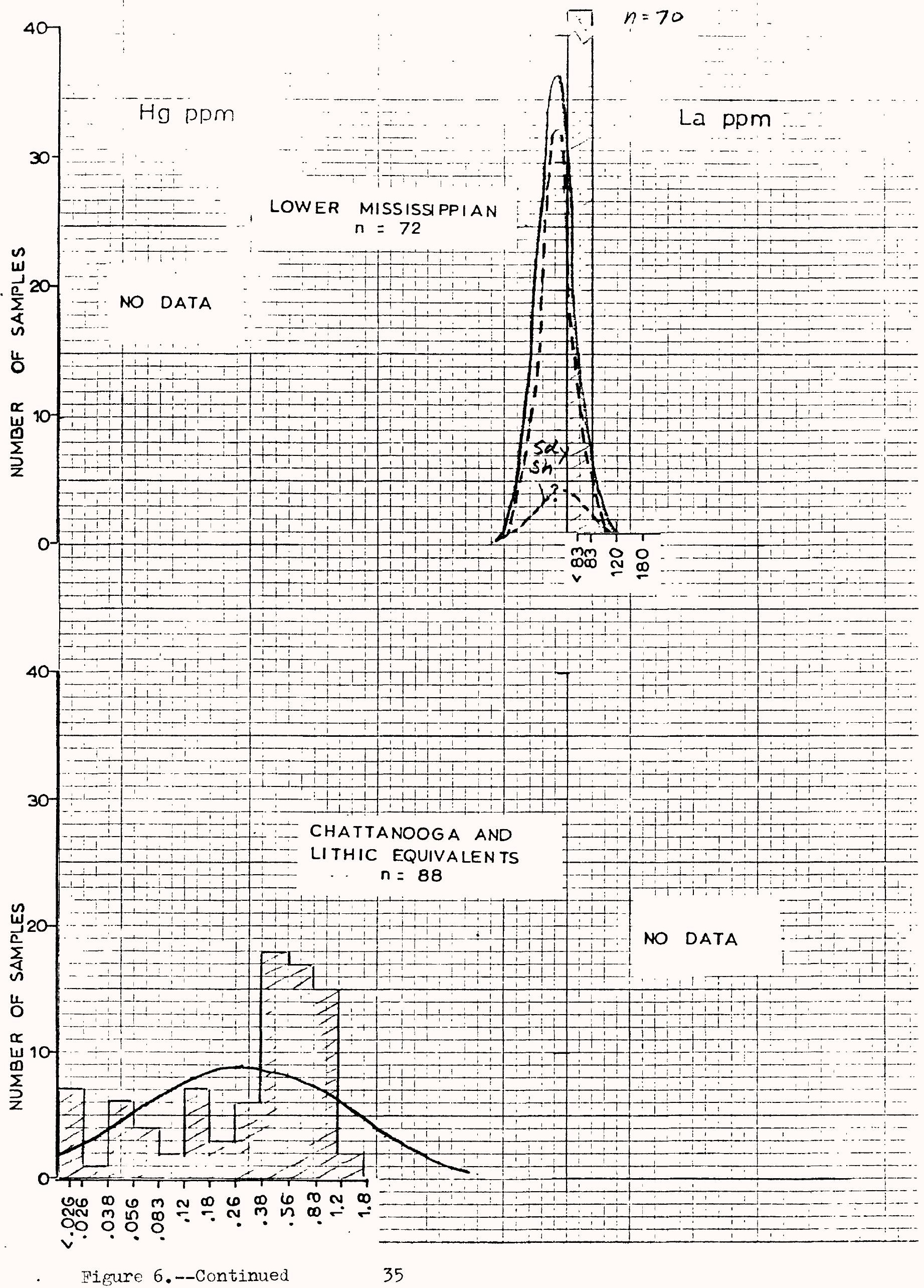




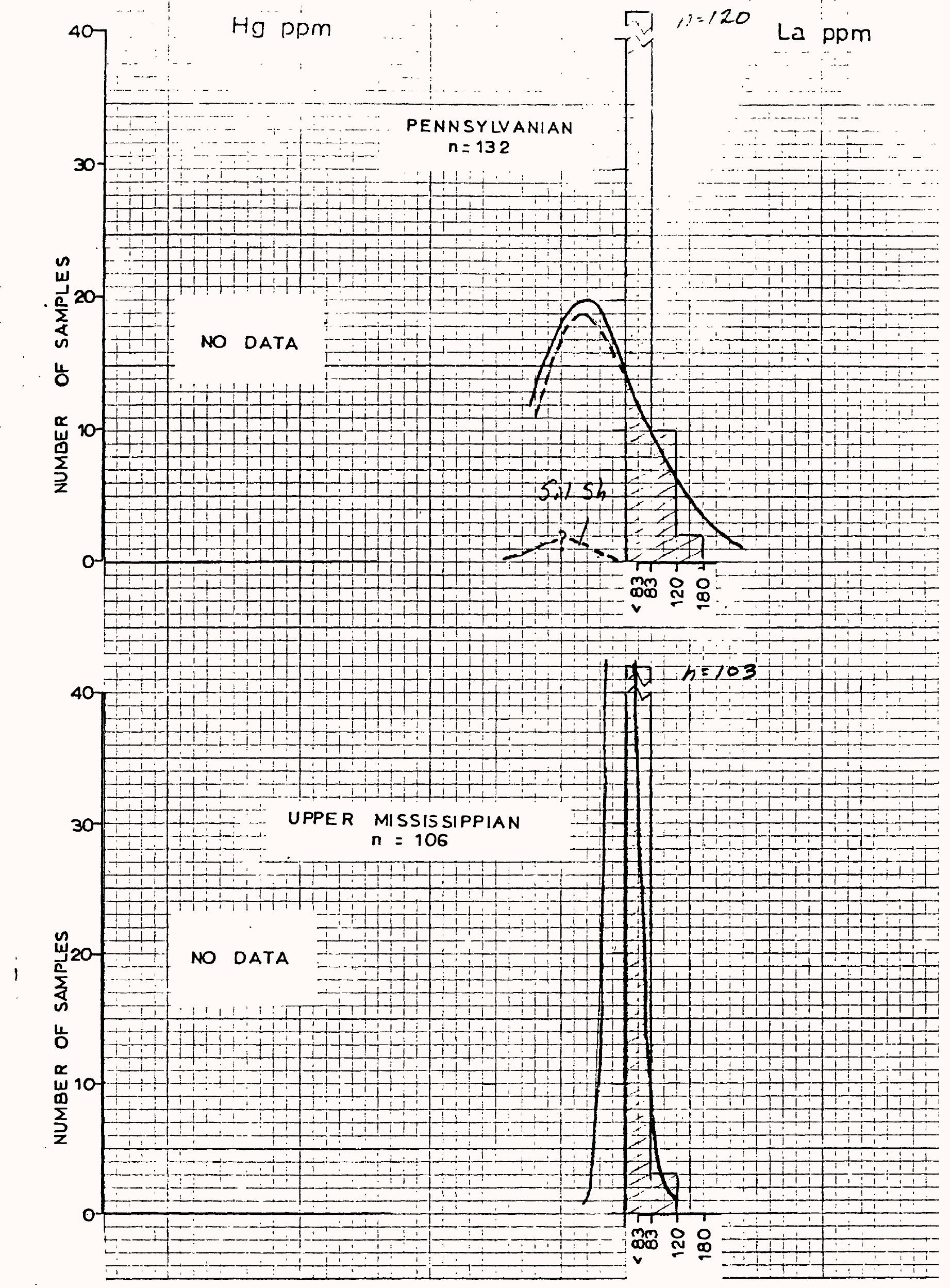



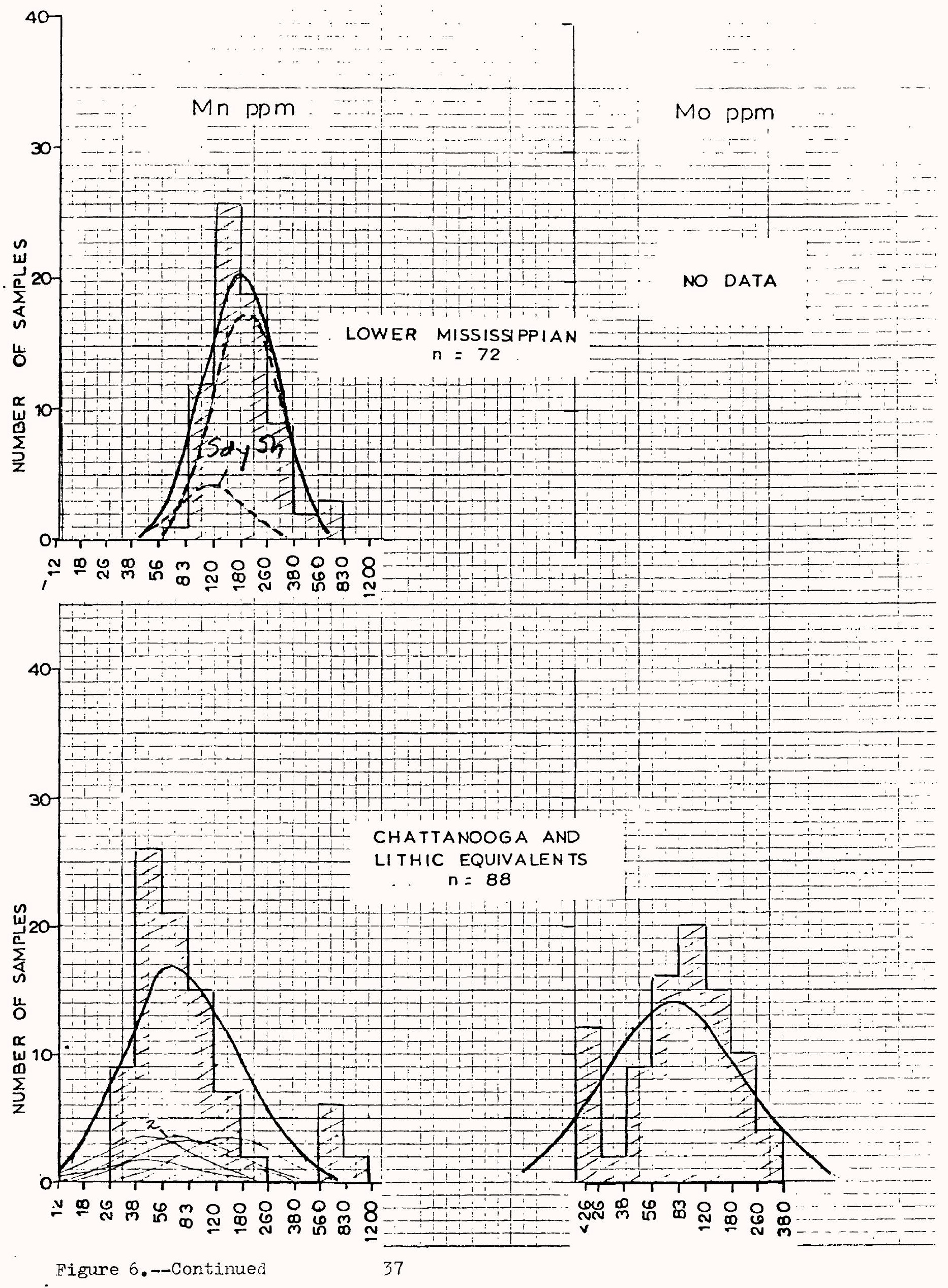

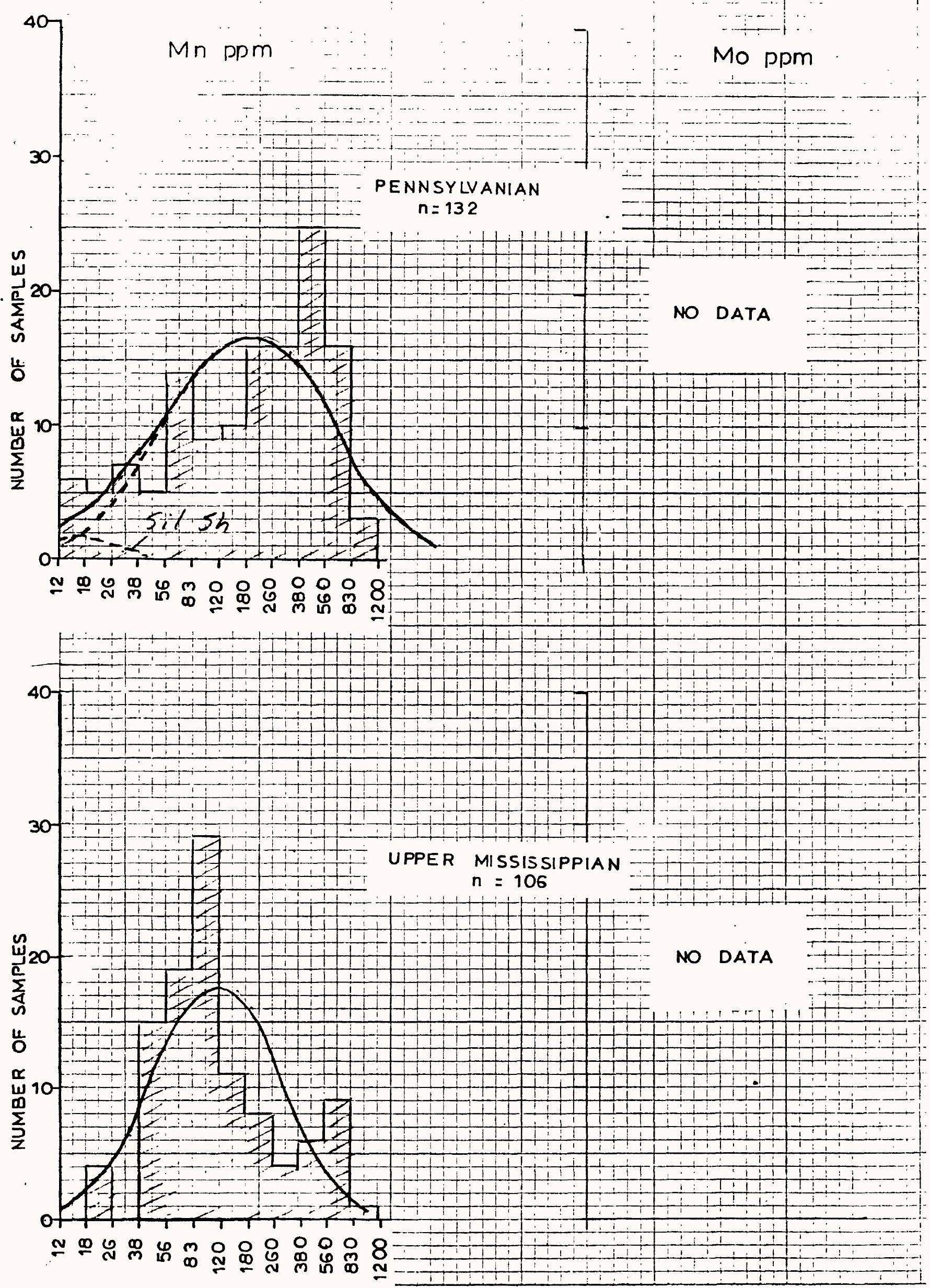

Figure 6.--Continued 


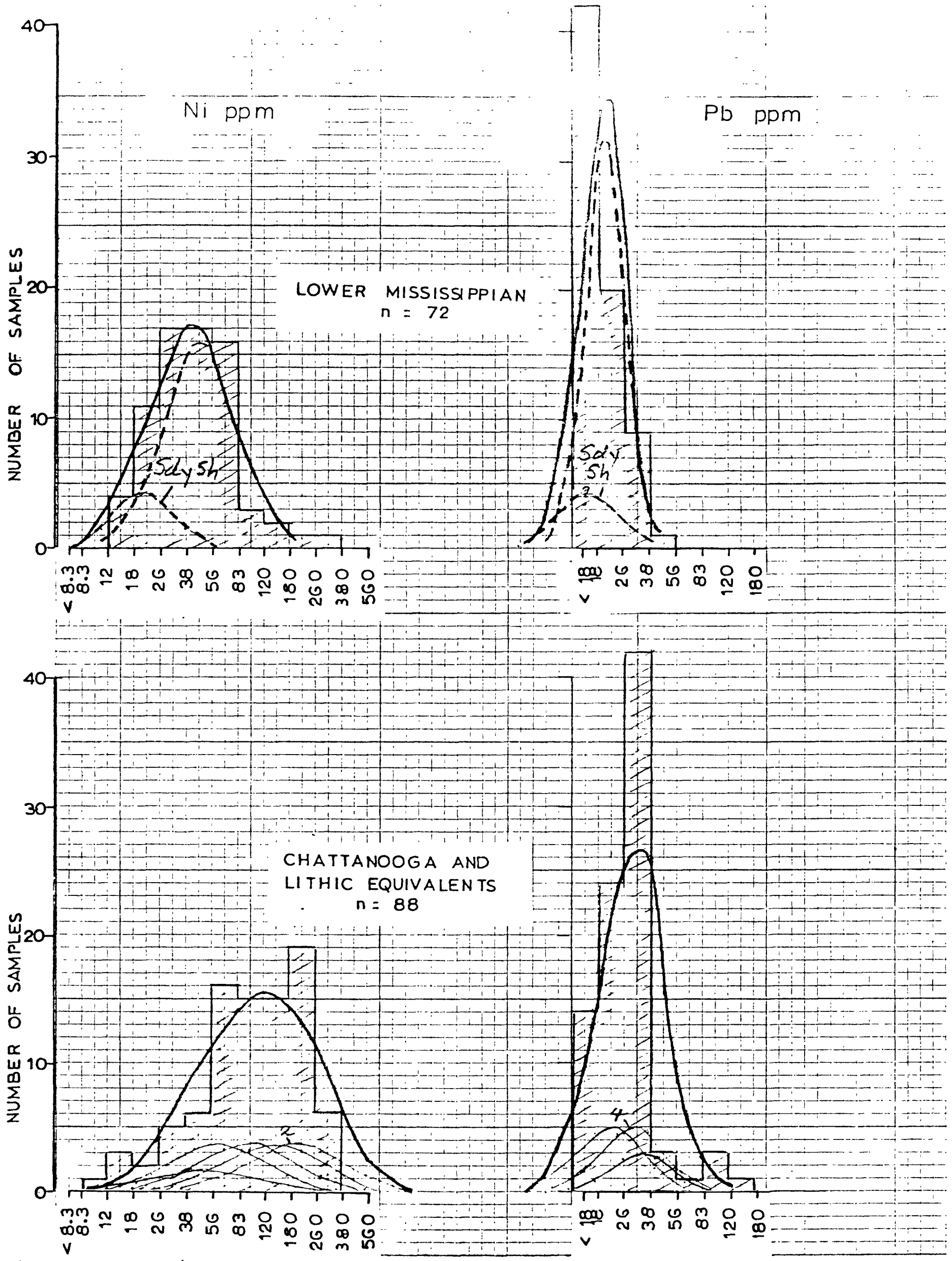



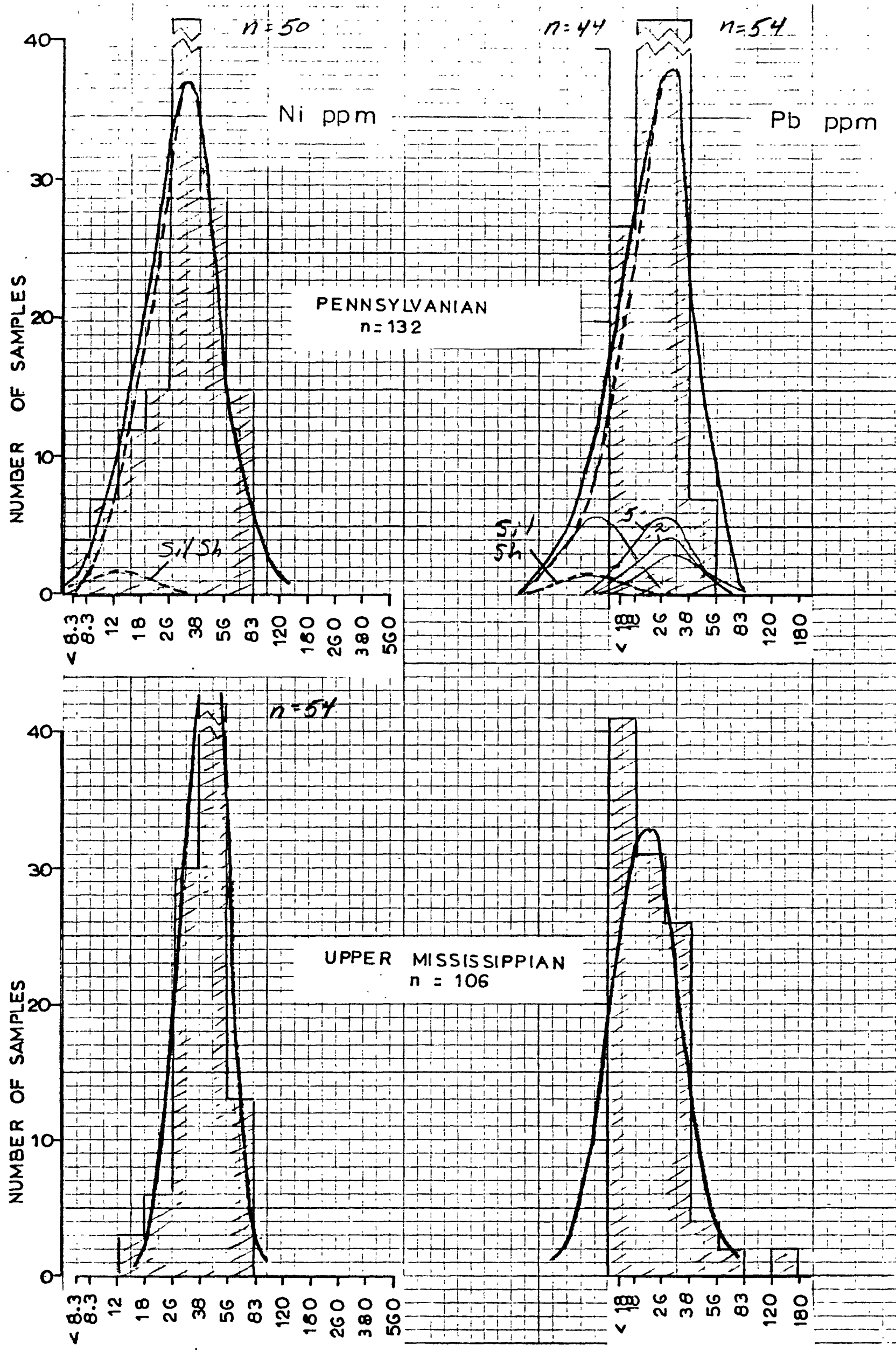

Figure 6.--Continued 








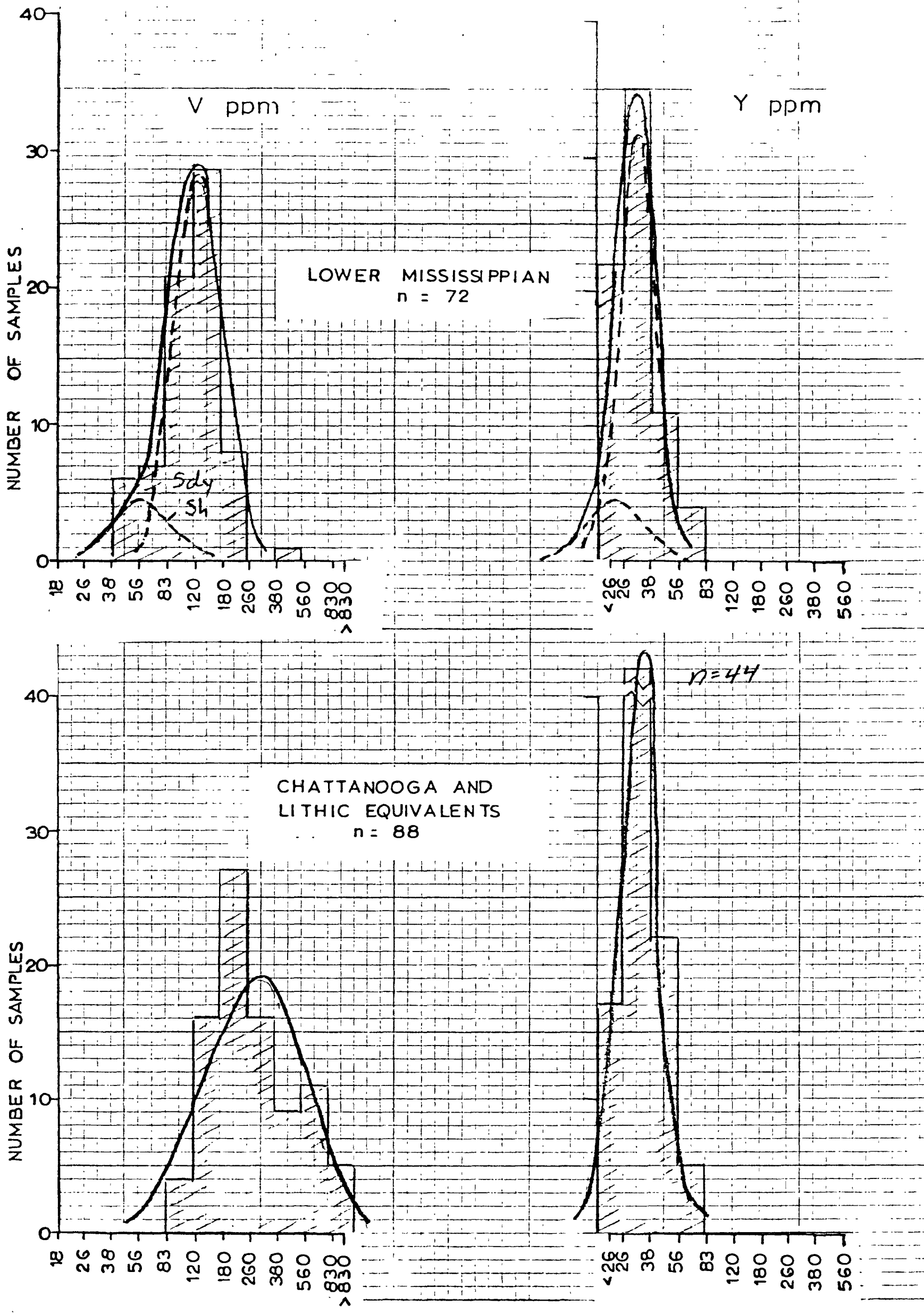

Figure 6.--Continued 


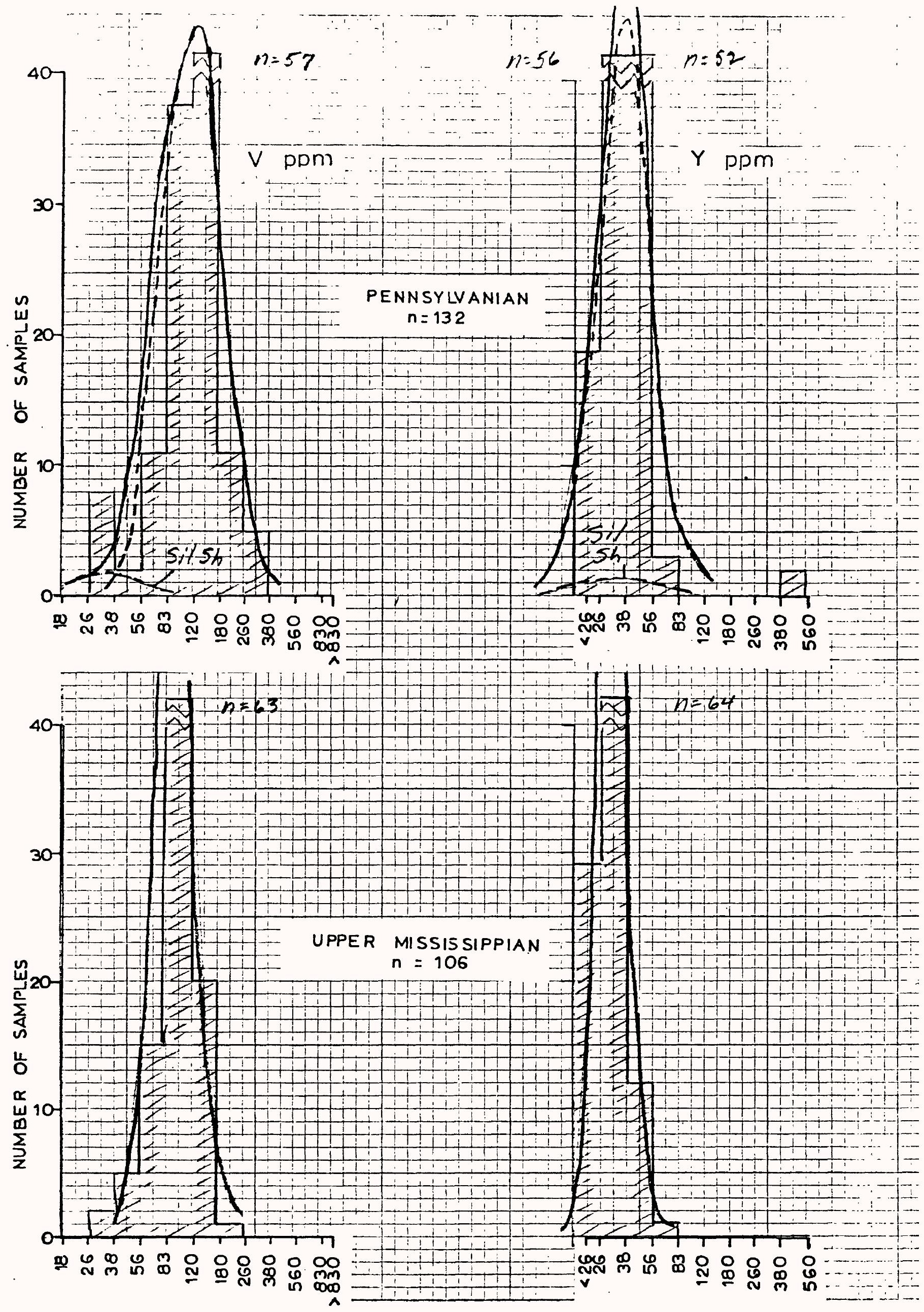




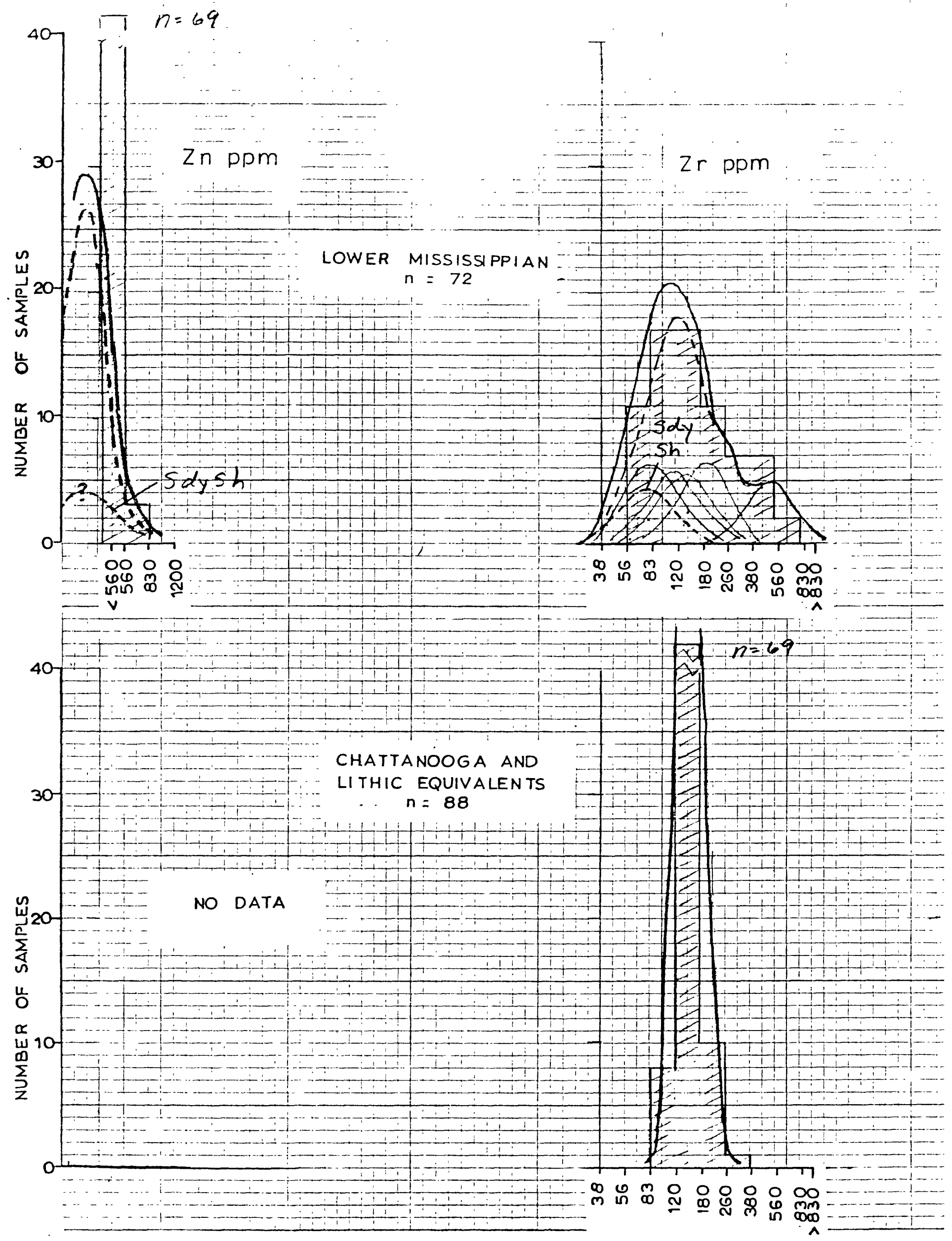




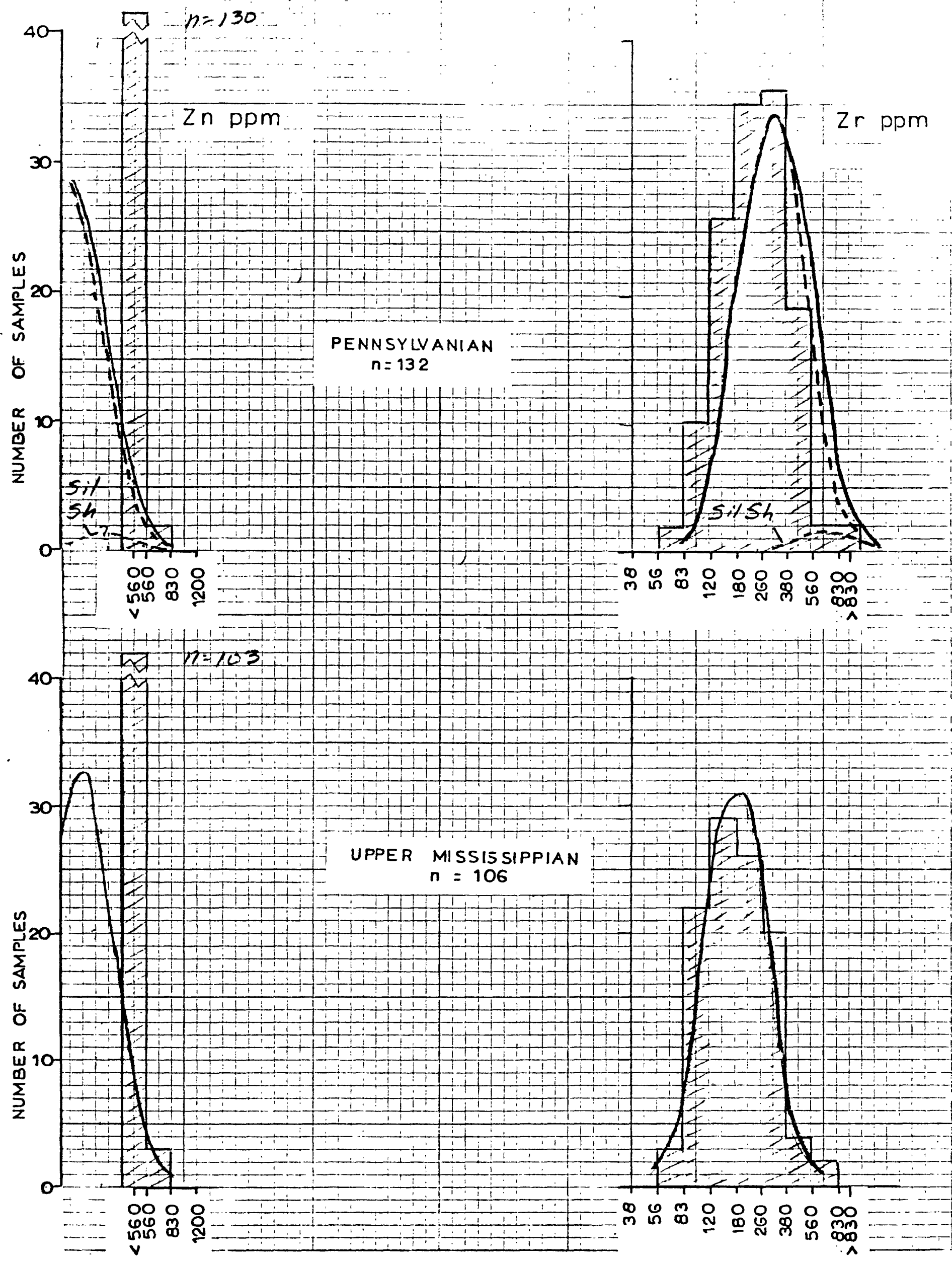

Figure 6.--Continued 
Chaplin, J. R., and Mason, C. E., 1978, Geologic map of the Garrison quadrangle, Kentucky-Ohio, and part of the Pond Run quadrangle, Lewis County, Kentucky: U.S. Geological Survey Map GQ-1490.

Cluff, R. M., and Reinbold, M. L., 1978, Anoxic conditions during New Albany Shale Group (Devonian-Mississippian) deposition in the Illinois Basin (abs): Geological Society of America Abstracts with Programs, v. 10, no. 6, p. 249.

Conant, L. C., and Swanson, V. E., 1961, Chattanooga Shale and related rocks of central Tennessee and nearby areas: U.S. Geological Survey Professional Paper 357, $91 \mathrm{p}$.

Connor, J. J., 1981, Geochemical analyses and summaries of carbonate rocks from Kentucky: U.S. Geological Survey Open-File Report 81-509, 120 p.

Crider, A. F., 1913, Economic geology of the Tell City and Owensboro quadrangles: Kentucky Geological Survey, Ser. 4, v. 1, p. 263-316.

Erickson, R. L., 1966, Geologic map of part of the Friendship quadrangle: U.S. Geological Survey Map GQ-526.

Ghosh, Birendranath, 1951, Random distances within a rectangle and between two rectangles: Calcutta Mathematical Society Bulletin, v. 43, p. 17-24.

Havens, R. G., and Myers, A. T., 1973, Direct-reader spectrometric analyses in Geochemistry of Lower Eocene Sandstones in the Rocky Moutain Region: U. $\overline{\text { S. }}$ Geological Survey Professional Paper 789, p. 13.

Horne, J. C., Ferm, J. C., and Swinchatt, J. P., 1974, Depositional model for the Mississippian-Pennsylvanian boundary in northeastern Kentucky: Geological Society of America Special Paper 148, p. 97, 114.

Kepferle, R. C., 1966, Geologic map of the Elizabethtown quadrangle, Hardin and Larue Counties, Kentucky: U.S. Geological Survey Map GQ-559.

Kepferle, R. C., and Lewis, R. Q., Sr., 1974, Knifely Sandstone and Cane Valley Limestone--Two new members of the Fort Payne Formation (Lower Mississippian) in south-central Kentucky in Changes in stratigraphic nomenclature by the U.S. Geological Survey, 1972: U.S. Geological Survey Bulletin 1394-A, p. A63-A70.

Lambert, T. W., and MacCary, L. M., 1964, Geology of the Briensburg quadrangle, Kentucky: U.S. Geological Survey Geologic Quadrangle Map GQ-327.

Leventhal, J. S., 1978, Sources of organic matter in Devonian black shales (abs): Geological Society of America Abstracts with Programs, v. 10, no. 7, p. 444.

McKee, E. D., and Crosby, E. J., coordinators, 1975, Paleotectonic investigations of the Pennsylvanian system in the United States: U.S. 
Geological Survey Professional Paper 853, 192 p.

Miesch, A. T., 1976, Sampling designs for geochemical surveys--Syllabus for a short course: U.S. Geological Survey Open-File Report 76-772, 128 p.

Sable, E. G., 1979, Eastern interior basin region in Paleotectonic investigations of the Mississippian System in the United States, Part I, Introduction and regional analyses of the Mississippian System: U.S. Geological Survey Professional Paper 1010, p. 59-106.

Sable, E. G., Kepferle, R. C., and Peterson, W. L., 1966, Harrodsburg Limestone in Kentucky: U. S. Geological Survey Bulletin 1224-I, p. Il-Il2.

Shapiro, Leonard, and Brannock, W. W., 1962, Rapid analysis of silicate, carbonate, and phosphate rocks: U.S. Geological Survey Bulletin 1144-A, 56 p.

Siever, Raymond, 1951, The Mississippian-Pennsylvanian unconformity in southern Illinois: American Association of Petroleum Geologists Bulletin, v. 35, no. 3, p. 542-581.

Swann, D. H., 1964, Late Mississippian rythmic sediments of Mississippi Valley: American. Association of Petroleum Geologists Bulletin, v. 48, no. 5, p. $637-658$.

Thaden, R. E., Lewis, R. Q., Sr., Cattermole, J. M., and Taylor, A. R., 1961, Reefs in the Fort Payne Formation of Mississippian age, south-central Kentucky, in Geological Survey Research 1961: U.S. Geological Survey Professional Paper 424-B, p. B88-B90.

Wanless, H. R., 1975a, Appalachian region in Paleotectonic investigations of the Pennsylvanian System in the United States, Part I, Introduction and regional analyses of the Pennsylvanian System: U.S. Geological Survey Professional Paper 853, p. 17-62.

1975b, Illinois basin region in Paleotectonic investigations of the Pennsylvanian System in the United States, Part I, Introduction and regional analyses of the Pennsylvanian System: U.S. Geological Survey Professional Paper 853, p. 71-96. 
ํㅗำ i.?.?.? is

in

Ona o I MMMnN

i no.0.0.0.0.0.0

i

i is

1 $\sim-m \sim \approx 0 \approx \approx \sim m$

I. 1

E !

an î 0000000000

$\because c$ เ

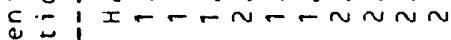

$\sum \stackrel{0}{c}$

$\therefore=$

QE

$\therefore 2$ I ช

$\therefore ن:$

x.=

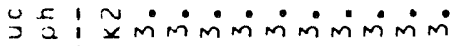
20

c

$¥ 01: 0$

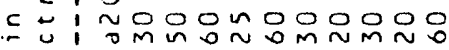
a 1 ( \pm is

년

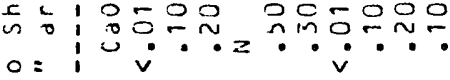

$\therefore$ is ?

$0=1$ iे0000000000

TC

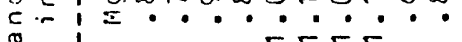

$\times$

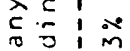

o c

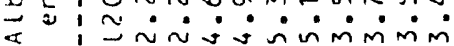

ज)

$3 \stackrel{4}{2}$

-

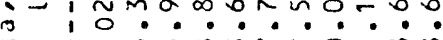

ज|:

$\circ 2$

$\begin{array}{ll:l}c & 0 & 1 \\ 0 & 0 & 1\end{array}$

Ho inomona omnm

이료

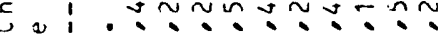

-

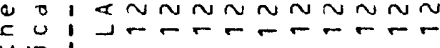

o

t. I i 00000000000

응응ㅇㅇㅇㅇㅇㅇㅇㅇㅇㅇㅇㅇ

«ิ

¿1

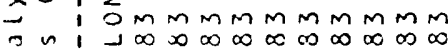

c

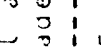

2 1 20000000030

$u \sim 1$ 工

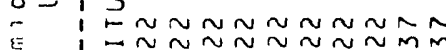

LU:

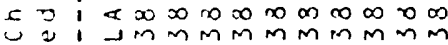

1,0

i.

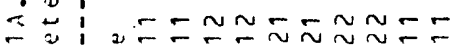

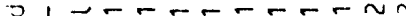

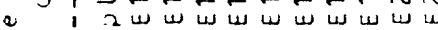

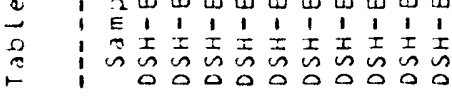

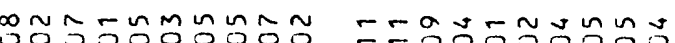

..0.0.0.0.0.

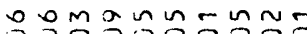

ว.?.:.?.:?.:0

.......?

$\sim a-\infty \infty \infty-r m \sim$

$00 \div 00 . \div \div \div \simeq$

$\because 0 \div 9$ ํํํํำ

onn nonorño

อำำำํำ

ñ

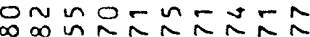

-.?

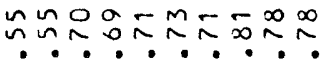

mon

n๐:

-・・

0000000000

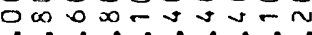
$n-n n \pi n+\infty$

-000000000

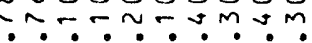
$\because \because \div \div \div \div$

0000000000

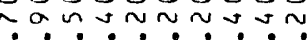
ran

OnOOO

$\because 20.0$ $\therefore \sim$

no $00 m-m \infty-a$ जि-rijinim

$\sim n \infty \infty a \sigma \infty m 00$

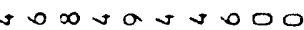
iñ $\dot{\sim} \dot{\sim} \dot{0} \sim \dot{0}$

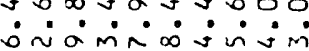

$\sim \infty \sim \infty 0$ $\dot{0} \dot{0} \dot{0}:$

- In U vamaan

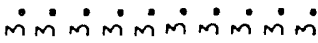

nooratan a n

n-antamon-o

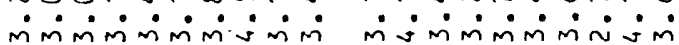

o o o o n

minini

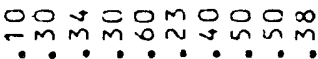

은은ํํํำ

oonoonoodo oิ $\therefore \because \div \div \div$

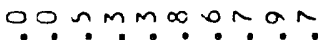
نंmininimin $\ldots-\ldots \ldots$

$\sim \sim m-m \backsim n \sim-$ $\because \cdot \dot{0} \dot{0} \dot{\sim} \dot{n}$ $\tilde{0}-\infty$ s

$0 \cos ^{2}-\infty 0-\infty$ $\infty \infty-m 0-0 \infty 0-$

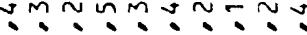

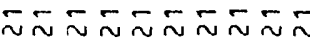

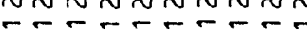

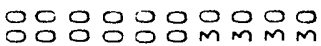

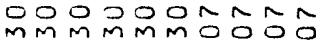
mmm $\infty \infty \infty \infty \infty \infty \infty \infty \infty$

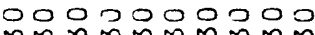
$\hat{M} \hat{m} \hat{m} \hat{m} \hat{m} \hat{m} \hat{O} \hat{O} \hat{O}$ $\infty \omega \infty \infty \infty \sim \sim \sim N$ MMMMMMnMMM

$\simeq \approx-\tilde{\sim} \approx=-\simeq \simeq$

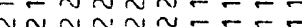
w山 w w w w

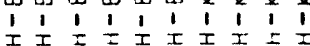

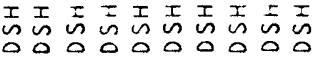

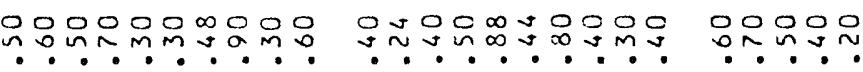

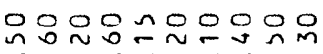

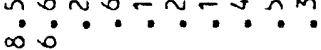

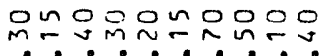

oo noo

$\because \div \frac{0}{1} \div$

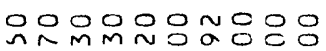

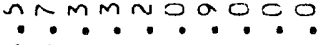

0000000000 ㄴ? ㄴ.?

잉ㅇㅇㅇ $\because 9 . \div \div$

$\sim \infty 0 \sim \infty \infty \cup m \Omega$ in

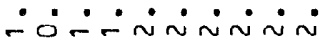

$m-\infty-\sim 00 \sim 00$ نं $\dot{1} \dot{\sim} \dot{0} \dot{0} \dot{0}$

a O OM N $\therefore \div 0 \div$

$\sim \infty \infty \sim n \backsim \sim n-a$

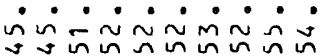

$0-\infty \infty \sim \sim 0 v \operatorname{sun}$ $\dot{0} \dot{\hat{n}} \dot{\sim} \dot{\sim} \dot{\sim} \dot{\sim} \tilde{n}$

movom in in in

$n-n \sim m-\backsim m \sim n$ mamorn

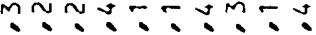

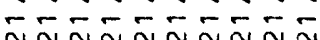
nan- $-\cdots$

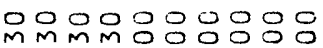

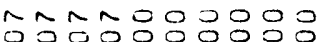

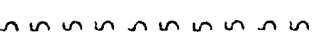
$\infty \infty \infty \infty \infty \infty \infty \infty \infty$

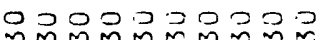

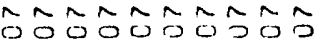
ヘヘヘヘヘヘヘヘヘヘ

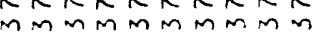

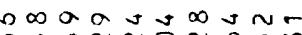
$m \sim \tilde{N} \approx 0 \approx 0 \backsim m$

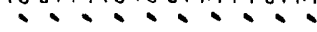

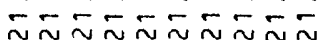
$\cdots+\cdots+\cdots$

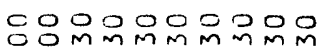

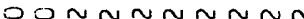
OO 0 m n m n n

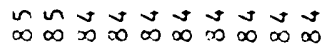

$m \infty u n$

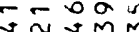
$\because \because \because M m$ $\bar{\sim} \bar{\sim} \bar{n}$

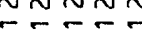

M응요 $\sim \sim \sim \sim \sim$ $\sim \sim \sim \sim \sim$ $\vec{\infty} \sim \vec{\infty} \vec{\infty} \vec{\infty}$

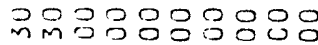

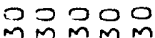
층영ㅇㅇㅇㅇㅇㅇㅇㅇㅇ

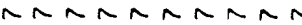
MMMMMMMMMM

$\tilde{\sim} \sim \tilde{\sim} \tilde{\sim}$ $\sim \sim N N N$ MMMMM

$\bar{\sim} \approx \approx \Xi=\because \approx \bar{\sim}$ $\bar{x} \bar{x} \bar{x} \tilde{x} \tilde{x} \tilde{x}$ $\begin{array}{llllllllll}1 & 1 & 1 & 1 & 1 & 1 & 1 & 1 & 1 & 1\end{array}$

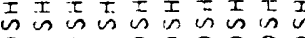
م00000000 $\tilde{\sim} \approx \Xi \Xi \simeq \simeq \Sigma \bar{\sim} \tilde{\sim}$

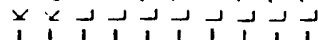

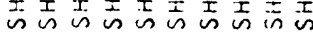

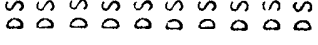

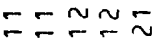
$\overrightarrow{1} \overrightarrow{1} \overrightarrow{1} \overrightarrow{1}$

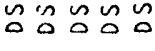
$\bar{\sim} \check{\sim} \approx$ $\sim \sim--\ldots-\ldots \approx=$ IIIII 


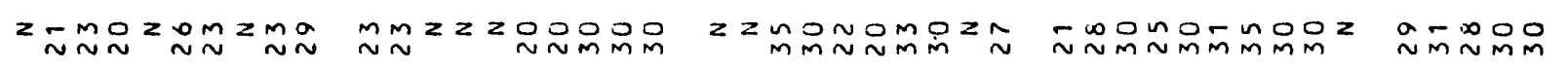

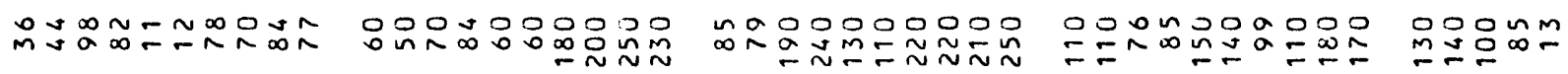

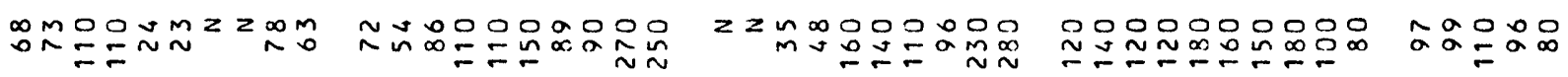

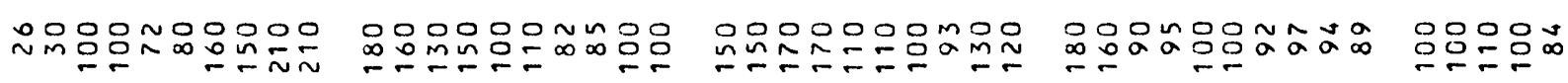

ñmกnnoo oลำ $-\sim$

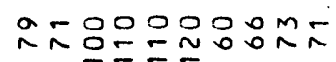

$z z=0 z z a z \tilde{N}$

$a z z z z$ MิMNa

응ㅇㅁ은은유운옹

moñuñum

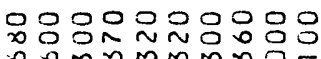

omnmmin:

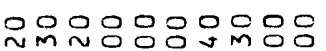

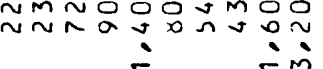

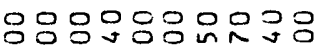

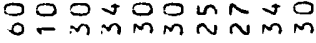
ì
응잉ㅇㅇㅇ mNㅡ는

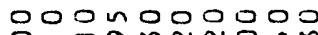

OF

in

i

a

is

boom o d o d n m mmonmstan

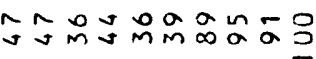

은웅응으은은으은

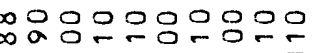

0000000000

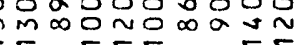

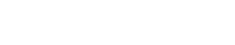

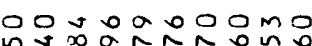

央范

ปที่ง

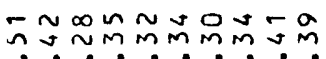

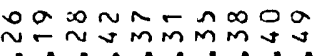

J-

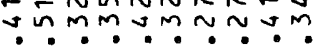

in $0 N 0 m$

ษ.

moง?ำ-

$\because \therefore \dot{n} \dot{\sim} \dot{\sigma} \dot{\sim} \dot{m}$

$\dot{m} \dot{\sim} \dot{\sim}-\dot{\sim} \dot{0} \dot{0} \dot{0}$

$\sim \infty \% \sim \infty ? m m ?$

vono omonom iिi் $\dot{\sim} \dot{m} \dot{\sim} \dot{\sim} \dot{v}$

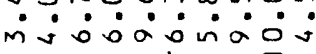

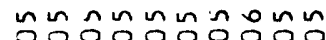
o000000000 $\dot{v} \ddot{v}_{v} \dot{v} \dot{v} \dot{v}_{v}$

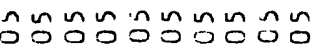
$\dot{v} \dot{v} \cdot \dot{v} \dot{v} \dot{v} \cdot$

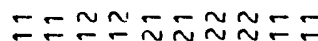

$\sim N-\sigma \approx \sim-r \sim \sim$

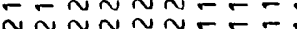
$\tilde{w} \widetilde{\sim} \sim \widetilde{w} \widetilde{w} \bar{x}$

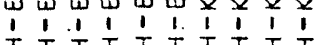

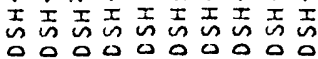

공ำ

vNopoctoos

$\dot{\alpha} \dot{v} \dot{v} \dot{v} \dot{v} \dot{v} \dot{v}$

$-\approx-\approx \bar{\sim} \bar{\sim} \approx \sim \sim$

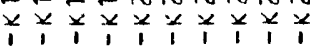

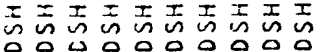

$\bar{\sim} \approx \approx \Xi E \simeq \approx \bar{n}$

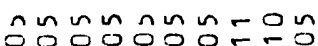

$0000000=-0$ $\dot{v} \dot{v} \dot{v} \dot{v} \dot{v} \cdot \dot{v}_{v}$

$\approx \approx E=\approx \approx \Sigma \bar{\approx} \approx \cong$ $\approx \approx-E \div \approx N \approx N=$

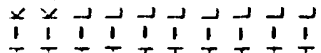

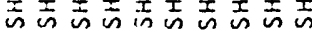

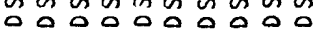

mำำำ

․ำ.

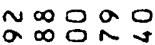
눈

ํํำ的车

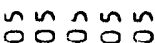
viviv

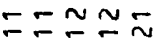
$\check{\sim} \tilde{\sim} \sim \sim ⿻$ । $\overrightarrow{1} \overrightarrow{1} \overrightarrow{1}$

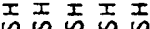

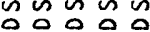




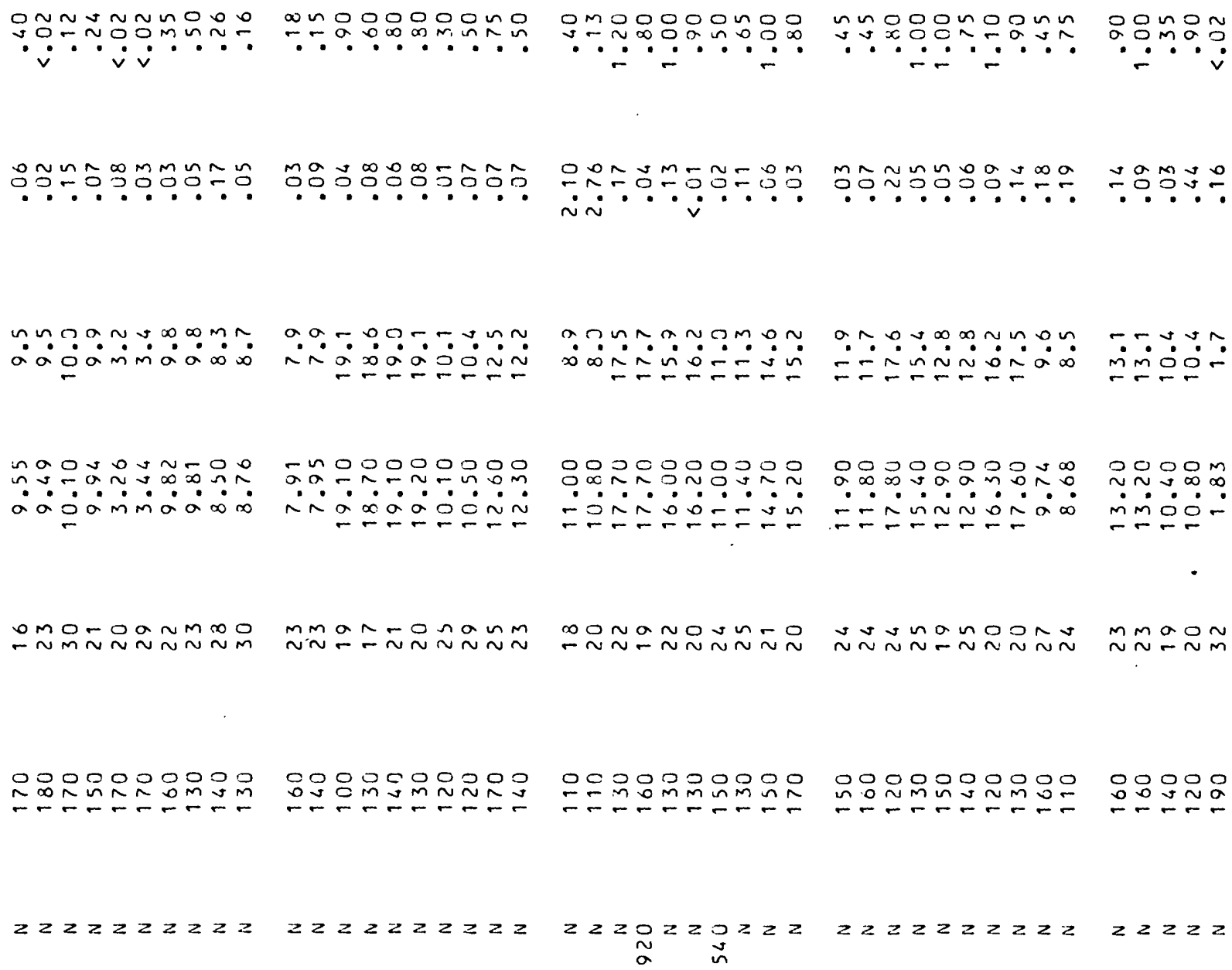

nn $\rightarrow, x, 0,5,0, \pi)$

으으은 …?... ?.?.?

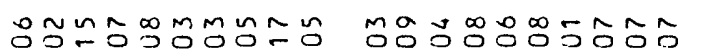

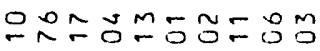

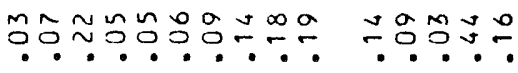

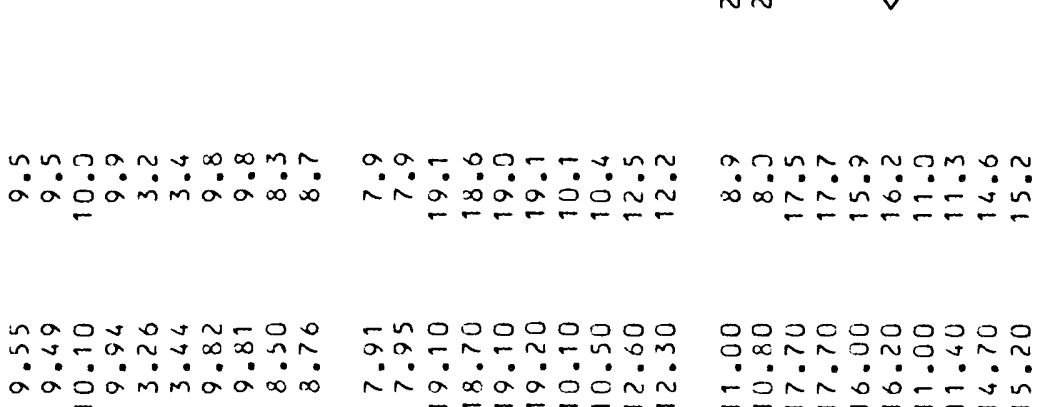

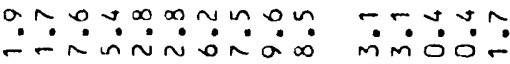

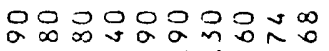
$\because \dot{\sim} \dot{\sim} \sim \dot{\sim} \dot{\sim} \dot{\sim} \dot{0}$

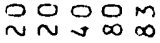
míं0:

OMO-OONM⿻O

mman-onanm

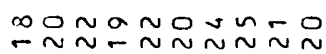

ปัก

mmaON MNT-NกNNNN

0000000000 noññ

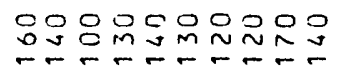

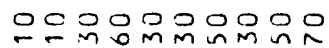

잉ㅇㅇㅇㅇㅇㅇㅇㅇㅇㅇ응

$0 ㅇ ㅇ ㅇ ㅇ ㅇ$ 그느느는

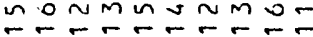

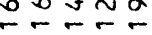

ก̃̃

MMN소

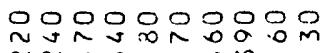

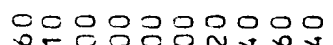
กับ

$\therefore \div \div \div$

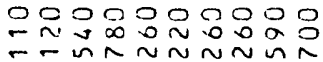

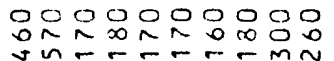

$\begin{array}{lll}0 & 0 & 0 \\ 0 & 0 & 0 \\ -1 & m\end{array}$

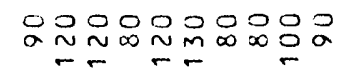

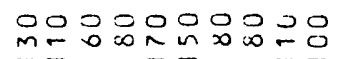

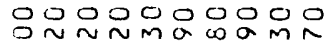

0000000000

$\begin{array}{rl}0.0000 & 0 \\ 0 & 0\end{array}$

mกoณnaกนง

$\because n \approx a \simeq \backsim n-n$ ก

ñonom nn O O

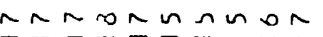

$m \backsim \sim m o$

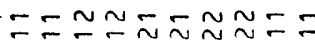

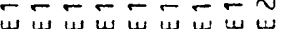

111111111

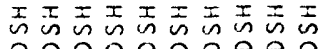

0000000000

$\approx \approx \Xi=\simeq \cong \check{\sim} \sim$

⿵冂丶

1 $1 \frac{1}{1} 1 \frac{1}{2} \frac{1}{1} 1$

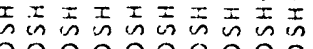

$=\mp \pm \sim \pi$ บבบ 1111 仝杢志杰 


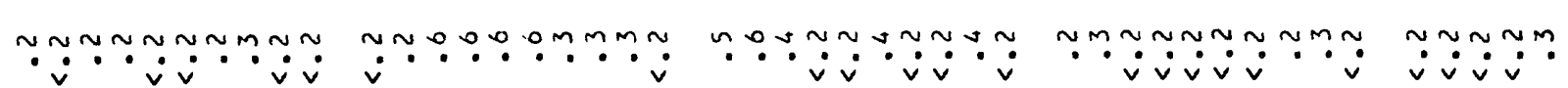

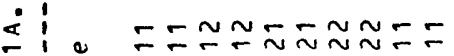

$\simeq \simeq \bar{\sim} \tilde{\sim} \tilde{\sim}= \pm \simeq \sim$

$\check{\sim} \approx \tilde{N}= \pm \sim \sim \bar{\sim}$

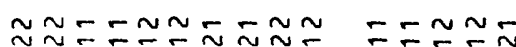

2

$\sim \sim \sim \sim \sim \sim---\bar{x}----\sim N \sim \sim \sim \sim$

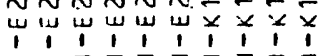

$\bar{x} \bar{x} \bar{x} \bar{x} \tilde{N} \tilde{\sim} \tilde{x} \tilde{x} \tilde{x}$

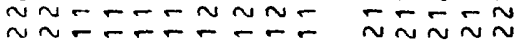

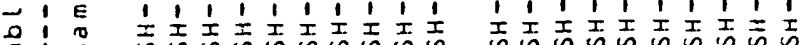



ํํํํํํํํํํำำ

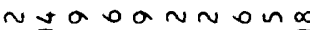

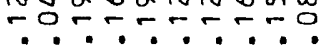

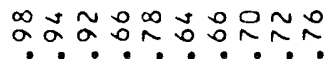

OODOONmOOON กับ? r N N r

ì

!

- $a$ onn $\sim N \sim m a$

$\bar{\sim} \approx \tilde{N}=\bar{\sim} \approx \bar{\sim} \tilde{\sim}$ $\simeq \sim \sim-\frac{\alpha}{\alpha}-\frac{\pi}{\alpha}-\frac{\pi}{\alpha}$

ก ก แก ก ก a a a

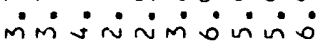

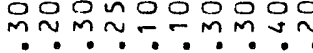

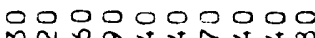
ก……………

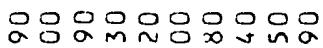
$\because \because \sim \sim \dot{\sim} \dot{\sim} \therefore$

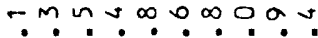

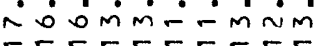

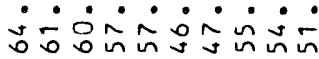
$m-m \sim-\ldots \sim \sim m \sim$ $\therefore \therefore: \div: \div:$

$\sim \sim \sim N \sim \sim \sim \sim \sim \sim ⿻ 上 丨$

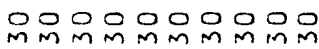

$\approx \approx \approx \hat{O} \hat{O} \tilde{\partial} \hat{O} \tilde{O}$

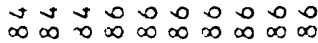

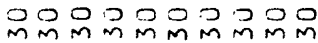

$\approx \approx \approx N \tilde{M} M \hat{M} \hat{M} \bar{M}$

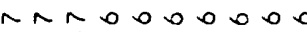

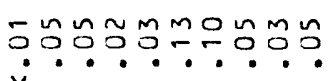

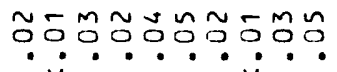

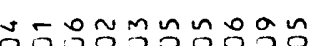

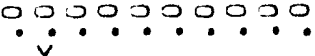

งัดก

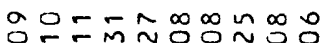

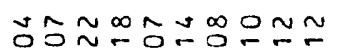

mํํㅇํํํํํํำ

ํํㅇำ

왱ㅇㅇㅇㅇㅇㅇㅇㅛ

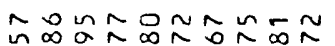

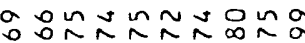

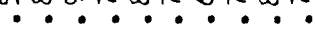

-.?

aำ

-

$\because \infty \% \div \infty$ ?

$0000700 \pi 00$

$\infty \sim 00 \% m 0 \infty \wedge m$

$\therefore \div \div \div$

0000000000

$\lambda \sim \sim u g m m-m \infty$

$0 \infty 0$

$m \infty 0$

$\because \div$

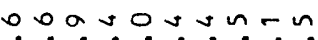

oan an n m o o

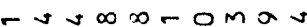

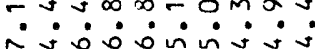

$\because \because \because$

o on numummm

$\sim \sim a \infty m \sim v \backsim 0 \infty$

o o a $\infty$ mor $\sim \infty$

$-\sim \sim$ نmiminivij:

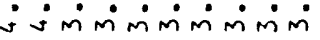

minimisं $\dot{0} \dot{0}$

$\because \sim \sim$

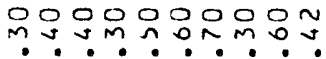

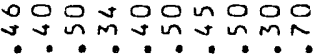

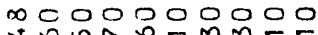

으음음

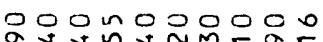

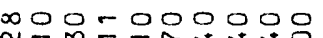

. . . . . n m

ก.บ.

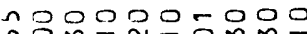

กับกัดmM- กำ

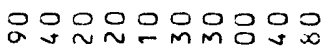

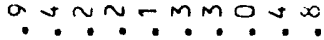

Noomoooooo

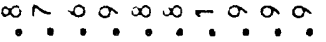

0000090000

ก 00 a $0--\infty \sim$

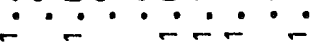

$ㅇ ㅣ ㅇ ㅛ$

- .

$\because 0 \% \div \infty m \sim n m$ $\dot{m} \dot{\sim} \dot{\sim} \dot{\sim} \dot{\sim} \dot{\sim} \dot{\mathrm{j}}$

$\infty \infty \infty \sim \cdots-\sim a m-$ نim $\dot{\sim} \dot{\sim} \dot{\sim} \dot{\sim} \dot{\sim}$

a. 0.0 0. $00 \%$

- $\sim \dot{\sim} \dot{\sim} \dot{\sim} \dot{0} \dot{0} \dot{0}$

$\because \div$ man

- $n$ 는

$a \simeq \simeq$

$\checkmark v 00$ ง vnmm nmo-mnoOn

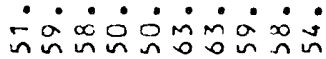

ก- OM NOONO-

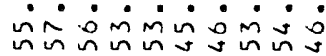

תinouar n toa

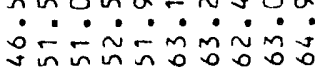

0,00

-mmñ NOO $n m$ กำ

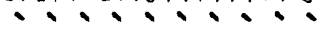

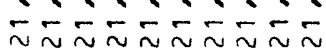

$\because \simeq-\ldots \sim \simeq \simeq \simeq \simeq$

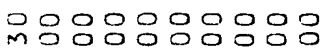
등ㅇㅇㅇㅇㅇㅇㅇㅇㅇㅇㅇㅛ

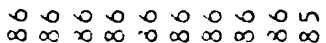

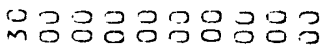
$\hat{m} \mathfrak{y}$ in $n$ in $n$ in $n$ in

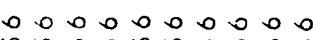

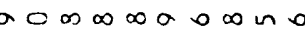
UOO !. . . . ! ! ! - $\approx \approx \Sigma \bar{\sim} \approx \bar{\sim}$

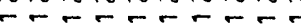

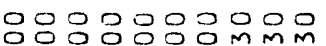

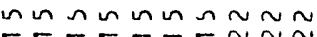

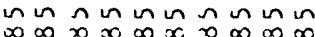

tonomañon $\checkmark 000, \infty \infty \approx 0 m$ $\because m \simeq 5 \div \div \div \div 5$

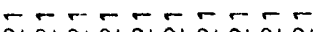
$\bar{\sim} \check{\sim} \bar{\sim} \bar{\sim} \check{\sim}$

-

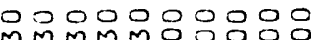
$\approx \approx \sim \sim \approx 09000$ $\Omega \ln \ln \Omega \infty \infty \infty \infty \infty$ $\infty \infty \infty \infty \infty \infty \infty \infty \infty \infty$

in $\infty$

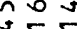
$\therefore \div \div$ $\bar{\sim} \bar{\sim}$

용요 $\because \sim n$ $\infty \infty \infty$

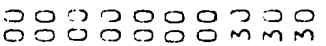

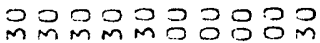
을음

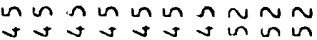

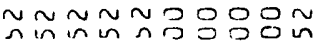
in $\mathcal{N} \tilde{n}$ MMMMMM O O O O M

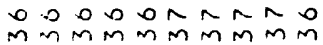
inm

$\approx \Xi=\approx \sim \bar{\sim} \approx \approx \Sigma$ $\sim \sim \sim \sim \sim \sim \sim \sim \sim F$

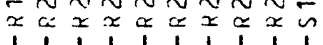

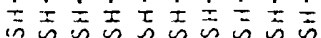

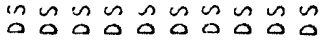

$=\simeq \approx \bar{\sim} \approx \approx \Xi E \simeq$ $1111111\}$

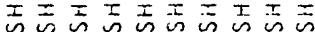

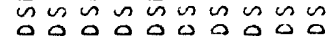
$\overline{5}=-\bar{n}=\approx \tilde{n}$ $\simeq \Sigma \bar{\sim} \approx \Sigma=\approx \sim=$ $\sim \sim \sim \sim \sim-\overline{0} \approx \sim$

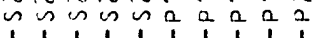

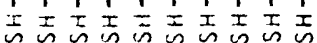

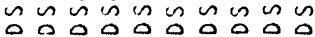

$-\sim \sim$

$\bar{\sim} \bar{\sim}$ 20 a $\begin{array}{lll}1 & 1 & 1 \\ 1 & 1 & 1\end{array}$ 另吉杰 응 


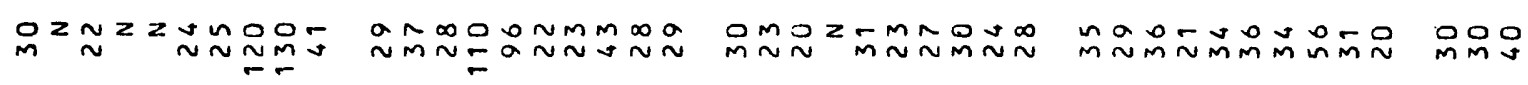

mogooo0000 0000000000

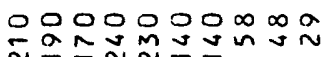

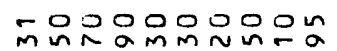

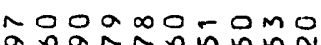

$20 \Omega$

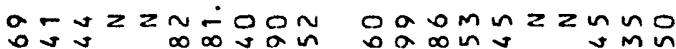

oogoonmarz

$2 \ln _{\sim}^{\infty}$

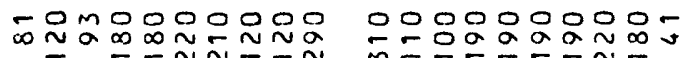

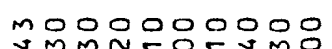

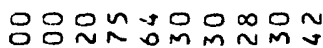

ํํํํํำ

క

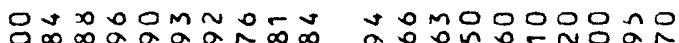

NNNEOENM은

-r

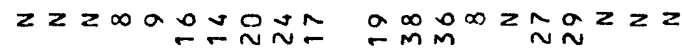

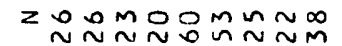

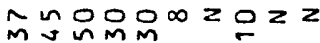

20요

$\operatorname{lom} 0$

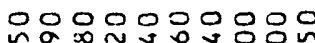

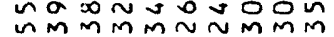

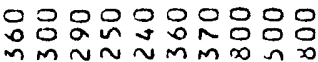

in:

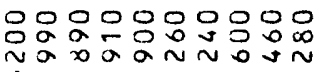
$\sim$

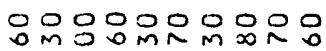

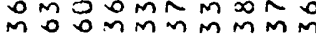

응요 $\stackrel{\sim}{\sim} \tilde{N}$

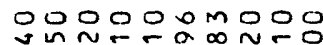

ปேะะะ

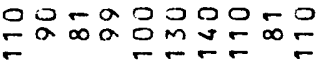

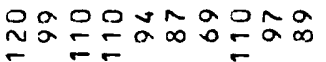

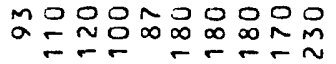

엇음음 $\approx \simeq m$

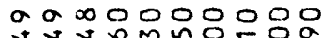
ำn

은유응ㅇㅁ음

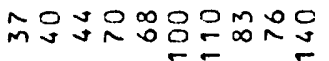

in

Nํํำ

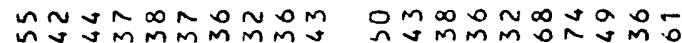

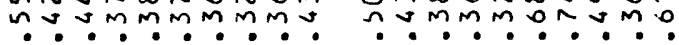

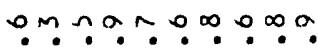

amosinon and oñmis imimó

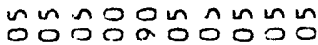
$\dot{v} \dot{v} \dot{\sim} \dot{v} \dot{v} \cdot \dot{v}$

$\bar{\sim} \approx \tilde{N}=\tilde{\sim} \sim \bar{\sim} \tilde{\sim}$

$\sim \sim \sim \bar{\alpha}-\bar{x} \bar{\alpha} \bar{\alpha} \bar{\alpha}$

1111111111

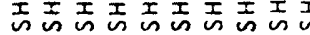

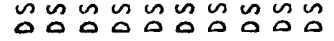

- $\dot{0}$ inmmijir

ก 0.700 .00 .00

$\sim--\sim \sim--\approx \sim \sigma$

$\approx \Xi=\approx \sim \bar{\sim} \approx \approx \Sigma$

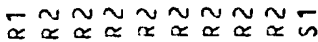
111111111

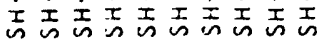

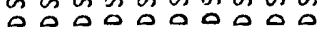

- กิก

$m \sim \infty \infty 000 \infty v N$ m.? mmut? $\circ \approx \tilde{n}$

$\min a \operatorname{singanad}$

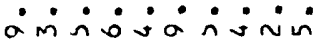

-omanusmon $\ddot{\sim} \dot{\infty} \dot{\operatorname{sim}} \dot{\sim} \dot{\sim} \dot{\sim}$

$a m a$

$\dot{i} \dot{\sim}$

mnn nnn :0.0.0.:0.0.

nกm 0.0.0.00.0.0.

nก ก

$\dot{v} \dot{v}$

$=\approx \sim-\bar{\sim} \approx \approx=\Sigma \simeq$

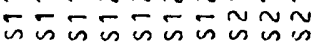
111111111

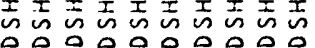

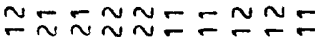
$\approx \approx \approx \approx N=\mp= \pm \bar{N}$

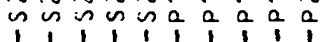
志志志吉贡志志志志

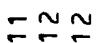
$\bar{\sim} \pi$ a: a 1! นูกั 
$-\sim \infty$ inounoono

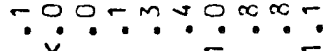

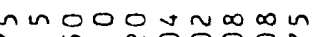

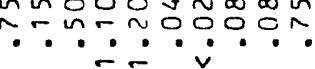

onmogno

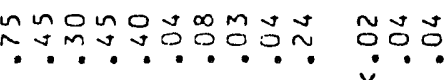

$00=n a-00 \sim \sim$

$\because \because \because n: ?$ ํ.?

ㄴํㅇํำง

$\tilde{0} 0 \simeq \simeq \sim 0 \infty \circ \sim n$

ํํํํํํํํํํํํํํํำ 웅

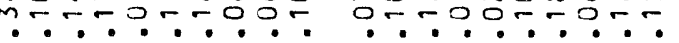

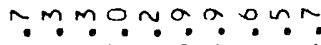

- inisinisi⿺辶

r.n.

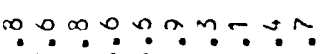

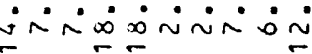

…0.0.0.0.0.0.

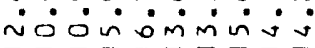

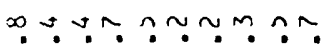

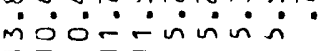

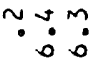

$0 \approx \sim 000,000$

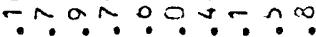

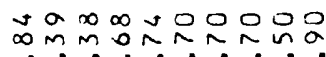

-inisionis

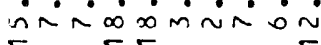

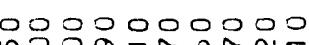

ก․․…ง

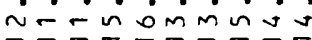

ㅇoㅇoㅇㅁㅇㅢ.

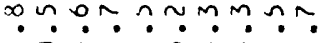
mojóninin

$m: \infty$

moanmoavmo $m \sim \sim \sim N \sim \sim N \sim$

$0-m \sim-\min n a v$

ond onnognu

Dom $\sim \sim \sim \sim \sim \sim \sim N \cong \Omega$

$m \infty 0$

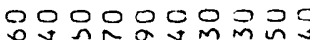

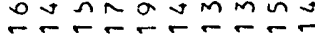

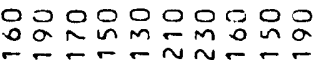

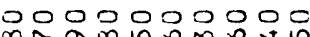

mand

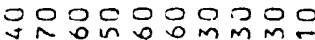

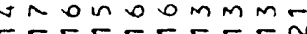

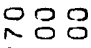

2

is

$z \geq 2 z 2 z 2002$ 8.

$2 z 2 z 2 z 2 z 2 z$

$2 z 2 z 2 z 2 z 2 z$

$z z \geq z z z z z$

$2 z 2$

OM $m \sim n m \infty 0 \infty$

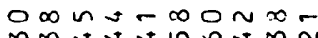

แnกOONOMN

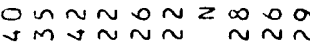

은수

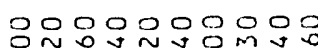

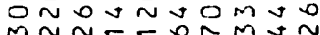

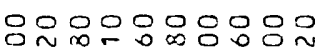

0000000이응

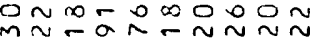

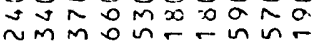

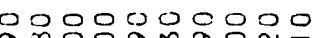
a

이응 $\approx \approx m$

0000000000

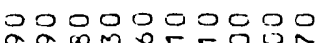

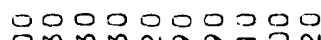

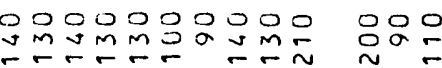
m=ㅇaㅇำ - $\sim N$ I N

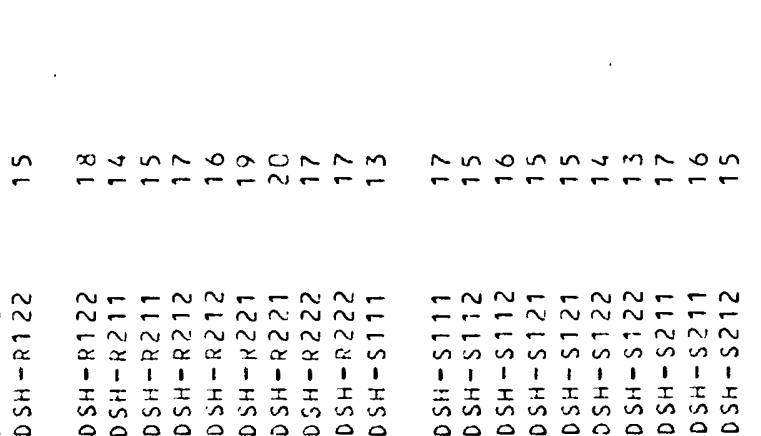

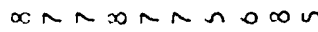

aramand

rathunhnom

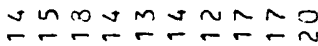

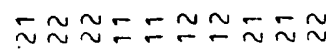
곰 $\tilde{x} \bar{x} \bar{x} \bar{x} \bar{x}$

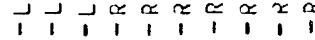
志志至志志志志

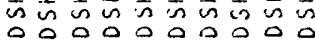

ำ

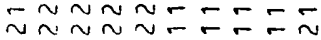

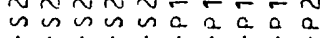

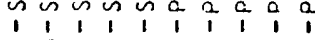

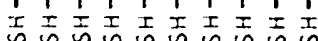

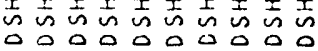

$\approx \bar{\Sigma} \approx \approx \sigma=-\sim \sim-$ $-\sim \sim$

$\bar{\sim} \bar{\sim}$

$a$ a a

II

is 


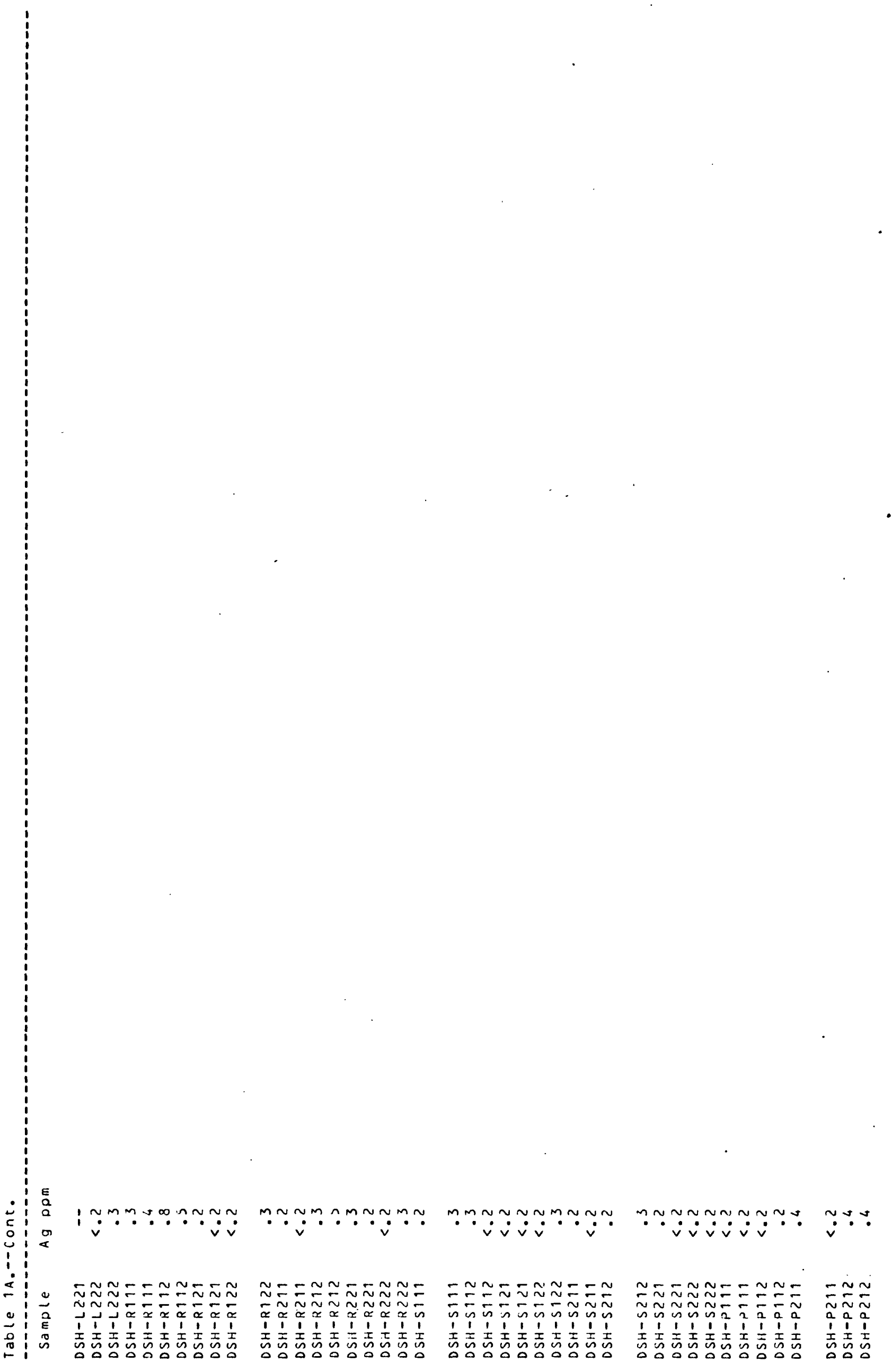


$\sim \sim \sim a \sim n v \sim-\sim 0$ oa 0 a เ・・・・・・・ i.

ion i I i

$\stackrel{2}{+\infty}$

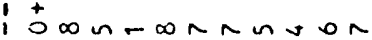
Í $\dot{\sim} \dot{\sim} \dot{\sim} \dot{m} \dot{\sim} \dot{-}$

is

:̊̊n

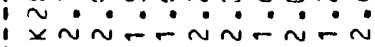
i

Öoㅇoㅇoㄴ

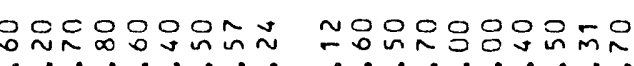
$a$ $\therefore c:$

$\because$ c :

č I 药 ¿ .5 !

Q E :

$\therefore \stackrel{0}{2}$ : $00000000 \sim 0$

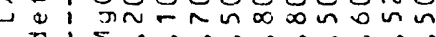

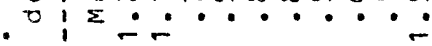
安

J.

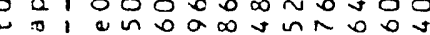

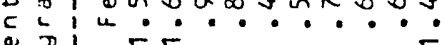
$\times$ ㄴ

$\therefore$ :

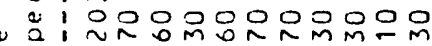
on umini $\dot{\sim} \dot{m} \dot{m} \dot{\sim} \dot{j}$ $\frac{1}{c} c_{0}^{\infty}$

$z=0$

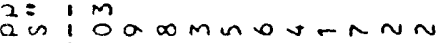

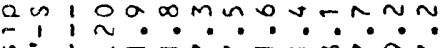

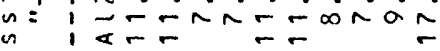
in $\therefore$;

$\simeq \pi$ :

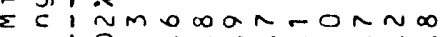
-

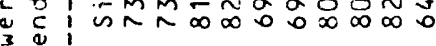

$\stackrel{\circ}{a}$

-

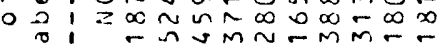

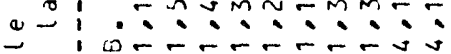

r..

乐: 象

t I 00000000000

ㄱ 30090000000

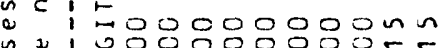
n

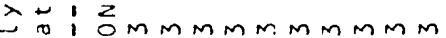
$001, \infty \infty_{\infty} \infty \infty_{\infty} \infty \infty_{\infty} \infty \infty_{\infty}$ ro

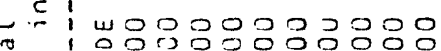

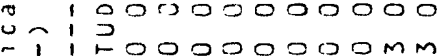

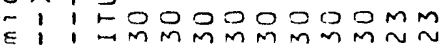

E $1: 5 M M M M M M n M N \sim$

En

is

?

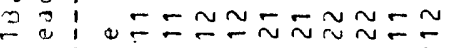

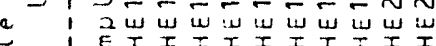

D

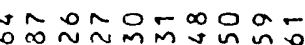

$\because \because n m \cup n \sim \infty$

กกตกับ00000

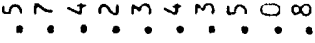

$\checkmark 00-000 \cong \because m$

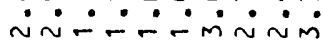

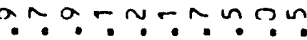

$\because \because \because \because \dot{\sim} \dot{m}$

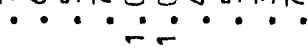

옹ㅇㅇㅇㅇㅇ으응 $0 \ln a 0-\infty a m b$

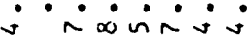

in $\pi, \infty$ a o in $\infty$ - inminim- -

$\checkmark 0 \pi 0000000$

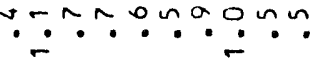
언응응ㅇㅇㅇㅇㅇㅇㅇㅇㅇ음 мंм- -

- $-\pi$

man $00 a \sim v m$

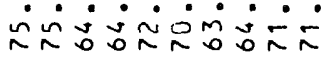

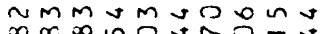

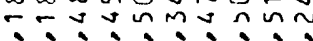

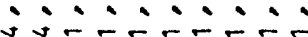
$\stackrel{\sim}{\sim} \bar{\sim} \bar{\sim} \bar{\sim} \bar{\sim} \bar{\sim}$

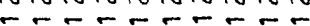

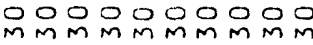

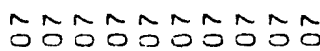
mm $n \cap n \sim n n \sim n n$ $\infty \infty \infty \infty \infty \infty \infty \infty \infty$

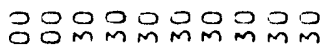
mmnก ก ก ก N nกDOJ00000

$\infty \infty \sim \sim N \sim \sim \sim N$ N $M M M M M M M M M M$

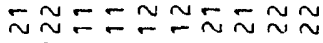
w N $\bar{x} \bar{x} \bar{x} \bar{x} \bar{x}$

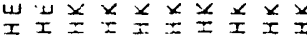

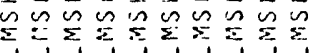

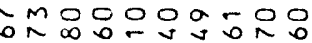

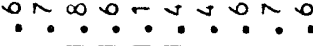

กงก๊์

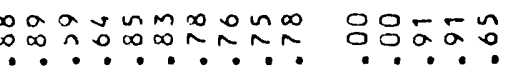

$--n-$

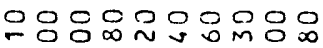

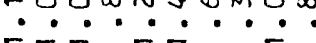

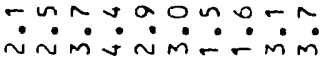

$\because m \sim \sim ง \infty \div m a \div$

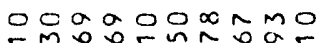

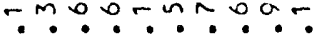

응ㅇㅇㅇㅇㅛ

บํ.?

$\because \because \infty r \div \sim n 0 \sim 9$ $\sim \sim m \cup \sim \sim \dot{m}$

0000000000 $\because m \sim \simeq \sim m \div \div \backsim m$

$-\infty \sim 0 n s \infty \infty 0 \infty$ róvininiómi

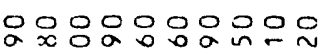
- $\dot{i} \dot{-} \dot{-} \dot{i} \dot{\sim}$

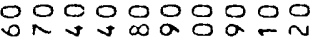
……?

ind 0 $\therefore \therefore \therefore-$

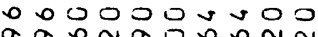
- iñí í

0000000000 ma $a_{0}$ volun $\because \because \therefore \dot{\sim} \dot{\therefore} \div$

00000 ง

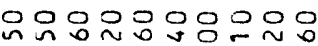

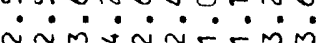

앙응ㅇㅁ영응은은

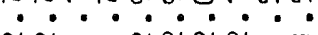

응응ㅇㅇ음 мंजिंмं

mon áñ⿻三丨寸

$00-\infty n m \backsim n m v$

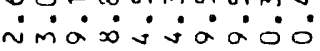

$\min 00$ nar

ก ง $a \alpha \infty-m-0$

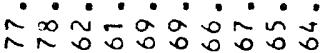

$\because \neg m \cap \div-\infty m \cap \infty$

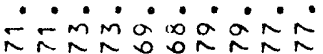

-

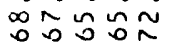

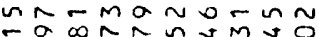

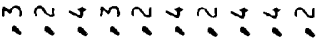
$\because \therefore \because \therefore \therefore \div \div$ $\bar{\sim} \bar{\sim} \bar{\sim} \bar{\sim} \bar{\sim} \bar{\sim} \bar{\sim}$

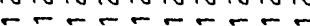

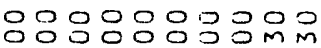
잉ㅇㅇㅇㅇㅇㅇㅛ

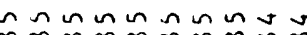

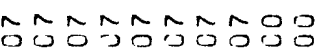

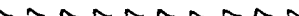
MnNMMMnMMM

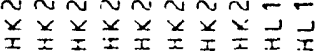

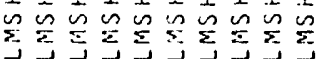

on on $0 \mathrm{~m}$ n a m ono Oñ

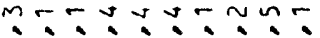
$\bar{\sim} \check{\sim} \bar{\sim} \check{\sim} \check{\sim}$

in nood

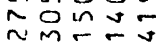
$\because \therefore \therefore \div$ $\bar{\sim} \bar{\sim} \check{\sim}$

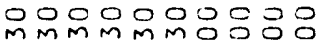
$\sim \sim \sim \sim \sim \sim \sim n \sim n$ in $\cos$ in

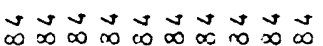

00000 nn n n m $\circlearrowleft \stackrel{\infty}{\infty} \stackrel{\sim}{\infty}$

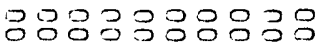
응응으

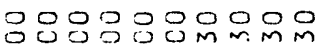
요욤ํ ヘヘNヘヘNヘヘヘn $\sim N \cap N O$ जMMMMMMMMM

$\simeq \approx \bar{\sim} \approx \tilde{\sim}= \pm \sim \sim$ $5=5-5 \simeq \sim \sim 0$ $\vec{I} \vec{I} \vec{I} \vec{I} \vec{I} \vec{I} \vec{I} \vec{I} \vec{I}$

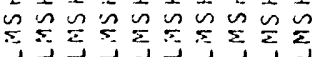

$\sim \approx \approx \approx=$ $\simeq \simeq \widetilde{a}$ III $\Sigma \cong \cong \Sigma$

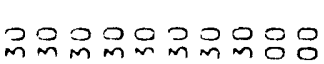

$\Xi=\cong \approx \bar{\approx} \approx \approx \Xi \Xi$ 


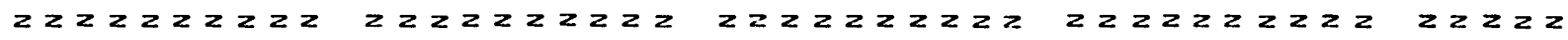

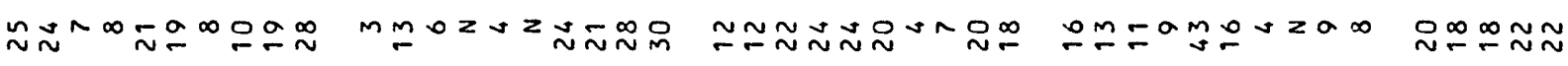

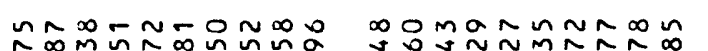

obno-aonkn

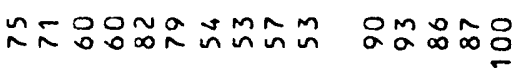

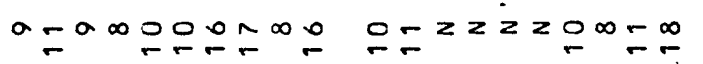

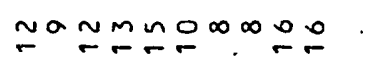

$\because 2 \infty \geq \cong n \geq 2<\infty$

$\because \sim m \sim \sim$

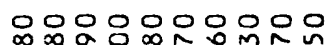

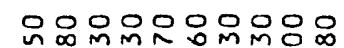

웅응요임용유유

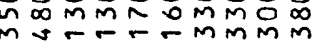
mmo is

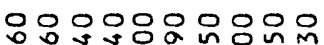

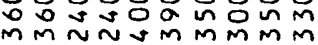

웅웅유음 $\checkmark$ ज mmmmmmu murarmmmi mmo!

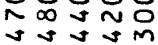

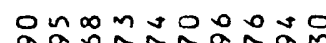
gaonararam

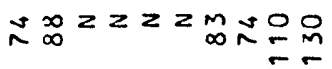

응융ㅇㅁㅇㅢ

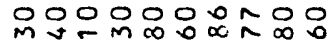

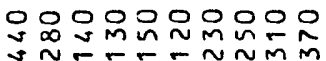

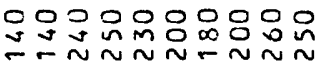

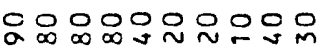

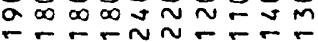

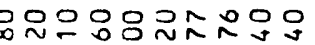
ninuninoon.

은영영유운유음

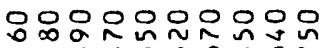

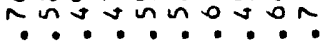
$\because \because \because \frac{1}{4} \div$

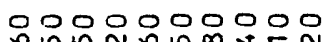
$\dot{0} \dot{\text { insionmin }}$

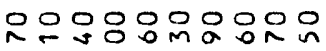

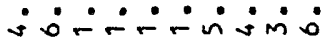

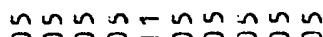
$\dot{v} \dot{v} \cdot \dot{v} \cdot \dot{v} \dot{v} \dot{v}$

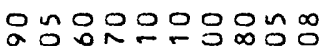

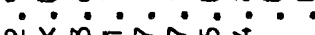
نंที่:

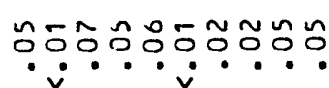

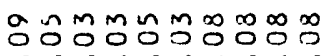

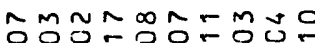

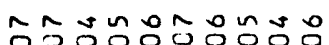

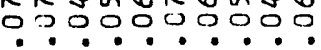

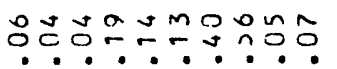

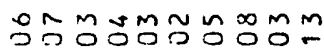

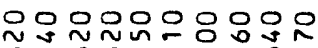
…ำ.

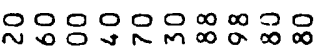

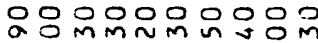
niminorimio: sminino ins

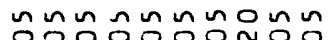
$\dot{v} \dot{v} \dot{v} \dot{v} \dot{i} \dot{v}$

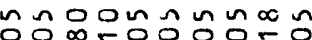
$\dot{v} \dot{v} \dot{\sim} \boldsymbol{v} \dot{v} \dot{v} \dot{v} \cdot \dot{v}$

กูกนกูก

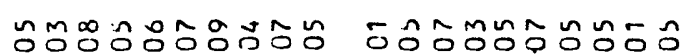
…ํ..ํ..……

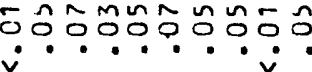

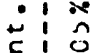

บ:

i!

il $\quad r-a n-r a n-a$

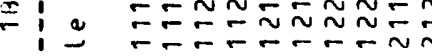

。

0 : 0

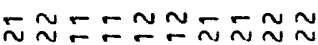
$\sim \approx F=\mp \div \approx N \approx N$

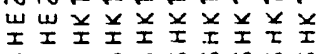
$\sum \sum \sum \sum \sum \sum \sum \Sigma \sum \sum$
$\Xi \Xi \simeq \approx \check{\sim} \approx \approx \Xi \Xi$ $\sim \sim \sim \sim \sim \sim \sim \sim \sim-$

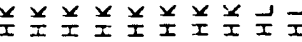

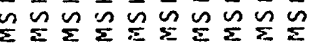

$\simeq \pm \check{\sim} \approx \tilde{N}= \pm \approx \sim$ $-F-\pi=\sim \sim \sim \sim$

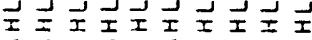

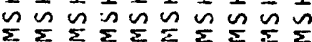

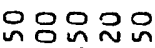
ํำำ.

앵잉ㅇㅇㅇㅇ - $\dot{v} \dot{v} \dot{v}$

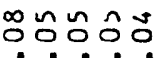

웃ㅇㅇㅇㅇㅇ음 is in

momm: 0.0.0.

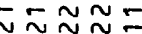
$\sim \sim \sim \sim \pi$

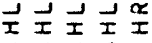
$\sum \sum \sum \sum \sum \sum \sum$ 


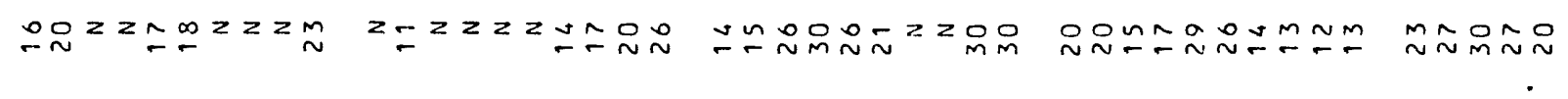

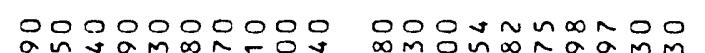

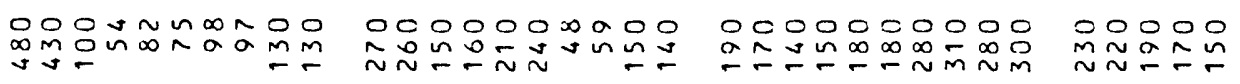

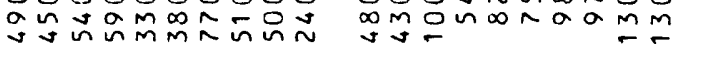

-

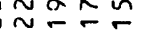

$z z z z z z z z z z z z z z z 20$

$z z z z z z z z z$

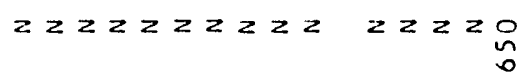

Doa onm no on

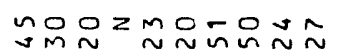

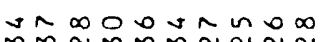

mo응으는

mMaO

(n)

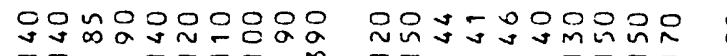

$800000 n \infty 00$

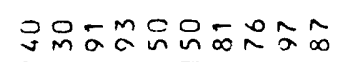

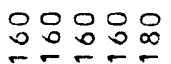

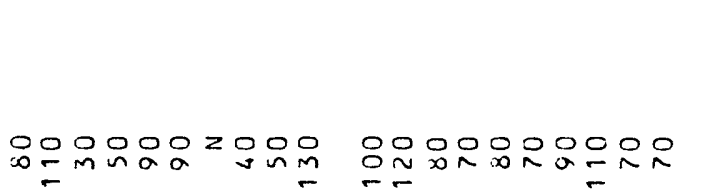
- 
Nㅜㄴmำ

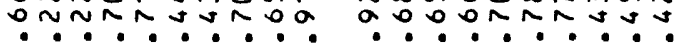

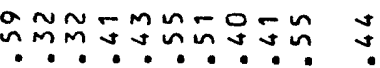

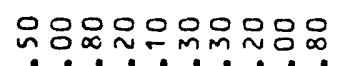

$\therefore \therefore \dot{\sim} \dot{\sim} \dot{m} \dot{m}$

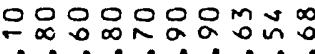

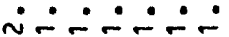

vmuaamva o

$\sim \infty 0000 N m-0$

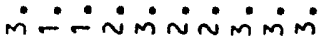

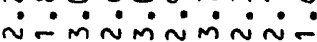

$\because \simeq 0 \sim 0, \infty 0 \infty 0$ $\because \because \because \dot{\sim} \sim \dot{\sim} \ddot{-} \dot{\sim}$

$0-m-\infty 0 \div \sim n \infty$

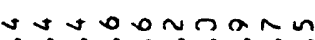

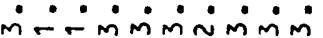

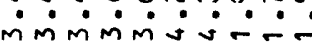

ooogomoono

응ㅇㅇㅇㅇ음은

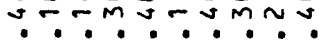

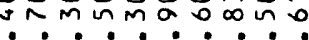

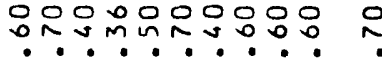

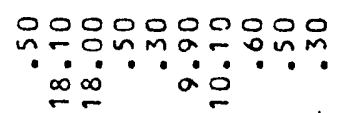

응웃응은응은응응

min

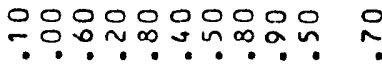
$\dot{m} \approx \dot{\sigma} \dot{0} \dot{0} \dot{0} \dot{0} \dot{\sim}$

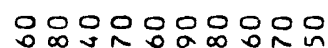

$\therefore \dot{0} \therefore \sim \dot{\sim} \therefore$

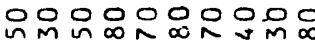

$\because \dot{m} \div \because \because \therefore \div$

o

wo N0000000 onmman-

$\infty \infty a--\infty \infty 0 n$ r $-r$

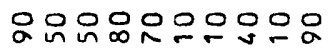
n-ijinisin

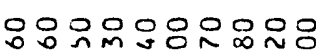

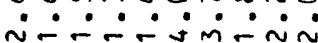

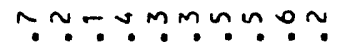

- invináaji

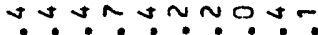

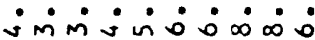

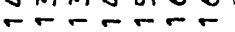

Nma $a \infty \infty m \infty m$ n $\dot{m} \dot{m} \dot{0} \dot{0} \dot{0} \dot{0} \dot{0}$

ma $\infty 0 a-a 0 \infty m$

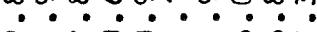

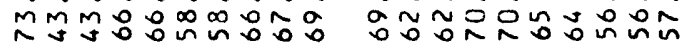

$\infty m n$ o o v OMñ⿻上丨 $\because \div \div \because \because \frac{1}{5}: 5$ $\bar{n} \bar{\sim} \bar{n} \bar{n} \bar{n} \bar{N} \bar{N}$

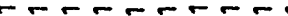

$-0 \infty \infty-r-\infty \infty N$

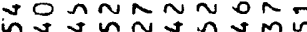
$\because$... $\bar{\sim} \bar{\sim} \bar{\sim} \bar{\sim} \bar{\sim} \bar{\sim} \bar{\sim}$

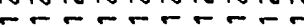

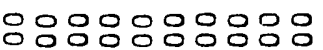

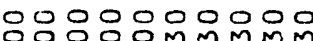

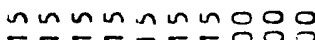

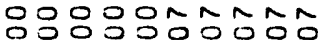

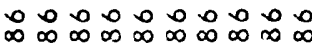
$00000 n n i n n$ $\infty \infty \infty \infty \infty \infty \infty \infty \infty$

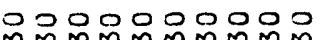

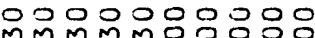
$\hat{M} \tilde{M} \hat{M} \tilde{M} \hat{M} \tilde{M} \bar{M} \hat{M}$ - o o o o o o o D.

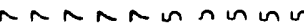
mmmmms

DO 00000000 MnMmMMMMm

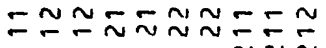
$\bar{x} \bar{x} \bar{x} \bar{x}-\bar{x} \bar{x} \approx \tilde{\approx} \tilde{x}$

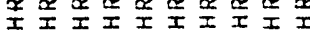
$\sum \sum \sum \sum \sum \sum \sum \sum \sum \sum \sum$

$\sim \Sigma \tilde{\sim} \sim-\sigma \sim \sim \bar{\sim}$ $\sim \sim \sim \sim \sim-F-F$

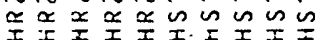
$\sum \sum \sum \sum \sum \sum \sum \sum \sum \sum \sum \sum \sum \sum$

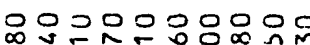
-ㄴ?.?.!.

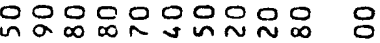

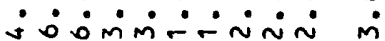

4000000000 $0 \infty \sim \sim 0 \sim \infty \infty \mathrm{m}$ $\because \because \div \dot{\sim} \div$

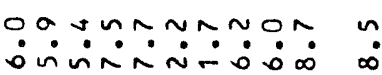

A.

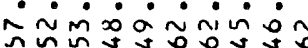

$\checkmark$ mos $x$ nomonn $\hat{m} \sim m \sim n m \backsim \sim n$ $\because$ m

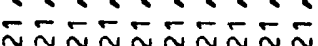
nannan

있인ㅇㅇㅇㅇㅇㅇㅇㅇㅇㅇㅇㅇㅇ응

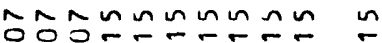

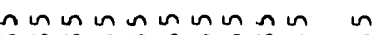
$\infty \propto \infty \infty \infty \infty \infty \infty \infty \infty \infty$

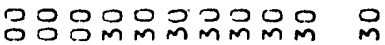

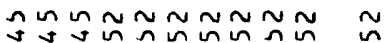
o o o o o o 0.000

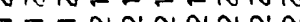

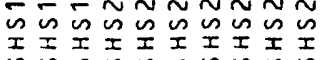

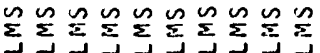

$\bar{\sim} \tilde{\sim} \Sigma=\sim \sim \bar{\sim} \tilde{\sim}$ 


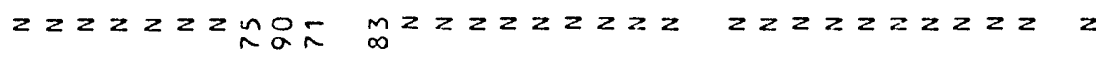

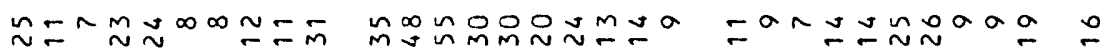

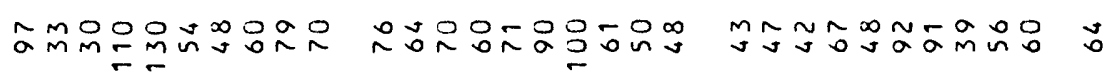

ํำ

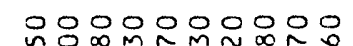

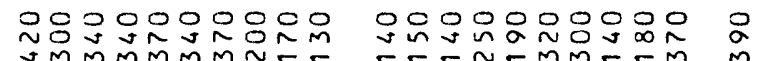

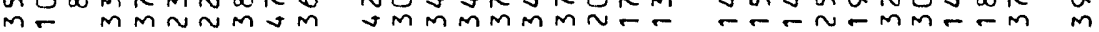

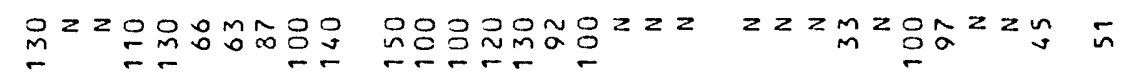

응응요웅응용

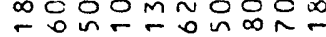

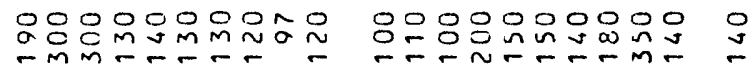
$\therefore$ -

0
$\infty$
$m 00$
00

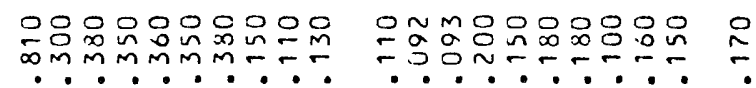

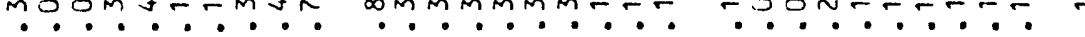

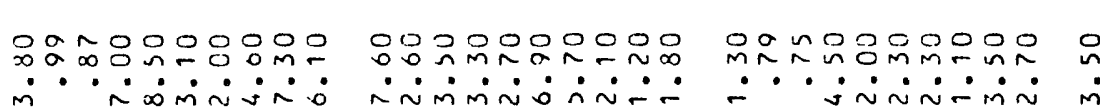

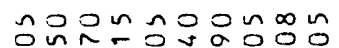

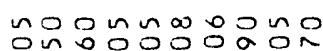

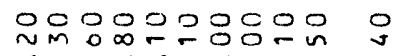

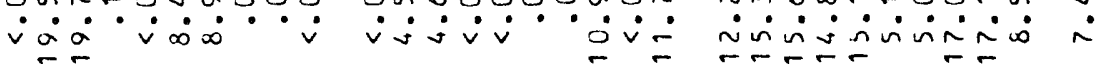

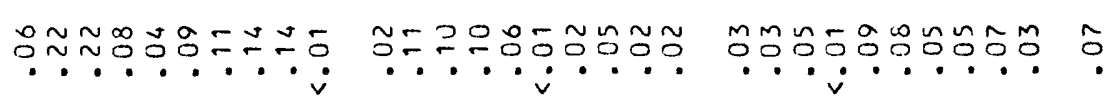

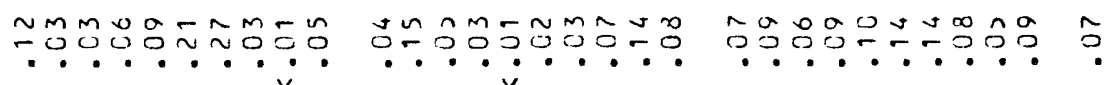

c:

i:

$\Xi \approx \sim \bar{\sim} \approx \tilde{\sim}= \pm \sim$

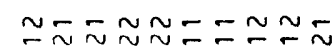

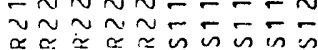

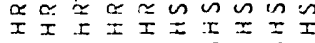

$I=I \equiv I I I I$

$\cong \cong n \Sigma \cong n$

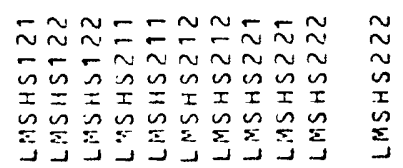




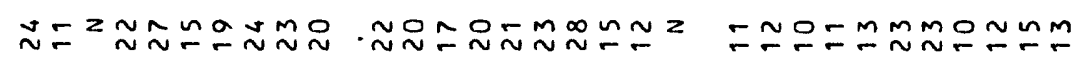

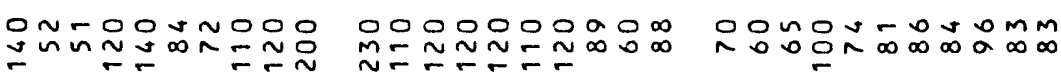

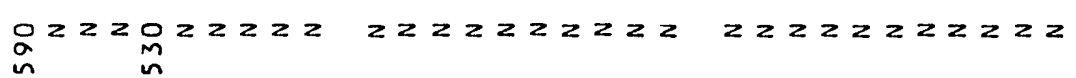

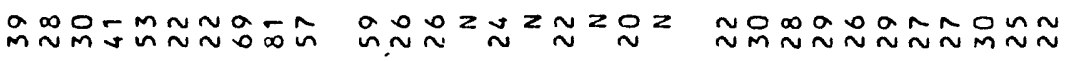

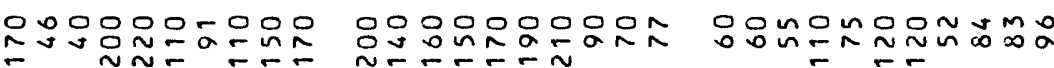

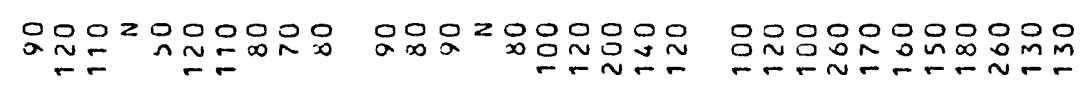

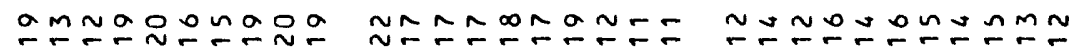
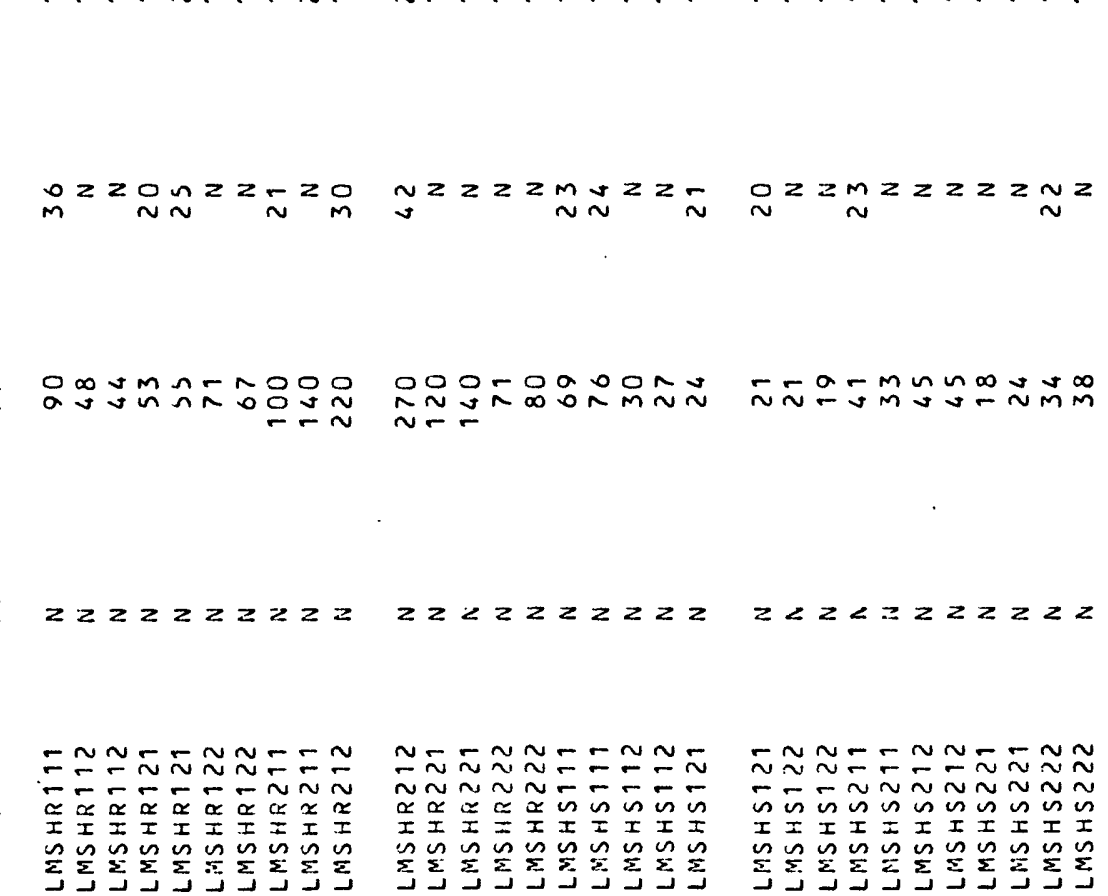

$\simeq \Sigma \Sigma \approx \sim \Xi E \simeq \simeq \bar{N}$

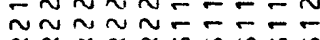
$\alpha \alpha \approx \approx \alpha$ is is is is IIIIIIIII

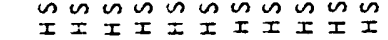

$\bar{\approx} \approx \bar{\Sigma} \approx \simeq \Sigma \bar{\sim} \approx$ $\simeq \sim \sim \approx \tilde{N} \sim \sim \sim \sim ⿻$

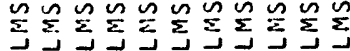


竞

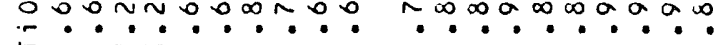

(1)

i.0000000000

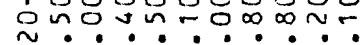

Imisinimisj

i

$\stackrel{+0}{+}$

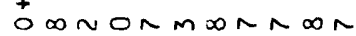

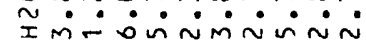

i.

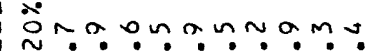

x N N N N OUMm

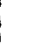

范-

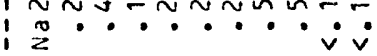
ni

芷它

c. O०0000000000

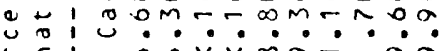

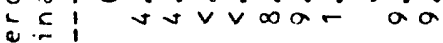

a $E$

$\therefore$ :

U

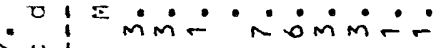

$\begin{array}{l:l}0 . & 0 \\ x & 0\end{array}$

วद

ग

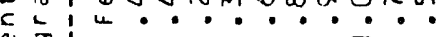

$\times$ ग

c

DaOmnon on $\infty 0$

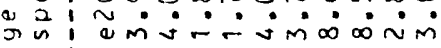

促

$\begin{array}{llll}c & 2 & 1 \\ \pi & 0 & 1\end{array}$

a $=$ ì

an 1 omoonmumnn n! $1 \sim \dot{\sim} \dot{\sim} \dot{\sim} \dot{\sim} \dot{\sim} \dot{m} \dot{m}$ u $<-n \sim N=\mp \infty+\infty$ $\bar{n}=!$

$\simeq$ sis

₹

¿

造 ह

马 $\backsim 1$

- a íorononmma

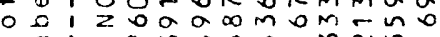

o

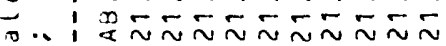

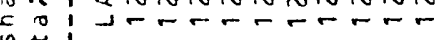
事

$-0$

-

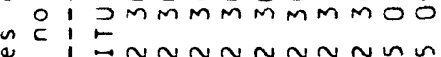

$\backsim$,

$>+$ ¿

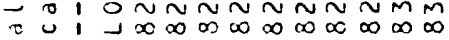

c:

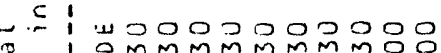

$0-19 M M M M M M M M O O$

E:

EI:HMMMMMMMMMM

- $\quad \varangle \infty \infty \infty \infty \infty \infty \infty N-N$

$\checkmark n 1\lrcorner M M M M M M M M M M$

i)

$\checkmark 0:$ a

- - - - - - - - $N$ N

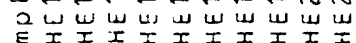

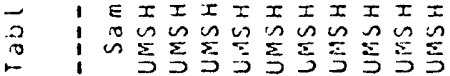

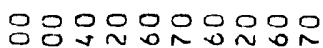
o 0 r miñ $\dot{\sim}-\dot{m} \dot{m} \dot{\sim}$

OOOMM $0000 \mathrm{M}$ мंmimmiñ $\dot{\sim} \dot{m}$

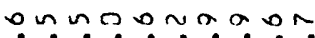
mmnmnna

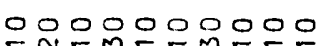
.. .

0000,00000 กำ

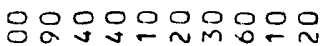
$\dot{\sim} \dot{-1} \dot{-} \dot{-} \dot{-} \dot{-}$

O ก $\because-$

$\sim \sim \sim \infty \sim \sim-a m m$ $\dot{\sim} \sim \dot{\sim} \dot{\sim} \dot{\sim} \dot{m} \dot{m}$

$\infty \sim 0, \infty, \infty, \infty \sim ⿻$ Ḿं $\dot{1}-\dot{0} \dot{0} 0 \dot{0}$ mmamara

$000 \div 0,0 \infty$,

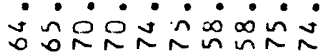

ondo. $a \infty-\infty 0 y \infty n \infty$ t a.

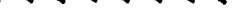

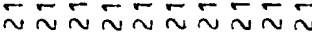

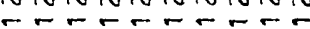

O $000000 \mathrm{O} 0 \mathrm{O}$ nก $n$ un $\operatorname{mon} \sim n$ $-\sigma--L N \sim N N$ $M M M M M M \infty \infty \infty \infty$ $\infty \infty \infty \infty \infty \infty \infty \infty \infty \infty$

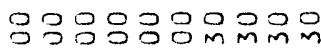

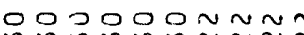
$\sim \sim \sim \sim \sim \sim \sim$ $\sim \sim \sim \sim \sim \sim \sim \sim \sim N$ $M M M M M M M M M$

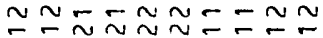
น

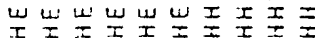

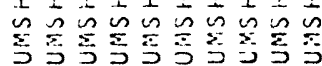

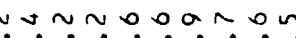

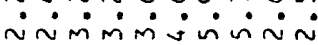

$\ln n \infty v \operatorname{nn} \infty \sim \infty$ $\dot{\sim} \dot{\sim} \dot{\sim} \dot{m} \dot{\sim} \dot{\sim} \dot{\sim}$

inno in $\sin$

$\sim \sim \sim-\approx N \approx-0$

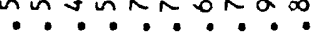

$m \sim \sim m \infty n-j m$ $\sim \sim \sim \sim \%$ ?

$-\approx 0 \sim r$

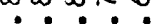

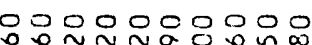
نं $\dot{\sim} \dot{\sim} \dot{\sim} \dot{m} \dot{m}$

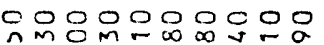

00000 míj $\dot{m} \dot{\sim} \dot{\sim} \dot{\sim} \dot{\sim}$ i் $\dot{0} \dot{0} \dot{-}$

$\infty \infty \backsim 00, \infty \infty$ v

$\because 0 \sim \simeq \infty \sim \infty=\infty$

$\because \div \% ?$

$\because \backsim \infty m \sim m \backsim \sim \div$ ñm ving in

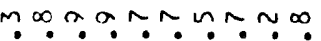
$00 \div \simeq \sim$ $m \sim m M N$

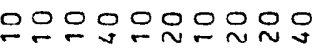

0000000000 man $-\sim \sim 0$ in in $\infty \dot{\infty} \div \dot{\sim} \div \dot{ }$

0000000000

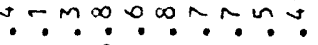
coon $0, y$.

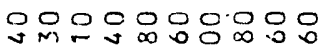
$\because \therefore \dot{\therefore} \therefore \dot{\sim} \div \dot{0}$

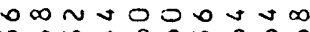
$\sin 20000000$ 0000000000 $\infty \sim \sim \backsim \sim \sim \sim \alpha \infty n$ $\because \therefore \because \therefore \dot{-1}$

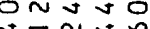

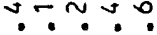

$--\sim \sim \sim \sim m \sim \sim \infty$ $\therefore \therefore \dot{0} \dot{0} \dot{\infty} \dot{\infty} \therefore \dot{0}$ $=-00 \infty \infty ⿻ 上$

$m \sim 0000 \% 000$ $\ddot{\sim} \dot{\infty} \dot{\infty} \dot{0} \dot{\sim} \dot{\infty} \dot{0}$ 두으.

0,0 ? $\dot{m} \dot{\sim} \dot{\infty} \dot{\sim}$ NNT-

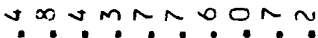

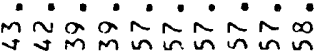

onoomuam?

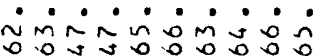

0000 $\dot{0} \dot{0} \dot{0}$

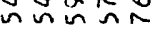

$-m n \infty \infty v \infty-\infty-$ nn $n \rightarrow \sim \alpha m i n \approx$ $\because \backsim y m-m-M \simeq \approx$

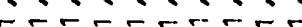

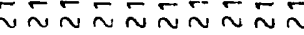
$a-n-a$

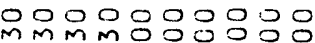
$\sim \sim \sim \sim n n \in n \in n$ $\sim \sim \sim \sim \sim \sim-\cdots$

$\infty \infty \infty \infty \infty \infty \infty \infty \infty$

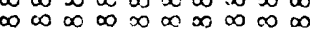

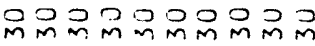
$\sim \sim \sim \sim \sim \sim \sim \sim \sim \sim$

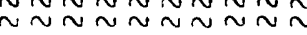

$\sim \sim \sim \sim \sim \sim \sim \sim \sim N$ $m \sim m n M m m$ In $M$

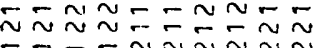

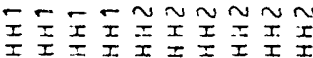

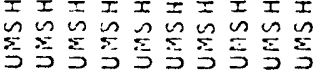

0 - n mond o d n nan

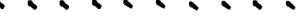

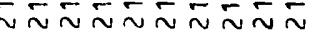

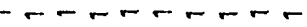

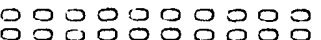
n n 00000000 - 00008900

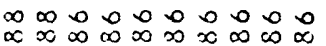

On $\infty$ or amavm $\because \because y m$ $\ddot{\sim} \check{\sim} \check{\sim}$ $r+r$

00000 웅ㅇㅇㅇㅇㅇㅇ $\infty \infty \infty \infty \infty$

글금음음을 $\sim \sim \sim \sim \sim \sim \sim \sim \sim \sim$

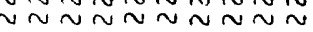

$\sim N N N \sim N N N N N$

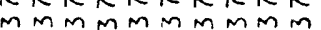

응음 nnแ n $\hat{m} \hat{M} \tilde{M} \tilde{M}$

$\approx \approx \Xi \Xi \approx \simeq \Sigma \tilde{\sim} \approx$ $\approx \approx-5=-\div ニ-5$

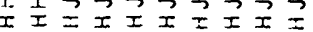

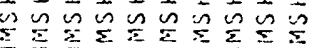

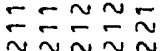
$\neg \neg \neg \sim \simeq$ $I I I I I$ $\sum \sum \sum_{0}^{n} \sum^{n} n$ -anutanga 


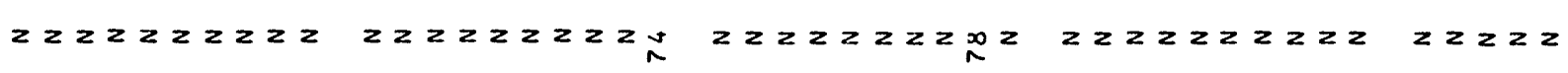

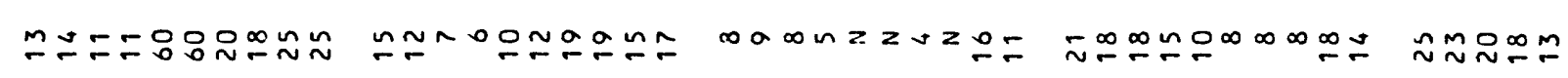

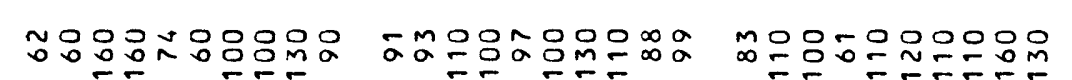

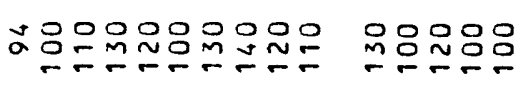

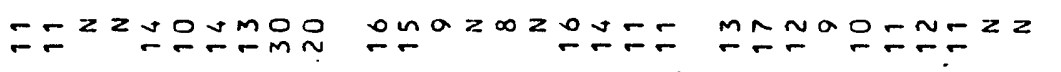

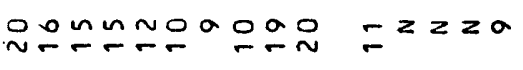

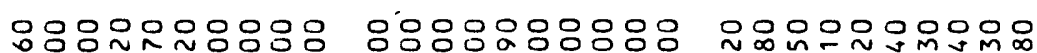

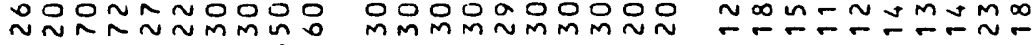

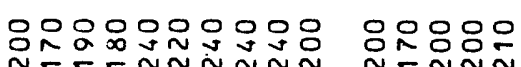
$\therefore$

$\div$

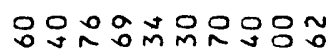

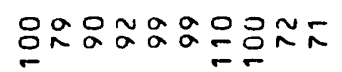

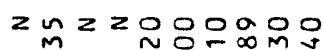

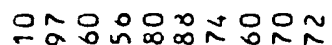

은요는

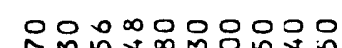

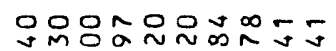

mm $m \sim n n m \sim$

$\because M O a \cong$

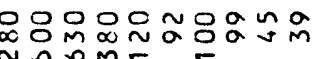

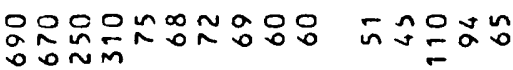

$\stackrel{\sim}{\sim} \tilde{\infty} \dot{m} \tilde{\sim} \tilde{m}=0 \tilde{N}$

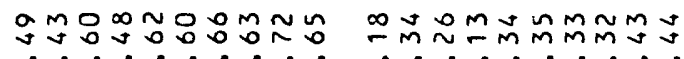

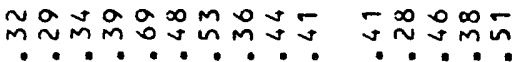

은ㄷㅇㅇ응응영응

miñ்

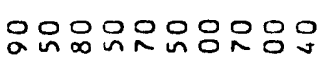

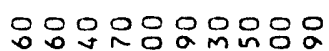

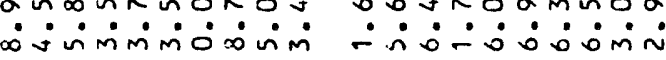

둥ㅇㅇㅇㅇㅇㅇㅇㅇ

$\because ?$. ?

음응요

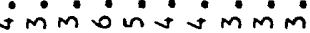

iminim

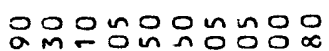

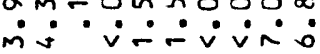

Oonsonnun on

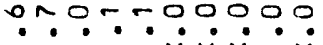

$00000 \infty \infty \pi n n$ กำ $\dot{0} \dot{0} \dot{0}$

nn 00 munn num o0 vvi $\dot{0} v \dot{v} v \dot{v} v$

nn nn nun i.

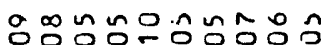

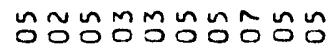

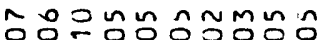

Nmasun

ำํำํํํํำำ

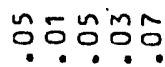

oodnonknan

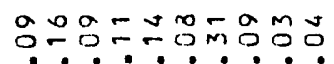

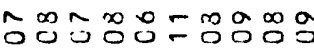

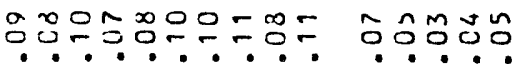

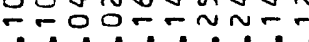

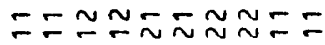

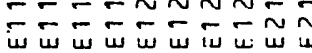

$\sim \simeq-\tilde{\sim} \approx=-\approx \sim$

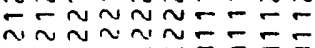

$\bar{\sim} \approx \approx \sigma=\simeq \simeq \bar{\sim}$ ํㅜㅆㅆ

T⿱一⿻上丨)

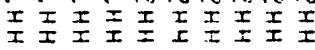

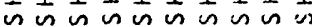

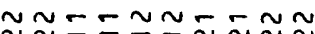

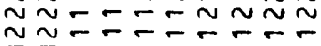

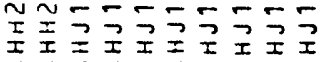

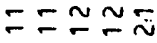

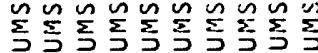

$\sum_{J}^{n} \sum_{j}^{n} \sum_{j}^{n} \sum_{j}^{n} \sum_{J}^{n} \sum_{J}^{n} \sum_{J}^{n} \sum_{J}^{n} \sum_{j}^{n} \sum_{J}^{n}$

$\sum_{J}^{n} \sum_{J}^{n} \sum_{j}^{n} \sum_{J}^{n} \sum_{j}^{n} \sum_{J}^{n} \sum_{J}^{n} \sum_{j}^{n} \sum_{j}^{n} \sum_{J}^{n}$

IIIII

$\sum_{\supset}^{n} \sum_{j}^{n} \sum_{j}^{n} \sum_{j}^{n}$ 


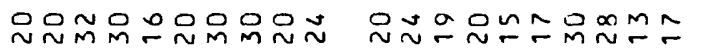

ㅇm웅ㅇㅇㅇㅇㅇㅇㅇ $=a \tilde{\sim} \sim \simeq \square \simeq \simeq \pm$

0000000000 $\sim \sim m m \infty$ -MMUn- $m$ M

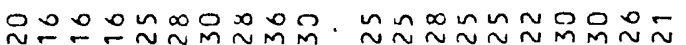

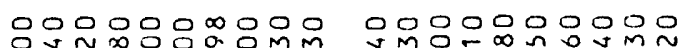

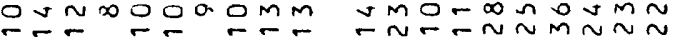

onoㅇo요 naำ

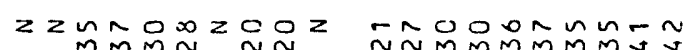

$\stackrel{\sim}{\sim} \tilde{\sim} \hat{\sim} z z z z \dot{m}$

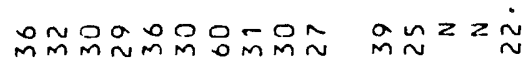

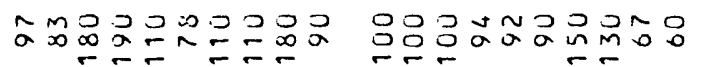

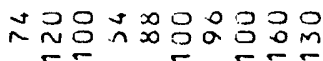

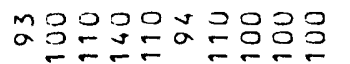

롱므응

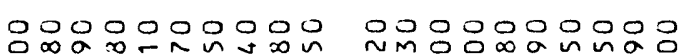

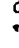

$a \sin ^{2}$

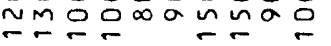

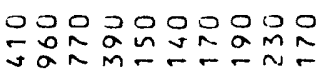

0000000000 ninacosano

00000

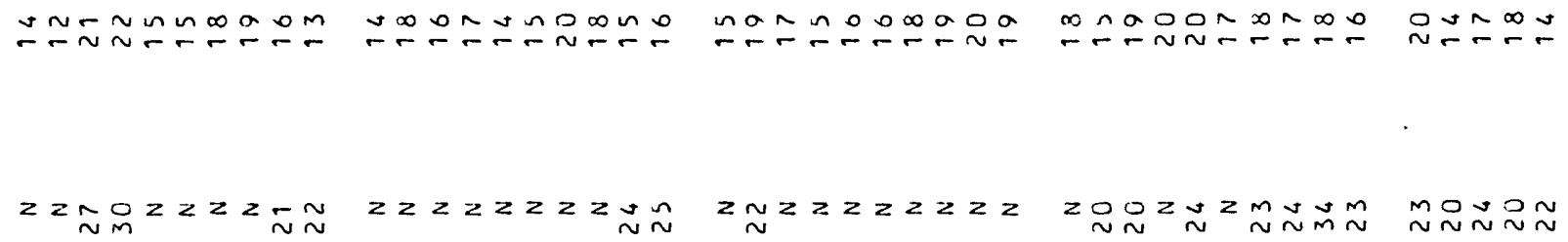

$z w z z z z 2 z z$

$200 Z \sim \sim \sim \sim N \sim N$

M유뮤

DOOn nODMOUm

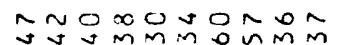

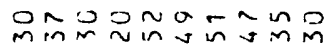

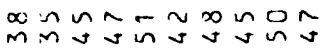

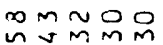

$\sim \sim-\sigma \sim \sim-\sim \sim$

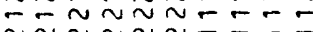

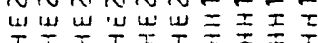

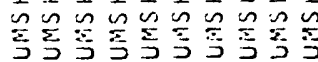

$\bar{\sim} \approx \approx \sim \Xi \approx \sim \bar{\sim} \bar{\sim}$ $\because \sim \approx \sim \bar{\sim} \check{\sim} \sim \tilde{\sim}$

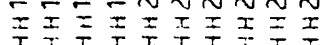

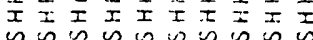

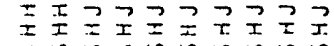

$\approx \approx=-\sim \sim \bar{\approx} \approx \sim$ $\approx \approx-\div \div \div \approx \approx \approx \approx$

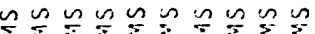

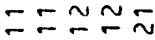
$\bar{\sim} \bar{\sim} \bar{\sim} \sim ⿻ 上$ $\vec{I} \vec{I} \vec{I} I$

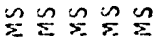

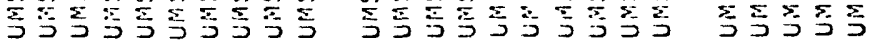


응으은응은운은으 $\therefore \therefore$ : $\dot{0}$ imm

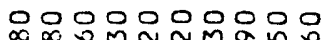
mijmminmi

กตm $0 \sim a 000$ mंñmiging

กMN- NOMONO जिimisisis:

क. $.9 m-M \sim \backsim 5$ -- $\dot{a} \dot{0} \dot{0} \dot{m}$

$000005 \pi M^{\circ}$ -miñminmín

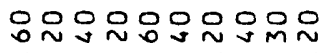

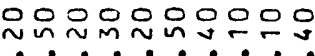

O0000000No กับ

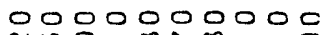
mi:

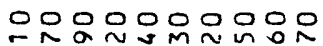
$\therefore$ - $\dot{\sim} \dot{\sim} \dot{-} \dot{-}$

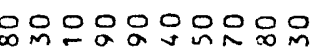

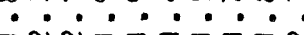

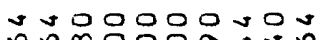
$\because 0,0.00 .5 .50$

manctoon

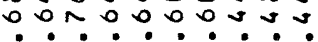

$00 \div \infty \sim 0 \div \because n$

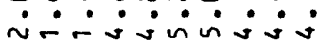

$\simeq \sim \sim \sim \infty \sim \ln a \infty$

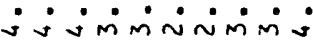

o 0 m nn a oOm

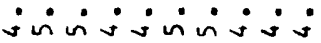

응ㅇㅇㅇㅇㅇㅇㅇㅇㅇㅇ은

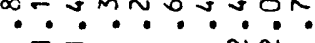
$\simeq \sim$

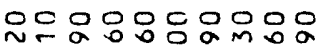

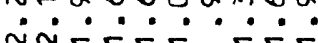

DOONDOONDN ก...

엉ㅇㅇㅇㅇㅇㅇㅇㅇ응

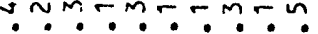

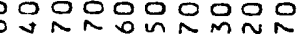

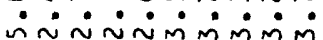

잉ㅇㅇㅇㅇㅇㅇ -

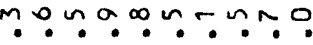

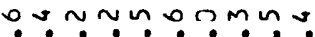

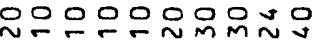

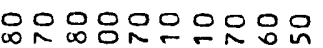
……ㄴ.

능ㅇㅇㅇㅇㅇㅇ ․․…

os onojomas on on on .ง.

tevornhtinn

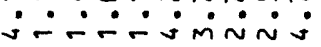

$\sin 0$ on isidid

monnmogona

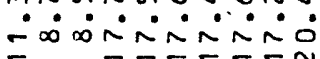

nunumentumon jo.jizinin

-moninonatad

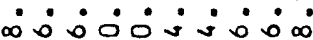

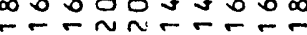

$\operatorname{coc} 00000 \pi, a$ $\because \dot{0} \dot{0} \dot{0} \dot{0}$ कำ

$m \sim n-m$ $\dot{a} \dot{0} \dot{n}$ neranaman

mMMกmo⿰N N

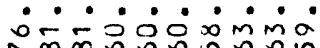

NOmv-ONNOM $\ddot{n} \dot{\sim} \dot{0} \dot{0} \dot{0} \dot{0} \dot{n}$

- a onmuaguo $\dot{\infty} \dot{\infty} \ddot{\hat{n}} \dot{\sim} \dot{0} \dot{m} \dot{m}$

monñun

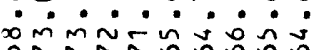

$0 \cos \infty$ $\dot{0} \dot{0} \dot{0}$

on. - Smm nmoo ปี่

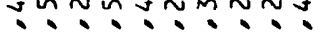

こぇ்

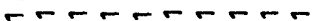

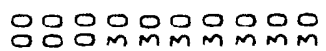
OOO $\sim N \sim \sim N \sim N$

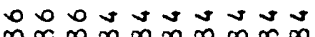

nodadonatana

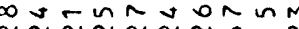
$\because \simeq \because \simeq \because \div \div \div \div$ $\bar{\sim} \bar{\sim} \bar{\sim} \bar{\sim} \bar{\sim} \bar{\sim}$ nann

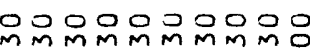

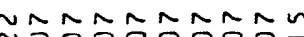

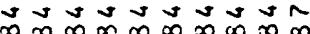

응응응응ㅇㅇㅇㅁㅇㅇㅇㅁㅇ un nnunun nun กヘกヘกヘกヘヘ MMMMMMMMMM

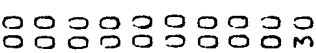

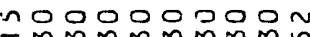

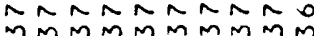

$\bar{\sim} \approx \Sigma=\approx \sim \Sigma \bar{\sim} \approx$ $\approx \approx N=\mp= \pm N \approx$ 可可可可可可至 $\sum_{j}^{n} \sum_{j}^{n} \sum_{j}^{n} \sum_{j}^{n} \sum_{j}^{n} \sum_{j}^{n} \sum_{j}^{n} \sum_{j}^{n} \sum_{j}^{n} \sum_{j}^{n}$

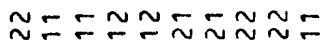

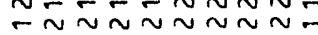
$\vec{I} \vec{I} \vec{I} \vec{I} \vec{I} \vec{I} \vec{I} \vec{I}$

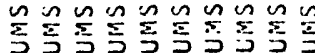

nตñ SOOMMMOCá

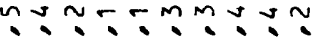
$\bar{\sim} \bar{\sim} \bar{\sim} \bar{\sim} \bar{\sim} \bar{n}$

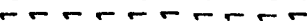

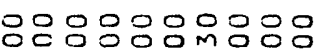
nun nn $n$ n 000 $\tilde{N} \approx \tilde{\infty} \tilde{\infty} \tilde{\infty} \tilde{\infty} \tilde{\infty}$

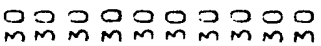
$\sim \sim \sim \sim \sim \sim \sim \sim \sim \sim$ แก ก ก ก

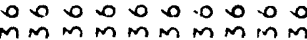

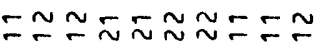

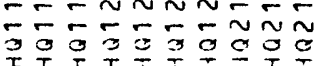

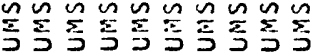

Dooganmnom n-OaOñon

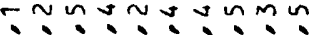
$\bar{\sim} \bar{\sim} \bar{\sim} \bar{\sim} \bar{\sim} \bar{\sim} \bar{\sim}$ añ

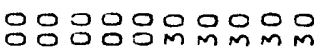
OOOOONNÑN

$\sim \sim \sim \sim \sim \sim 000$

MOOOOOOPO을 $\sim \sim \sim \sim \sim \sim \sim \sim \sim \sim$

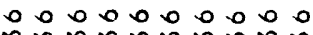

nกำ n이유 กำ? -

읐옹ㅇㅁ음 NNNOOO 00000 $\infty \infty \infty \infty$

M음을 $\approx \sim \sim \sim \sim \sim$ ํํํํํํำ

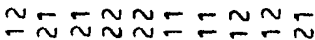

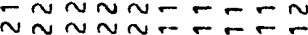

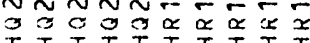

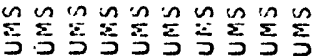

$\bar{\sim} \sim \tilde{\sim}=\sigma$ $-\sigma \pi \approx$

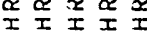
$\sum_{j}^{n} \sum_{j}^{n} \sum_{j}^{n} \sum_{j}^{n}$ $\infty \infty \infty \infty \infty \infty \infty \infty \infty$ $\bar{\sim} \bar{\sim} \bar{n}$ 


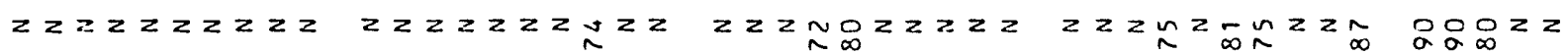

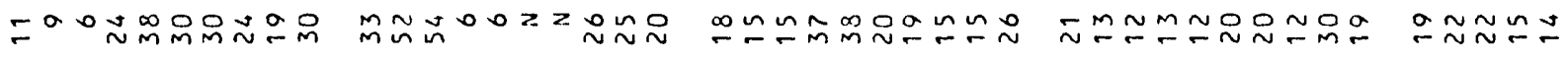

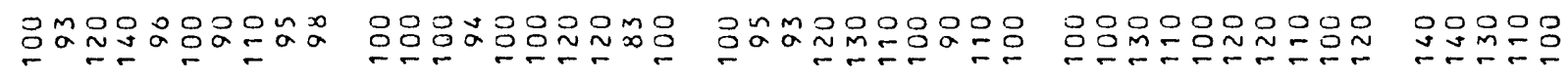

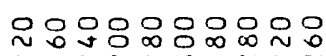

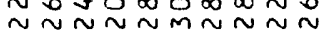

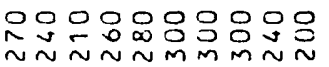

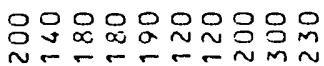

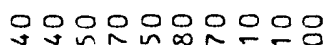
nับำ

monñ
mono

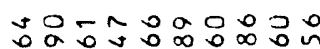
유요

응ㅇำ

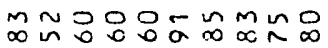

m

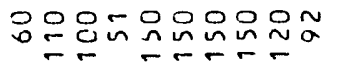

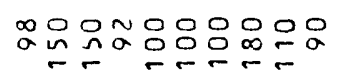

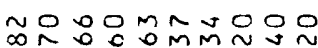

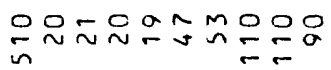

mヘッํํㅇํㅇ

ง 은

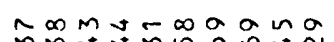

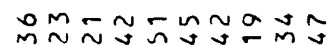

aำ뭉ำ

oñ $\operatorname{con} 0$

mm. vmmis.?

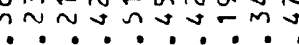

am. m.

……

in 00000000000

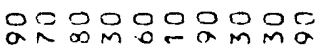

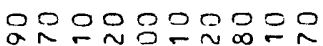

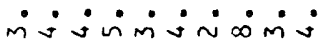

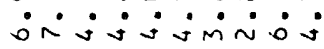

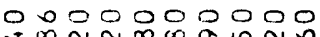

‘. $\therefore \sim$ inimi

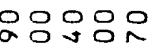

íง

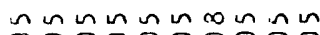

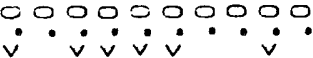

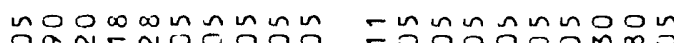

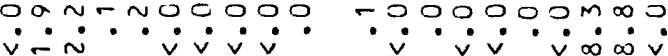

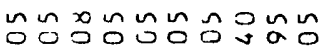

$\dot{v} \cdot \dot{v} v \dot{v} \dot{v} \cdot$

กำนnก

$\dot{i} \dot{v} \dot{v}$

ํํํํํำำำกำ

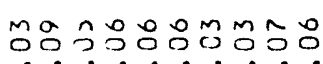

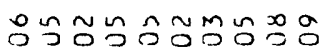

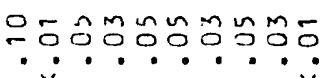

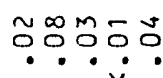

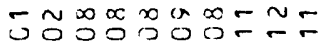

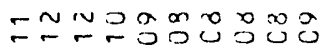

ำ

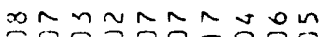

คูกดั $\checkmark$

$\bar{\sim} \approx=\mp \approx \sim \bar{\sim} \tilde{N}$

$\approx \approx \sim-\cdots-\cdots-\cdots$

$\vec{I} \vec{I} \vec{I} \vec{\equiv} \vec{I} \vec{I} \vec{I} \vec{I} \vec{I} \vec{\tau}$

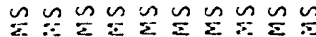

$\approx \Sigma=\simeq \approx \bar{\sim} \approx \approx=$ $\simeq \check{n} \approx \approx \sim \sim \sim \approx \sim$

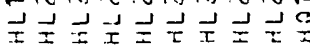

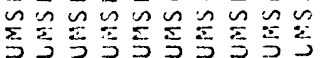

$\overline{2} \approx \bar{\sim} \approx \approx \approx \Xi \Xi \approx$ $\overline{3}=-\frac{\pi}{3} \overline{3} \approx \widetilde{2}$

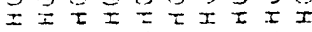

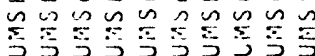

$\cong \bar{\approx} \approx \approx=\Xi \cong \approx$

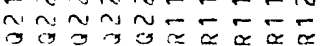
I $工=I I I I I$

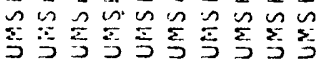

$\check{\sim} \sim \mp$ $--\sigma \sim \pi$

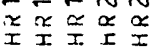

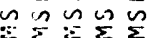




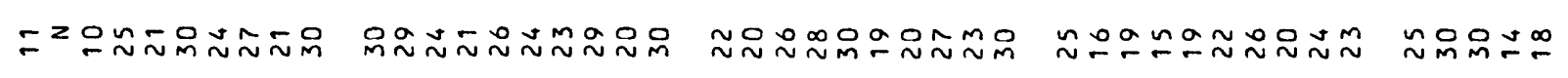

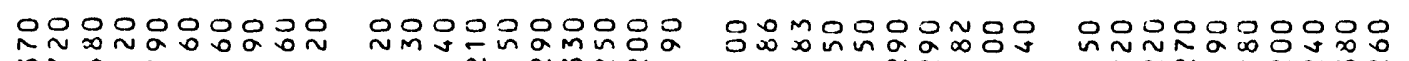

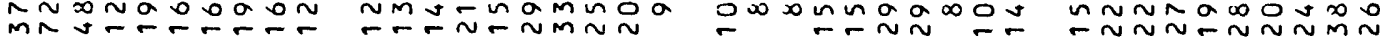

잉ㅇㅇㅇㅇㅇ

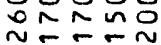

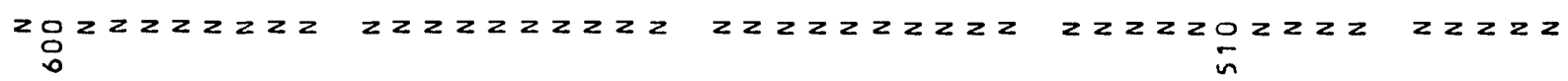

$z z z z$

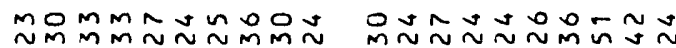

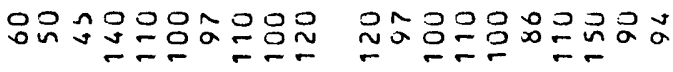

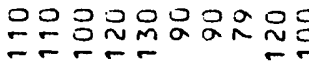

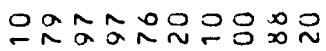

은유응음

\section{0} 음ำ

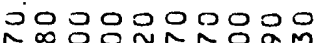

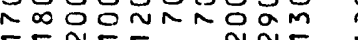

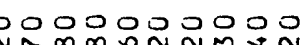

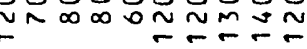

웅유음옷 $\because 2=m$

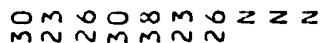

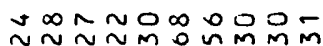

$\hat{\sim} \tilde{m} \dot{m} z \stackrel{n}{\sim}$

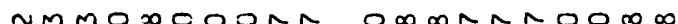
-

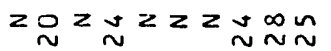

$\sim 22 z \stackrel{0}{\sim} 20 z \stackrel{\sim}{\sim}$

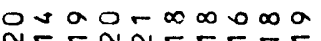

$\infty 00000 \ln \infty$

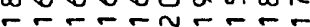

$a a \infty \sim \infty$

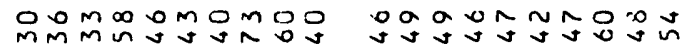

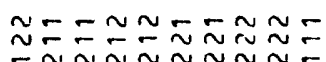

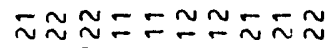
$\dddot{2} \sim 5 \pm=55$

in in in in in

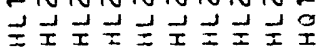

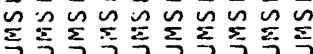

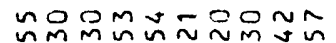

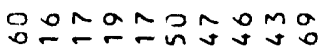

$=\simeq \simeq \bar{\pi} \approx \approx-\tau \sim$

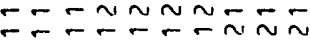

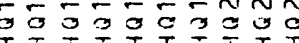

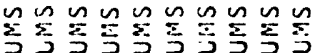

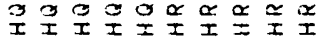

$\simeq \bar{\sim} \approx \approx \Xi \Sigma \simeq \simeq \bar{\sim}$ $\approx \approx \approx \approx \approx \bar{\alpha} \sigma \bar{\alpha} \bar{\alpha}$ 足品王呈呈

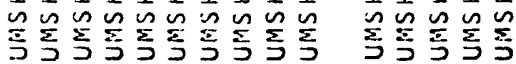

$\approx 0 \infty \sim \infty$

$\bar{\approx} \approx-\leftarrow$ $\approx \approx \sim \mp-$ 


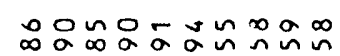
๑. $0.9 .9 n n$

0000000000

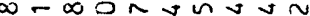
نที่งที⿻上丨

$0-\infty-\sim \sim m n-$

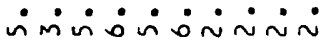

$m \sim 0 \infty \infty \sim \infty \sim 0$

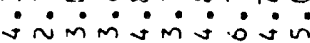

$m \infty a n m n 000$

பminmiñ $\dot{\sim}$

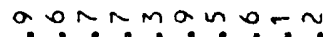

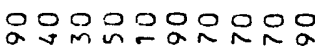
mंن्जiñن:

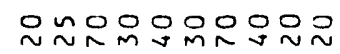

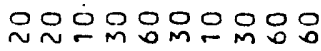

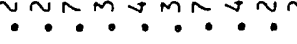

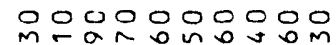
……… $\sim \sim \sim-$

0000000000 Y.? $0.0 \times-\because \cdots a$ - $\dot{m}^{\prime} \dot{v} \dot{\sim}$

옷ㅇㅇㅇㅇㅇㅇㅇㅇ으음유

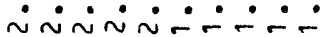

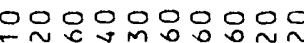

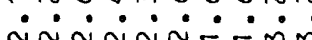

$\sin 0$

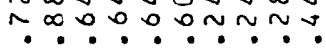

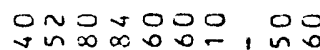

$\cdots \because \therefore \dot{\sim} \dot{\sim}$

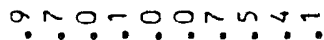

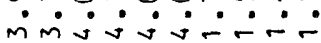

anopoodinno

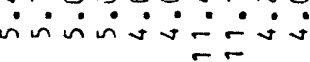

$\operatorname{natcosponos}$

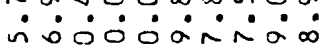
ำ

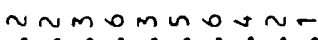

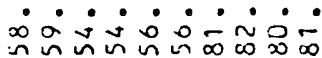

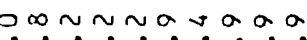

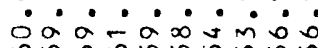

ononadom-ma $\dot{0} \dot{0} \dot{\sim} \dot{\sim} \dot{\infty}$

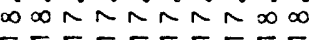

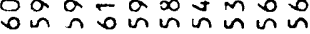

amommaanoa a $m \sim a m \infty 00 \sim m$ $\because \because \because m \div \div \div ! \div$ $\bar{\sim} \bar{\sim} \bar{\sim} \bar{\sim} \bar{\sim} \bar{\sim}$ - $\ldots-\cdots+\cdots$

응응응뭄믐응응

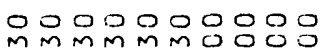

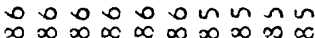

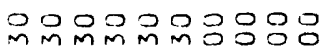
$\sim \sim \sim \sim \sim \sim \sim \sim n \sim$

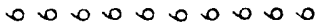
MMMMMMMMMM

$\sim \sim \Sigma \approx \approx \sim-\tau \sim \sim$

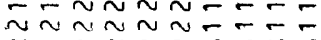

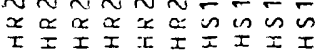

nandnom an

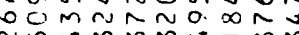
$\because \because \div M m \div \div M$ $\bar{\sim} \bar{\sim} \bar{\sim} \bar{\sim} \bar{\sim} \check{\sim}$ - $-\ldots-\ldots$

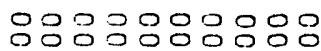

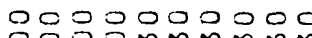

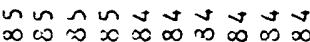

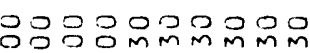
แนnกñกñ TU JUMMMMMM

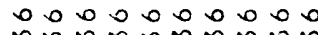

$\bar{\sim} \approx \approx \Xi \Xi \cong \simeq \bar{n}$ 프므들

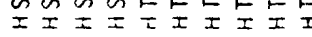

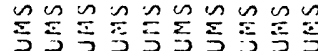

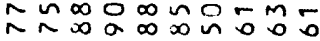
..・.・.!

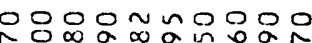

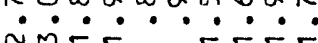

$\alpha a-n \infty \sim a 0 \infty m$ $\dot{m} \dot{m} \dot{\sim} \dot{\sim} \dot{\sim} \dot{\sim} \dot{\sim} \dot{\sim}$

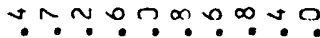
$\sim \sim \dot{\sim} \sim \dot{\sim} \dot{\sim} \sim$

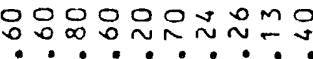

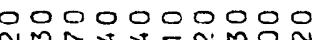
$\dot{m i} \cdot \because \infty \dot{0}=$

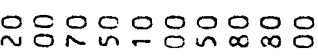

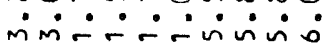

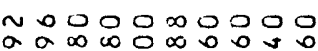
$90,00 \infty .00 .0$ $\because \therefore \because \because \therefore \dot{\sim}$

ปn on 0 mon in $N$

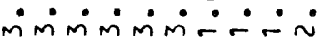

a ona ono 0 in $\dot{m} \dot{0} \dot{0}=\dot{0} \div \dot{\circ}$

No0000nv n -

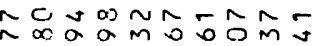
$\approx \stackrel{\alpha}{\sim} a m m M \sim O M$ $\because \because \because M m \because 、 ? m$ $\bar{\sim} \bar{\sim} \bar{\sim} \bar{\sim} \bar{\sim} \bar{\sim}$ $\simeq$

응ㅇㅇㅇㅇㅇㅇㅇㅇㅇㅇㅇㅇㅇ Oํำnnununn

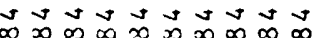

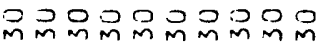
$\tilde{\sim} \sim \sim \sim \sim \sim \sim \sim \sim \sim$ o o o o O DOD O

$\approx \approx E=\simeq \approx \bar{\sim} \approx \approx \sim$

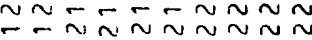

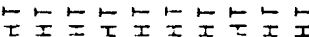

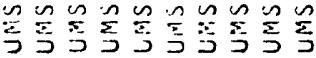




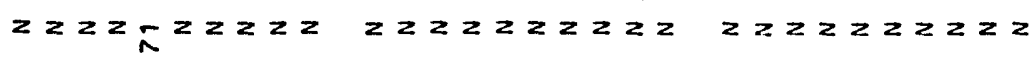

늠ำ

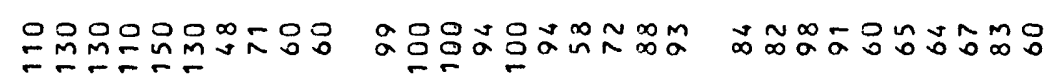

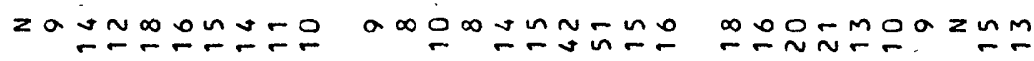

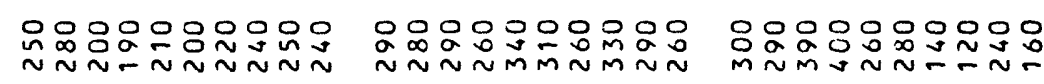

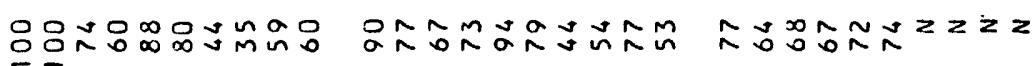

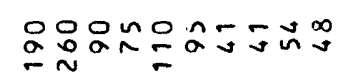

등응응응ㅇㅇㅇ요은 in:

은응음응ㅇㅇㅇㅇㅇㅇ응

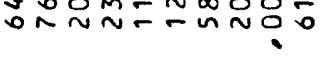

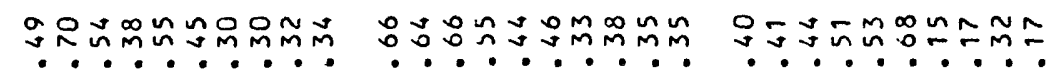

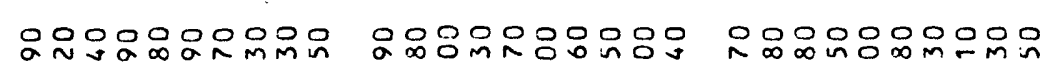

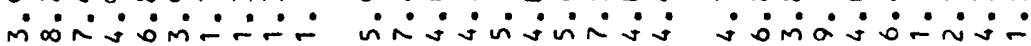

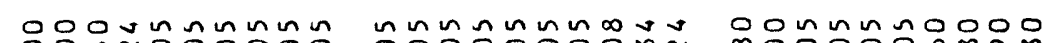

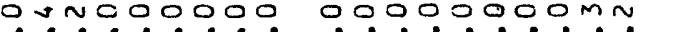

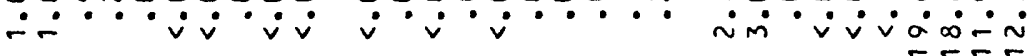

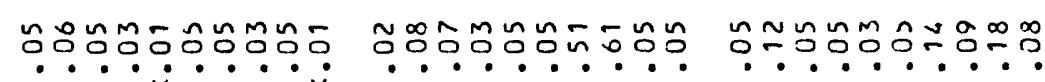

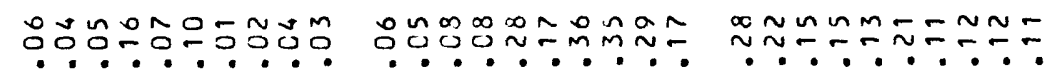

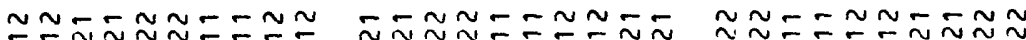

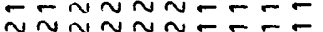

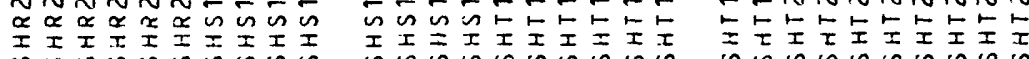

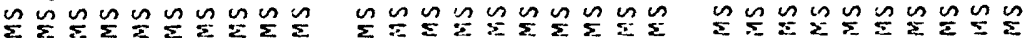




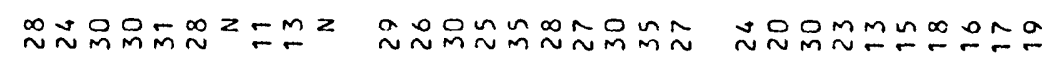

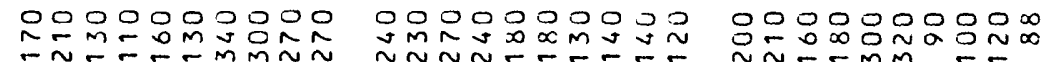

$z z z z z z z z z z \quad z z z z z z z 0_{0} z \quad z 0 z 2 z z 2 z z$

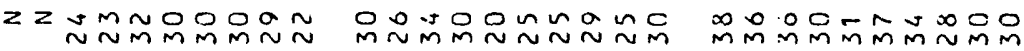

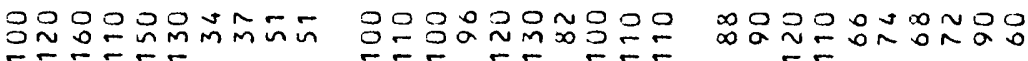

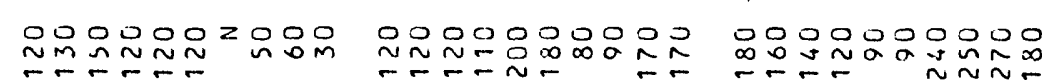

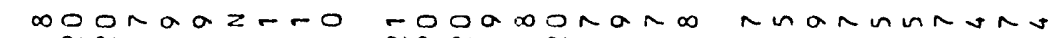
-nnman is

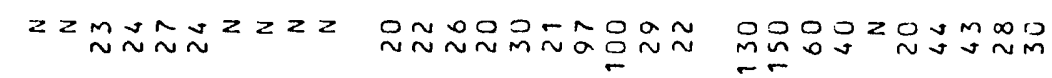

$\bar{\sim} \approx \approx \Sigma \Sigma \approx \approx \Sigma \bar{\sim}$

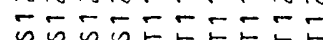
T工

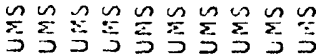

$\tilde{\sim}=\tilde{\sim} \sim \tilde{\sim} \tilde{\sim} \tilde{\sim}$ $\approx \approx \bar{\sim} \bar{\sim} \approx \sim \approx \approx \sim ⿻ 上 丨$

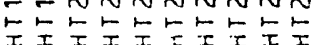

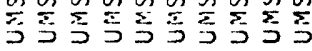


$\stackrel{\sim}{\sim} \sim-\infty-m-n \infty 0 m$ $000 \infty, a=\infty \infty \infty, \infty$ i

Pिo00n000

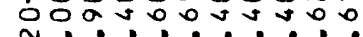
In $\sim \sim ⿻$

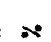

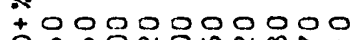
I.jomini

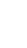

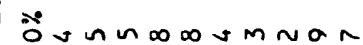
a

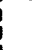

$\ddot{0} \sim \infty 0000000$ $\underset{z}{\sim} m m m \sim \because z \sim \pm \sim ?$ ( )

¿

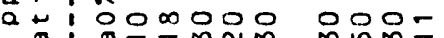
딘.?

ह E !

岁: :

는응응ㅇㅇㅇㅇㅇㅇㅇㅇㅇ ¿ ! !

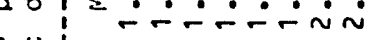

¿

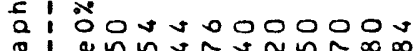

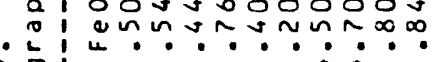

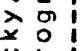

ว

-

c

$x$ n a cumiññ்$=0 !$ (4)

(1)

ज̆: ì

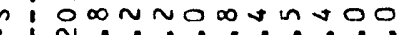

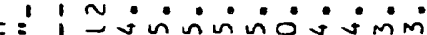

c $<\sim n \sim N-\ldots N-5$

$\therefore \therefore$ i

$>\pi 1 \times$

c

uै

c e nununumonun

a is

-

- 1 z

:

¿

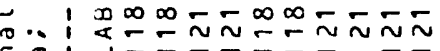

的

$+\infty$

-

'

¿ :

미 :

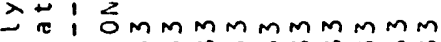

.

?

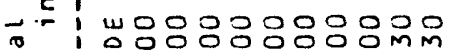

Sn $000000000 \mathrm{~mm}$

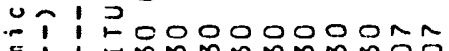

E! I

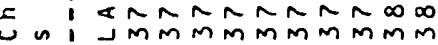

i:

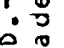

\&: $\approx \simeq \quad \approx \approx E=$

- $\frac{1}{2}=-\frac{\bar{w}}{w} \tilde{w} \sim \frac{N}{w} \tilde{w}$

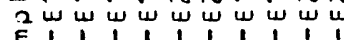

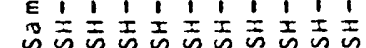
a a a a a a a a
0000040000 บับ $\div-000 \div$ $\sim \sim ⿻ \div \div \div \div$

0000000000

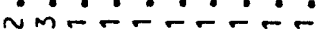

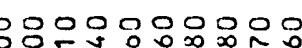
ón่ -

a amm-nn $n-n$ - imísimiñ

0000000000

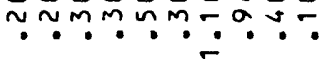

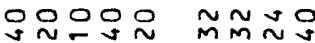
… 은은응웅응응언우

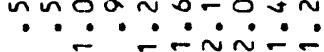

ONDONOODON 0 on $n$ in 0 ?

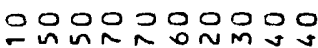

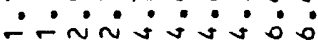
- intisisogo

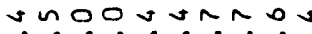

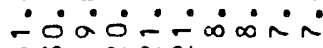
mMーn

moson mm-n

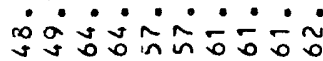

- o onnamann muำ $\because \because \div \div \div \div: \div \div$ $\bar{\sim} \bar{\sim} \bar{\sim} \bar{\sim} \div \infty \bar{\sim}$ -

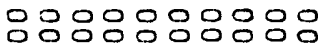
nununnunun r n $m m m m m m \sim \sim \sim \sim$ $\infty \infty \infty \infty m \infty \infty \infty \infty$

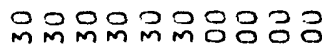
กิกิกี่์nnn $\infty \infty \infty \infty \infty \infty \infty \infty$ $M M M M M M M M M$

$\simeq \approx \bar{\sim} \tilde{\sim} \tilde{\sim} \simeq \simeq$

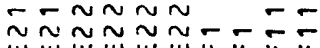
世

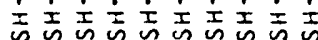
a a a a a a a a

$\infty$ no 0 o $\infty \infty 000$

? $\div-\div \dot{0}$

- $-0000 \sim \sim \sim 0$ a a 0000 in 000 . !. ! ! ! a. ..?

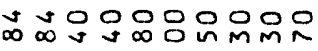
$\because \because \dot{-} \dot{\sim} \dot{m} \dot{m}$

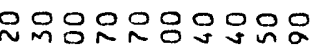
nivisisin

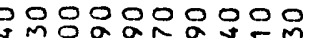
ㄴ.

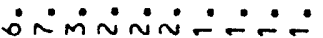

o용요

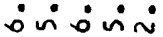

$\dot{m} \dot{m}-\infty \dot{m} \dot{m} \dot{\sim} \dot{\sim} \dot{\sim}$

nno0000000

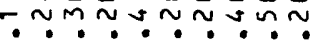

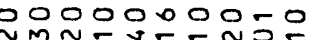

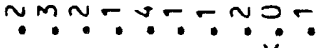

butimanctao

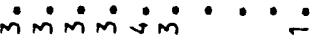

namn $\dot{m} \dot{v} \dot{m} \dot{\sim}$

00000 $\because \div \div$

는응ㅇㅇㅇㅇㅇㅇㅇㅇㅇㅇ응 ท‥ 도.

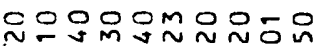
……

은유온

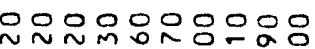

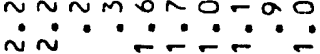

$00000-0000$ O $\infty 0 \infty$ o n $N M$ $\therefore \therefore \cdot \cdots \cdot$.

응융ㅇㅇ $\because \div \div \div$

oogannnman $\because \because \div \div \infty a m m \infty n$

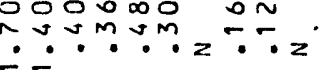
$0>000$

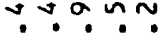
응ㅇㅇㅇㅇㅇㅇㅇㅇ응 onnuasa

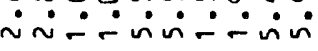

0000000000 a 0 mun om мंmiñ $\dot{\sim} \div \because \div$

00000 $0 \% \div \div$

ก ก க் $\dot{0} \dot{0} \dot{0} \dot{0} \dot{0} \div$

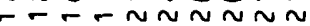

ommamuan-

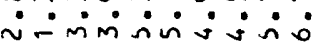
$\sim \sim-$ ก

$-v \infty \sim m$ ல் $0 \dot{0}$ $\sim N-n-$

aง.0.0.?.0.0.

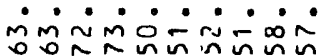

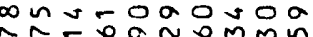
กิธง $\because \sqrt{1} \cdot 1$.

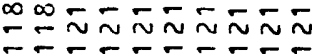

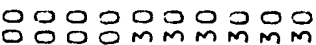
nun $n \sim \sim \sim \sim \sim \sim$ $\checkmark v \checkmark m m m m M m$

$\sim \sim \sim \sim \sim \sim \sim \sim \sim \sim$

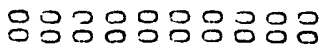
nnnnmunnnn $\infty \infty \infty \infty \infty \infty \infty \infty \infty$ $m M m m m M m m m m$

$\approx \approx \Sigma=\approx \sim \bar{\sim} \bar{\sim}$

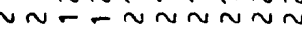
$\div \div \div 4 \div \div \div \frac{1}{1}$

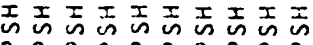
a a a a a a a a a $a$
ก $\sim 0000 m \infty n$ $\dot{0} \dot{0} \sim \dot{\sigma}-\dot{\infty} \dot{0} \dot{0} \dot{0}$

on $00 \sim$

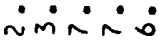

oamanun a o o

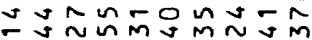

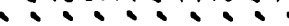

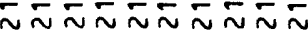
-

$0-n \sim \infty$ $\approx \tilde{m} \simeq \simeq$ $\because \because \div \div$ $\bar{\sim} \bar{\sim} \bar{\sim} \bar{n}$ MOOOOOOOOOOOOOOOO $\sim \sim \sim \sim \sim \sim \sim \sim n \sim$ MmO0000000 $\sim \sim \infty \infty \infty \infty \infty \infty \infty$

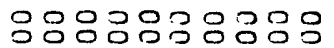
nunum nnum nu $\infty \infty \sim \sim \sim \sim \sim \sim \sim \sim$ $M M M M M M M M$

$\approx \approx \Xi \Xi \simeq \sim \bar{\sim} \approx \approx \sim$ $\sim \approx \bar{I} \bar{I} \bar{I} \bar{I} \bar{I} \bar{I}$

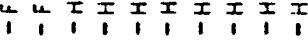

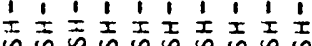
a. a a a a a a a

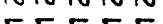

MOOOOO $\sim \sim \sim \sim \sim$ $\sim \sim \sim \sim \sim$

글음은 $\sim \sim \sim \sim \sim$ $\sim \sim \sim \sim \sim$ $M m M m m$

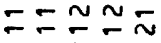

$\because \simeq \sim \sim \sim$ 111 贡贡贡贡

a a a a $\infty \infty \infty \infty \infty$ 


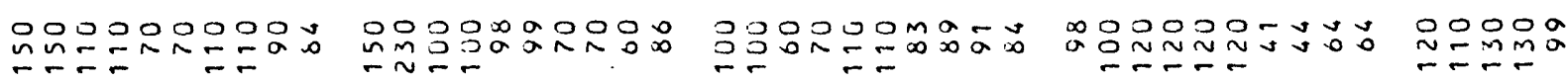

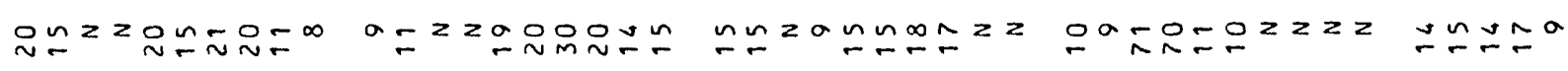

$z z z z z z z z z z z z z z z z z z \quad z z z z z z z z z \quad z z z z z z z z z$ zzzz

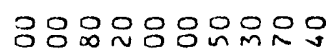

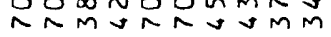

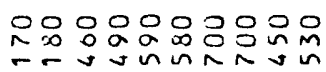

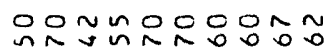

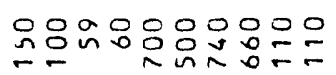

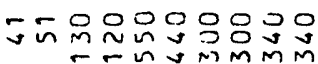

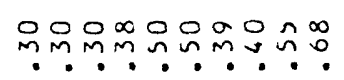

80

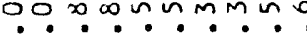
$\therefore$

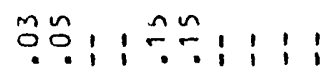

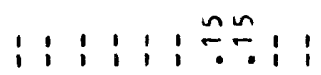

ำ1:ヘำ: $1:$

$1: 1: 1: \because 91:$

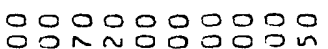
ñminiviom

0000000000 $\because \cong \because$ m.

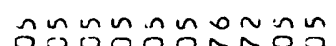

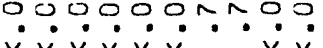

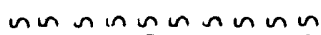

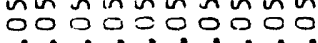
$\dot{v} \dot{v} \dot{v} \dot{v} \dot{v} v \dot{v} v$ ธำด์

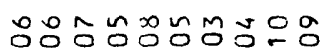

$m m \approx \infty \approx a m \sim m n$ $\simeq \simeq \Sigma \bar{\approx} \approx \quad \simeq \simeq$ $\bar{\sim} \tilde{\sim} \approx \tilde{\sim} \approx--\bar{z}$

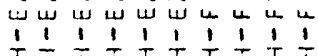

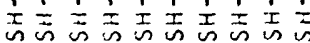

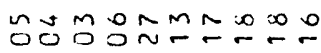

옹응음앰ㅇㅇㅇㅇㅠ

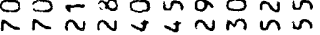

운음옹옹음용 vmmmm-..-

응ㅇㅇ웅으 ตำ的的

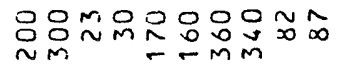

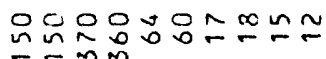

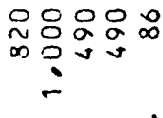

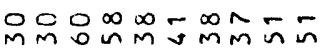

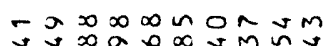
m.ำ.ำ.ำ.

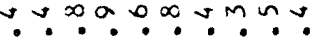

$\backsim \infty \circ \sim a$ ง $ง \backsim ?$

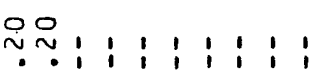

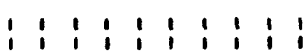
$: i: i:$ $\because 01: 1: 1: 1: 1:$

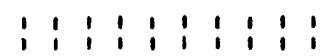
: $1: 1: 1:$

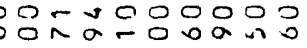

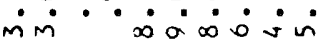

0000000000 ogoon in inmog

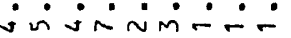

00000 modas कंजिए.

กนกนกั

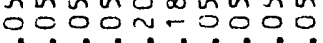
$\dot{v} \dot{v} \dot{v} \cdot \cdots \dot{v} \cdot \dot{v}$

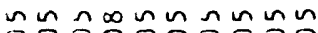
oodogoo $\dot{v} \dot{v} \cdot \dot{v} \dot{v} \dot{v} \dot{v} v$

$\hat{ก} น \infty \sim\{$ $\dot{v} \dot{v} \cdot \dot{v}$

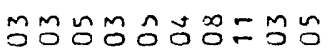

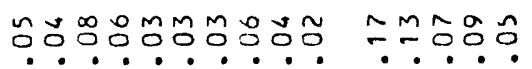

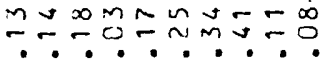

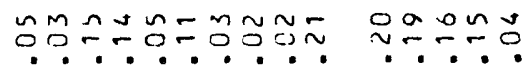
$\approx \approx \Sigma=\approx \sim \Sigma \bar{N}$

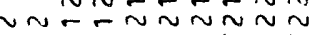

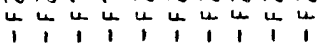

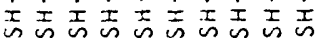
$2 a \mathfrak{a} a$ a $a$ a a a

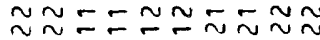
$\approx \sim ⿻ 上 丨 \bar{x}$

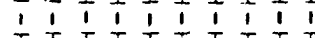

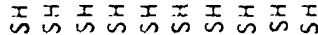
a a a a a a a a a
$\Sigma=\simeq \simeq \Sigma$ $\tilde{I} \tilde{T} \tilde{T} \tilde{I}$ 1111

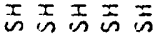
a $a$ a $a$ 


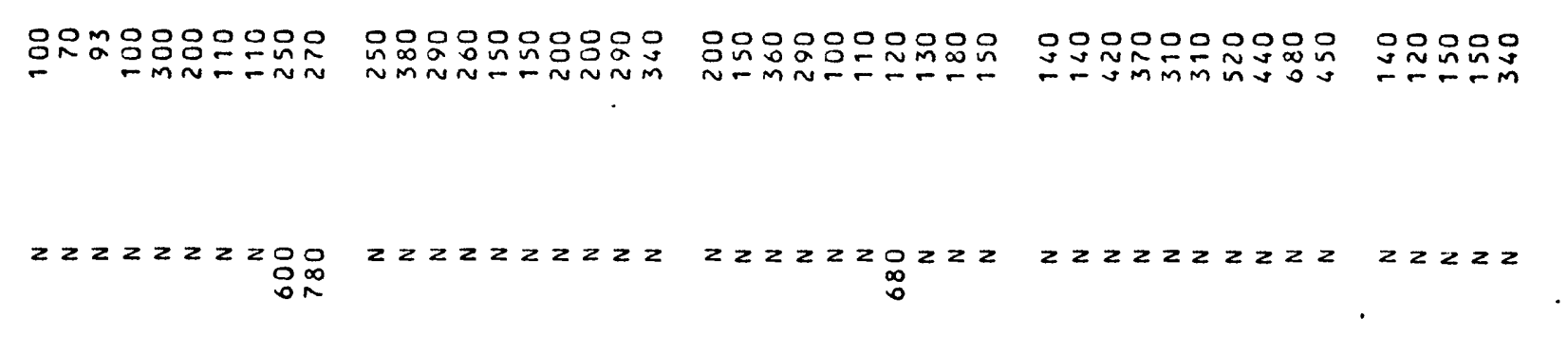

열

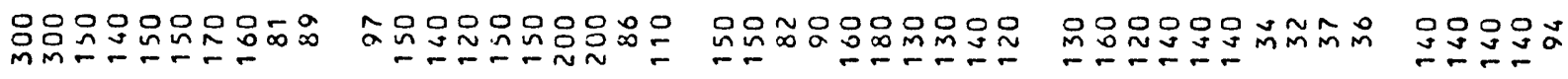

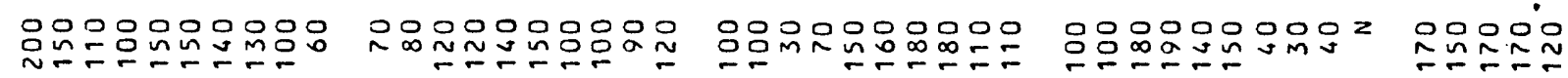

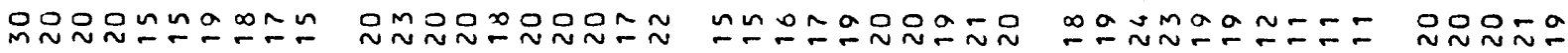

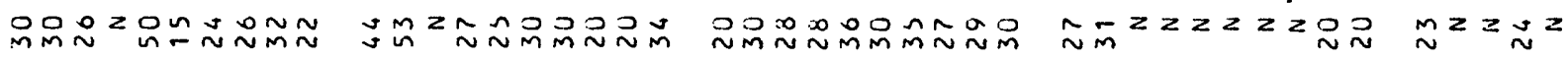

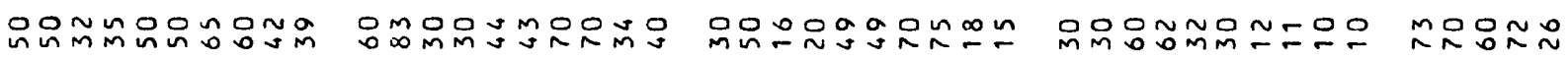
:

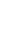

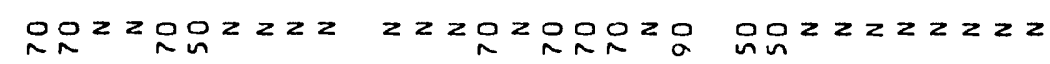

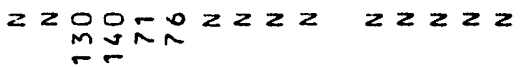

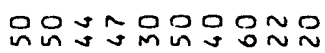

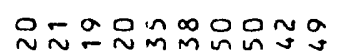

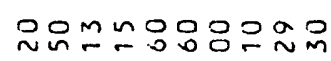

$\stackrel{\infty}{\sim} \underset{m}{\infty} \sim 002 z \ln z$

오ำำ

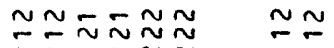

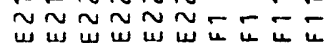

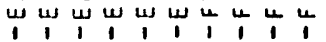

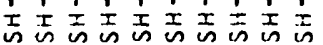
a a a a a a a a $\approx \approx=-\approx \sim \bar{\sim}$ $\sim \sim-\pi N \sim \sim \sim \sim N \sim$

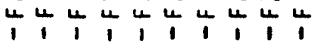

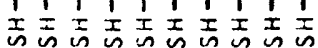
a a a a a a a a a a $\approx \tilde{N}=\Xi \tilde{\sim} \tilde{\sim} \tilde{\sim}$

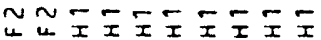

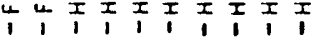

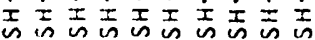
a a a a a a a a

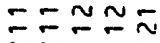
$\tilde{I} \tilde{I} \tilde{I} \tilde{I}$ $\begin{array}{llll}1 & 1 & 1 & 1\end{array}$

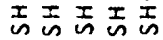

a a a a 


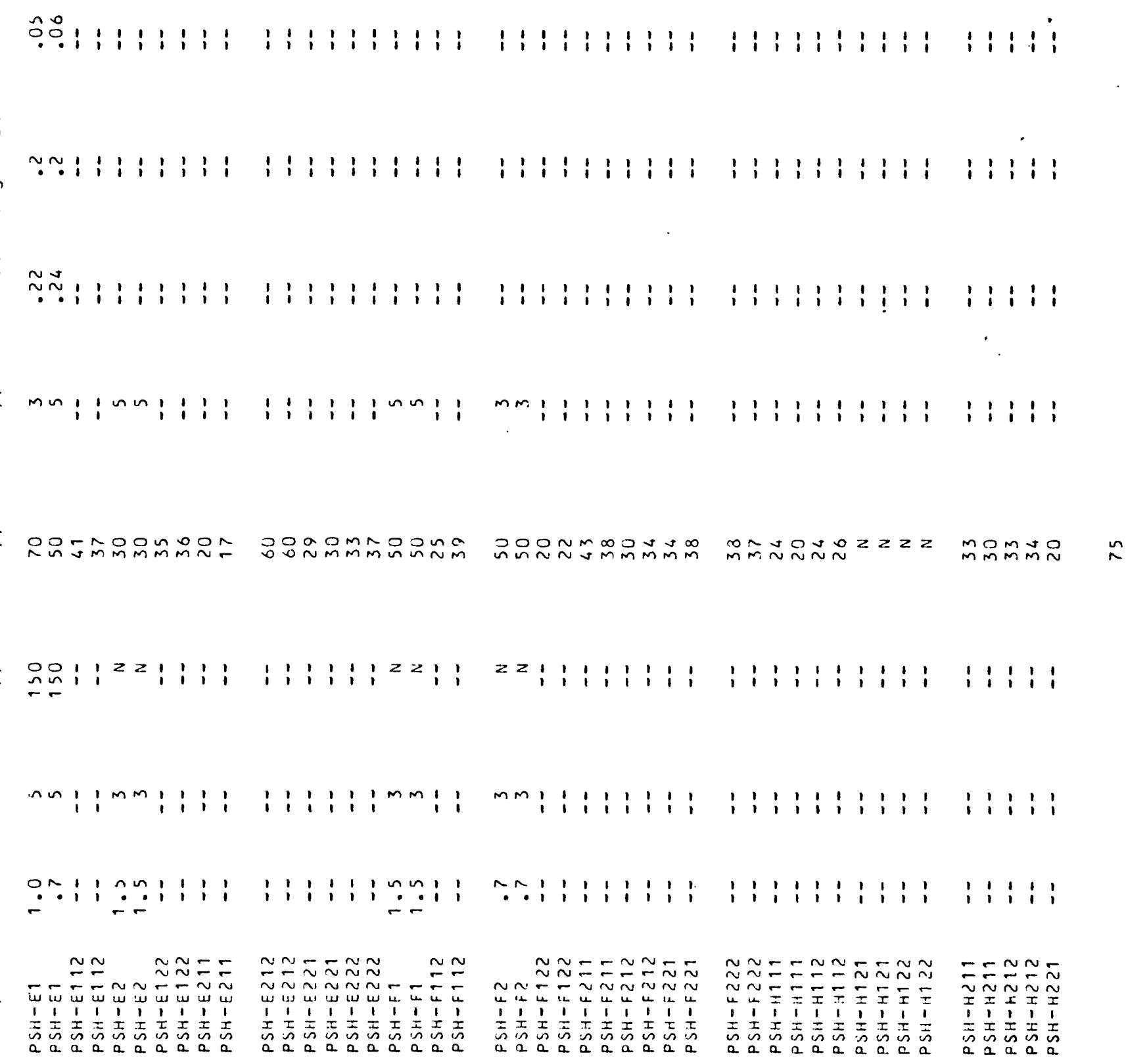




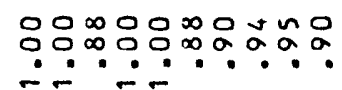

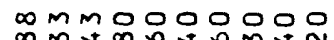
$\infty m v \infty 040 m, 4$ $\because \because \dot{\sim} \dot{-1}$

응ㅇㅇ응응ㅇㅇㅇㅇㅇ응 m- "rimisini

o-aroavmos míminimí.

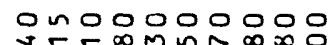

บ.?

onooornooo

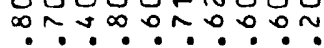

o on-mon- 00

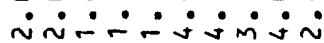

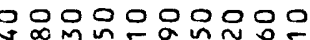
mंminm

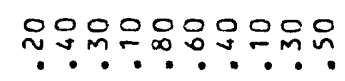

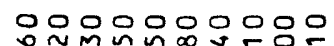
ㄴ.?ㄴ.?

N O0000NNN $m z \sim \backsim m n \infty m m m$

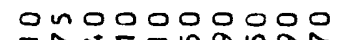

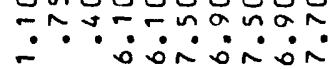

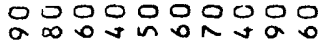

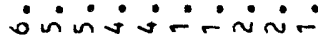

$\sim \infty 0-\sim m \infty \sim-0$

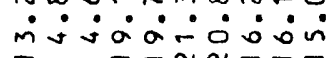
- $-\sim \sim T-$

ง vmoan-on

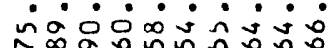

-mananonmn mMำกำ约正 m!?:?!m!m? $\bar{n} \bar{\sim} \bar{n} \bar{n} \bar{n} \bar{\sim} \bar{n}$ 므므.

있잉ㅇㅇㅇㅇㅁㅇㅇㅁㅇㅇㅇ음 N N N0000000

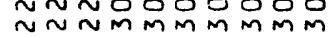
$\infty \infty \infty \hat{\infty} \hat{\infty} \hat{\infty} \hat{\infty} \hat{\infty} \hat{\infty} \hat{\infty}$

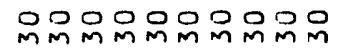
$\sim \sim \sim \sim \sim \sim \sim \sim \sim N$

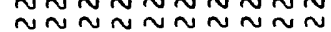
ñÑÑÑÑÑ $M M M M M M M M M M$

$\bar{\sim} \tilde{\sim}=E \sim \sim \Sigma \bar{\sim} \sim$ $\approx \tilde{N} \bar{\sim} \check{\sim} \tilde{\sim} \tilde{N}$ x工ェ

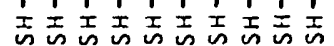
a a a $a$ a a $a$ a a

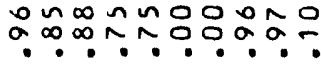

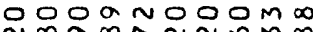

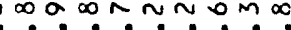
$\because \therefore \div \div \div$

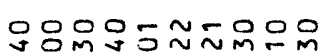
$\because \cdot \therefore \cdot$

응ㅇㅇㅇㅇㅇㅇㅇㅇㅇㅇㅇ응 $\because$ …………

O $\infty \sim \infty \sim \sim \infty 000$ $\because \sim m \sim m \cup m$ n $?$ ? $\dot{\sim}$.

ovonaso- on mi்ட்- $\dot{m} \dot{\sim} \dot{0} \dot{0}$ m-r.ñN

0 om $000 m m n$

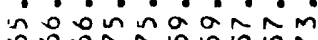

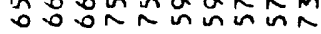

n unomavon

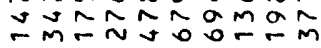
…………

$\approx \bar{\sim} \sim \bar{\sim} \sim \infty \sim \bar{\sim} \sim$

응ㅇㅇㅇㅇㅇㅇㅇㅇㅇㅇㅇㅇㅇㅇ On nn n n nnn nn n

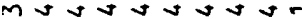

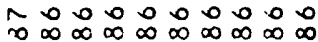

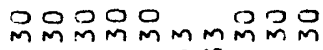
$\approx \sim \sim \sim \sim \infty \infty \sim \sim \sim$ $\approx \sim \sim n \sim n \infty$ in 0 in 0 $\sim \sim \sim \sim \sim \sim \sim \sim \sim N \sim$ MMMMMMMMMM

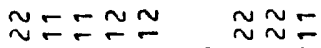

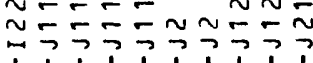

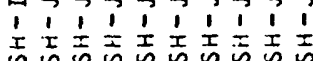

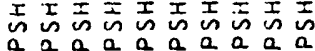

v $\quad 10 \infty \infty 000$ $\because z z \sim m \cup \sim m \cup v$

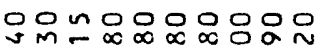
ง..

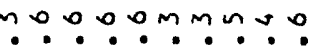

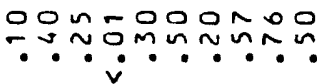

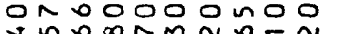
$\because \because \div \infty \sim m \sim \circ \div \sim$

$\sim-a N \sin -\infty \infty$ n

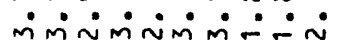

$\infty \infty \infty$ iñن்

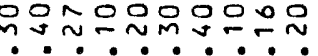

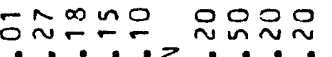
$\because \cdots \cdot 2 ! \cdots \cdot$ ํํㅇ 등ㅇㅇㅇㅇㅇㅇㅇㅇㅇㅇ $\infty$ ? $\because \because \ddot{\because} \ddot{\circ}$

00000 and

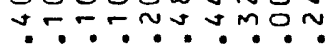
$\because n \frac{1}{\square}$ !

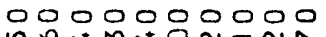
กับmง ำ $\because \because \dot{\sim}$ iñmimím

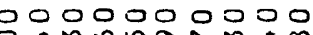

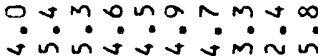

000000 is is:

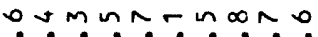
$\dot{\sim} \infty \dot{\sim} \dot{\sim} \dot{0}=\dot{0} \dot{0} \dot{0}$

$\sim \infty \checkmark v 0 \infty \sim a n$ inं $\dot{0} \dot{\infty} \dot{\infty} \dot{n} \dot{0} \dot{0}-\dot{-}$ r a - n n

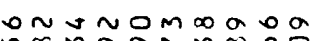
$\approx \infty m a \sigma N m \infty 00$ $\because \because m \div \div \div ! ? \div !$ $\bar{\sim} \bar{\sim} \bar{\sim} \bar{\sim} \bar{\sim} \infty$ -

음응음응영응

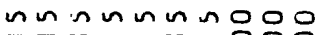
$00000 \infty 0 \checkmark ు$

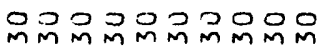

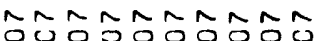

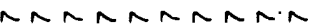
$M M M M M M M M M M$

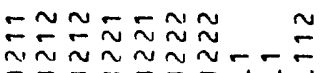

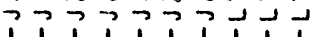

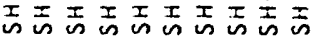
a a a a a a a a $\sin \pi-n \operatorname{sana}$ $\therefore \sim \dot{\sim} \dot{0} \min \dot{m}$

$\because \sim \sim m-\sim a n m m$ ति $\dot{0} \dot{0} \dot{0} \dot{i} \dot{0}$

morva iñن:

toonenumutan $\because \because \because \div \div ก ! บ$ $\bar{\sim} \infty \bar{n} \bar{\sim} \bar{\sim} \bar{\sim}$

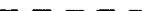

$-m m \sim \infty$ m:บ? $\bar{\sim} \bar{\sim}-\infty$ $n-2=0$

응옹임음응응응응응

응응응요 잉잉ㅇㅇㅇㅇㅇㅇㅇㅇㅇㅇㅇㅇㅇㅇㅇ

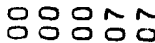

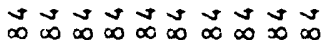

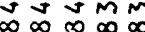

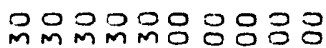
$\hat{0} \tilde{0} \tilde{0} \tilde{O} \operatorname{mnn} n$ OOOOOO-

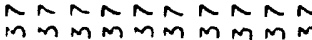

잉옹ㅇㅇㅇㅇㅇㅇ음 nn nn n $\hat{m} \hat{m} \tilde{m} \hat{m}$

$\simeq \quad \tilde{\sim} \tilde{\sigma}=\tilde{\sim} \tilde{\sim}$

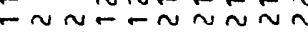
$\overrightarrow{\mid} \overrightarrow{\mid} \vec{\imath} \vec{\imath} \overrightarrow{\mid} \overrightarrow{\mid} \overrightarrow{\mid} \vec{t}$

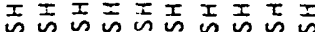
a a a a a a a a $\check{\sim} \tilde{\sim}$

$\tilde{U} \mathfrak{\mathcal { N }} \bar{\Sigma}$ त) $\overrightarrow{1} \sum_{1}$ 兵贡壳贡 a. a a a

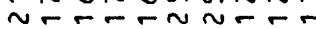
웅ำ ciñ. 


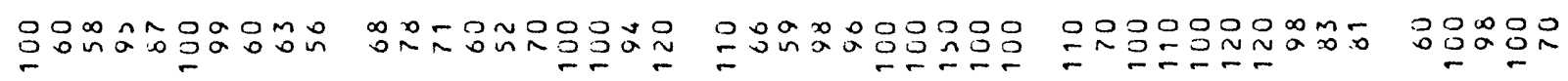

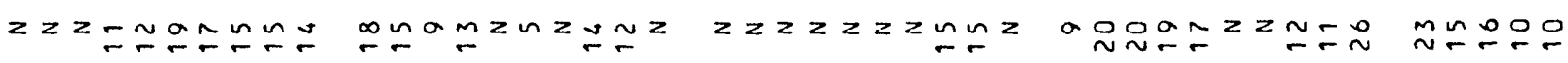

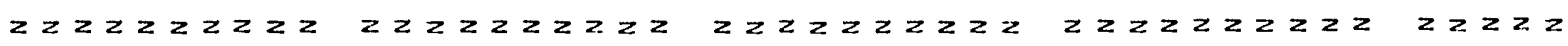

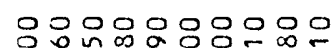

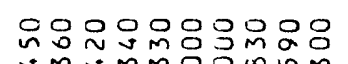

응웅ㅇㅇㅇㅇㅁㅇㅁㅇㅇㅇㅇㅇㅇ

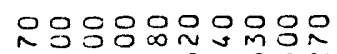

융요응옹

m-nog 0 $\therefore$

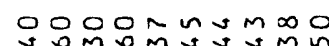
n-

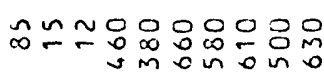

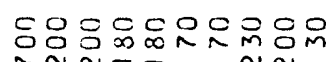

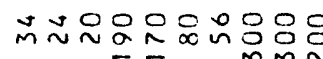

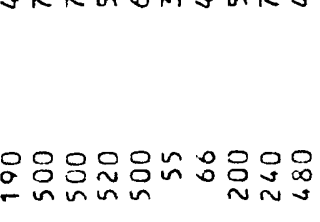

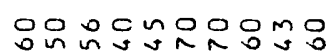

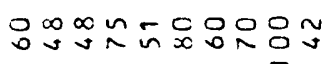

$\sim 00 \infty$ on $\sim 0 \operatorname{mn}$

잉요요의

$m-\infty m 0\} \sim \infty \sim a \quad \sim m \infty-\infty 000+\infty$

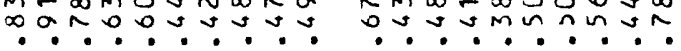

osingnmucoso

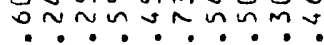

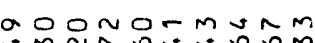

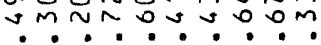

$\sim \sim 200$ mกษ

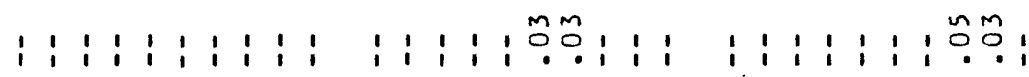

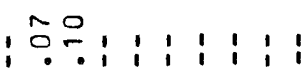

: $:$ กั

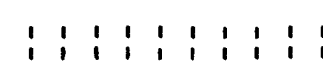

1: $1: 1, n m ! 1 !$

$1: 11111901$

!ก๊! :

$1: 1 ?$

의 $\min 0000000$ $m \backsim 00-m \in \infty$ - íríaí

0000000000

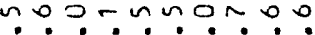

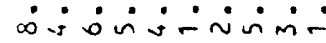

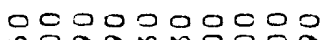
กO. $\because \because$ isminin

0000000000

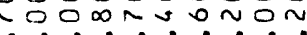
insonmmini

00000 - 000 i.

nununinununum nosunununumun 0.000000000 $\dot{v} \dot{v} \dot{v} \dot{v} \cdot \dot{v} v \dot{v}$

๓̊m

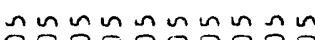

.000 .000 .0

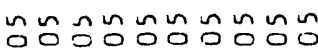
vivivivivivi

กำ nn n $\dot{v} \dot{v} \dot{0} \cdot$

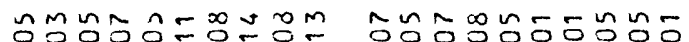

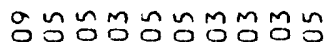

ิㅗㅇㅇํㅇํำกักำ

웡 .․․․․․…?

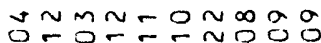
… $\cdot \dot{v} \cdot \dot{v}$

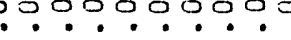

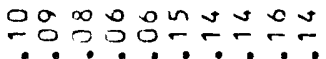

$\hat{A} M \simeq$ n $n \cap n=m$

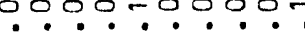

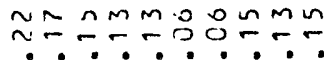
$\because \frac{\square}{\div} \div$

i!

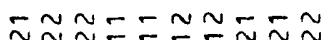

$\approx \approx \approx \bar{n} \approx \bar{\sim} \approx \approx \approx \pi$

$x \geq \pm \sim \sim \sim \sim \sim \sim$

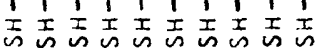

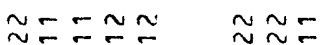
$\approx=\Xi \pm \approx \sim \sim \approx \sim \pi$ $\overrightarrow{1} \vec{i} \vec{i} \vec{i} \overrightarrow{1} \overrightarrow{1}$

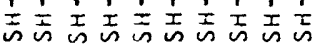
a a a

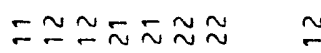

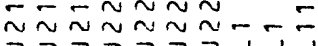
$\vec{\imath} \vec{\imath} \overrightarrow{1} \vec{\imath} \overrightarrow{1} \overrightarrow{1}$

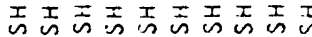
$a$ a d a casa a a $\approx \quad \approx \approx \sigma=\approx \sim \pi$ $\Xi \sim \sim \sim \Sigma \bar{\sim} \approx \bar{\sim}$

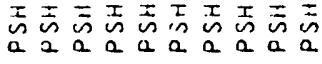
$\overrightarrow{1} \overrightarrow{1} \overrightarrow{1} \overrightarrow{1} \overrightarrow{1} \overrightarrow{1} \overrightarrow{1} \overrightarrow{1}$ $\bar{\sim} \tilde{\sim}$ $\sim N \sim=\Sigma^{2}$ $\overrightarrow{1} \overrightarrow{1}+\sum_{1}$

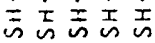
a ล ล ละ a 


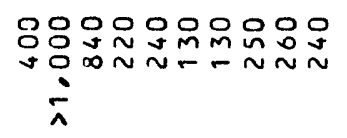

- 0 vonm n N N जU

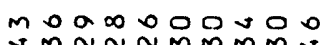
M N N NMMMUs

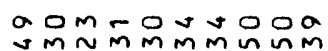

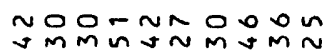

은은은

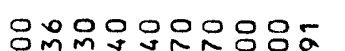

응오숭ㅇㅁ은은

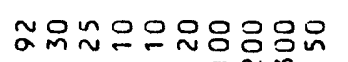

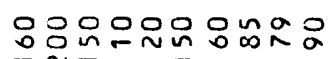

인옹응 M N

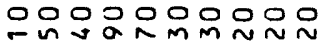

욱응응잉염원응

응의응영응응영

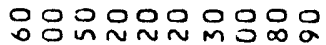

읏응으문 (1)

manOOOAn

은ํํ융ำ

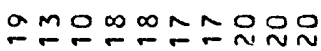

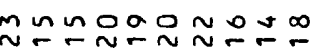

웅요온

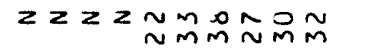

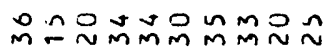

z픔ㅇㅇㅇ

\section{nNOOO-N nNm}

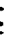

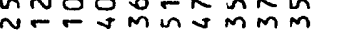

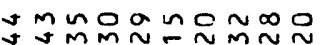

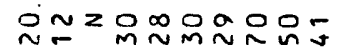

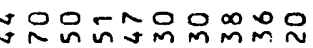

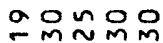

$z \geq z z 2 z 2 z z$

$z z z z z \operatorname{lol}_{\text {ก }} z 20$

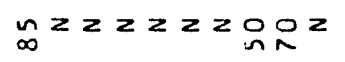

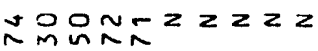

$z<2$ 응요

mozo요요

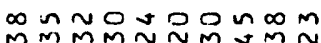

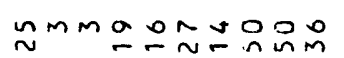

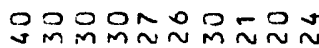

쑹요

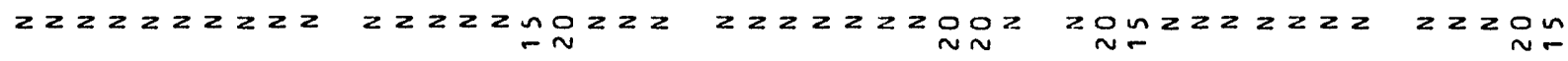

MMMMN NMUMN

(1)

$\bar{\approx} \approx \Sigma= \pm \simeq \Sigma \approx \approx$

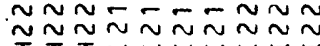

Ixエ

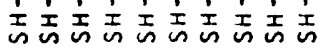

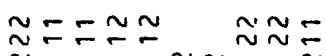

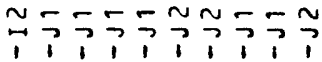

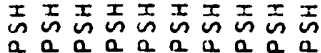

$\Sigma \simeq \simeq \bar{\sim} \approx \approx$

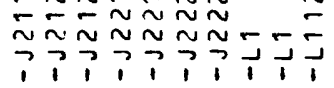

吉吉吉贡吉吉志志志

a a a a a a a a cáacaca a a $\bar{\approx} \approx$

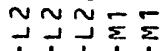

吉志志志志 


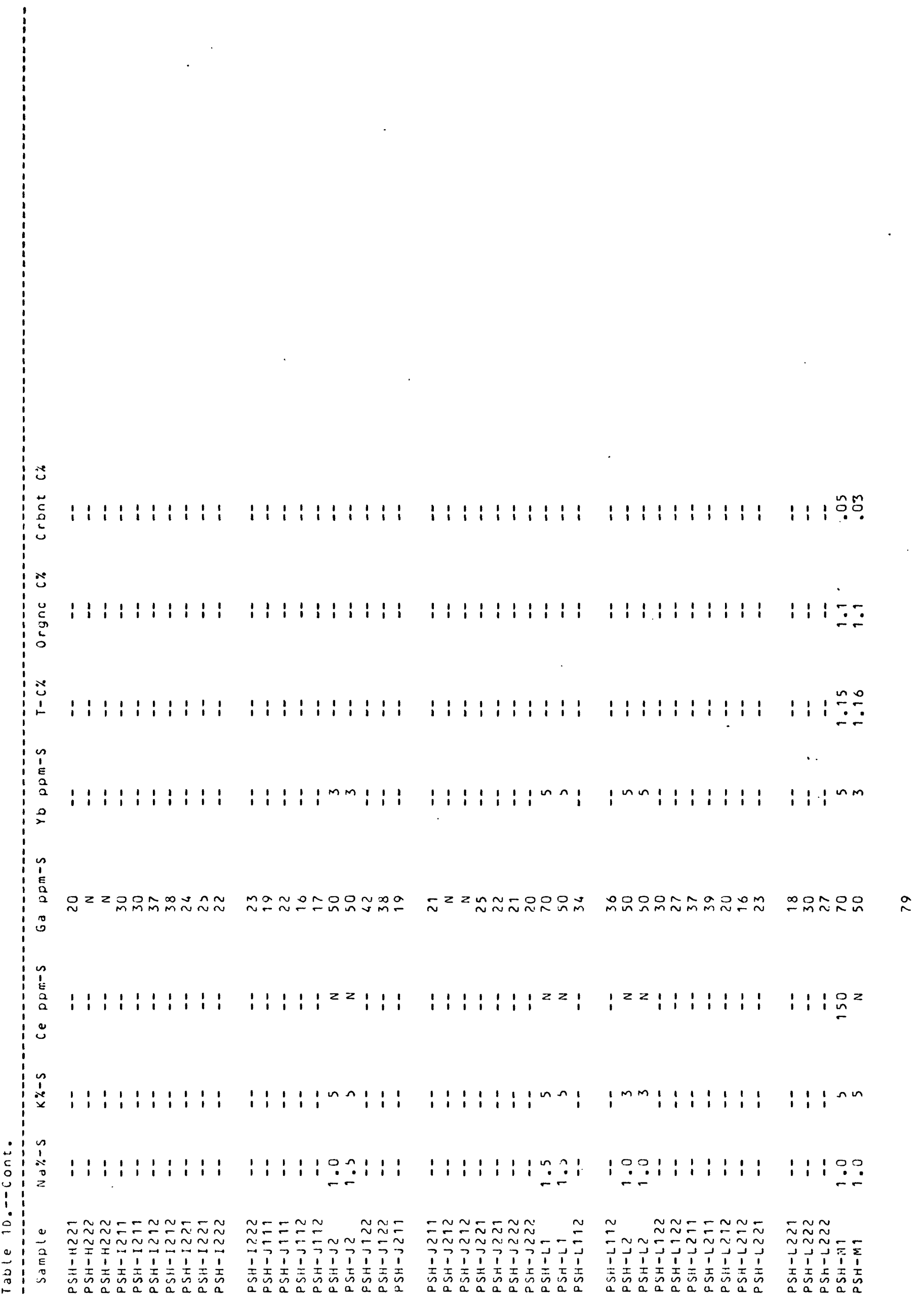


$\stackrel{2}{\circ}$

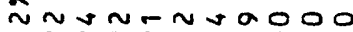
응. 00000000

Е・・・・・・ㅁ

in

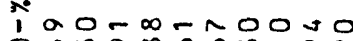
กำ. ……ำ.

I

is

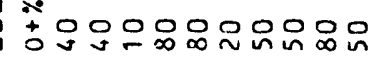

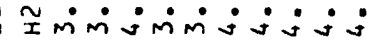

oै $\infty-m \backsim \sim 0 \sim \infty m m$ $\tilde{x} \dot{\sim} \dot{\sim} \dot{\sim} \dot{\therefore} \dot{\sim} \dot{m} \dot{m}$ i

öco0000000m

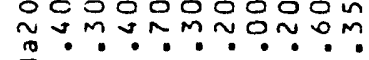
$\pi^{2}$

i.

ஜ̊o 0 N $0-0000$

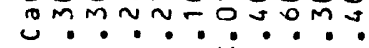

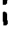

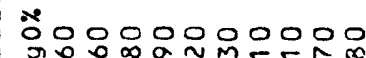

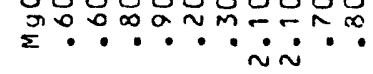

ì

茴的ง

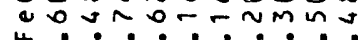
แ.... $\cdot \dot{\sim} \cdot$ ino000000000 o-m $n-$ - 0 on

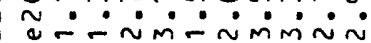
i 4

in

ôanam nun on ínijimiojá 1 i

$\therefore$

$\ddot{\sim} v \infty \sim m-\sim m \sim \sim a$

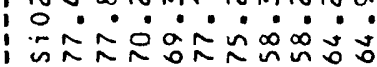
i

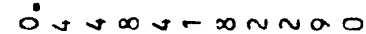

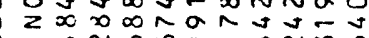

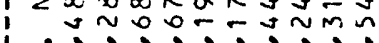
$\mathbb{a} \bar{\sim} \bar{n} \infty \bar{n} \bar{\sim} \bar{\sim} \bar{n}$ บN. i

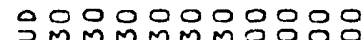

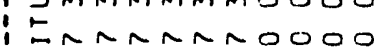

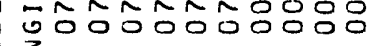
Znmmmmmmmmm $\downarrow \infty \infty \infty \infty \infty \infty \infty \infty \infty$

닝응ㅇㅁㅇㅇㅇㅇㅇㅇㅇㅇㅇㅇ

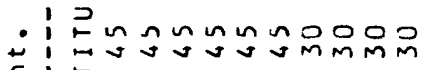

c: - JMMMMMmMMmM i i

$\therefore \approx \approx \approx \sim \approx \sim \sim N$

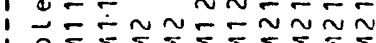
4 $2 \sum_{1} \sum_{1} \sum_{1} \sum_{1} \sum_{1} \sum_{1} \sum_{1} \sum_{1}$ D $\vdash$, $a$ a $a$ a $a$ a $a$ a $a$

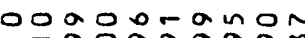
$\because 0000000 \infty$

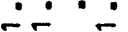

oon 0000000 m Na-m n v n m

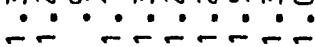

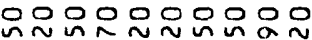
jisंminisin

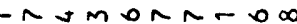

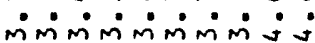

0000000000 $\because \div \div \div \div ? . ? \infty$

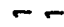

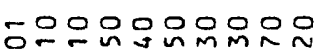

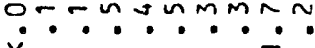

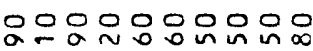
$\because \because \because \because \therefore \div$

$\checkmark \sim \infty \infty 000000$

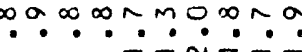

0000000000 moganoon-o

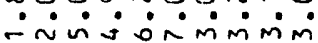

- nov unno: $\dot{\infty} \dot{\infty} \dot{0} \dot{0} \dot{0} \dot{0} \dot{0}=$ $\sim \cdots \sim N-\cdots \sim$

$-a n \pi v a n a t o$ $\dot{0} \dot{0} \dot{0} \dot{0} \dot{0} \dot{0}$

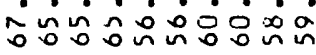

$\infty \infty \sim m \infty \sim \infty a \sim$ $m \sim-\infty \sim \sim \sim \sim \alpha \bar{N}$

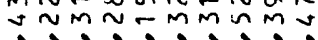

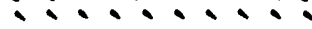
$\bar{\sim} \bar{\sim} \bar{\sim} \bar{\sim} \bar{\sim} \bar{\sim} \bar{\sim}$ $r-r+r+r)$

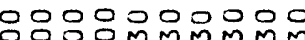
0000NNNNNN D0000000ิ00 $m m m m \sim \sim \sim \sim \sim \sim$ $\infty \infty_{\infty} \infty \infty \infty \infty \infty \infty$

영음뭄욤음음 Do00000000 MMMMMMMM MM $\sim \sim \sim \sim \sim \sim \sim \sim \sim N$ $M M M M M M M M M M$

$\bar{\sim} \approx \approx=\mp \approx \sim \bar{\sim}$ $\cong \cong \cong \approx z \bar{z}=\bar{z}$ 1111111111

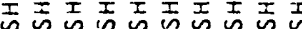
a a a a a a a a

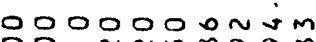

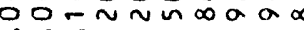

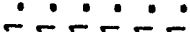

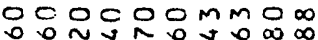

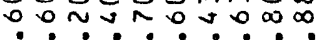
$\because \div \div \div \div \dot{0}$

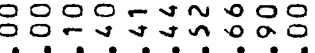
$\therefore \dot{m} \dot{-} \cdot \dot{0}$

은응 $\ddot{-1}-\dot{0}$

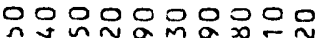
jंن் $\dot{\sim} \dot{\sim} \dot{m}$

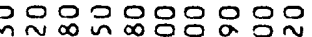

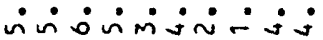

웅응응옹요 mi் $\dot{0} \dot{m} \dot{m}$

a $0 \sim a \checkmark+\infty \cup \infty \sim$ mi $\dot{0} \dot{m} \dot{\sim} \sim \dot{\sim}$

n n 00000000 $\because$ n $0 \infty m \sim 0, ?$ n in m r m?

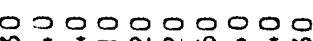

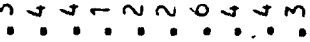

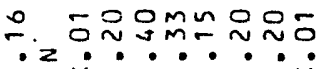
온으음워 . $\cdot$.

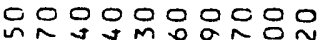

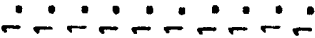

$000000000 \sim$ $\because \because 00 \div \div \sim 0 \sim \sim$ $\therefore \because \because \dot{\sim} \dot{\sim} \dot{m}$

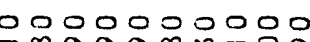
- 0 a a a n m- Da

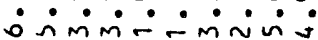

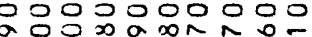
$\because \cdots \cdots$ ?

음으응ㅇㅇㅇㅇㅇㅇㅇㅢ $\dot{\sim} \dot{\sim} \dot{\sim} \dot{\sim} \cdot$.

옷옹ㅇㅁ웅응

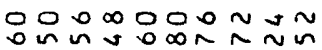

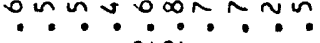

$x \sim \infty$ o

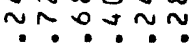

000000 $0.00,0.9$ ímiñ

UmamnNañ⿱

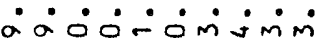

กmoง $0-0 \infty 0$ $\therefore \therefore$ i vivio in ก⿻上丨 $n$ íåni

nn $\infty 0-\operatorname{son} 00$

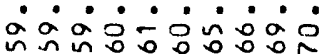

जOM N MOONa

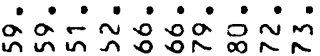

$a \infty 0 \pi-0$ ㅁํำํํำ

$\sim \sim a \infty$ o $n \sim J 50$

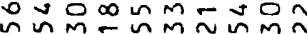
!.!. $! ? !$ $\bar{\sim} \bar{\sim} \bar{\sim} \bar{\sim} \bar{\sim} \bar{N} \bar{\sim}$ $\sim-\cdots+\cdots+\cdots$

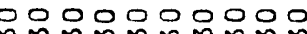

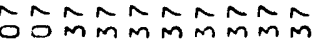
$\sim \sim \sim \sim \sim \sim \sim \sim \sim \sim$ $\infty \infty_{\infty} \infty \infty \infty \infty \infty \infty \infty$

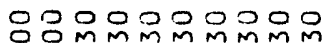

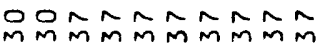
$\sim \sim \sim \sim N \sim N \sim N N$ $M M M M M M M M M M$

$\approx \tilde{N}= \pm \approx \pi \bar{N} \approx \sim$ $\tilde{z} \tilde{z} \tilde{z} \tilde{z} \tilde{z} \tilde{z} \tilde{z}$

111111111

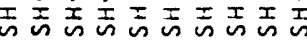
a a $a$ a $a$ a
nNOMNN-NOM

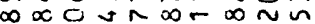
o orvno.onm $n$ V.V.V. $\infty \infty \bar{\sim} \bar{\sim}^{\infty} \bar{\sim} \bar{\sim} \bar{\sim}$ $-\sigma-\cdots+\infty-\cdots$

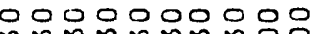

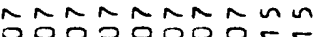

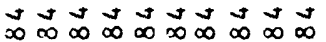

응은응음은은은 ตี coo. o o o o o o o MMMnMmM M M

$\sim a n a g a$

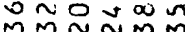
บ.!. $\bar{\sim} \bar{\sim} \bar{\sim} \bar{n}$

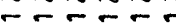

옹ㅇㅇ응응 nก⿻上แn

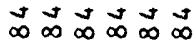

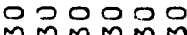
$\tilde{\sim} \tilde{\sim} \tilde{\sim} \tilde{ก} \tilde{N}$ o 00000

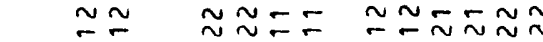

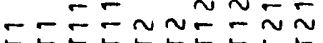

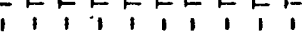

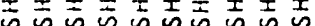
a a a a a a a a a $\bar{\sim} \tilde{N} \sim \tilde{N} \sim$

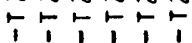
II工工贡贡贡 a a a a a $M M M m M$ 


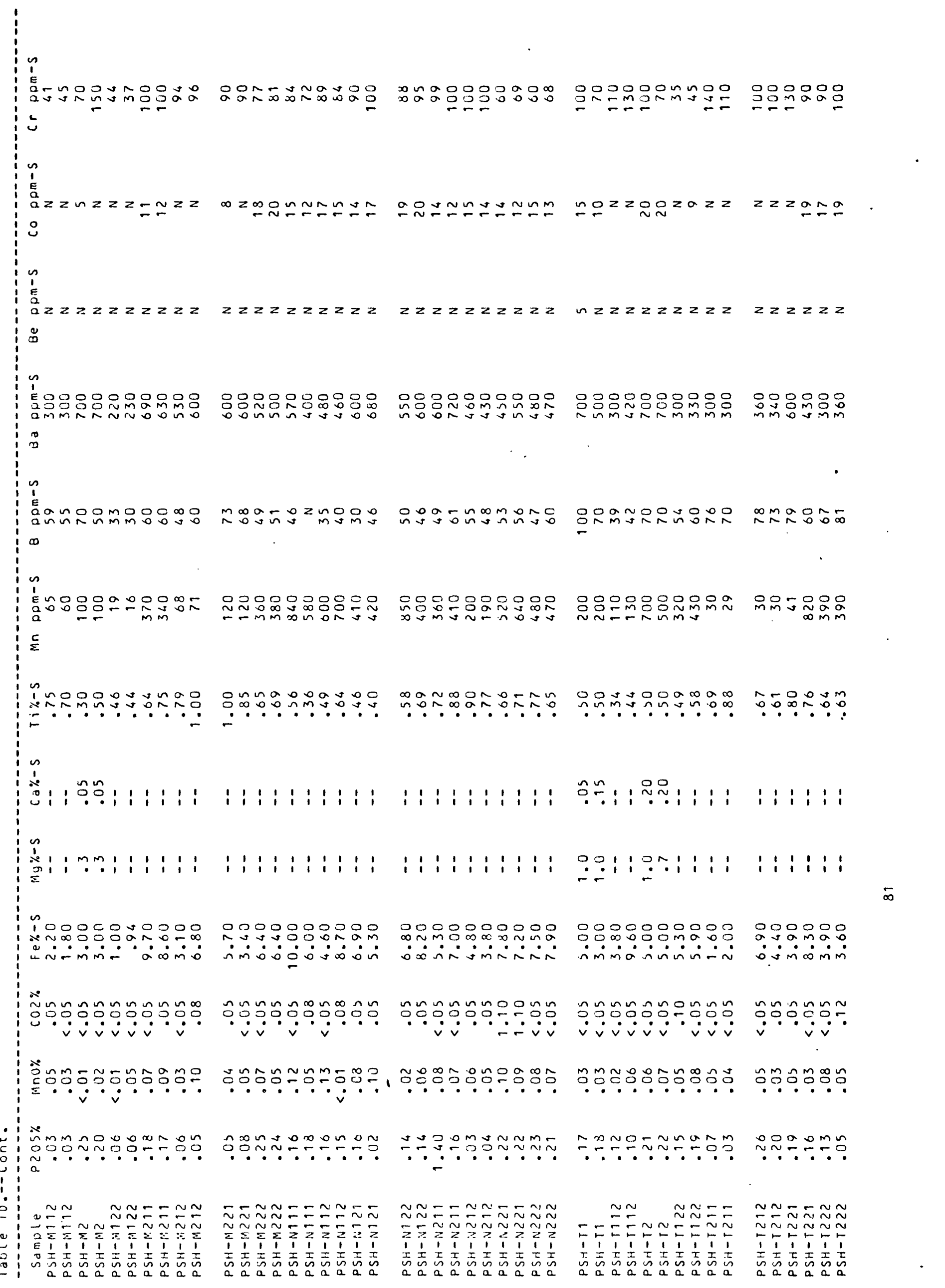




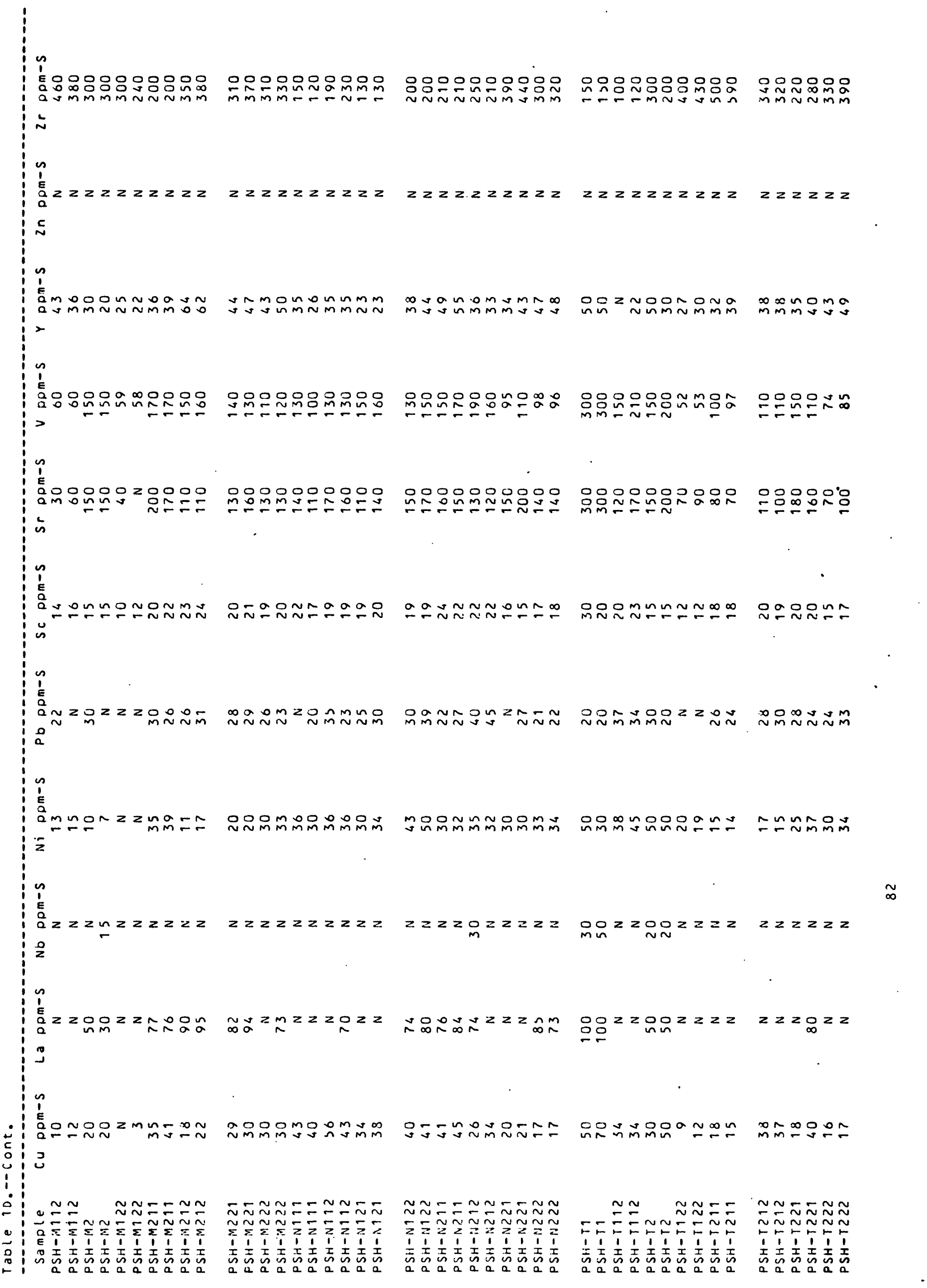




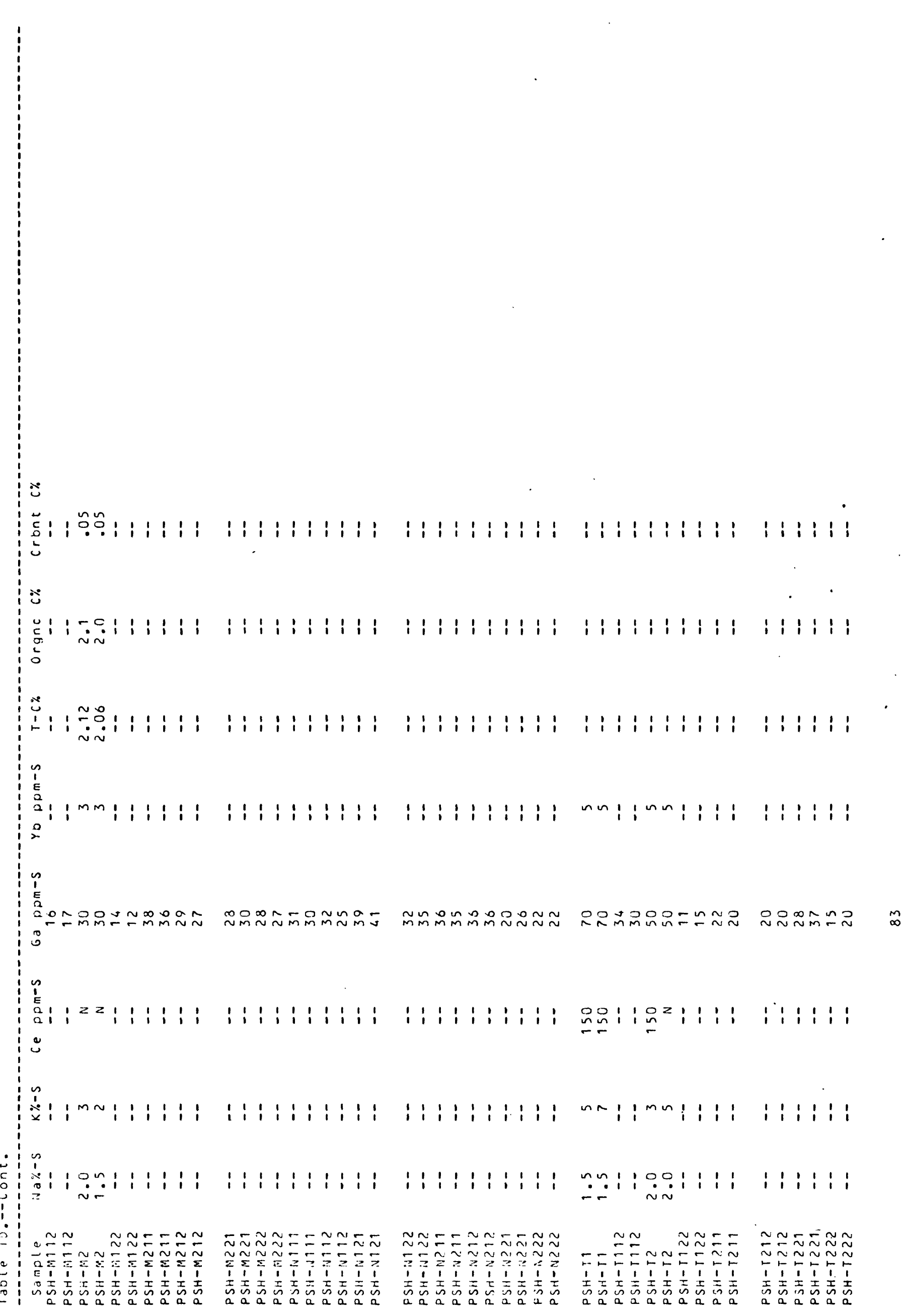


Table 2. --Elements commonly looked for, but rarely or never detected, by direct-reader emission spectrographic analysis, and their approximate lower limits of determination in parts per million.

Element Lower limit of determination

As

$A \cup$

$B$

Be

$B i$

cd

Ge

In

Mo

No

Po

Re

Sb

Sn

$T L$

w

Zn

$$
\begin{array}{r}
4 \\
20 \\
30 \\
5 \\
20 \\
\\
200 \\
100 \\
20 \\
20 \\
30 \\
10 \\
70 \\
300 \\
20 \\
50 \\
\\
500 \\
500
\end{array}
$$

84 
Taole 3.--Averaye modes for shale of Paleozoic age in kentucky. [C, Chat anooyde Onio and New Albany Shales; LM, Lower Mississippian; UM. Upper Mississipitan: $\mathbb{P}$. Pennsylvanian; ivumber of thin sections on which each mode is cased is shown in parentheses]

Framework grains silt Rock Felaspar
Watrix cement inuscovite Pyrite other 11

\section{Rock Unit (sand) fragments ?l}

Shale:

\begin{tabular}{|c|c|c|c|}
\hline$c$ & $(30)$ & $11 \%$ & \\
\hline Lin & $(32)$ & 10 & \\
\hline UiM & $(52)$ & 12 & 5 \\
\hline$P$ & $(02)$ & 17 & \\
\hline
\end{tabular}

$<1 \%$
1
4
3

$\begin{array}{lc}80 \% & 4 /<1 \% \\ 74 & 2 \\ 75 & <1 \\ 06 & 1\end{array}$

$<1 \%$
$<1$
$<1$
1

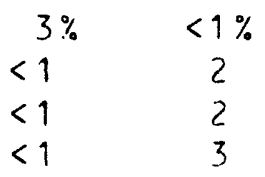

Sanuy shale:
Lin (o)

27

6

1

374122

$<1$

$<1$

5

Siliceous shale:
( 12$)$
31

13

14

10

7

2

$<1$

3.

$1 /$ Nostly hematite in C. LM and UM; both organic material dnd nematite in $\mathbb{R}$. 21 Mostly clay nellets with sutordinate phyllitic or schistose tragments. 3/ Incluces $3.2 \%$ Igsmanites.

4h komilic dolomite.

'Includes $0.9 \%$ fossil tragments. 
Table 4.4.--Sampling sites for rocks of the Chat tanooga, New Albany and ohio Shales. [See figure 2 for location of 7-1/2' quadrangles]

\begin{tabular}{|c|c|c|c|c|}
\hline Quadrangle & Sample No. & Sample Site & $\begin{array}{l}\text { Stratigraphic } \\
\text { Position }\end{array}$ & Remarks \\
\hline Briensburg & DSH-P 211 & $\begin{array}{l}2 \mathrm{kmE} \text { of Bethel } \\
\text { Church }\end{array}$ & $\begin{array}{l}6.1 \mathrm{~m} \text { below top of } \\
\text { Chattanooga }\end{array}$ & $\begin{array}{l}\text { Organic-free } \\
\text { (oxidized?) }\end{array}$ \\
\hline & DSH-P 212 & do & do & Organic-poor \\
\hline
\end{tabular}

Eddyville DSH-P111 SE edge of Vista $2.1 \mathrm{~m}$ below top of

Ridge Park (now Chat tanooga

under waters of

reservoir)

DSH-P112 do $1.8 \mathrm{~m}$ below top of weathered?

chat tanooga

Petroleum DSH-R111 In Trammel CK.. $4.6 \mathrm{~m}$ above base of Sandy

$2.7 \mathrm{~km} \mathrm{NW}$ of Maple Chat tanooga

Grove Church

DSH-R112 do C.1 m above base of Silty
Chat anooga

DSH-R121 $1.4 \mathrm{kmE}$ of Mt. At top of Splintery

Union School Chat tanooga

DSH-R122 do $1.5 \mathrm{~m}$ above base of Flinty

Chat tanooga

\begin{tabular}{|c|c|c|c|c|}
\hline \multirow[t]{4}{*}{ Aust in } & DSH-R211 & $\begin{array}{l}\text { E end of Long } \\
\text { Reach. barren } \\
\text { River valley }\end{array}$ & $\begin{array}{l}5.5 \mathrm{~m} \text { below top of } \\
\text { chattanooga }\end{array}$ & $\begin{array}{l}\text { silty, gypsum } \\
\text { coatings }\end{array}$ \\
\hline & OSH-R212 & do & $\begin{array}{l}0.6 \mathrm{~m} \text { below top of } \\
\text { Chattanooga }\end{array}$ & do \\
\hline & $O S H-R 221$ & $\begin{array}{l}2.2 \mathrm{~km} E \text { of } \\
\text { Maynard }\end{array}$ & $\begin{array}{l}4.6 \mathrm{~m} \text { below } \\
\text { chattanooga }\end{array}$ & Gypsum coatings \\
\hline & $D S H-R 222$ & do & $\begin{array}{l}3 \text { m below top of } \\
\text { chat tanooga }\end{array}$ & do \\
\hline
\end{tabular}


Table 4 A.-- Cont.

Quadrangle Samule No. Sample site
OSH-

Breeding

burksville

DSH-S 211

DSH-S212

$\checkmark S H-S 221$

USH-S 222

DSH-S111

do

do
In Strange Granch

$D S H-S 112$

DSH-S 121

DSH-S122

DSH-K111

DSH-K112

OSH-K 121

DSH-K122

DSH-K211

DSH-K212

DSH-K221

DSH-K2Z2
$2 \mathrm{~km} u p$ üarret

$1.6 \mathrm{~km} \mathrm{SE}$ of Howards jot tom School

ao

$0.8 \mathrm{~km} \mathrm{~N}$ of salem Churcn

do

$0.3 \mathrm{~km} \mathrm{~S}$ of Evans cemetery

Ci. $4 \mathrm{~km} \mathrm{~S}$ of Evans cemetery

U.8 km S of Neatsville

do

$2.8 \mathrm{~km}$ up Damron Ck.. h side

do Ck.. E side
Stratigraphic

Position
Remarks

$3 \mathrm{~m}$ above base of silty, gypsum Chat tanooga veins

$6.1 \mathrm{~m}$ above vase of do cnat t anooga

Pyritic, gypsum chat tanooya

$4.6 \mathrm{~m}$ above vase of paper shale, chat tanooga silty

2.1 m above Dase of Hematitic, chat anooga silty

$4.6 \mathrm{~m}$ above base of chat tanooga

$5.5 \mathrm{~m}$ above base of Pyritic, gyosum chat tanooga veins

$1.5 \mathrm{~m}$ above base of do Chat tanooga

3 m auove Dase of Cnat tanooga

do

$9.1 \mathrm{~m}$ above Dase of Paper shale Chat tanooga

$1.5 \mathrm{~m}(?)$ above base Dolomitic, of Chattanooga pyrite

$11 \mathrm{~m}$ above base of Pyritic Chat tanooga

$6.1 \mathrm{~m}$ above Dase of cnat tanonya

$1.5 \mathrm{~m}$ above base of do chat tanouya

$0.9 \mathrm{~m}$ bolow top of Chat tanouga

$1.8 \mathrm{~m}$ below top of

Chat tanooya 


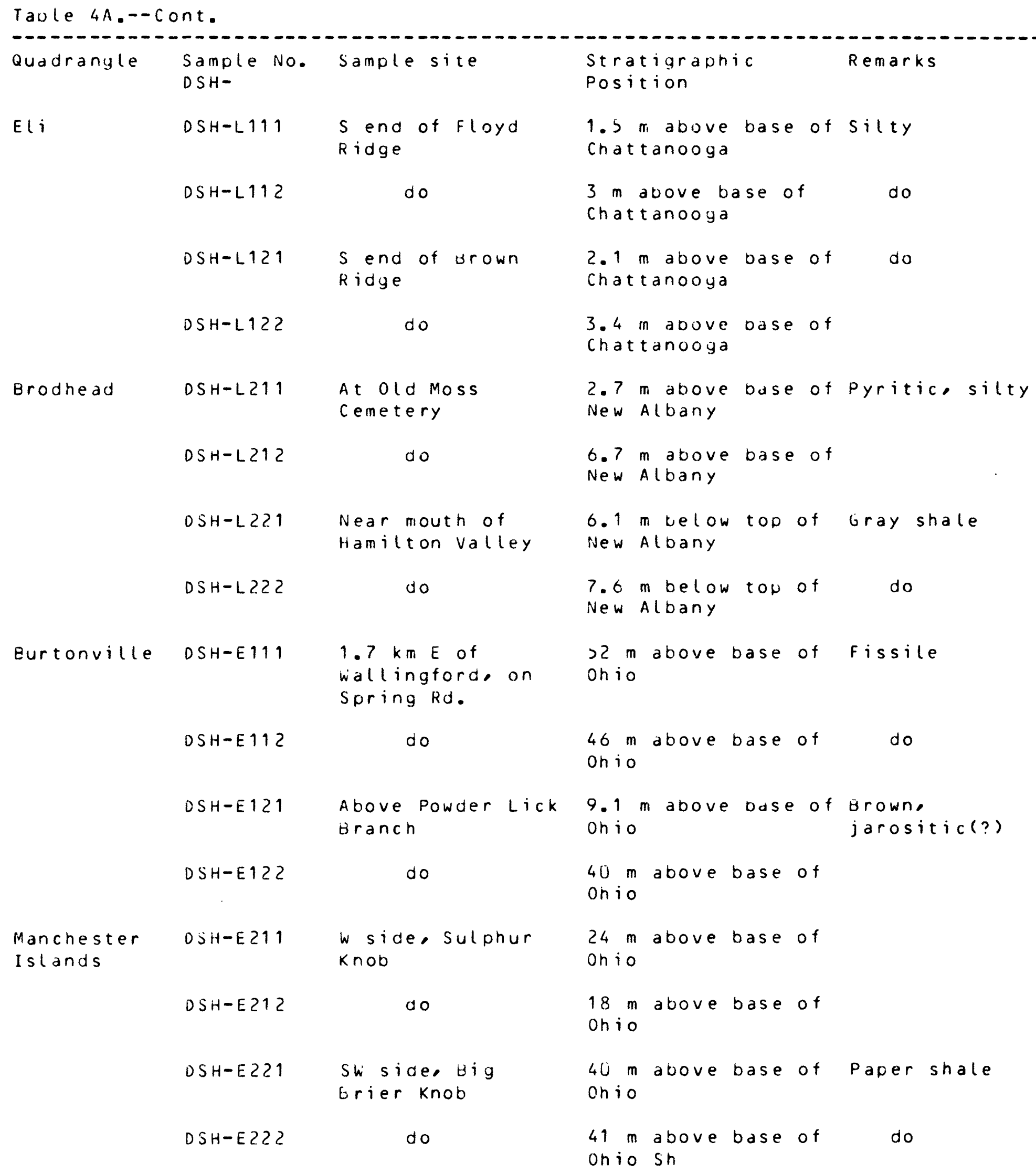


Table 43.--Sampling sites fur shole of Lower inississinpian age in Kentucky. [See fijure 2 for location of 7-1/2' quaurangles]

\begin{tabular}{|c|c|c|c|c|}
\hline Quadrangle & sample iro. & Sample site & $\begin{array}{l}\text { Stratigrawhic } \\
\text { Position }\end{array}$ & kemarks \\
\hline \multirow[t]{4}{*}{ Adolohus } & LAS HR 111 & $\begin{array}{l}1.2 \text { km ive of } \\
\text { Hinton Cemetery } \\
\text { in Little Trammel } \\
\text { Creek }\end{array}$ & $\begin{array}{l}35 \text { m above odse } \\
\text { of fort Payne }\end{array}$ & $\begin{array}{l}\text { Claystone, } \\
\text { weathered }\end{array}$ \\
\hline & LMSHR112 & do & $\begin{array}{l}12 \text { m above rase } \\
\text { of fort Dayne }\end{array}$ & Dolomite, cherty \\
\hline & L IA SHR 121 & $\begin{array}{l}\text { On road } E \text { of Oak } \\
\text { orove cnurch }\end{array}$ & $\begin{array}{l}\text { 2.3 mabove base } \\
\text { of Fort Payne }\end{array}$ & $\begin{array}{l}\text { Claystone, } \\
\text { we at hered'? }\end{array}$ \\
\hline & LMSHR 122 & do & $\begin{array}{l}\text { a m above oase } \\
\text { of furt ayne }\end{array}$ & Claystone \\
\hline \multirow[t]{4}{*}{ Holl and } & L43itk211 & $\begin{array}{l}1 \text { km SE of Mays- } \\
\text { ville School in } \\
\text { Rhoden Creek }\end{array}$ & $\begin{array}{l}4.6 \text { m above base } \\
\text { of fort bayne }\end{array}$ & $\begin{array}{l}\text { Claystone. } \\
\text { weathereu(?) }\end{array}$ \\
\hline & LinSth212 & do & $\begin{array}{l}\text { 1.J Ir aojue vase } \\
\text { of fort Dayne }\end{array}$ & Claystone \\
\hline & LMSHR2 21 & $\begin{array}{l}1.5 \mathrm{~km} \text { E of oak } \\
\text { Forest in Lony } \\
\text { Creek }\end{array}$ & $\begin{array}{l}\text { 4. } 6 \text { m doove base } \\
\text { of fort payne }\end{array}$ & jo \\
\hline & LMSHK222 & do & a.s & Jo \\
\hline \multirow[t]{4}{*}{ Ainaridaville } & LMSHS? 11 & $\begin{array}{l}\text { Z.x } \mathrm{km} N \text { of Sodck } \\
\text { Chapel }\end{array}$ & $\begin{array}{l}24 \text { m abuve base } \\
\text { of fort Payne }\end{array}$ & $\begin{array}{l}\text { Claystone, } \\
\text { silty }\end{array}$ \\
\hline & LMSHS212 & do & $\begin{array}{l}6.1 \text { II above Dase } \\
\text { of fort Jayne }\end{array}$ & $\begin{array}{l}\text { Claystone. } \\
\text { pyritic }\end{array}$ \\
\hline & LMSHS2 21 & $\begin{array}{l}\text { un } s \text { flank of } \\
\text { of collins arancn }\end{array}$ & $\begin{array}{l}34 \mathrm{~m} \text { above odse } \\
\text { of fort uyne }\end{array}$ & siltstone, sandy \\
\hline & LMSHSZ2? & do & $\begin{array}{l}9.1 \text { m above odse } \\
\text { of fort oayne }\end{array}$ & $\begin{array}{l}\text { Claystone, } \\
\text { black }\end{array}$ \\
\hline
\end{tabular}


Table 4..-- cont.

\begin{tabular}{|c|c|c|c|c|}
\hline Quadrangle & Sample No. & Sample site & $\begin{array}{l}\text { Stratigraphic } \\
\text { Position }\end{array}$ & Remarks \\
\hline \multirow[t]{4}{*}{$\begin{array}{l}\text { Wolf Creek } \\
\text { Dam }\end{array}$} & LMSHS111 & $\begin{array}{l}\text { At upper end of } \\
\text { Lick Creek }\end{array}$ & do & Claystone \\
\hline & LMSHS 112 & $\begin{array}{l}\text { Near ridgetope } \\
\text { S of Lick Creek }\end{array}$ & $\begin{array}{l}61 \mathrm{~m} \text { above base } \\
\text { of fort Payne }\end{array}$ & Siltstone, sandy \\
\hline & LMSHS121 & $\begin{array}{l}\text { On NE flank of } \\
\text { Billy Ridge }\end{array}$ & $\begin{array}{l}69 \mathrm{~m} \text { above base } \\
\text { of Fort Payne }\end{array}$ & do \\
\hline & LMSHS122 & do & $\begin{array}{l}65 \mathrm{~m} \text { above base } \\
\text { of Fort Payne }\end{array}$ & do \\
\hline \multirow[t]{4}{*}{ Knifely } & LMSHK111 & $0.8 \mathrm{~km} N$ of Purdy & $\begin{array}{l}78 \mathrm{~m} \text { above base } \\
\text { of fort Payne }\end{array}$ & do \\
\hline & LMSHK112 & do & $\begin{array}{l}81 \text { m above Dase } \\
\text { of Fort Payne }\end{array}$ & do \\
\hline & LMSHK1 21 & $\begin{array}{l}0.5 \mathrm{~km} \text { W of Bottom } \\
\text { District School }\end{array}$ & $\begin{array}{l}61 \mathrm{~m} \text { above oase } \\
\text { of fort Payne }\end{array}$ & Claystone, silty \\
\hline & LMSHK122 & $\begin{array}{l}0.7 \mathrm{~km} \text { w of Bottom } \\
\text { District School }\end{array}$ & $\begin{array}{l}43 \mathrm{~m} \text { above base } \\
\text { of Fort Payne }\end{array}$ & Claystone \\
\hline \multirow[t]{4}{*}{ Dunnville } & LINSHK211 & $\begin{array}{l}3.1 \mathrm{~km} \mathrm{NW} \text { of } \\
\text { Nillerfield School }\end{array}$ & $\begin{array}{l}34 \mathrm{~m} \text { above base } \\
\text { of Borden }\end{array}$ & Claystone, silty \\
\hline & LMSHK212 & do & $\begin{array}{l}6.1 \mathrm{~m} \text { above Dase } \\
\text { of Borden }\end{array}$ & Claystone \\
\hline & LMSHK221 & $\begin{array}{l}\text { L.9 } \mathrm{km} N \text { of Wilson } \\
\text { School }\end{array}$ & $\begin{array}{l}43 \mathrm{~m} \text { above base } \\
\text { of Borden }\end{array}$ & Claystone, silty \\
\hline & LMSHK222 & $\begin{array}{l}0.0 \mathrm{~km} N \text { of Wilson } \\
\text { School }\end{array}$ & $\begin{array}{l}64 \mathrm{~m} \text { above base } \\
\text { of 8orden }\end{array}$ & Caroonate, cherty \\
\hline \multirow[t]{4}{*}{ Eli } & LMSHL111 & $\begin{array}{l}2.4 \mathrm{~km} \mathrm{SE} \mathrm{of} \\
\text { Beasley School }\end{array}$ & $\begin{array}{l}2.4 \text { ni above base } \\
\text { of Fort Payne }\end{array}$ & Claystone \\
\hline & LMSHL112 & do & $\begin{array}{l}35 \mathrm{~m} \text { above base } \\
\text { of Fort Payne }\end{array}$ & Claystone, silty \\
\hline & LMSHL121 & $\begin{array}{l}\text { U.4 km S of New } \\
\text { Pt. Pleasant Ch. }\end{array}$ & $\begin{array}{l}30 \mathrm{~m} \text { above base } \\
\text { of Fort Payne }\end{array}$ & $\begin{array}{l}\text { Claystone. } \\
\text { chertye pyritic }\end{array}$ \\
\hline & LMSHL1 22 & do & $\begin{array}{l}27 \text { m above base } \\
\text { of Fort Payne }\end{array}$ & Claystone \\
\hline
\end{tabular}


Table 4C.--Sampling sites for shale of Upper Mississippian age. [See tigure ? for location of 7-1/2' quadrangles]

\begin{tabular}{|c|c|c|c|c|}
\hline Quadrangle & Sample No. & Sample site & $\begin{array}{l}\text { Stratigraphic } \\
\text { Position }\end{array}$ & Remarks \\
\hline \multirow[t]{4}{*}{ Shet lerville } & UMSHH111 & $\begin{array}{l}0.4 \mathrm{~km} w \text { of } \\
\text { Carrsville }\end{array}$ & $\begin{array}{l}3 \text { m below top of } \\
\text { Kincaid }\end{array}$ & Claystone \\
\hline & UMSHH 112 & $\begin{array}{l}0.2 \mathrm{~km} w \text { of } \\
\text { carrsville }\end{array}$ & $\begin{array}{l}1 \text { m below top of } \\
\text { chlore ls }\end{array}$ & Claystone, silty \\
\hline & UMSHH 121 & $\begin{array}{l}1.6 \mathrm{~km} \mathrm{~s} \text { of } \\
\text { Carrsville }\end{array}$ & $\begin{array}{l}3 \text { m above base of } \\
\text { Golconda }\end{array}$ & $\begin{array}{l}\text { Claystone, } \\
\text { fossiliferous }\end{array}$ \\
\hline & UMSHH122 & do & do & do \\
\hline \multirow[t]{4}{*}{ Rosiclare } & UMSHH211 & $\begin{array}{l}4.2 \mathrm{~km} S E \text { of } \\
\text { Carrsville }\end{array}$ & $\begin{array}{l}3 \text { m below top of } \\
\text { Renault }\end{array}$ & Claystone \\
\hline & UMSHH 212 & do & do & do \\
\hline & UMSHH 221 & $\begin{array}{l}0.8 \mathrm{~km} E \text { of } \\
\text { Carrsville }\end{array}$ & $\begin{array}{l}\text { At top of } \\
\text { Paint Creek }\end{array}$ & do \\
\hline & UMSHH 222 & do & do & $\begin{array}{l}\text { Claystone, } \\
\text { carbonaceous }\end{array}$ \\
\hline \multirow[t]{4}{*}{ Honey Grove } & UNSHQ 111 & $\begin{array}{l}\text { At Pleasant Hill } \\
\text { Church }\end{array}$ & $\begin{array}{l}\text { At base of } \\
\text { Waltersourg }\end{array}$ & do \\
\hline & UNSHQ112 & do & $\begin{array}{l}3 \text { m above base of } \\
\text { waltersourg }\end{array}$ & do \\
\hline & UMSHQ121 & At Britmart & $\begin{array}{l}15 \mathrm{~m} \text { above base of } \\
\text { Big Clifty Sandstone } \\
\text { Member (Golconda) }\end{array}$ & Claystone \\
\hline & UMSHQ12? & do & $\begin{array}{l}18 \mathrm{~m} \text { aoove base of } \\
\text { Big Clifty Sandstone } \\
\text { Member (Golconda) }\end{array}$ & Soil \\
\hline \multirow[t]{4}{*}{$\begin{array}{l}\text { Pleasant } \\
\text { Green Hill }\end{array}$} & UMSHQ 211 & $\begin{array}{l}2.7 \mathrm{~km} S E \text { of } \\
\text { Palestine Church }\end{array}$ & $\begin{array}{l}7.6 \mathrm{~m} \text { above base of } \\
\text { Hardinsuurg }\end{array}$ & $\begin{array}{l}\text { Claystone. } \\
\text { fossiliferous }\end{array}$ \\
\hline & UMSHQ212 & do & $\begin{array}{l}12 \text { m above base of } \\
\text { Hardinsburg }\end{array}$ & Claystone \\
\hline & UMSHQ221 & $\begin{array}{l}0.4 \mathrm{~km} \mathrm{~N} \text { of boyd } \\
\text { Cemetery }\end{array}$ & $\begin{array}{l}7.6 \text { m above base of } \\
\text { Tar Springs }\end{array}$ & $\begin{array}{l}\text { Shale, sandy, } \\
\text { weatnerea }\end{array}$ \\
\hline & UMSHQ222 & do & do & do \\
\hline
\end{tabular}


Taule 4 c.- cont.

--- - - - - - -

Quadranjle

Sample No.

Sample site

Stratigraphic

Reinarks

position

South union

UMSHR111 $1.1 \mathrm{~km}$ iv of Felts
Cemetery

$3 \mathrm{~m}$ below top of jig $\mathrm{Cl} i f t y$

Sanastone Memuer

(Golconda)

UNSHK112

$0.8 \mathrm{~km}$ NE of Felts $7.1 \mathrm{~m}$ aDove ouse of

Claystone.

sanoy

cemetery

Jig Clitty Sancstone

inember (Golconara)

JPSHRT21 at head of

Eperson hollow

7.6 m above base of

uig $\mathrm{Cli}$ it

sandstone pember

(Golconoa)

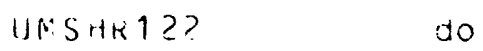

a)

Ruckfielo

UNSHR? 11

C..7 km E of 3 lue Level

3iu lifty

Sandstone memuer

(Golconda)

UWSHK212

do

2 in thelow top of

Girkin

UASHR?? 1

1). $5 \mathrm{~km} E$ of Cedar Grove Church

UNSHR22?

do

At top of Girkin

$0.1 \mathrm{~m}$ doove Dase of

Biy Clifty Sanastone

Member (iolconá)

Nillerstown

UNSHJ 111

$1.7 \mathrm{~km} N$ of Little

flock Church

At base(?) of Hiy

Clifty Sandsturie

Member (Golconua)

Claystone.

fossiliterous

UNSHJ112

40

12 $m(?)$ above dase

Claystone, silty

of Biy Clifty

Sandstone remuer

(úlcuna)

$\operatorname{JSHJ121}$

$0.2 \mathrm{~km} \mathrm{siw}$ of Lacon

3 m auove base of

inember (bolconoa)

Uitsid $12 ?$

do claystone

Claystone,

sandy, weathered

soil

Claystone

do

do

llaystone.

silty, weathered

o ó in avove base of

ilg (lifty sanastone

hemoer (Golcunaa) 


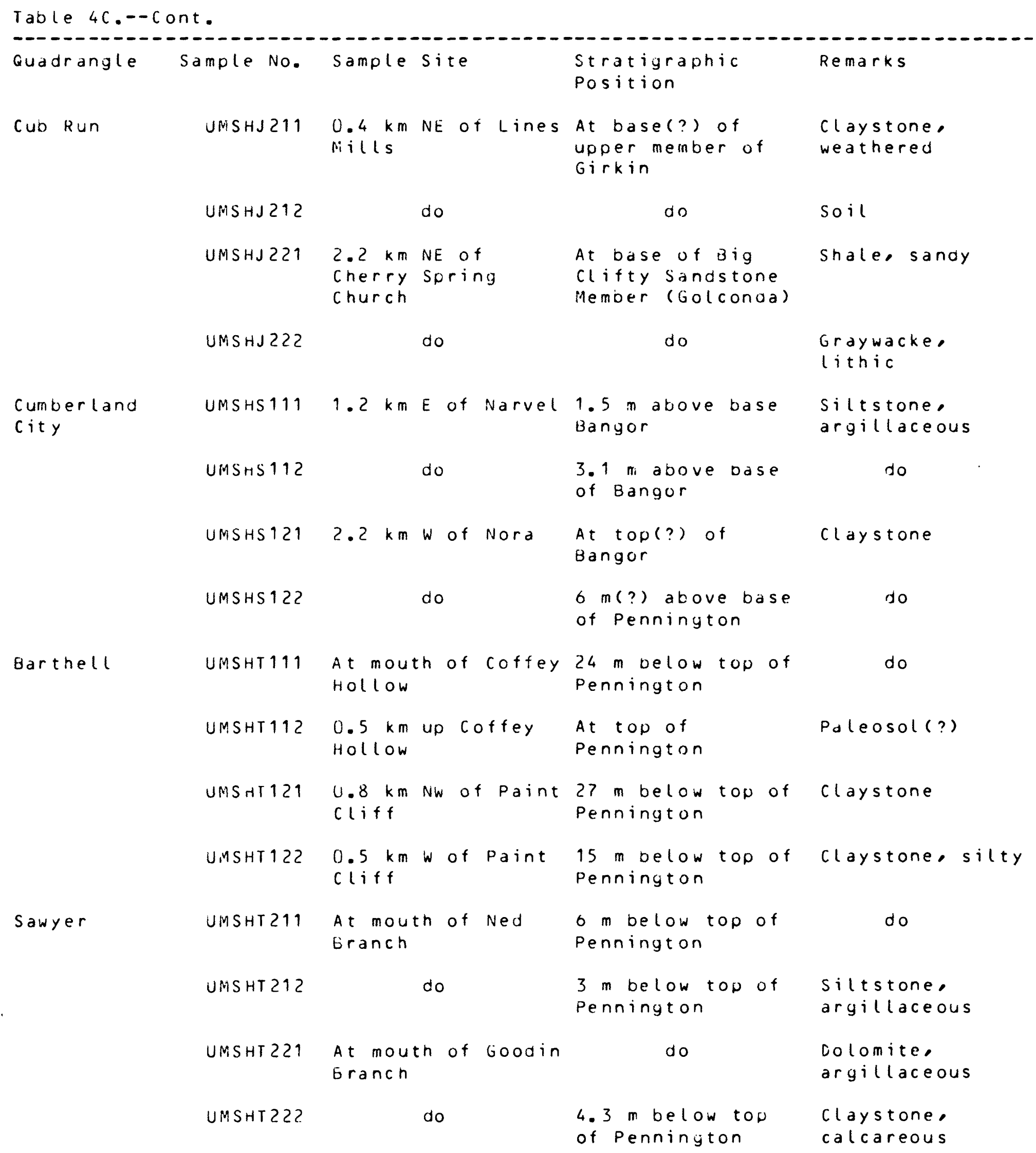

Siltstone, argillaceous

do

Claystone

do

do

Poleosol(?)

Claystone

claystone, silty

do

Siltstone, argillaceous

Colomite, argillaceous

Claystone, calcareous 
Table 4 C.-- Cont.

Quadrangle Sainle No. Sample site

Maretouris

Eisnill

hrijley

Portsingen Cave uranch

\author{
UMSHL111 Below Backoone \\ hiage
}

JMSIL 112

JPSHL 121

$1.3 \mathrm{~km}$ NE of Freedom School

Jisht.15?

UNSHL ? 11

L. E $k m$ w of norill

UNSIL 212 U.8 km NW of Piorill

JVSHL? ? 1

$0.2 \mathrm{~km} \mathrm{Nw}$ of ishill school

UWSHL 222

do

JUSHE 211

In quarrye at inouth of oakley

UN SHE 212

JMSHE 221

UNSHE 2??

UNSHE111

JMSHE112

U以SUE 121

U.S SHE 122 do

At Leisure

do

At head of siloan uranch

do

In quarry, $1.9 \mathrm{k}$ : iv of valley view School

do
Stratigrapnic

position

7 in (?) Delow top of Pennington

do

do

1.5 m velow top of Claystone Pennington

At tcp of

Penninytion

co

$9.1 \mathrm{~m}$ Delow ton of Soil

Newinan

$y$ in (?) above uase of Pennington

5.? m above vase

of Pennington

do

3.? m above base

of Pennington

1? $m(?)$ oelow top soil

of Pennington(?)

$10 m(?)$ oelow top

of Pennington(?)

Claystone, sandy

4.5 in delow top of Claystone, silty Pennington(?)

6 m delus top of

Pennington(?)

Siltstone. argillaceous

\section{At top of}

Pennington

(1)

Claystone. sandy, we atnerea

Claystone. we athered

3.5 mi(?) below top Claystone, rennington colomitic, we athered

6 melow top uf Paleosol(?) Dennington 
Table 40.--Sampling sites for shale of Pennsylvanian age. [See figure 2 for location of $7-1 / 2^{\prime}$ quadrangles]

Quadrangle Sample No. Sample Site $\begin{array}{ll}\text { Shetlerville PSH-H211 } & 2.7 \mathrm{~km} \text { W of } \\ & \text { Carrsville }\end{array}$

\begin{tabular}{ll} 
PSH-H212 & \multicolumn{1}{c}{ do } \\
PSH-H221 & $\begin{array}{l}1.4 \text { km W of } \\
\text { Carrsville }\end{array}$ \\
PSH-H222 & $\begin{array}{l}1.3 \text { km W of } \\
\text { Carrsville }\end{array}$
\end{tabular}

Sal em

PSH-H111

$0.2 \mathrm{~km} \mathrm{~S}$ of Glendale Church

PSH-H112

do

PSH-H 121

$0.2 \mathrm{~km} \mathrm{~s}$ of Corn School

PSH-H122

do

Slaughters

PSH-I 211

$0.5 \mathrm{~km} \mathrm{SW}$ of

Bailey Cemetery

PSH-1212

$0.3 \mathrm{~km} \mathrm{SW}$ of

Bailey cemetery

PSH-I 221

$0.7 \mathrm{~km} \mathrm{NW}$ of

Slaughters

PSH-I 222

do

Stratigraphic

Position

5 m above base of Grinastaff Sanast one Meinber

( $T$ radewater)

do

do

3 m below base of Graywacke Gentry coal

(Caseyville)

3 m above top of

Gentry Coal

(Caseyville)

$23 \mathrm{~m}$ above base of Graywacke, Caseyville carbonaceous

$50 \mathrm{~m}$ above base of Graywacke caseyville

$60 \mathrm{~m}(?)$ above tase Sandstone, silty of Caseyville

do

Siltstone, sandy, weathered(?)

$320 \mathrm{~m}$ above base of Claystone No. 9 Coal

(Breathit t)

$31 \mathrm{~s} m$ above Dase of do No. 9 Coal (Breathit t)

$330 \mathrm{~m}$ above base of Siltstone, No. 9 Coal (Breathit t) weathered

do

Claystone 
Table $40 .--\operatorname{cont}$.

Quadrangle Sample wo. Sample Site

Stratigrawhic

position

1) m velow Lead Creek Limestune of Crider (1913)

$$
\text { PsH-J111 } 1 \mathrm{~km} \text { iNh of Adair }
$$

do

do

$$
P S H-J 2
$$$$
\text { (i. } 8 \mathrm{~km} \mathrm{~S} \text { of }
$$$$
\text { Grove Church }
$$$$
\text { PSH-J122 } 1 \mathrm{~km} \mathrm{~s} \text { of poplar }
$$$$
\text { Grove Church }
$$

do

coal (Tradewater)

$12 \mathrm{~m}$ above base ot

Caseyville
PSH-J211 1.J km $\mathrm{m}$ of i rownsville

$$
\text { PSII-J21? }
$$

do

$$
\begin{array}{ll}
\text { PSH-J } 221 & 1.2 \mathrm{~km} \mathrm{~S} \text { of } \\
& \text { Lindsayville }
\end{array}
$$
$13 \mathrm{~m}$ ature base of caseyville
3 m above base of Caseyville

un

$$
\text { PSH-J222 }
$$

do
PSH-T 211
At head of Dutch uranch

At ton ot shale He nber $O$ (LeE)

PSH-T $21 \geq \quad$ do

$6 \mathrm{~m}$ below top of Shale vember i) (Lee)
PSH-T221 1.2 km is of áald- At top of Shale rock
Member H (Lee)
Psit-T222 $1 \mathrm{~km}$ is of taddrock $3 \mathrm{~m}$ below ton of Shale Veinber ti (Lee)

Siltstone, sandy

Siltstone, sandy

Claystone

Remarks

siltstone. argillaceous

Claystone. wyritic

Claystone, silty sandstone, silty

Claystone, silty,

carbonaceous

do

Claystone, silty stained, silty

Claystone. carbonaceous

claystone, silty
Claystone, iron- 
Table 40.-- cont

Quadrangle Sample No. Sample Site

Vox

London

Parrot

$$
\begin{array}{ll}
\text { PSH-T1 } & \begin{array}{l}
1.3 \mathrm{kin} E \text { of } \\
\text { Corinth School }
\end{array} \\
\text { PSH-T112 } & \begin{array}{l}
1 \mathrm{~km} \text { E of Corinth } \\
\text { School }
\end{array} \\
\text { PSH-T2 } & \begin{array}{l}
1.4 \mathrm{~km} \mathrm{NW} \text { of } \\
\text { barton Chapel }
\end{array} \\
\text { PSH-T122 } & \begin{array}{l}
1.5 \mathrm{~km} N W \text { of } \\
\text { Barton Chapel }
\end{array}
\end{array}
$$

PSH-L1 $0.9 \mathrm{~km} \mathrm{NE}$ of Macedonia School

PSH-L 112

do

$$
\begin{aligned}
& \text { PSH-L2 } 0.8 \mathrm{~km} \mathrm{NW} \text { of } \\
& \text { McWhorter }
\end{aligned}
$$

PSH-L122 $1 \mathrm{~km} \mathrm{~N}$ of Micwhorter

$$
\begin{array}{ll}
\text { PSH-L211 } & 1.6 \mathrm{~km} \text { SW of } \\
& \text { Dabolt } \\
\text { PSH-L212 } & 1.9 \mathrm{~km} \mathrm{SW} \text { of }
\end{array}
$$

$$
\begin{aligned}
& \text { PSH-L221 } 0.5 \mathrm{~km} \mathrm{NW} \text { of } \\
& \text { Cornet te } \\
& \text { PSH-L22? } 0.6 \mathrm{~km} \mathrm{wof} \\
& \text { cornet te }
\end{aligned}
$$

Stratigraphic

Position

$1 \mathrm{~m}$ above blue Gem Claystone

Coal (óreathitt)

$30 \mathrm{~m}$ below Blue Gem

Coal (Breathitt)

Claystone, weathered

$3 \mathrm{~m}$ below base of Corbin Sandstone

Member (Lee)

$10 \mathrm{~m}$ velow base of sandstone, silty Corbin Sandstone Member (Lee)

$35 \mathrm{~m}$ above Lily Coal (Breathitt)

Siltstone, sandy

do

do

$16 \mathrm{~m}$ below horizon(?) of Lily Coal (Breathitt)

On horizon(?) of Lily Coal (Breathitt)

Claystone, silty

$100 \mathrm{~m}(?)$ above top Claystone

of Pennington

(c-zone soil?)

$110 \mathrm{~m}(?)$ above top siltstone, of Pennington

argillaceous,

carbonaceous

$70 \mathrm{~m}($ ?) above top

of Penninyton

Claystone, sandy

$100 \mathrm{~m}(?)$ above top

Claystone

of Pennington 
$\operatorname{Tan} L=4 D_{0}--\operatorname{con} t$.

Guadranjle Samile ivo. Sample site

Tintop

Tintop

white id

Lancer$$
\text { PSH-M21? }
$$$$
\text { do }
$$$$
\text { PSH-it } 221
$$$$
\text { PSH-M 22? }
$$$$
\text { Evanston }
$$

$$
\text { PSH-i+112 }
$$

$$
\begin{aligned}
& \text { PSH-n2 } 1 \mathrm{~km} \mathrm{~s} \text { of } \\
& \text { bloomingt on } \\
& \begin{aligned}
& \text { Psh-i12? } \text { is km s of } \\
& \text { wloomington }
\end{aligned}
\end{aligned}
$$

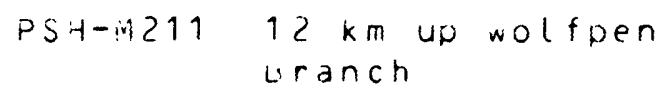

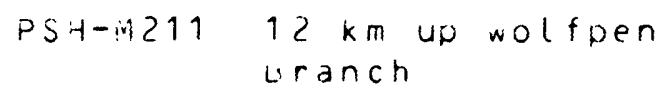$$
\begin{aligned}
& 1.9 \text { KIII NE of } \\
& \text { Evanston }
\end{aligned}
$$$$
\text { 2. } 2 \mathrm{~km} \text { iNE of }
$$

PSH-.11 H. $3 \mathrm{~km} \mathrm{Nw}$ of fock
HOUSE Scnool$$
1.5 \mathrm{~km} \mathrm{~N} \text { of } \mathrm{kock}
$$$$
\text { House School }
$$
PS:A-iv211 $0.7 \mathrm{~km}$ Siw of Dewey $15 \mathrm{~m}$ below base of uam Magoftin inemoer (Breathit $)$
At oase(?) of Mayoftin Mienoer (sreathit t)

Stratiyrawhic position

kemarks

2 m aoove base of Mayoftin Memoer (breatnit $)$

Claystone, carbonaceous

At top(?) of Feach Orchara coal Lone (Breathit )

$7 \mathrm{~m}$ above prater coal (isreathitt)

siltstone, coaly

At ton(?) of fire

Clay Coal

(isceathitt)

Siltstone.

caroonaceous

Claystone

5 (?) above fire

Clay coal

(areathite)

Claystone. iron-stainea

Siltstone. sandy, coaly

3 melow base of indyoffin inemoer (breathit t)

do

15 m below fire Clay Coal

(ireatnit $)$

Graywacke

Claystone. carbonaceous

$\begin{array}{ll}\text { PSH-N212 } & \begin{array}{l}\text { i. } k \text { km SW of Dewey } \\ \text { i.am }\end{array} \\ \text { PSH-W221 } & \begin{array}{l}0.5 \mathrm{~km} N W \text { of ilayo } \\ \text { Cemetery }\end{array}\end{array}$

At ton(?) of fire Clay kider Coal (ureathit )

do
At top(?) of viner Elkhorn do. 3 Coal (sreatnit $t$ )

$$
\begin{aligned}
& \text { PJinz?2 U.3 km in of mayo } \\
& \text { cemetery }
\end{aligned}
$$

5 in auove Jpier Elkhorn ivo. $3 \mathrm{COal}$ (sreatnit t)
Siltstone. argillaceous

Siltstone. carbonaceous, weathereo(?) 


\begin{tabular}{|c|c|c|c|c|c|}
\hline Quadranyle & Sample No. & Sample & Site & $\begin{array}{l}\text { Stratigraphic } \\
\text { Position }\end{array}$ & Remarks \\
\hline \multirow[t]{4}{*}{ Matewan } & PSH-N 111 & $\begin{array}{l}0.6 \mathrm{~km} \\
\text { Christ } \\
\text { church }\end{array}$ & $\begin{array}{l}\text { SE of } \\
\text { Temple }\end{array}$ & $\begin{array}{l}3 \text { m above Lower } \\
\text { Thacker coal } \\
\text { (Breathitt) }\end{array}$ & Claystone \\
\hline & PSH-N 112 & $\begin{array}{l}0.8 \mathrm{~km} \\
\text { christ } \\
\text { Church }\end{array}$ & $\begin{array}{l}\text { SE of } \\
\text { Temple }\end{array}$ & $\begin{array}{l}\text { At top of Lower } \\
\text { Thacker coal } \\
\text { (Breathitt) }\end{array}$ & $\begin{array}{l}\text { Siltstone, } \\
\text { argillaceous }\end{array}$ \\
\hline & PSH-N 121 & $\begin{array}{l}\text { At head } \\
\text { Fork }\end{array}$ & of May & $\begin{array}{l}\text { At top(?) of Hamlin } \\
\text { Coal (Breathitt) }\end{array}$ & $\begin{array}{l}\text { Claystone, } \\
\text { carbonaceous }\end{array}$ \\
\hline & $P S H-N 122$ & & do & $\begin{array}{l}12 \text { m above } \\
\text { williamson coal } \\
\text { (Breathit })\end{array}$ & $\begin{array}{l}\text { Claystone, } \\
\text { carbonaceous, } \\
\text { weathered(?) }\end{array}$ \\
\hline \multirow[t]{4}{*}{ wrigley } & PSH-E 1 & $\begin{array}{l}3.1 \mathrm{~km} \\
m i l \mathrm{ls}\end{array}$ & w of Blairs & $\begin{array}{l}27 \text { m above base of } \\
\text { Breathitt }\end{array}$ & Claystone \\
\hline & PSH-E 112 & & do & $\begin{array}{l}26 \mathrm{~m} \text { above base of } \\
\text { Breathitt }\end{array}$ & $\begin{array}{l}\text { Claystone, } \\
\text { black }\end{array}$ \\
\hline & PSH-E 2 & $\begin{array}{l}1.1 \mathrm{~km} \\
\text { wrigley }\end{array}$ & NW of & $\begin{array}{l}9 \text { m above base of } \\
\text { Breatnitt }\end{array}$ & $\begin{array}{l}\text { Siltstone, } \\
\text { carbonaceous }\end{array}$ \\
\hline & $P S H-E 122$ & & do & $\begin{array}{l}\text { At base(?) of } \\
\text { Breathitt }\end{array}$ & $\begin{array}{l}\text { Claystone, } \\
\text { black }\end{array}$ \\
\hline \multirow[t]{4}{*}{ Haldeman } & PSH-E211 & $\begin{array}{l}0.8 \mathrm{~km} \\
\text { christi }\end{array}$ & $\begin{array}{l}\text { Nw of } \\
\text { ian cenetery }\end{array}$ & $\begin{array}{l}3 \text { m above top of } \\
\text { olive Hill Clay } \\
\text { Bed of Crider (1913) }\end{array}$ & $\begin{array}{l}\text { Siltstone, } \\
\text { argillaceous }\end{array}$ \\
\hline & PSH-E212 & $\begin{array}{l}1 \mathrm{~km} \mathrm{NW} \\
\text { Christi }\end{array}$ & $\begin{array}{l}\text { of } \\
\text { ian Cemetery }\end{array}$ & $\begin{array}{l}\text { From Olive Hill } \\
\text { Clay oed of Crider } \\
\text { (1913) }\end{array}$ & $\begin{array}{l}\text { Claystone, } \\
\text { fossiliferous, } \\
\text { carbonaceous, } \\
\text { pyritic }\end{array}$ \\
\hline & PSH-E 221 & $\begin{array}{l}1.7 \mathrm{~km} \\
\text { school }\end{array}$ & S of ditney & $\begin{array}{l}30 \mathrm{~m} \text { dbove base of } \\
\text { Breathitt }\end{array}$ & Claystone, silty \\
\hline & PSH-E 222 & $\begin{array}{l}1.5 \mathrm{~km} \\
\text { school }\end{array}$ & S of oitney & do & Claystone \\
\hline
\end{tabular}


Table 4D.--cont.

\begin{tabular}{|c|c|c|c|c|}
\hline Quadrangle & Sample No. & Sample site & $\begin{array}{l}\text { Stratigraphic } \\
\text { Position }\end{array}$ & Remarks \\
\hline \multirow[t]{4}{*}{ Rush } & $P S H-F 1$ & $\begin{array}{l}\text { At head of upper } \\
\text { Stinson Creek }\end{array}$ & $\begin{array}{l}7 \text { m doove Princess } \\
\text { No. } 7 \text { Coal } \\
\text { (Breathitt) }\end{array}$ & Siltstone, sandy \\
\hline & PSH-F112 & $\begin{array}{l}1 \mathrm{~km} \text { in of williams } \\
\text { Creek }\end{array}$ & $\begin{array}{l}\text { At top(?) of } \\
\text { Princess No. } 7 \\
\text { Coat (sreathitt) }\end{array}$ & $\begin{array}{l}\text { siltstone, } \\
\text { argillaceous }\end{array}$ \\
\hline & $P S H-F 2$ & $\begin{array}{l}\text { U. } 7 \mathrm{~km} \text { Siw of } \\
\text { Denton }\end{array}$ & $\begin{array}{l}10 \mathrm{~m} \text { auove Princess } \\
\text { No. } 7 \text { Coal } \\
\text { (Breathitt) }\end{array}$ & Claystone, silty \\
\hline & OSH-F 122 & $\begin{array}{l}1 k m \text { w of venton } \\
\text { in } k \pi \text { cut }\end{array}$ & $\begin{array}{l}\text { Weneath Princess } \\
\text { No. } 3(?) \text { cod } \\
\text { (areathit })\end{array}$ & $\begin{array}{l}\text { Claystone, } \\
\text { fossiliferous, } \\
\text { carvonaceous, } \\
\text { sandy }\end{array}$ \\
\hline \multirow[t]{4}{*}{ boltsfork } & $P S H-F 211$ & $\begin{array}{l}\text { At head of } \\
\text { friendshin creek }\end{array}$ & $\begin{array}{l}\text { 1s m above Ames(?) } \\
\text { Limestone vember } \\
\text { (conemaugh) }\end{array}$ & $\begin{array}{l}\text { Claystone } \\
(\text { Paleosol?) }\end{array}$ \\
\hline & $P S H-F 21 ?$ & do & $\begin{array}{l}26 \mathrm{~m} \text { above Ames(?) } \\
\text { Limestone felluer } \\
(\text { Conemaugh) }\end{array}$ & do \\
\hline & $P S H-F 221$ & $1.5 \mathrm{~km}$ is of Garner & $\begin{array}{l}12 \text { m below tow of } \\
\text { areathitt }\end{array}$ & claystone \\
\hline & $P S H-F 22 ?$ & co & $\begin{array}{l}1 \mathrm{~m} \text { above Princess } \\
\text { Ho. } 7 \text { Codl } \\
\text { (ureatnit })\end{array}$ & do \\
\hline
\end{tabular}


Table 5A.-- Components of geochemical variance for rocks of the organic-rich Chat tanooya, New Altany, and uhio Shales in kentucky. [Components given as percentages of total logaritnmic variance. Total $V(\log x)$ : , component significantly different from zero at the 0.05 prodability level: see text for explanation of $v(m)]$

\begin{tabular}{|c|c|c|c|c|c|c|c|}
\hline & $\begin{array}{l}\text { Total } \\
v(\operatorname{Loy} x)\end{array}$ & $\begin{array}{l}\text { Hetween } \\
\text { Areas } \\
V(A)\end{array}$ & $\begin{array}{l}\text { between } \\
\text { Quads } \\
V(B)\end{array}$ & $\begin{array}{l}\text { between } \\
\text { Localities } \\
V(C)\end{array}$ & $\begin{array}{l}\text { between } \\
\text { Samples } \\
V(D)\end{array}$ & $\begin{array}{l}\text { Between } \\
\text { Replicates } \\
V(D)\end{array}$ & $v(m) 1 /$ \\
\hline \multicolumn{8}{|l|}{$0 x i$ des: } \\
\hline sioz 21 & 41.6 & $14 \%$ & $32 \% \star$ & $21 \%$ & $33 \% \star$ & $<1$ & $<1.0$ \\
\hline$A\left(203^{\circ}\right.$ & .0038 & 17 & $<1$ & 31 & $51 *$ & 1 & $<1.0$ \\
\hline$m_{y} 0$ & .0276 & 29 & $20 \star$ & 14 & $28 \star$ & 9 & $<1.0$ \\
\hline CaO & .2192 & $15 *$ & $<1$ & 22 & $36 *$ & 25 & $<1.0$ \\
\hline NaZU & .0664 & 31 & $13 *$ & $11 \star$ & $<1$ & 45 & $<1.0$ \\
\hline$k 20$ & .0047 & 2 & $<1$ & 29 & $46 *$ & 23 & $<1.0$ \\
\hline $\mathrm{H} 20+$ & .0290 & $<1$ & $<1$ & 2 & 24 & 75 & $<1.0$ \\
\hline Ti02 & .0040 & $<1$ & $<1$ & 25 & $52 \star$ & 22 & $<1.0$ \\
\hline P?US & .0742 & $32 *$ & $<1$ & 9 & 10 & 49 & 4.8 \\
\hline \multicolumn{8}{|l|}{ Elements: } \\
\hline ts & .0122 & $40 \star$ & $<1$ & 18 & $30 *$ & 12 & 2.2 \\
\hline $\mathrm{Ba}$ & .1032 & 6 & $<1$ & 25 & $64 *$ & 3 & $<1.0$ \\
\hline$C, 0 r a$ & .1040 & $19 *$ & 12 & 8 & $57 \star$ & 4 & 1.2 \\
\hline$c, \cos 3$ & .2119 & 4 & $<1$ & 18 & $33 \star$ & 44 & $<1.0$ \\
\hline Co & .1344 & $25 \star$ & $<1$ & 26 & $47 *$ & 3 & 1.0 \\
\hline $\mathrm{Cr}$ & .0172 & $27 \star$ & $<1$ & 22 & $46 *$ & 4 & 1.2 \\
\hline $\mathrm{Cu}$ & .0049 & $27 *$ & 15 & $<1$ & $57 *$ & 1 & 1.3 \\
\hline $\mathrm{Fe}$ & .0516 & $46 *$ & $<1$ & 5 & $14 *$ & 35 & 8.8 \\
\hline$G a$ & $.0<59$ & $<1$ & $<1$ & $38 *$ & $28 *$ & 34 & $<1.0$ \\
\hline $\mathrm{Hg}$ & .3342 & 15 & 4 & $37 *$ & $16 *$ & 28 & $<1.0$ \\
\hline$m n$ & .1365 & $37 \star$ & 5 & 16 & $41 \star$ & 1 & 3.2 \\
\hline MO & .1420 & 22 & $<1$ & $38 *$ & $38 *$ & 2 & $<1.0$ \\
\hline$i j$ & .1349 & $35 *$ & $<1$ & 13 & $51 *$ & 1 & 1.8 \\
\hline$P b$ & .0470 & $20 *$ & $<1$ & 24 & $37 \star$ & 18 & 1.7 \\
\hline Sc & .0032 & 3 & $<1$ & 18 & $4 J *$ & 38 & $<1.0$ \\
\hline$s r$ & .0251 & $12 \star$ & $<1$ & 28 & $48 \star$ & 12 & $<1.0$ \\
\hline v & $.1 C 03$ & 11 & $<1$ & 28 & $58 *$ & 2 & $<1.0$ \\
\hline$Y$ & .0155 & $11 *$ & 8 & 20 & $46 *$ & 16 & $<1.0$ \\
\hline $2 r$ & $.0 C 79$ & $<1$ & 14 & $<1$ & $64 *$ & 22 & $<1.0$ \\
\hline
\end{tabular}

$1 / v(m)$ is slightly biased to the high side because of an imbalance in the sample design due to sample rejection.

21 Computea on untransformed data. 
Table 3.1.--Comonents of yeochemical variance for shale of lower inississippian a se in kentucky. [Components given as percentages of total logarithmic variance. Total $V(\log x)$; $\star$, component significantly different from zero d the li. lis probability level; see text for explanation of $v(m) j$

\begin{tabular}{|c|c|c|c|c|c|}
\hline Total & Bet ween & Between & Between & Eetween & Between \\
\hline$(\log x)$ & $\begin{array}{c}\text { Areas } \\
V(A)\end{array}$ & $\begin{array}{l}\text { Quads } \\
V(: 3)\end{array}$ & $\begin{array}{c}\text { Localities } \\
V(C)\end{array}$ & $\begin{array}{c}\text { Sainples } \\
V(v)\end{array}$ & $\begin{array}{c}\text { Keplicates } \\
V(D)\end{array}$ \\
\hline
\end{tabular}

Oxides:

\begin{tabular}{|c|c|c|c|c|c|c|c|}
\hline 510221 & 67.05 & $40 \% *$ & $<1 \%$ & $<1 \%$ & $60 \% *$ & $<1 \%$ & 5.4 \\
\hline$A 1203$ & .0153 & 7 & 9 & $<1$ & $84 *$ & $<1$ & $<1.0$ \\
\hline te20s & .0370 & $\ddot{\varepsilon}$ & $<1$ & 27 & out & 5 & $<1.0$ \\
\hline $\mathrm{FeO}$ & .0648 & $42 \star$ & 2 & $<1$ & $49 *$ & $\ddot{\varepsilon}$ & 5.7 \\
\hline$\because 40$ & .0601 & $43 \star$ & 4 & $<1$ & $48 *$ & 1 & 4.1 \\
\hline$C a O$ & .5118 & $18 \star$ & $2.7 \star$ & $<1$ & $44 *$ & 12 & $<1.0$ \\
\hline iva? & .0841 & 5 & $22 *$ & $<1$ & $1 u$ & 03 & $<1.0$ \\
\hline$k \geq 0$ & .0192 & $23 \star$ & $1 \varepsilon$ & $<1$ & $52 *$ & 7 & 1.5 \\
\hline $1420+$ & .0144 & $<1$ & $<1$ & $<1$ & $60 *$ & 34 & $<1.0$ \\
\hline Tiv? & .0123 & 14 & $42 \star$ & $<1$ & $42 *$ & 2 & $<1.0$ \\
\hline$P 2 \cup 5$ & .1397 & $<1$ & $42 *$ & $<1$ & 12 & 45 & $<1.0$ \\
\hline
\end{tabular}

Elements:

\begin{tabular}{|c|c|c|c|c|c|c|c|}
\hline ij & .0327 & $18 *$ & $15 *$ & $<1$ & 01 1* & 6 & 1.2 \\
\hline ua & .0212 & 2 & $<1$ & $<1$ & $71 \star$ & 0 & $<1.0$ \\
\hline $\mathrm{CO}$ & .0389 & $24 \star$ & $<1$ & 10 & $49 \star$ & 17 & 2.5 \\
\hline$c r$ & .0140 & 1 & $<1$ & $<1$ & $86 \star$ & 11 & $<1.0$ \\
\hline $\mathrm{Cu}$ & .0885 & 4 & $<1$ & 16 & $69 \star$ & 7 & $<1.0$ \\
\hline inn & .0554 & 7 & 5 & $<1$ & $80 *$ & 3 & $<1.0$ \\
\hline is $i$ & .0661 & 43 & $20 \star$ & 3 & $32 \star$ & 2 & 2.9 \\
\hline sc & .0000 & 19 & $<1$ & 5 & $60 \star$ & 15 & 2.0 \\
\hline Sr & .01604 & $20 \star$ & 7 & $<1$ & $5 i \star$ & 21 & 1.7 \\
\hline$v$ & .0241 & $<1$ & 12 & $<1$ & 81 ¿ & 7 & $<1.0$ \\
\hline Y & .15282 & 12 & $<1$ & $44 \star$ & $35 \star$ & 9 & $<1.0$ \\
\hline$\angle r$ & .0817 & $71 \star$ & $13 \star$ & $<1$ & $13 *$ & 2 & 0.6 \\
\hline
\end{tabular}

$1 / v(m)$ is slightly oiased to the nigh side because of an imbalance in the sample design due to sample rejection.

2! concuted on untransformed data. 
Table 5C.--Components of geochemical variance for shale of Upper Mississippian age in kentucky. [Components given as percentages of total logarithmic variance. Total $V(\log x) ; \quad \star$, component significantly different from zero at the 0.05 probability level; see text for explanation of $v(m)]$

Oxides:

$\begin{array}{cccccc}\text { Total } & \text { Between } & \text { Between } & \text { Between } & \text { Between Between } & (m) 1 / \\ V(\log x) & \text { Areas } & \text { Quads } & \text { Localities Samples Replicates } & \\ & V(A) & V(B) & V(C) & V(D) & V(O)\end{array}$

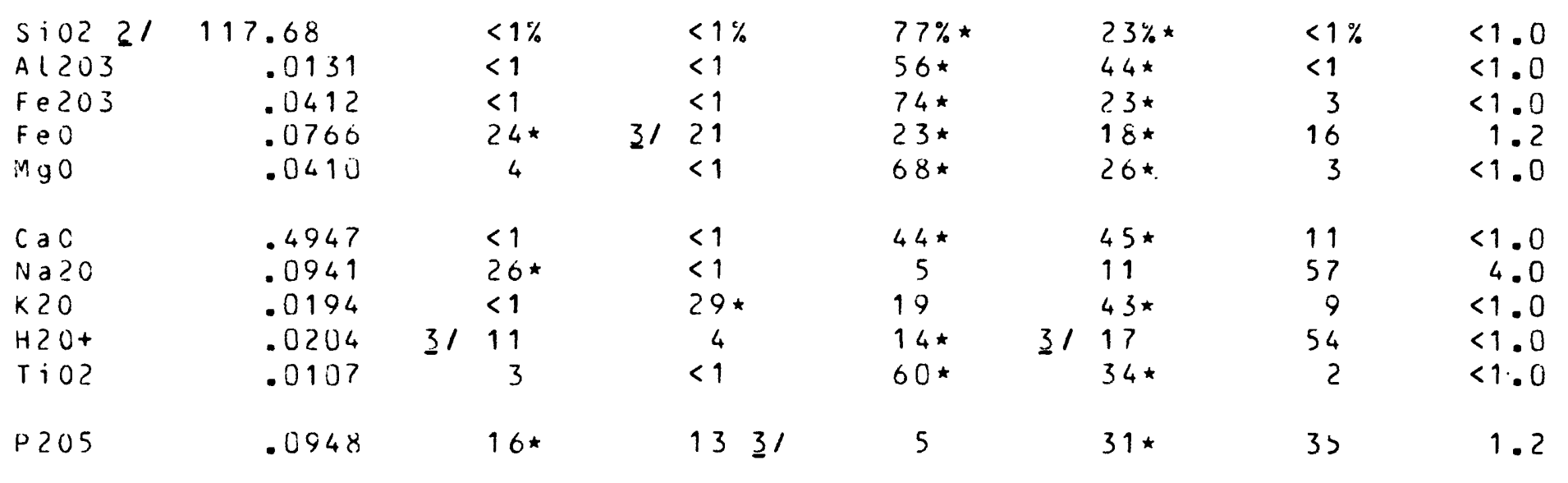

Elements:

\begin{tabular}{|c|c|c|c|c|c|c|c|}
\hline B & .0412 & $<1$ & 23 & $47 \star$ & $20 *$ & 11 & $<1.0$ \\
\hline$B a$ & .0217 & $39 \star$ & $<1$ & 17 & $34 \star$ & 11 & 4.3 \\
\hline $\mathrm{CO}$ & .0353 & 2 & $27 \star$ & $<1$ & $54 *$ & 16 & $<1.0$ \\
\hline $\mathrm{Cr}$ & .0115 & $29 *$ & $<1$ & 2 & $44 *$ & 24 & 3.9 \\
\hline $\mathrm{Cu}$ & .0929 & $<1$ & 15 & $34 \star$ & $45 \star$ & 6 & $<1.0$ \\
\hline $\mathrm{Ga}$ & .0248 & $<1$ & 1 & $63 *$ & $20 \star$ & 16 & $<1.0$ \\
\hline$m n$ & .2204 & 16 & $<1$ & 49 * & $32 \star$ & 3 & $<1.0$ \\
\hline Ni & .0218 & 13 & $<1$ & $54 \star$ & $25 *$ & 8 & $<1.0$ \\
\hline SC & .0076 & 8 & $<1$ & $51 \star$ & $17 \star$ & 25 & $<1.0$ \\
\hline Sr & .0769 & $3 / 19$ & $<1$ & $65 *$ & $3 / 4$ & 12 & 1.1 \\
\hline v & .0184 & $3 / 10$ & $<1$ & $3 / 31$ & $38 *$ & 22 & $<1.0$ \\
\hline Y & .0161 & $<1$ & 3 & $3 / 36$ & $44 \star$ & 17 & $<1.0$ \\
\hline $2 r$ & .0451 & 1 & 6 & $46 *$ & $37 *$ & 10 & $<1.0$ \\
\hline
\end{tabular}


Tajle 50.-- components of yeochemical variance for shale of pennsylvanian aye in kentucky. [Components given as percentages of total lojarithmic variance. rotal $v(\log x)$; * component significantly different from zero at the 0 . ns provability level; see text for explandtion of $v(r)$ ]

$0 \times$ iues:

\begin{tabular}{|c|c|c|c|c|c|}
\hline Total & Between & between & detween & Between & Between \\
\hline$v\left(\begin{array}{ll}\log x & x\end{array}\right)$ & $\begin{array}{l}\text { Areas } \\
v(A)\end{array}$ & $\begin{array}{l}\text { auds } \\
v(B)\end{array}$ & $\begin{array}{l}\text { Localities } \\
V(C)\end{array}$ & $\begin{array}{l}\text { Samples } \\
V(0)\end{array}$ & $\begin{array}{c}\text { Renlicates } \\
V(D)\end{array}$ \\
\hline
\end{tabular}

\begin{tabular}{|c|c|c|c|c|c|c|c|}
\hline SiOz $\geq 1$ & 08.91 & $<1 \%$ & $6 \%$ & $24 \%$ & $63 \%$ & $2 \%$ & $<1.0$ \\
\hline$A \backslash 203^{\circ}$ & . (1) 151 & 3 & $<1$ & $3 / 29$ & $5 u^{\circ}$ & 18 & $<1.0$ \\
\hline $\mathrm{Fe} 203$ & .0602 & 5 & $<1$ & $33 *$ & $58 *$ & 4 & $<1.0$ \\
\hline $\mathrm{FeO}$ & .1513 & 11 & $22 \star$ & 3 & $47 \star$ & 17 & $<1.0$ \\
\hline$\therefore$ gau & .17710 & $<1$ & $28 \star$ & 7 & $59 *$ & 0 & $<1.0$ \\
\hline $\mathrm{CaU}$ & .1061 & $10 *$ & $<1$ & 1 & 10 & 72 & 1.5 \\
\hline NalO & .1206 & $11 \star$ & $3 / 15$ & 2 & $20 *$ & 46 & $<1.0$ \\
\hline 1220 & .0408 & $<1$ & $<1$ & $54 *$ & $40 *$ & 0 & $<1.0$ \\
\hline $1+20+$ & .0236 & 1 & 2 & $36 *$ & $55 *$ & 7 & $<1.0$ \\
\hline Tio? & .0082 & $<1$ & 12 & $<1$ & $8<\star$ & 7 & $<1.0$ \\
\hline 0205 & $.111 u$ & $<1$ & 14 & $<1$ & $53 *$ & 33 & $<1 . C$ \\
\hline
\end{tabular}

Elements:

\begin{tabular}{|c|c|c|c|c|c|c|c|}
\hline :3 & $.024 t$ & $34 *$ & 4 & $<1$ & $4 u *$ & 22 & 4.2 \\
\hline tja & .03 \% & $<1$ & $26 *$ & 13 & $50 *$ & 5 & $<1.0$ \\
\hline $\mathrm{CO}$ & .1314 & $14 \star$ & $<1$ & 14 & $27 \star$ & 12 & 1.2 \\
\hline $\mathrm{Cr}$ &.$\cup 170$ & 0 & $<1$ & 10 & $50 *$ & 20 & $<1.0$ \\
\hline $\mathrm{Cu}$ & .0854 & $<1$ & 11 & 22 & $58 *$ & $\ddot{c}$ & $<1.0$ \\
\hline Ga & $.0) 411$ & $<1$ & 7 & 13 & $75 *$ & 5 & $<1.0$ \\
\hline in & .2357 & 12 & $<1$ & $51 *$ & $30 *$ & 7 & $<1.0$ \\
\hline $\mathrm{Ni}$ & .01000 & $3 / 14$ & 7 & $40 *$ & $33 \star$ & 7 & $<1.0$ \\
\hline$P 0$ & .10359 & $15 *$ & $<1$ & 8 & $24 \star$ & 53 & 1.8 \\
\hline$S c$ &. $\operatorname{CLC} 3$ & $<1$ & $<1$ & $53 *$ & $30 *$ & 17 & $<1.0$ \\
\hline$j r$ & .0553 & $<1$ & 11 & $26 *$ & $49 *$ & 12 & $<1.0$ \\
\hline v & .0378 & $<1$ & $<1$ & $3 / 28$ & $07 *$ & 5 & $<1.0$ \\
\hline Y & .0404 & $<1$ & 18 & $<1$ & $74 *$ & 9 & $<1.0$ \\
\hline $2 r$ & .3470 & $<1$ & $28 *$ & 17 & $48 *$ & 7 & $<1 . C$ \\
\hline
\end{tabular}

1/ v(r) is slightly biased to the high side decause of an imalance in the sample cesiyn due to sample rejection.

?. Conuuted on untransforined data.

$3 /$ Significant at the 0.1 frobability level. 
Table OA.--Summary geochemical statistics for the organic-rich Chat tanooga, New Albany, and Onio Shales in kentucky. [Data are in parts per million except where noted as percent (\%); GM, geometric mean; GD. geometric deviation; GE, geometric error; Ratio, number of analyses in which constituent was determined to total number of analyses; leaders (--) indicate insufficient dat a ]

Part I. Statewide summary

\begin{tabular}{|c|c|c|c|c|}
\hline Oxides: & GM & GD & $G E$ & Ratio \\
\hline $\begin{array}{l}\text { SiOU2, } \% \text { 1/ } \\
\text { Al } 203, \% \\
\text { ingo, \% } \\
\text { CaO \% \% } \\
\text { Na20, \% }\end{array}$ & $\begin{array}{l}25.0 \\
13 \\
1.1 \\
.28 \\
.35\end{array}$ & $\begin{array}{l}6.2 \\
1.13 \\
1.45 \\
2.84 \\
1.74\end{array}$ & $\begin{array}{l}<0.6 \\
1.01 \\
1.12 \\
1.71 \\
1.49\end{array}$ & $\begin{array}{l}88: 88 \\
88: 88 \\
88: 88 \\
82: 88 \\
80: 88\end{array}$ \\
\hline $\begin{array}{l}\text { K20, \% } \\
\text { H2O+, \% } \\
\text { TiO2, \% } \\
\text { P } 205,\end{array}$ & $\begin{array}{c}3.6 \\
4.8 \\
.76\end{array}$ & $\begin{array}{l}1.16 \\
1.46 \\
1.15 \\
1.81\end{array}$ & $\begin{array}{l}1.08 \\
1.41 \\
1.07 \\
1.55\end{array}$ & $\begin{array}{l}88: 88 \\
88: 88 \\
88: 88 \\
80: 88\end{array}$ \\
\hline
\end{tabular}

Elements:

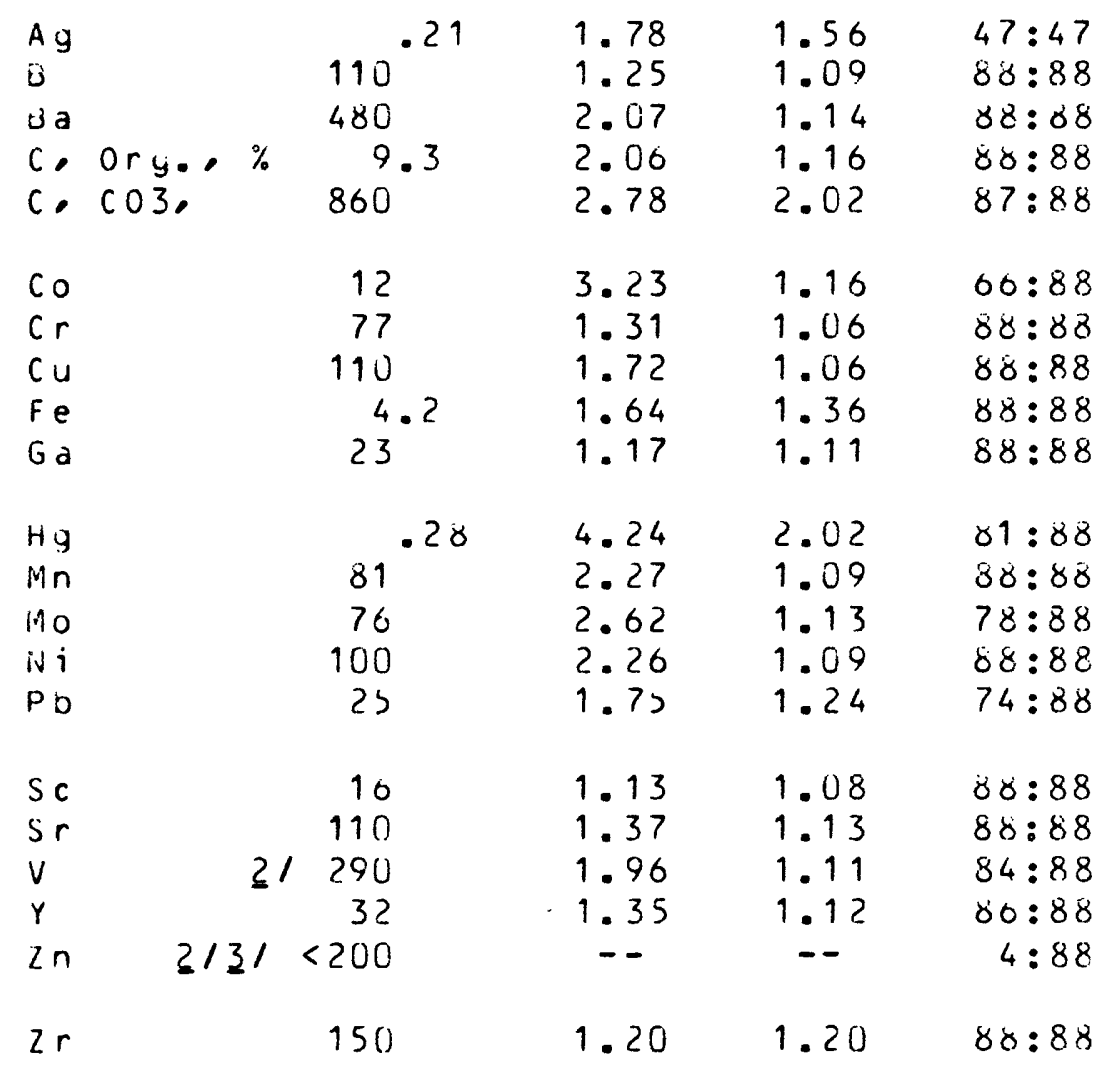


Table OA.-- cont.

Part II. piedians oy areas (quadrangle pairs)

[rhe number of saniwles in each area is given in parentheses]

Eriensoury-Petroleum- ureeding- Knifely- Eli-

Eddyville Austin Eurksville

(8)

(16)

(16)

ounnville

(16)

roahead

Manchester

I slands-

(16) Burtonville

(16)

60

Cave \% $2.7 \%$

P205. $1.6 \%$

E 1.22

C. ur.. \% 1.9,

Co $2 \cdot \mathrm{Us}$

1.29

1.05

1.47

1.90

1.98

0.15

.07

180

2.3

$<\varepsilon$

0.47

.14

110
9.7
17

0.30

.10

100

13

28

90

33

2.8

94

190

44

52

4.0

160

220
05
110
2.9
08
140

1. 20

.11

110

12

10

70

115

4.2

82

200

29

28

0.30
.13

$1 \mathrm{CO}$

12

32

$t c$

96

2.9

76

100
0.11
.055

1.50

33

29

30

110

9.7

$<9$

1. 41

120

90

37

110

33

190
84

120

2.3

44

05

21

110

110

$1 /$ iomolded on untransformed ata.

I' Erickson (1000) reports up to 1000 oarts per million each of $v$ and In in the sunoury shale (an organic rich inississiopian shale similar to the Chat anooya and lying aoove $i t$ ) in the friendsnip quadrangle of northeastern kentucky.

$3 / 923 \mathrm{Npm} Z \mathrm{n}$ ireasured in samule USH-K122. (Cnattanooya Snale, knifely cisaurangle): 1000 pim $z$ n measured in sample OSH-R121 (Chat tanooga shale. Petroleum quadrangle). 
Table ob.--summary statistics for shale, siltstone, and sandy shale of Lower inississippian age in kentucky. [Data are in parts per million except where noted as percent (\%): GM, geometric mean; GD, geometric aeviation: GE. geometric error: Me median: Ratio, number of analyses in which constituent was determined to total number of analyses: leaders (--) indicate insufficient data]

Part I. Statewide summary

\section{Shale and siltstone $1 /$}

$0 \times$ ides:$$
\text { Al } 203, \% \text { ? I } 69
$$

$\mathrm{Feu}$. \%

$\mathrm{MgO}$, \%

$\mathrm{CaO}, \%$

Na20, \%

$\mathrm{K} 2 \mathrm{U}, \%$

$\mathrm{H} 2 \mathrm{OH}, \%$

Ti02, \%

P 205 .

$\mathrm{CO} 2 \%$
GM GD GE Ratio

69

12

\section{7}

1.1

1.5

7.4

0.4

1.30

1.02

1.54

1.11

$1.74 \quad 1.17$

$1.67 \quad 1.07$

$60: 60$

$60: 60$

$60: 60$

$60: 60$

$60: 60$

$$
.65
$$

.53

$$
4.37
$$

1.76

1.70

1.09

$$
2.6
$$

2.7

.73

1.83

1.35

1.28

1.18

1.04

$58: 60$

$60: 60$

$60: 60$

$60: 60$

$60: 60$

1.28

570

31.02

2.07

1.78

3.4

$58: 60$

$31: 60$
Sandy Shale

M Ratio

$57 \quad 12: 12$

$6.0 \quad 12: 12$

$1.8 \quad 12: 12$

$.69 \quad 12: 12$

$4.1 \quad 12: 12$

$12 \quad 12: 12$

$.60 \quad 12: 12$

$1.2 \quad 12: 12$

$1.5 \quad 12: 12$

$.3612: 12$

$700 \quad 12: 12$

\begin{tabular}{|c|c|c|c|c|c|c|}
\hline$B$ & 92 & 1.37 & 1.11 & $59: 60$ & $<30$ & $0: 12$ \\
\hline $8 a$ & 370 & 1.35 & 1.09 & $60: 60$ & 150 & $12: 12$ \\
\hline Co & 11 & 1.49 & 1.20 & $50: 60$ & $<8$ & $6: 12$ \\
\hline $\mathrm{Cr}$ & 71 & 1.28 & 1.09 & $60: 6 C$ & 43 & $12: 12$ \\
\hline $\mathrm{Cu}$ & 17 & 1.84 & 1.20 & $59: 60$ & 9 & $10: 12$ \\
\hline Ga & 18 & 1.45 & 1.11 & $54: 60$ & 10 & $7: 12$ \\
\hline La & 46 & 1.33 & 1.13 & $4: 60$ & $<70$ & $0: 12$ \\
\hline inn & 190 & 1.62 & 1.10 & $60: 60$ & 120 & $12: 12$ \\
\hline No & $<20$ & -- & -- & $0: 60$ & $<20$ & $0: 12$ \\
\hline $\mathrm{Ni}$ & $41 \quad 49$ & 1.75 & 1.09 & $60: 60$ & 20 & $12: 12$ \\
\hline $\mathrm{Pb}$ & 19 & 1.35 & 1.28 & $28: 60$ & $<20$ & $2: 12$ \\
\hline S c & 16 & 1.19 & 1.08 & $60: 60$ & 12 & $11: 12$ \\
\hline$S r$ & 86 & 1.58 & 1.31 & $57: 60$ & 110 & $12: 12$ \\
\hline$v$ & 130 & 1.37 & 1.10 & $60: 60$ & 57 & $12: 12$ \\
\hline$Y$ & 32 & 1.36 & 1.12 & $58: 60$ & 22 & $9: 12$ \\
\hline $2 n$ & 300 & 1.42 & 1.45 & $4: 60$ & $<500$ & $0: 12$ \\
\hline $2 r$ & 180 & 1.81 & 1.10 & $60: 60$ & 78 & $12: 12$ \\
\hline
\end{tabular}

$13 \quad 12: 12$

Elements: 
Taole od.--cont.

Part II. liedians by areas (quadrangle pairs)

[The number of samples in eacn area is given in parentheses]

Shale+siltstone:

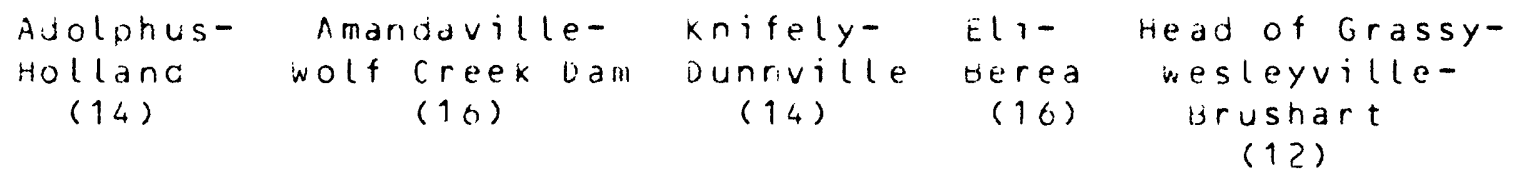

u

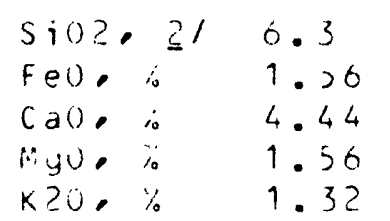

$\begin{array}{ll}67 & 02 \\ .81 & 1.0 \\ .55 & 7.1 \\ 1.7 & 2.3 \\ 3.4 & 2.2\end{array}$
68
.90
.50
1.8
2. 5
70
1.8
.40
1.7
3.1
75
.81
.45
.75
2.0

\begin{tabular}{|c|c|c|c|c|c|c|}
\hline t & 1.40 & $1 \mathrm{w}$ & 74 & 100 & $11:$ & 82 \\
\hline co & 1.49 & 14 & $<8$ & 11 & 12 & 10 \\
\hline Sr & 1.70 & 30 & 140 & 90 & 4L: & 85 \\
\hline $2 r$ & 1.43 & 120 & 85 & 140 & 130 & 490 \\
\hline
\end{tabular}

$1 /$ samkles R11? ana K22? (Adolphus and Dunnville quaurangles. respectively) were excluded from the summary because they are normative carbonates.

2l Computed on untransformed cata.

$3 /$ Primary mode: the distribution is bimodal with a seconoury mode at $5 \%$.

4l sanules 2211 . R212, and $R 221$ (Holland quadrangle) rangeu from $100-270$ ppin vi. 


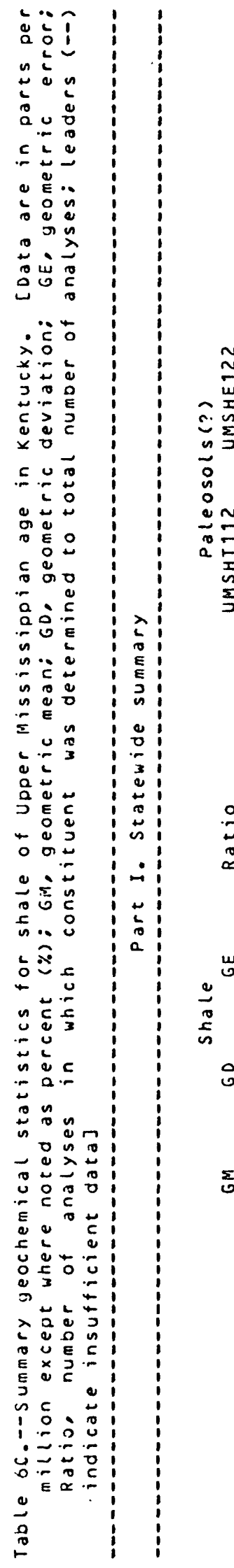

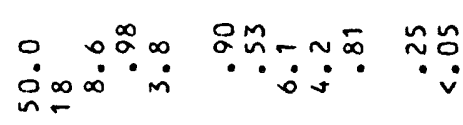

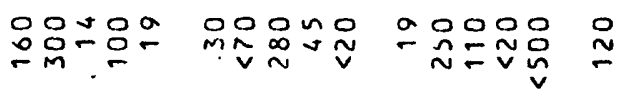

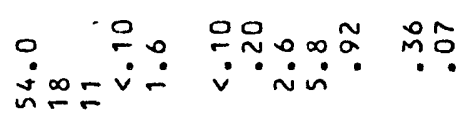

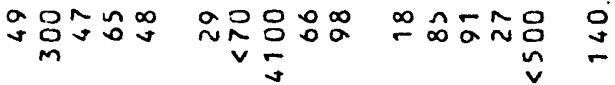

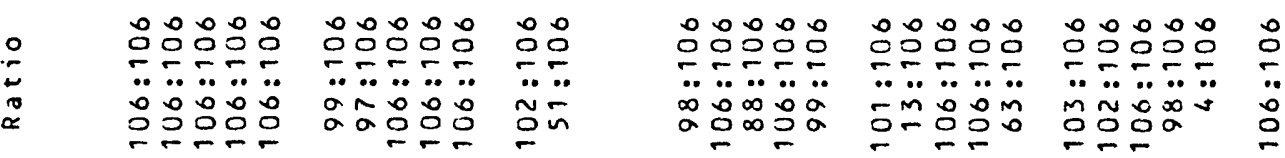

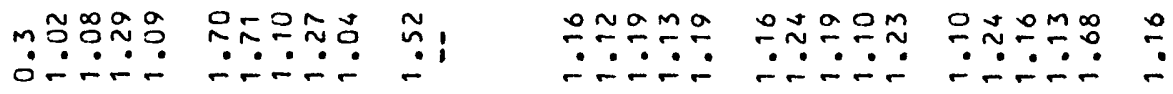

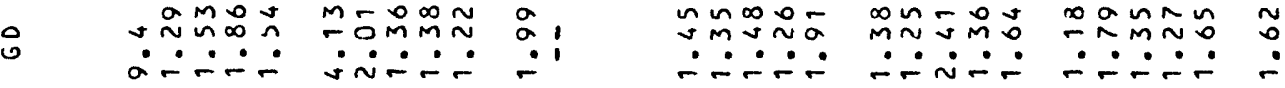

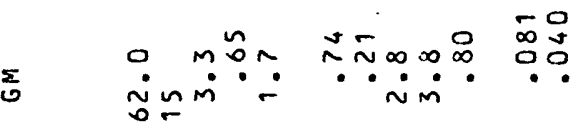

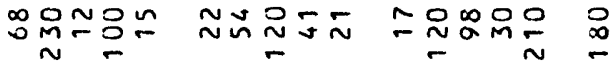

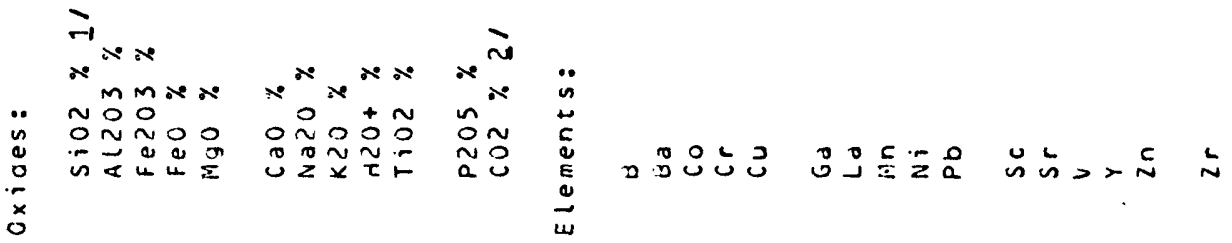




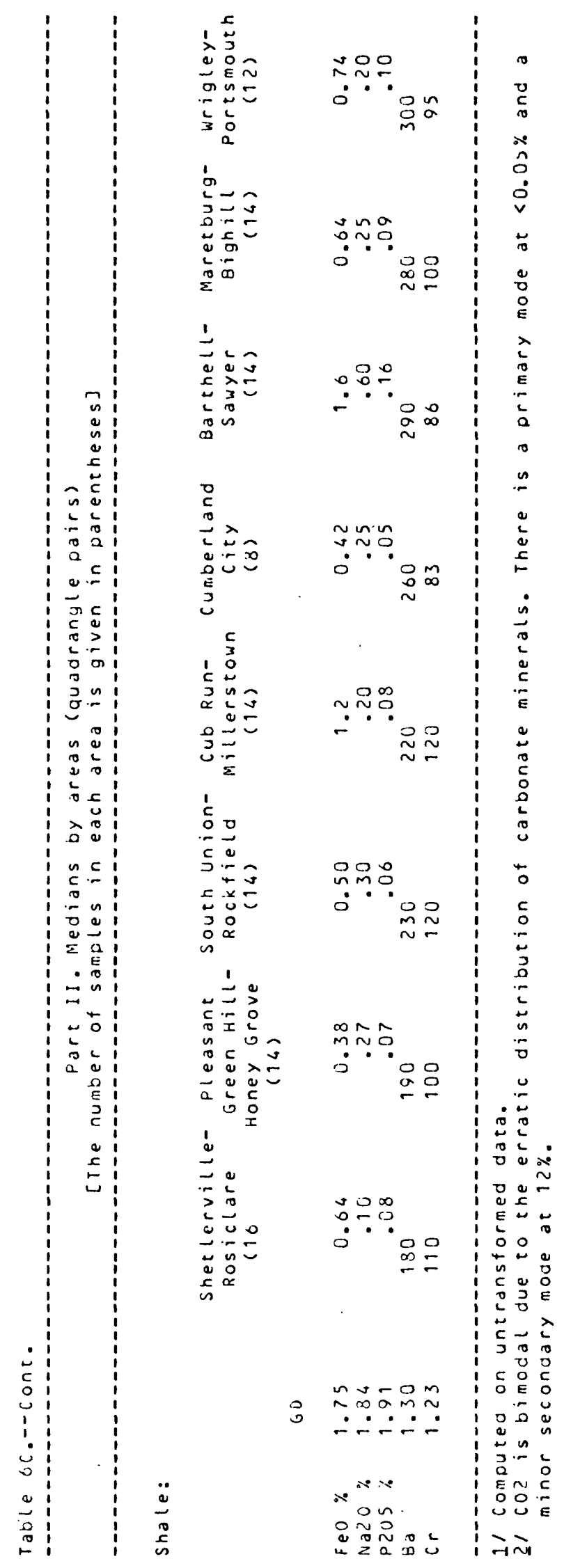




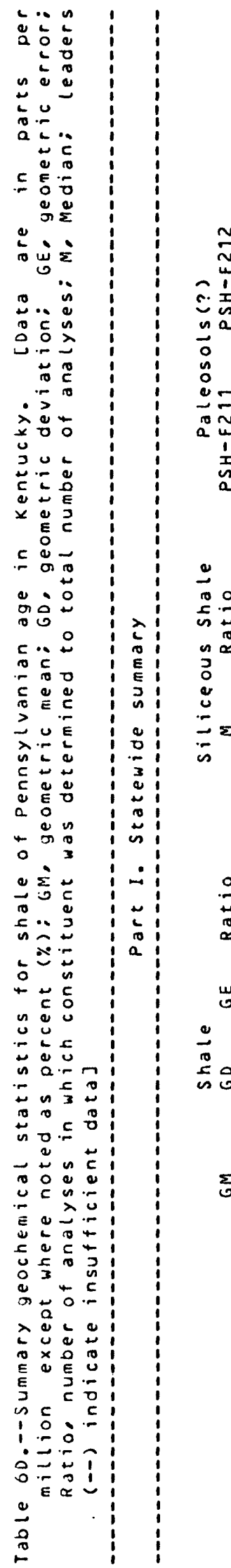

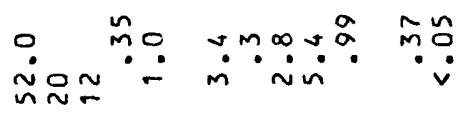

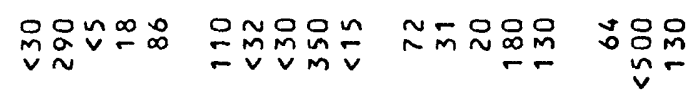

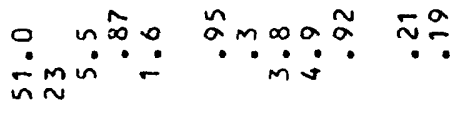

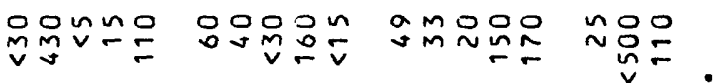

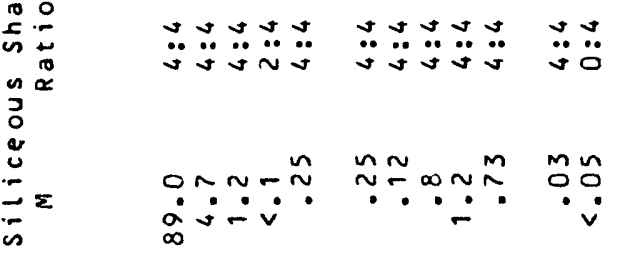

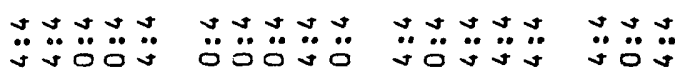

ㅇํำ

0
0
0
0

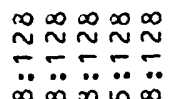

$\infty \infty$

\section{$\infty \infty$}

$\stackrel{\infty}{\sim} \stackrel{\infty}{\sim} \stackrel{\infty}{\sim} \stackrel{\infty}{\sim}$

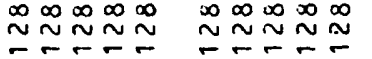

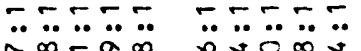

$\sim \stackrel{\infty}{\sim}-\infty \stackrel{\infty}{\sim} \sim \sim \sim 0) \sim$

$\sim \sim \sim \sim \sim$

$\div \div \div \ldots \div$ $\sim \sim \infty \sim \infty \sim \infty$

$\stackrel{\infty}{\sim} m$

mm $⿻$

- $-\tau$

a N $0 \circ$

$\therefore \div-\div \div$

เกษาำ

in

$\sim$

$\infty-\infty n$

$\simeq \approx \sim \simeq$

$\because \because ! m \div$

- IñN

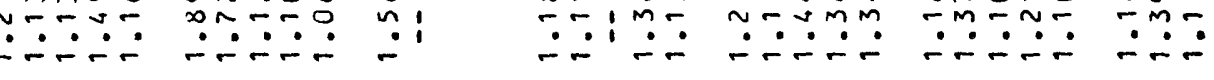

$\stackrel{\infty}{\sim} \stackrel{\infty}{\sim} \stackrel{\infty}{\sim} \stackrel{\infty}{\sim}$

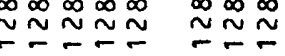

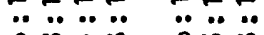

$\approx 0 \stackrel{\infty}{\sim} \cong \stackrel{\infty}{\sim} \stackrel{\infty}{\sim}$

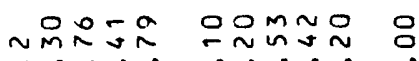

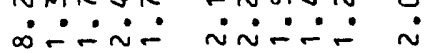

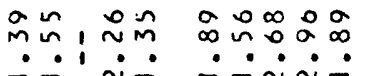

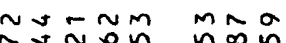

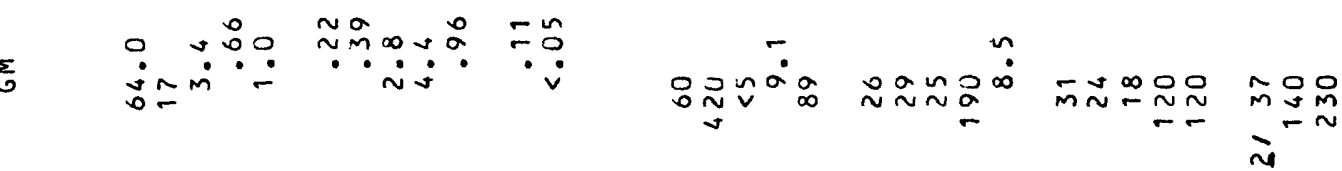

नix

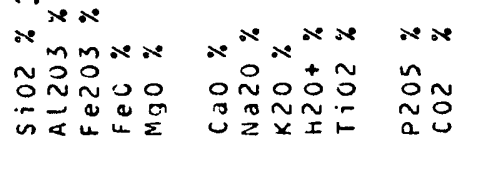

จ 


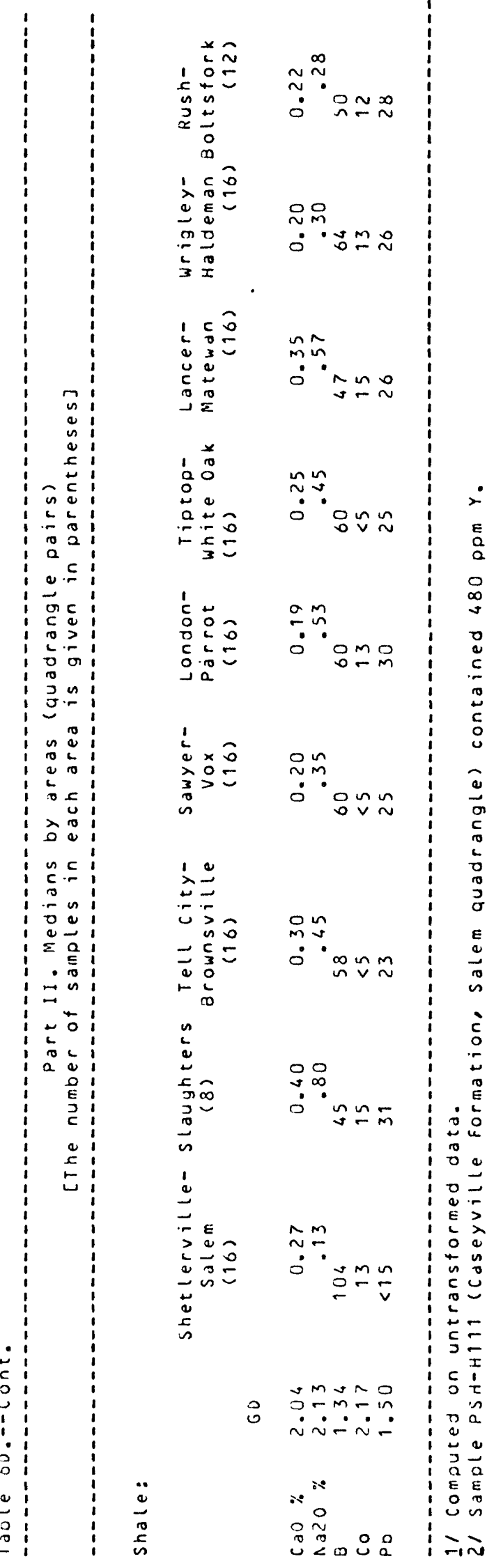

